\title{
Lernen und das Andere
}

Angelika Thielsch 


\title{
Lernen und das Andere
}

\section{Hochschuldidaktische Erkenntnisse zur Anwendung des Konzeptes der Alterität auf Lehr- und Lernprozesse im akademischen Kontext}

\author{
Dissertation \\ zur Erlangung des Doktorgrades \\ der Sozialwissenschaftlichen Fakultät \\ der Georg-August-Universität Göttingen
}

vorgelegt von

Angelika Thielsch

geboren in Kassel

Göttingen, 2019 


\section{Mitglieder des Thesis Committee}

Prof. Dr. Ariane S. Willems Erstbetreuerin \& Gutachterin

Georg-August-Universität Göttingen

Dr. Andreas Fleischmann

Zweitbetreuer \& Gutachter TU München

Prof. Dr. Antonia Scholkmann Drittbetreuerin Aalborg University

Tag der mündlichen Prüfung

7. Juni 2019 
Meine Begeisterung im Begegnen des Anderen wurde begleitet und getragen durch

Offenheit und Wertschätzung, Weitblick und Innehalten,

Geduld und Fröhlichkeit vieler wunderbarer Menschen.

\section{Dafür ein herzlicher Dank!}

An die inspirierende Gruppe, die ich mein Thesis Committee nennen durfte: meine Doktormutter Prof. Dr. Ariane S. Willems, mein Zweitbetreuer Dr. Andreas Fleischmann, meine Drittbetreuerin Prof. Dr. Antonia Scholkmann.

An das tollste Team, meine denk- und backfreudige, herzlich-konstruktive Arbeitsfamilie der Göttinger Hochschuldidaktik, an famose Kolleg*innen aus benachbarten Teams und meiner grandiosen Fachcommunity.

An die Menschen, die meinem Herz die wichtigsten sind, die mein Ich trotz und auch wegen meiner Freude am Tun in diesen drei Jahren nie aus den Augen verloren, die mir den Blick zurück auf mich erleichterten, wann immer die Begegnung mit dem Anderen mich mal rüttelte und schüttelte, die nie müde werden wollten, mir die eigene Begeisterung zu spiegeln.

Euch hab` ich lieb! 



\section{Inhaltsverzeichnis}

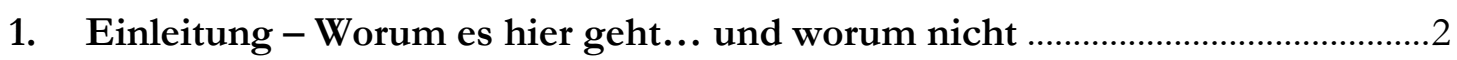

2. Theorie \& Erkenntnisinteresse - Wieso das wichtig ist..................................... 5

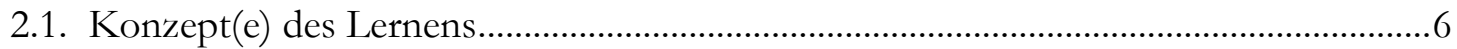

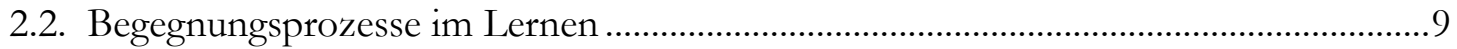

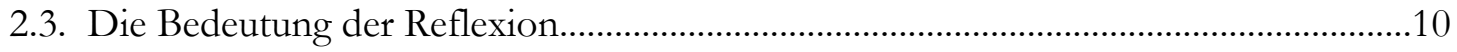

2.4. Das Ich im (akademischen) Lernen ..............................................................................13

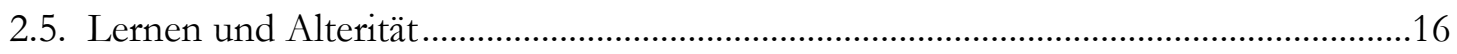

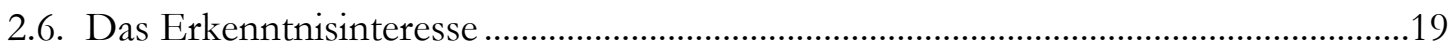

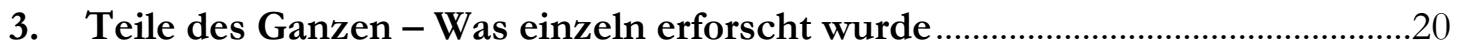

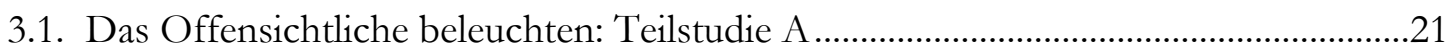

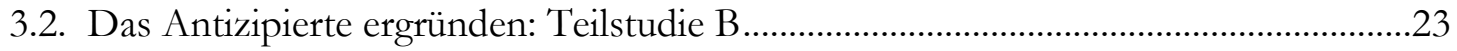

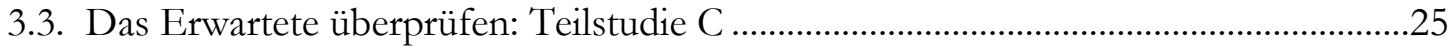

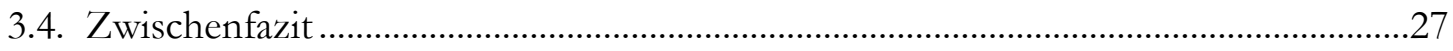

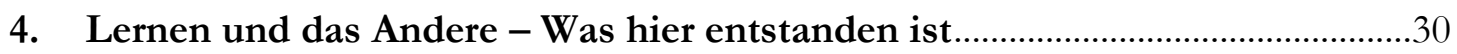

4.1. Alterität als Instrument für die Bildungswissenschaften.................................................30

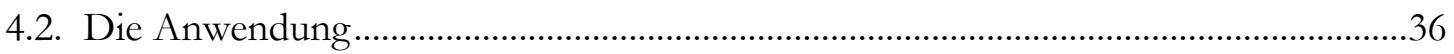

4.2.1. Teilstudie A: Verborgene Erwartungen \& akademische Sozialisation ...................36

4.2.2. Teilstudie B: Akademisches Selbst \& kollektives Infragestellen...............................38

4.2.3. Teilstudie C: Gegenseitiges Begründen \& individuelles Erkennen .........................39

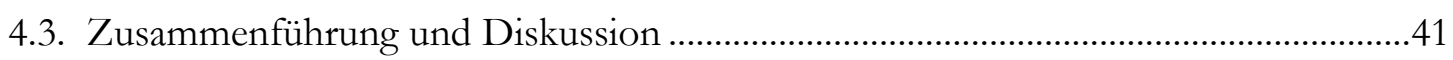

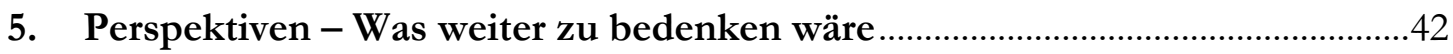

6. Reflexion - Wo ich dem Anderen begegnet bin ...................................................4

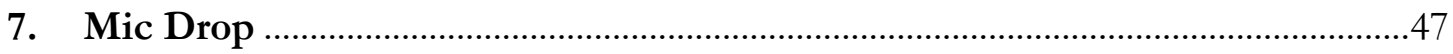

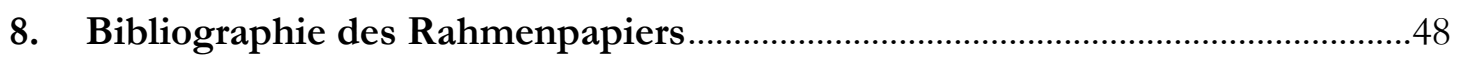

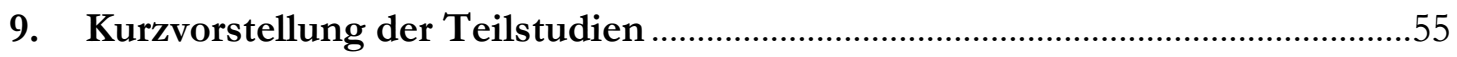

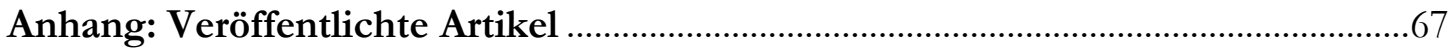




\title{
1. Einleitung - Worum es hier geht... und worum nicht
}

\author{
„,[D]er bildende Diskurs soll nichtnormal sein, uns durch die Kraft \\ seiner Fremdartigkeit aus unserem alten Selbst herausführen, \\ dazu beitragen, daß wir andere Wesen werden"
}

(Rorty, 2017[1987], S. 390).

Lernen erfolgt eingebettet in einen sozialen Kontext. Es ist dieser Kontext, der mit seinen Rahmenbedingungen und Sinnstrukturen Einfluss darauf nimmt, was und wie eine Person lernt und welche Erfahrung sie für ihren Lernprozess als bedeutsam erachtet. ${ }^{1}$ Die Relevanz von Erfahrungen für das Lernen - sowie Bildungsprozesse generell - ist unbestritten ${ }^{2}$, ebenso die Notwendigkeit der Reflexion ${ }^{3}$. In Verbindung zu und Aushandlung mit den korrespondierenden abstrakten Informationen stellen sie die drei Kernelemente jener Prozesse dar, durch die eine Person neues Wissen erwirbt.

In meiner Arbeit nutze ich dieses Grundverständnis menschlichen Lernens und stelle einen weiteren Zugang zum Verstehen und Fördern derlei lernrelevanter Reflexions- und Erfahrungsprozesse für den akademischen Kontext vor. Unter Verwendung des Konzeptpaares der Identität und Alterität ${ }^{4}$ argumentiere ich, inwiefern es die Begegnung mit dem Anderen ist, die Anlass zur Reflexion gibt, und dass derlei Reflexion in ihrer Bedeutung für das Lernen aus mehreren alteritätsbezogenen Perspektiven ergründet werden kann: Aus Perspektive des Andersseins der Anderen, des Andersseins des Themas und des Andersseins des Systems. In Relation zu allen steht das in der Begegnung evozierte Anderssein des Ich. In meiner Dissertation habe ich das lernförderliche Potential von Begegnungsprozessen mithilfe des Konzeptes der Alterität erforscht und so dieses Phänomen, das ein Mensch im (Re)Konstruieren der eigenen Identität erfährt, als analytischen Zugang für bildungswissenschaftliche Kontexte begründet. Die Ergebnisse ebenso wie die sich hieran anschließenden Überlegungen sind Gegenstand dieses Rahmenpapiers.

In Kapitel 2 werden zunächst die theoretischen Hintergründe skizziert, die für mein Forschungshandeln leitend waren. So werden die Relevanz der Reflexion für Lernprozesse, die Dimensionen der Begegnung sowie die damit verbundene Existenz des Anderen im Lernen erörtert, nachdem einleitend das hier verwendete Verständnis der Prozesselemente im Lernen vorgestellt wurde. Anschließend skizziere ich Erkenntnisse zur Konstruktion individueller Identitäten in Relation zum wahrgenommenen Anderen, verdeutliche den Einfluss der Sozialisation hierfür und leite davon grundlegende Annahmen zur Entstehung lernförderlicher Reflexionsimpulse ab. Basierend darauf formuliere ich zum Abschluss dieses

${ }^{1}$ Vgl. u.a. Boud \& Walker (1990), Künkler (2011), Ramsden (1997), Wenger (1998).

2 Vgl. u.a. Faulstich \& Bracker (2015), Kolb (1984), Meyer-Drawe (2003), Mezirow (2000).

3 Vgl. u.a. Dewey (1910), Fink (2013), Schön (1983 \& 1987).

${ }^{4}$ Alterität kann definiert werden als „[...] jene Phänomene, in denen ein Selbst seinem Außen begegnet und es als Anderes konstruiert, um sich der eigenen Identität zu vergewissern“ (Thielsch, 2019c i. E.). 
Kapitels das Erkenntnisinteresse meines Handelns. Als übergeordnetes Ziel meiner Arbeit wird hier expliziert, ein alteritätsbezogenes Lernverständnis zu begründen, das bestehende subjektbezogen-genealogische Theorien des Lernens um eine weitere Facette zu ergänzen vermag. Um dies zu erreichen - so kündige ich an dieser Stelle an - wurde das hier elaborierte Alteritätsverständnis zunächst forschend auf Situationen des Lernens appliziert und anschließend sein analytisches Potential als Instrument im Ergründen akademischer LehrLernprozesse systematisiert.

Ausgehend von diesen Begründungslinien ermöglicht Kapitel 3 einen Einblick in die einzelnen Forschungsfelder und -designs des gesamten Dissertationsprojektes und bildet so die kontextuelle und methodologische Rahmung der Arbeit als Ganzes. Die drei aufeinander aufbauend geplanten und methodisch unterschiedlich ausgestalteten Teilstudien meiner Forschung werden sodann mit Blick auf die Pluralität von Begegnungsprozessen mit dem Anderen umrissen und die jeweiligen Haupterkenntnisse für die übergeordnete Forschungsfrage herausgearbeitet.

Kern dieses Rahmenpapiers und Höhepunkt des gesamten Dissertationsprojektes stellt das vierte Kapitel dar, in dem basierend auf den Erkenntnissen der Einzelstudien die in ihnen ermittelten Begegnungsprozesse mit dem Anderen analysiert werden. Dies einleitend stelle ich hier jenes Modell vor, das nunmehr als Instrument genutzt werden kann, um akademische Lehr- und Lernkontexte aus Perspektive der Alterität zu hinterfragen, die darin enthaltenen Reflexionsimpulse zu ermitteln und zu gestalten. In diesem Kapitel wird das hier begründete alteritätsbezogene Lernverständnis expliziert und sein Potential für die weitere Anwendung in Bildungskontexten nachgezeichnet. Den Abschluss dieses Teils bilden die Zusammenführung und kritische Diskussion der elaborierten Erkenntnisse. Ergänzend bietet Kapitel 5 Gedanken dazu, welche Anknüpfungspunkte sich basierend auf meiner Arbeit für zukünftige Forschungsprojekte ergeben.

Somit bildet Kapitel 2 die theoretische Herleitung der hier realisierten Forschung(en), während Kapitel 3, 4 und 5 ihre Zusammenführung und Auswertung darstellen. Gemeinsam bilden diese Kapitel den erkenntnisbezogenen Kern meiner Dissertation. Ergänzt werden sie in diesem Rahmenpapier durch zwei den Gesamtprozess abschließende Kapitel, die zusammen mit dieser Einleitung - dazu dienen, das Vorgehen und die Erkenntnisse im Zuge der Forschung auf einer übergeordneten Ebene zu thematisieren. Eine Arbeit, die sich mit lernförderlichen Aspekten durch die Begegnung mit dem Anderen beschäftigt, wäre wohl nicht vollständig, wenn sie nicht einen gleichermaßen reflexiv-geleiteten Blick auf derlei Aspekte im eigenen Handeln richten würde. So stellt Kapitel 6 die Ergebnisse der Reflexion jener Bereiche und Momente vor, in denen die Begegnung mit dem Anderen mich selbst im Forschen hat innehalten lassen, um daraus schließlich neue Impulse im Prozess der Erkenntnisgewinnung $\mathrm{zu}$ ziehen. Insbesondere die interdisziplinäre Verortung und methodologische Vielfalt, die mein Handeln in den drei Teilprojekten dieser Dissertation geleitet haben, finden hier eine kritische Bertachtung. Auf diese Metaebene bezugnehmend bietet das letzte inhaltliche Kapitel den argumentativen Abschluss meiner Arbeit. Dieses 
Rahmenpapier ergänzend bietet Kapitel 9 Kurzvorstellungen der einzelnen Teilstudien an, bevor im Anhang die veröffentlichten Artikel nachlesbar sind.

Mit dem Aufbau und der Argumentationslinie dieses Rahmenpapiers bezwecke ich eine Zusammenführung der Einzelteile meiner Forschung als Ganzes. Verbindungslinien sollen verdeutlicht, dahinterliegende Verständnisse expliziert und die gewonnenen Erkenntnisse in ihrer Gesamtheit argumentiert werden. Im Zuge dieser Zusammenführung verzichte ich bewusst darauf, die einzelnen, in den Veröffentlichungen der Teilstudien gebotenen theoretischen Begründungen im Detail nachzuzeichnen oder dargelegte methodische Teilschritte zu wiederholen. Vielmehr skizziere ich die Summe der dahinterliegenden theoretischen und empirischen Erkenntnisse sowie den Rahmen ihrer interdisziplinären Verortung und ermögliche so, die Verwobenheit der Einzelforschungen meiner Arbeit bereits in Kapitel 2 nachzuvollziehen und ihre theoretische Anbindung auf einer höheren Komplexitätsstufe zu verorten.

Obgleich die vorliegende Arbeit durch vielfältigste interdisziplinäre Bezugspunkte geprägt wurde, möchte ich jene bildungswissenschaftlichen Werke hervorheben, die mich in meinem Bestreben, kulturwissenschaftliche und philosophische Identitätsverständnisse auf akademische Lernprozesse zu beziehen, inspiriert haben: In seiner Abhandlung zu Lernen in Beziehung umreißt Thomas Künkler (2011), welchen Wert ein relationales Lernverständnis haben kann und offeriert ein elaboriertes Geflecht kritischer Fragen zu vorhandenen Theorien und Konzepten des Lernens. Bereits zuvor hatte Käte Meyer-Drawe (2008) in ihren Diskursen des Lernens verdeutlicht, inwiefern ein breit angelegtes Ergründen menschlicher Lernprozesse und insbesondere ein Fokus auf Erfahrung als Kernelement derselben, als Weiterführung des pädagogischen Grundgedankens - Lernen als „Lernen von etwas durch jemand Bestimmten bzw. durch etwas Bestimmtes“ (ebd., S. 18) - genutzt werden kann. Schließlich waren es die Werke von Knud Illeris ${ }^{5}$ (2014) zum Konzept der Identität im Spiegel transformativen Lernens sowie von Mark Tennant (2012) zum lernenden Selbst, die mir die Erforschung des Anderen im Lernen unter Verwendung des Konzeptes der Alterität als logische Folge bestehender Erkenntnisse verdeutlichten.

Basierend auf Illeris‘ (2014, S. 1) Minimaldefinition von Identität als Konzept, über das wir uns selbst erfahren, uns zu Anderen und zur Welt in Relation setzen und aufgrund dieser Relationiertheit eine Identität ausbilden, ergibt sich die Nähe, gar Überschneidung zum Bildungsbegriff. Bildung, wenn sie mit Koller (2012, S. 9) als Prozess der Erfahrung verstanden wird, beschreibt jene Phänomene, in denen ein Mensch das eigene Wissen bearbeitet und schließlich mit einem erneuerten Verständnis von der Welt und sich selbst darin hervorgeht. ${ }^{6}$ In diesem Verständnis kann Bildung nicht nur als Erkenntnisprozess gefasst werden, sondern

\footnotetext{
${ }^{5}$ Die Arbeit von Illeris ist zum einen von Mezirows (2000) Fokus auf erfahrungsbasiertes Lernen geprägt und zum anderen (und maßgeblich) durch das Werk Peter Jarvis‘ (insb. 1992). Letzterer kombiniert soziologische, psychologische und philosophische Ansätze bei der Ausgestaltung einer holistischen Lerntheorie, die Lernen als Prozess begreift, aus dem eine Person nach Interaktion mit der Umwelt verändert hervorgeht.

${ }^{6}$ Fink (2013, S. 54) charakterisiert dieses Element menschlichen Lernens als reziprok und sagt „[...] when one learns about one's self, one almost inevitably learns about others, and vice versa."
} 
trägt in sich die Qualität eines „Denkwerkzeugs“ (Deimann, 2017), mithilfe dessen wir neu entdecktes Wissen ebenso wie inzwischen nicht mehr bewusste Zusammenhänge hinterfragen und (besser) verstehen können. Und genau hier findet sich ein Impuls wieder, der als weiteres Entstehungsmotiv meiner Forschung verstanden werden kann. Bei allem, was über Lern- und Bildungsprozesse in Bezug auf die Identität lernender Subjekte verhandelt wird, wird jenes Element vernachlässigt, das die Identität einer Person hervorzubringen vermag und - wie hier gezeigt wird - verwoben mit den jeweiligen Lernprozessen erfolgt: die Begegnung mit dem Anderen. Eine Lücke, die meine Arbeit schließt, indem sie das hier sinngebende Konzept der Alterität auf das Feld akademischer Lehr- und Lernprozesse anwendet. Das hierbei entstandene Modell zur Anwendung eines alteritätsbezogenen Lernverständnisses setzt gezielt dort an, wo der Mehrwert im Wahrnehmen unterschiedlicher Ebenen des Andersseins liegt. Es offeriert so ein bildungswissenschaftliches Instrument, das sowohl auf den Erwerb neuen Wissens ausgerichtet sein kann als auch darauf, beim Lernen und dem darin wahrgenommenen Anderssein etwas über sich selbst zu erfahren.

Schlussendlich ist es genau diese Begegnung mit dem Anderen, die den Mehrwert meiner Forschung in ihrer Gesamtheit möglich gemacht hat. Durch Rückbesinnung auf den Kern eines kulturwissenschaftlich-philosophischen Konzeptes wie dem der Alterität wurde hier bewusst dem disziplinär Anderen begegnet und sein Wert für das Verstehen akademischer Lehr- und Lernprozesse aus bildungswissenschaftlicher Perspektive erforscht. Das, was im eingangs angeführten Zitat Rortys bereits angedeutet wird, konnte so im Forschungsprozess nachempfunden und bestätigt werden: Der bildende Diskurs, der nichtnormal [sic!] ist, kann uns aus unserem alten, disziplinären Selbst herausführen. Für die vorliegende Arbeit hat dies ermöglicht, den bildungswissenschaftlichen Diskurs um neue Erkenntnisse zu bereichern.

\section{Theorie \& Erkenntnisinteresse - Wieso das wichtig ist}

Es sind fünf theoretische Grundlagen, die in diesem Forschungsprojekt verwendet und im Sinne des hier entstandenen alteritätsbezogenen Lernverständnisses weitergedacht wurden. Den Ausgangspunkt stellt - wie sollte es in einer Arbeit, die sich mit dem Lernen beschäftigt anders sein - das hier verwendete konzeptionelle Verständnis des Lernbegriffs dar. Begründet im Prozess des Lernens, dem die Begegnung mit etwas Neuem inhärent ist, wird im zweiten Teil dieses Kapitels skizziert, auf welchen Ebenen auch weitere Begegnungen im Lernen möglich sind. Da sie alle mit der Wirkung und Entstehung von Reflexionsimpulsen verwoben sind, wird der Bedeutung der Reflexion in meiner Arbeit besondere Aufmerksamkeit geschenkt. Im zugehörigen Kapitel 2.3 stelle ich zudem eine Erweiterung bestehender Reflexionsverständnisse vor, um die in ihnen vorhandenen Qualitäten für das Lernen adäquater zu fassen. Nicht nur für das Lernen, auch für die Konstruktion von Identitäten bedarf es den Prozess der Reflexion. Sie ist es, durch die ein Individuum den Impuls erhält, sich (er)neu(t) zu verorten. Inwiefern die Identität einer Person sowie ihre Sozialisation in einen Kontext - wie dem der Wissenschaft - untrennbar mit Prozessen des Lernens 
verbunden sind, wird in einem weiteren Schritt argumentiert und so der letzte relevante Bereich zur Einführung des Konzeptes der Alterität in den bildungswissenschaftlichen Diskurs ermöglicht. Gemeinsam untermauern diese theoretischen Zugänge, weshalb eine tiefgehend-empirische Auseinandersetzung mit dem Konzept der Alterität für das Lernen bislang eine Forschungslücke dargestellt hat.

\subsection{Konzept(e) des Lernens}

Wenn Menschen lernen, dann geschieht dies durch ein Zusammenspiel verschiedenster kognitiver, affektiver und meta-kognitiver Prozesse, die in Verbindung zu vorhandenen Wissenskonzepten, Einstellungen und Dispositionen wahrgenommene Informationen verarbeiten und aus ihnen neue, für das lernende Subjekt relevante Erkenntnisse ziehen. ${ }^{7}$ Das Was und Wie des Lernens sind hierbei geprägt durch ihre Situiertheit innerhalb eines bestimmten Kontextes. Dieser beeinflusst durch seinen sozialen Charakter nicht nur das Setting, sondern auch den Inhalt des Lernens. Gleichsam adressiert er die soziale Rolle ${ }^{8}$, die das Individuum beim Lernen einnimmt, im akademischen Kontext beispielsweise als Hochschullehrende*r, Student*in oder Forscher*in. So kann Lernen verstanden werden als Prozess der subjektiven Informationsverarbeitung ${ }^{9}$ und Wissensaneignung innerhalb eines durch soziale, mitunter institutionelle Aspekte beeinflussten Kontextes. ${ }^{10}$

Lernen erfolgt weiter stets in Bezug auf Etwas, seien es bereits erworbene Verhaltensmuster oder begründete Wissenskonstrukte, gemachte Sinneserfahrungen oder durchlebte Situationen. ${ }^{11}$ Komprimiert auf drei Prozesselemente kann Lernen, das als bedeutungsvoll ${ }^{12}$ gilt, gefasst werden als die Zusammenführung abstrakter Informationen mit konkret erfahrenen Situationen durch den Prozess der Reflexion. Auf dieser Abstraktionsebene überschneidet sich das hier verwendete Konzept des Lernens mit denen zur Kompetenzentwicklung. ${ }^{13}$ Auch in diesen ist es die abstrakte Wissensbasis individueller Fähigkeiten und Fertigkeiten, die sich im Handeln zeigt und so Situationen entstehen können, in denen eine Person sich durch Reflexion als kompetent wahrnimmt. Während das

\footnotetext{
${ }^{7}$ Vgl. grundlegend Entwistle (2009), Göhlich \& Zirfas (2007) sowie Weimer (2002).

${ }^{8}$ Ergänzend zur sozialen Rolle, die eine Person in einer bestimmten Situation einnimmt, können sozial konstruierte, (selbst)zugeschriebene Kategorien wie beispielsweise Alter oder Geschlecht für das Lernen in einer bestimmten Rolle relevant sein. Ein Fokus, der in meiner Argumentation bewusst ausgespart wird.

${ }_{9}^{9}$ Die hier formulierten Grundannahmen stehen in bewusster Relation zu den formalen Aspekten der

Kommunikation, wie sie bereits 1967 von Watzlawick \& Beavin hervorgehoben wurden: Dass in kommunikativen

Prozessen neue Informationen mit vorhandenem Wissen interagieren und im Zuge ihrer Begegnung ein

Aushandlungsprozess stattfindet (ebd., S. 4).

10 Aus lernpsychologischer Sicht fokussiert meine Arbeit jene Seite des Lernens, die sich mit Wissenserwerb befasst. Lernen in der Qualität als Prozess der Verhaltensänderung wird in meinem Zugang zwar nicht als Ausgangspunkt genommen, spiegelt sich in der Analyse in Kapitel 4 jedoch als relevant wieder.

11 In meiner Arbeit wird die Relevanz affektiver Aspekte im Lernen nicht expliziert. Eine Anwendung des hier entwickelten alteritätsbezogenen Lernverständnisses auf emotionale Bereiche des Lernens stellt für mich vielmehr einen nächsten Schritt dar (vgl. Kapitel 5).

${ }^{12}$ Lernen, das bedeutungsvoll ist (significant learning), grenze ich in Anlehnung an Entwistle (2009) und Fink (2013) von jenem Lernen ab, das sich durch reines Memorieren kennzeichnet (passive learning).

13 Vgl. hierzu die theoretischen Erläuterungen in Teilstudie C (Thielsch, 2019b) sowie weiterführend Klinger et al. (2013), Olsson et al. (2012) sowie Trautwein (2013).
} 
empfundene Kompetent-Sein ${ }^{14}$ Ergebnis dieses Zusammenspiels ist, fokussiert die Kompetenzentwicklung zunächst das Potential, das es individuell zu erkennen und auszubilden gilt. $^{15}$ Ein solcher Prozess der Kompetenzentwicklung beruht auf einer Vielfalt von Lernprozessen, die ihrerseits erfolgen können, ohne zwangsläufig in Kompetenzentwicklung zu münden. Für die hier geführte Argumentation verstehe ich Lernen und Kompetenzentwicklung als zwei im Kern vergleichbare Konzepte, die - trotz ihrer unterschiedlichen Grade der gegenseitigen Abhängigkeit - theoretisch in einem semantischen Feld zu verorten sind. In ihrem gemeinsamen Fokus auf die Prozesshaftigkeit und insbesondere die Bedeutung der Erfahrung im Lernen stellen sie die konzeptuelle Grundlage der weiteren Überlegungen dar.

Der hier verwendete Begriff der Erfahrung ${ }^{16}$ impliziert ein Erleben, das das eigene Weltverständnis zu verändern vermag ${ }^{17}$ und so Teil subjektiven Denkens wird. Gleichsam entstehen Erfahrungen in Relation zu vorhandenen Wissenskonstrukten einer Person und finden somit (wie alles Lernen) eingebettet in soziale Diskurse statt. Der soziale Charakter menschlichen Lernens und die Individualität dieser Prozesse beschreiben so das Fundament meiner Forschung, in der das lernende Subjekt ins Zentrum der Betrachtung tritt und aus erlebten Situationen Wissen-konstruierende Erfahrungen erzeugt.

Das für diese Arbeit herangezogene konzeptionelle Verständnis von Lernen möchte ich in Abbildung 1 zusammenfassen und veranschaulichen. Als Erweiterung eines von Illeris ${ }^{18}$ (2014, S. 34f.) skizzierten Prozessmodells des Lernens im Sinne des Wissenserwerbs ${ }^{19}$ und ergänzt um die Vielschichtigkeit subjektiver Identitäten finden sich in dieser Abbildung alle für den weiteren Verlauf der Argumentation relevanten Ebenen des Lernens wieder.

Eingebettet in einen spezifischen sozialen Kontext wird hier Lernen als individuelles Phänomen verortet, das durch eine Interaktion und der daraus erfolgten Erfahrung mit der Umwelt initiiert wird und - wie im Modell dargestellt - auf zwei parallelen Ebenen stattfindet. In Kombination ergeben sich aus diesen Grundparametern jene Faktoren, die individuelles Lernen beeinflussen.

14 Vgl. zum Begriff der Kompetenz u.a. Rychen \& Salganik (2001), Tremp (2012) und Weinert (2001).

15 Illeris (2014, S. 115ff.) verdeutlicht die Relevanz dieses Unterschieds mit Blick auf Prozesse der Kompetenzentwicklung und -Kompetenzmessung. Der Fokus erfolgreicher Kompetenzentwicklung solle nicht darauf liegen, dass eine Person bereits gezeigt hat, was sie kann, sondern darauf, was sie in einer unbekannten Situation zu tun in der Lage wäre.

${ }^{16}$ In Teilstudie C habe ich in Anlehnung an Echterhoff (2013), Gruber \& Rehrl (2005) und Meyer-Drawe (2003) den Begriff der Erfahrung ,als eine auf Handlung basierende Information [gefasst], die durch subjektive Wahrnehmung erzeugt wird und als episodische Kenntnis über die Qualität und Verortung bestehenden Wissens innerhalb eines spezifischen Kontextes Auskunft gibt“ (Thielsch, 2019b, S. 163).

${ }_{17}$ Vgl. hierzu Bredella (2010, S. 8), der hervorhebt: „Wer eine Erfahrung macht, tritt aus dem Fluss der Erfahrungen heraus, sodass die Erfahrung seine Weltsicht verändern kann.“

18 Bereits in 2007 hatte Illeris in seinem Werk „How we learn“ als drei Grunddimensionen des Lernens die Situiertheit, den Inhalt sowie den Modus des Lernens benannt.

${ }^{19}$ Da meine Arbeit auf Lernen als Wissenserwerb fokussiert und nicht auf Lernen als Verhaltensänderung, ist letztere im Modell nicht explizit erfasst. 


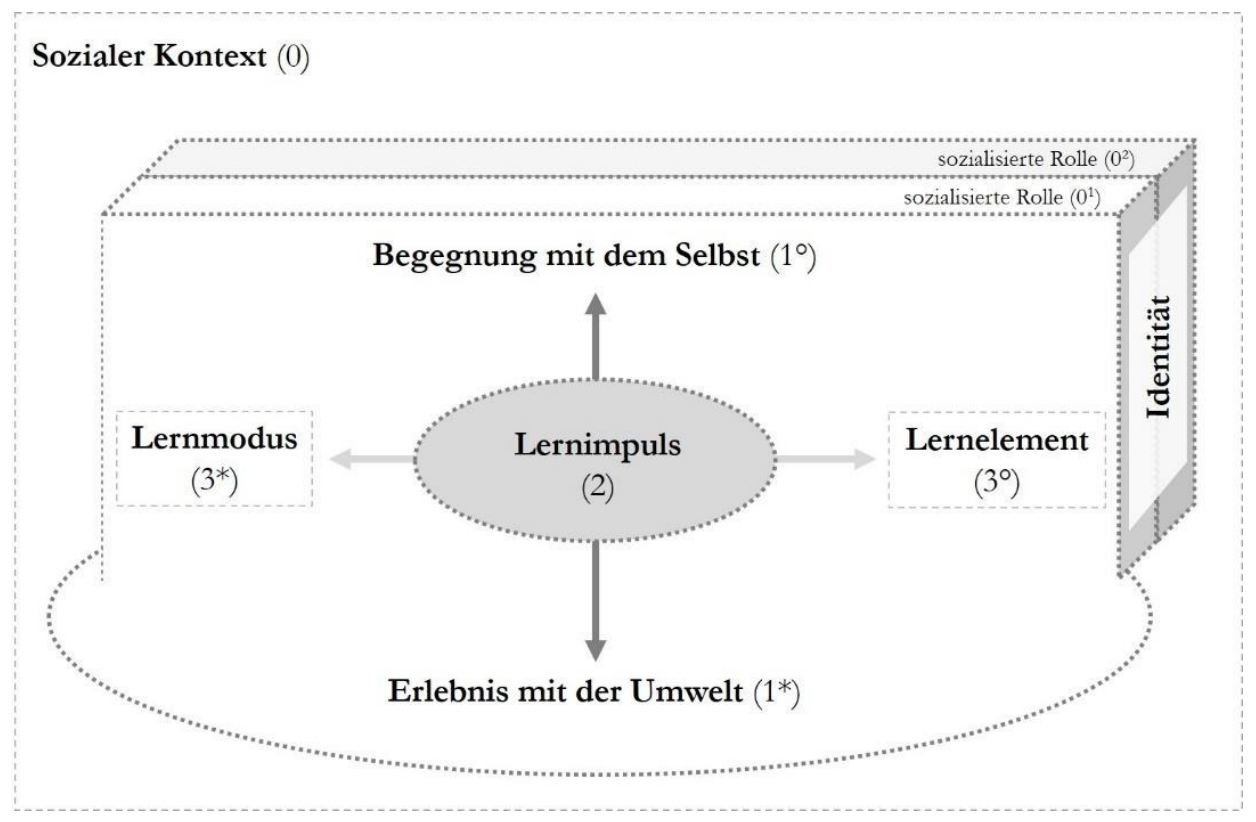

Abb. 1: Prozessmodell des Lernens

Die Begegnung des Selbst $\left(1^{\circ}\right)$ mit der belebten und unbelebten Umwelt $\left(1^{*}\right)$ erzeugt einen Lernimpuls (2), der Zweierlei auslöst: Zum einen, dass sich der Modus des Lernens ( $\left.3^{*}\right)$ ergibt, somit das Wie, das Wozu und das Wobin, zum anderen die bewusste Auseinandersetzung mit der wahrgenommenen Information sowie ihre Verarbeitung $\left(3^{\circ}\right)$, folglich das $W$ as bzw. das Lernelement. Dieses $W$ as kann hierbei gleichsam ein fehlendes Abstraktes oder Konkretes sein oder auch der Impuls zur Reflexion, die beides verbindet. Als Besonderheit des Modells ist hervorzuheben, dass es eine mehrschichtige Verortung des Lernens ermöglicht. Gerahmt in einem spezifischen sozialen Kontext (0) erfolgt Lernen stets in Relation zu einer bestimmten, in der gegebenen Situation gelebten sozialen Rolle $\left(0^{1}, 0^{2}, 0^{3}\right.$ etc.) einer Person. Die Rollen, in die man hinein sozialisiert ist, nehmen so Einfluss auf den Lernprozess als Ganzes.

Lernen verstehe ich als einen relationalen Prozess, der weder isoliert von individuellem Vorwissen noch unabhängig sozialer Kontexte erfolgt und innerhalb dessen ein Individuum durch das Ausüben einer sozialen Rolle eine wahrgenommene Information verarbeitet, die zuvor durch einen externen Impuls qualitativen Mehrwert erhalten hat.

Die Stärke dieses vielschichtigen Modells ergibt sich hinsichtlich des Themas der vorliegenden Arbeit insbesondere durch drei Aspekte. Für das Feld des menschlichen Lernens unterstreicht es dreierlei: die Individualität im Lernprozess durch Beachtung des Konzeptes der Identität, die Relevanz des sozialen Kontextes durch Beachtung des Konzeptes der Sozialisation sowie die Notwendigkeit des Lernimpulses durch Beachtung des Konzeptes der Reflexion. Jedem der hier angesprochenen Konzepte soll in den nächsten Abschnitten dieses Rahmenpapiers Aufmerksamkeit gewidmet werden, um ihre Bedeutung für das avisierte 
alteritätsbezogene Lernverständnis herzuleiten und um ihr Ineinandergreifen fortan mitdenken zu können. Auch das Konzept der Alterität findet sich in diesem Modell bereits implizit wieder. Sind es doch Begegnungen mit Etwas, die den Impuls zum Lernen erzeugen und mit denen ein Subjekt interagiert. Ein Abgleich, der sich - wie ich in den nächsten Abschnitten erläutern werden - in seiner Wirkungskraft in Relation zum Konzept der Alterität begreifen und analysieren lässt.

\subsection{Begegnungsprozesse im Lernen}

Begegnungsprozesse sind Bestandteil des Lernens und dies auf kognitiver wie auch affektiver Ebene. Im Lernen begegnen wir dem, was wir bereits über ein Thema oder einen Kontext wissen. Wir begegnen weiter eigenen und anderen Erwartungen an eine Lernsituation oder ihren Inhalt. Im Lernen sind wir geleitet durch lernbiographische Erfahrungen ${ }^{20}$ sowie durch die Strukturen institutioneller Kontexte, in denen wir uns in einen Lernprozess begeben. Dass Situationen des Lernens von einer Vielzahl von Begegnungen durchzogen und geprägt sind, stellt für die Einordnung meiner Arbeit einen Grundstein dar. Ich werde daher kurz umreißen, welche Erkenntnisse über Begegnungen in Lernprozessen und in Relation zur Anwendung des Konzeptes der Alterität für die hier präsentierte Forschung relevant sind.

In Anlehnung an Schloos (2000, S. 8f.) lassen sich Begegnungen im Lernen in drei Dimensionen einordnen: Zunächst ergibt sich im Lernen die Begegnung mit einem (neuen) Inhalt. Eine Person entdeckt Informationen, die bislang unbekannt oder anders verstanden wurden und erweitert in Abgleich dazu das eigene Wissen. In formalen Lernkontexten erfolgt dies oft in Verbindung mit der zweiten Dimension, der Begegnung mit anderen Personen. Sowohl Lehrende als auch andere Lernende sind es, deren Perspektiven und Einstellungen mitprägen, was eine Person im Lernen erfährt. Die Anderen werden Teil der Lernerfahrung. Schließlich - und hier wird die Verbindung zum Konzeptpaar Identität und Alterität deutlich - begegnen wir uns im Lernen selbst. Diese dritte Dimension nach Schloos beschreibt, dass im Zuge der internen Aushandlung darüber, was, wann, wie und auch mit wem wir lernen, auch ein Lernen über uns selbst erfolgen kann.

Die hier vorgeschlagenen Begegnungsdimensionen wurden kürzlich und in Verbindung mit diesem Forschungsprojekt auf den Hochschulkontext appliziert und mögliche sowie vorhandene Begegnungen in verschiedenen formalen Lehrsettings der Universität analysiert (vgl. Brandt et al, 2019 i. E.). ${ }^{21}$ Die hier argumentierte Erkenntnis, dass den in der universitären Lehre inhärenten Begegnungsprozessen ein lernförderliches Potential zugesprochen werden kann, zielt in erster Linie darauf ab, Lehrende für dieses Potential zu sensibilisieren und es didaktisch mitzudenken. Weiter wird durch diesen Übertrag (ebd.) die Verbindung von

${ }^{20}$ Vgl. Arbeit zur Relevanz der Lernbiographie, u.a. Alheit \& Dausien (2009) und Kiehne (2015).

${ }^{21}$ Beitrag mit dem Titel „Begegnungen im Kontext Hochschullehre - Entdecken, reflektieren, nutzen“", der im Rahmen des Tagungsbandes zur Jahrestagung der Deutschen Gesellschaft für Hochschuldidaktik (dghd) 2017 in Köln im Jahr 2019 erscheinen wird. 
Reflexionsprozessen und der Begegnung mit dem Anderen in der universitären Lehre unterstrichen.

Im Lernen können wir stets auch dem begegnen, das uns herausfordert, uns innehalten lässt, unsere Erwartungen widerlegt, unsere Sinnkonstrukte hinterfragt und so das Lernen (neu) initiiert. Ein Umstand, der - wie dieses Rahmenpapiers nachzeichnet - durch das Konzept der Alterität erklärbar ist und dafür auf die Wirkungsmacht der Reflexion zurückgreift.

\subsection{Die Bedeutung der Reflexion}

Lernen braucht Reflexion. Durch Reflexion wird Wahrgenommenes ergründet und erlangt Bedeutung, wird abstrakte Information in Relation zu konkret erlebten Situationen gesetzt und werden vorhandene Wissenskonzepte hinterfragt und bearbeitet. Durch Reflexion kann ein Lernimpuls die Qualität erhalten, um auf bestehende Sinnkonstrukte einzuwirken.

Reflexion verstehe ich als subjektive, zeitlich begrenzte Aktivität des Hinterfragens von Informationen, die für das reflektierende Individuum auf verschiedenen Bedeutungsebenen relevant sein können: hinsichtlich bestehender Wissenskonstrukte über die Welt oder individuelle Selbstverständnisse (eines gelebten Rollenbildes) sowie hinsichtlich avisierter oder bereits getätigter Handlungen in Verbindung zur Beschaffenheit des jeweiligen Settings. Durch Reflexion werden diese Informationen im Zuge eines mehrstufigen (Denk- und Interpretations-) Prozesses systematisch und in Abgleich mit den genannten Bedeutungsebenen hinterfragt und neu gedeutet. Reflexion ist somit zielgerichtet-bewusstes Ergründen, das danach strebt, eine für das Individuum noch verborgene Deutung vorhandener Information(en) zu ermitteln. ${ }^{22}$

Als einem der oben angeführten Prozesselemente des Lernens kommt der Reflexion die Aufgabe zu, das Abstrakte einer Information mit der Erfahrung aus einer konkreten Situation in Verbindung zu setzen und beidem in Ergänzung zueinander einen neuen Wert für das Wissenskonstrukt einer Person zu geben. ${ }^{23}$ Weiter kann sie antizipierend ausgerichtet sein und basierend auf vorhandenem Wissen über vergleichbare Situationen und Kontexte im Vorfeld einer konkreten Erfahrung und diese vorbereitend erfolgen. Innerhalb eines Lernprozesses kann Reflexion so verschiedene Positionen einnehmen, wie Abbildung 2 zusammenfasst. ${ }^{24}$ Schematisch wird hier dargestellt, in welcher Abfolge die drei Prozesselemente des Lernens -

22 Im bildungswissenschaftlichen Diskurs ist die Relevanz der Reflexion oder des reflective thought verwoben mit der Arbeit Deweys, insbesondere seiner Abhandlung „How we think“ (1910). Hier bereits hebt er hervor, dass Reflexion nicht ungeordnet, sondern bewusst und schrittweise erfolgt (ebd., S. 2) und im Individuum durch ,a state of perplexity, hesitation, doubt“" (ebd., S. 9) ausgelöst wird. Ashwin et al. (2015, S. 43ff.) weisen zudem darauf hin, dass Dewey durch seine Unterscheidung zwischen routine action und reflective action die Grundlage legte, auf der ein reflexiver Lehransatz begründet werden konnte. Vgl. zur Bedeutung der Reflexion weiter Boud \& Walker (1990 und 1998), Fink (2013) sowie Jenert (2008).

${ }^{23}$ Ein Phänomen, das Argyris \& Schön (1974) als double loop learning bezeichnen. Im Gegensatz zum single loop learning, in dem es um Aneignung neuen Wissens geht, wird hier avisiert, bestehendes Wissen oder den Prozess seines Erwerbs zu hinterfragen (vgl. Hilzensauer, 2008, S. 4f.).

${ }^{24}$ Mezirow (1991) unterscheidet drei Typen der Reflexion: Bezogen auf den Inhalt (content reflection), den Prozess (process reflection) sowie die dahinterliegenden Bedeutungen (premise reflection) (vgl. Cranton, 1996, S. 81f.). 
eine abstrakte Information (A), eine konkret erfahrene Situation (K) sowie die Reflexion (R) innerhalb eines Lernprozesses verbunden werden können.

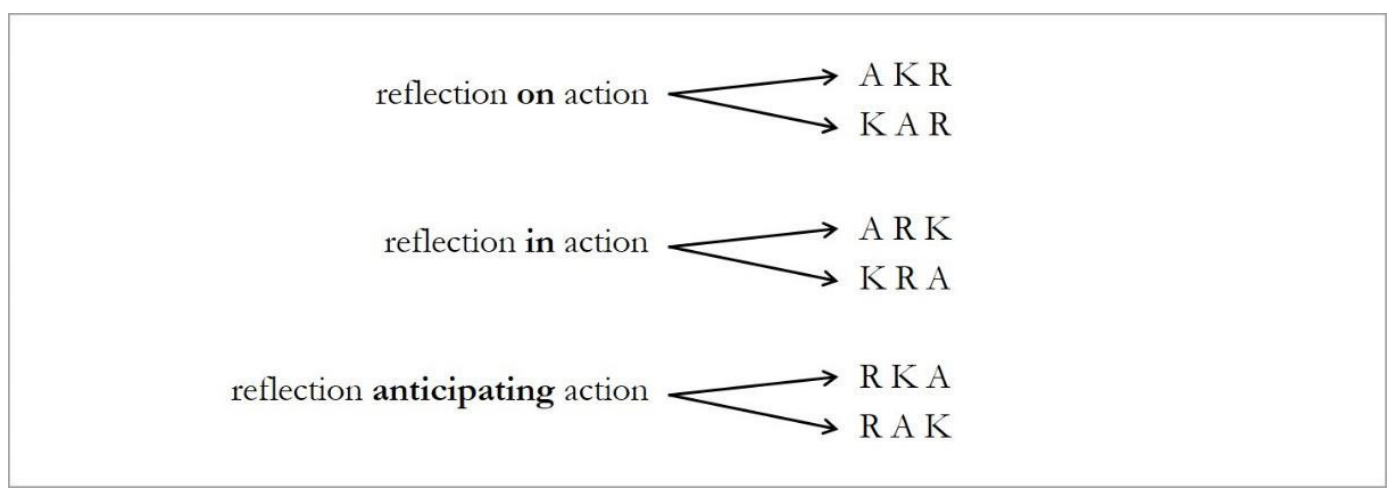

Abb. 2: Reflexion im Lernprozess

Während die Argumentation der ersten beiden hier skizzierten Bereiche - reflection on action und reflection in action - insbesondere auf die Arbeiten Donald Schöns ${ }^{25}$ und seine Überlegungen des professionellen Handelns zurück gehen, findet sich letztere - reflection anticipating action erst in späteren Abhandlungen ${ }^{26}$ expliziert. Zusammen verdeutlichen diese drei die vielfältigen Wirkungsrichtungen der Reflexion und unterstreichen ihre Relevanz; sei es aus Perspektive des Lernens, des Lehrens oder des Lehren Lernens.

Reflexion kann als Kernelement dessen verhandelt werden, was Lernen initiiert. Denn trotz aller Erkenntnisse darüber, wodurch sich erfolgtes Lernen erfassen lässt, ist sein Ursprung nach wie vor aufgrund seiner Kontextabhängigkeit und Individualität wenig greifbar. Lernen, so Meyer-Drawe (2003, S. 509), sei vergleichbar mit dem Aufwachen. „Man ist dabei, aber nicht als konstituierendes Subjekt“ (ebd.). Durch den gezielten Einsatz der Reflexion, insbesondere der antizipierenden, kann ein Individuum dieses Aufwachen rekonstruieren oder ihm vorgreifen, seine qualitativen Parameter ergründen und sie für zukünftige Lernprozesse bewusst einsetzen. Sie hilft zu benennen, was genau gekonnt und verstanden wurde, was dafür notwendig war und welche Bedeutung beides für die individuelle Lernbiographie haben kann. In ihrer lernstrategischen Funktion hat Reflexion somit die Aufgabe, routiniertes Handeln zu explizieren und implizit Bewusstes zu begründen. Zudem wird in Erweiterung hierzu dann von kritischer Reflexion ${ }^{27}$ gesprochen, wenn dieses Ergründen und Interpretieren nicht alleine auf $V$ erstehen, sondern auf Verändern abzielt. Der Blick richtet sich hier auf zukünftiges Handeln und den Wunsch, das eigene Verhalten in einer speziellen Situation sowie das hierfür relevante Wissen zu erweitern.

25 Seine zwei zentralen Werke sind "The Reflective Practitioner. How rofessionals think in action" (1983) sowie "Educating the Reflective Practitioner. Toward a New Design for Teaching and Learning” (1987).

26 Vgl. van Manen (1991) und Wiemer (2012).

${ }^{27}$ Futterer (2012, S. 172) unterstreicht, dass in der Regel (aber nicht ausschließlich) dann von kritischer Reflexion gesprochen wird, wenn es der Problemlösung dient. Hier sei die Intention, bislang eingenommene Perspektiven sowie die Parameter eigener Wissenskonstrukte und Einstellungen sinnvoll zu erweitern. Sinnvoll in dem Sinne, als dass sie die Gelegenheit wirkungsvoll(er)en Handelns in einem bestimmten Kontext ermöglichen. 
All dies greift für ein Verständnis des Konzeptes der Reflexion meines Erachtens nach jedoch zu kurz. ${ }^{28}$ Denn neben der hier skizzierten Qualität als zielgerichtete Handlung im Lernprozess ist der Reflexion im menschlichen Lernen noch eine weitere Eigenschaft inhärent. Wir wissen, dass sie durch einen nicht bewusst intendierten Impuls ausgelöst werden kann, der sich durch die in Kapitel 2.1 elaborierte und in Abbildung 1 skizzierte Interaktion mit der Umwelt ergibt. Kommt eine Routine ins Stocken, wird eine Einstellung herausgefordert oder ein bestehendes Wissenskonstrukt angezweifelt, kann im individuellen Lernen der Prozess der Reflexion initiiert werden. Es entsteht eine Unsicherheit, die sich in Relation zum lernenden Subjekt sowie dem jeweiligen sozialen Kontext ergibt.

Somit kann und sollte bei der Erforschung von Reflexion Zweierlei unterschieden werden: Reflexion aufgrund eines nicht-intendierten Lern-Impulses $\left(\mathrm{R}^{\mathrm{LI}}\right)$ einerseits und Reflexion als bewusst-lernstrategische Handlung $\left(\mathrm{R}^{\mathrm{LS}}\right)$ andererseits. Mit Blick auf diese zwei initialen Reflexionsqualitäten wäre das oben vorgestellte Modell zur Positionierung der Reflexion im Lernen zu erweitern. Der Grundsatz, dass es ein Zusammenspiel von abstrakt-theoretischer Information, konkret-erfahrener Situation und Reflexion zum Durchlaufen eines Lernprozesses braucht, bleibt von dieser Ergänzung einer weiteren Reflexionsqualität unberührt.

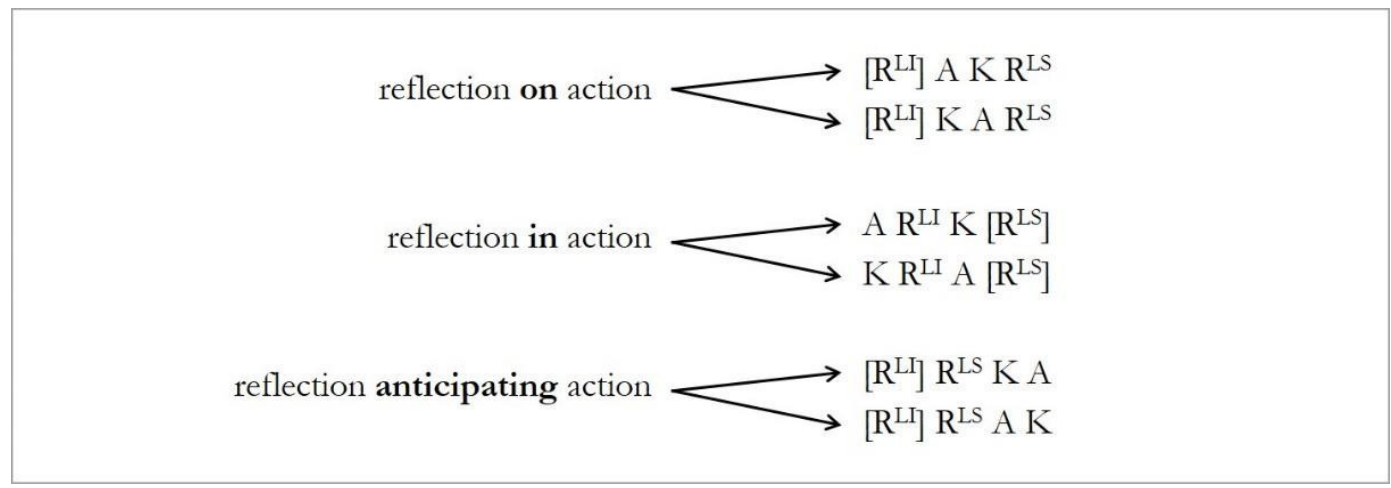

Abb. 3: Zweierlei Qualitäten von Reflexion im Lernprozess

Wie in Abbildung 3 verdeutlicht, ist lediglich je ein weiteres Element als Möglichkeit in die bestehenden Abfolgen eingeflossen: das Element des Wodurch, des Impulses. Eine Relevanz kann dies für alle dargestellten Abfolgen im Lernprozess haben. Am bedeutsamsten erscheint die Unterteilung jedoch im Bereich der reflection in action. Was genau ist es, dass eine Person in einer Handlung innehalten und das eigene Tun überdenken lässt? Ein Reflexionsimpuls, der nicht antizipiert wurde, sondern dem man unerwartet begegnete. Ein solcher Impuls kann auch der Auslöser sein, um sich überhaupt erst in einen Prozess der reflection anticipating action hinein zu begeben oder, nach Beenden einer Situation, diese erneut zu überdenken und dann daraus Schlüsse, weiterführende Interpretationen zu generieren. Im Unterschied zur Reflexion

${ }^{28}$ Hier gehe ich einher mit der Annahme von Light et al. (2009, S. 59), die die Beziehung zwischen einer Erfahrung und der Reflexion derselben als „,komplexer“ erachten, als „oftmals gedacht wird“. 
als lernstrategisches Element kann die Reflexion, die durch einen nicht-intendierten Lernimpuls initiiert wird, Lernprozesse befördern, die ansonsten möglicherweise nicht in Gänze durchlaufen worden wären.

Beide Qualitäten der Reflexion sind gleichermaßen relevant im Verlauf eines Lernprozesses, beide sind eng verwoben mit der lernenden Person. Der Unterschied beider liegt somit nicht in ihrer Verlaufsstruktur, sondern in ihrem Ursprung und dessen Bezugsgrößen. Während Reflexion als nicht-intendierter Impuls den sich stetig weiterentwickelnden Bereich individueller Lernbiographien und (Vor)Wissenskonstrukte prägt, ist sie als Lernstrategie maßgeblich damit verbunden, wer genau es ist, der lernt und reflektiert, welche sozialen Strukturen das Sein dieses Individuums beeinflussen. Hier zu unterscheiden ist meines Erachtens und insbesondere für die vorliegende Arbeit hoch relevant.

Das Konzept der Reflexion ist in beiden Qualitäten für das Begreifen menschlichen Lernens von Bedeutung. Das Mehr der hier präsentierten Forschung ergibt sich jedoch vordergründig durch Reflexion in ihrer Qualität als nicht-intendierter, bildungsrelevanter Impuls, der bestehende Weltverständnisse hinterfragt und Subjekte veranlasst, sich in ihren Sinnkonstruktionen (kognitiv oder affektiv) neu zu verorten. Ein solcher Reflexionsimpuls ist es, der in der Begegnung mit dem Anderen ausgelöst wird.

Um diese Annahme theoretisch zu konkretisieren, bedarf es zunächst der Klärung weiterer zentraler Begriffe: der akademischen Sozialisation sowie der Identität. „Who we are, emotionally and intellectually, is key to our reflective [...] practices" formulieren Ashwin et al. (2015, S. 45) prägnant. Somit ist die Auseinandersetzung mit dem, was die Identität einer lernenden Person ausmacht, nicht nur Schlüssel zum Verstehen ihrer reflexiven Handlungen, sondern auch für den hier vorgestellten Forschungskontext als Ganzes.

\subsection{Das Ich im (akademischen) Lernen}

Die Identität eines Individuums befindet sich in einem kontinuierlichen Prozess der Konstruktion, Dekonstruktion und Rekonstruktion. ${ }^{29}$ Verstanden als (psycho)soziales Konzept fällt der Identität eines Menschen die Aufgabe zu, dem Selbst ${ }^{30}$ einen Sinn im jeweiligen Kontext zu geben. ${ }^{31}$ Diesem Grundverständnis innewohnend ist die Überzeugung, dass jede menschliche Identität eine Vielzahl von Rollen und Selbstverständnissen in sich

\footnotetext{
${ }^{29}$ Barnett \& Di Napoli (2008, S. 6) formulieren es wie folgt „,We see identities [...] as a historical process of construction, deconstruction and reconstruction, three movements that we see at the heart of any identity dynamics." Vgl. zudem Illeris (2014), Taylor (2008) und Tennant (2012).

${ }_{30}$ Mit dem Begriff des Selbst wird ein In-Beziehung-Treten mit sich selbst bezeichnet. Die zentrale Abgrenzung zum Begriff der Identität besteht darin, dass diese sich in der Beziehung zu (dem) Anderen begründet. Beide sind durch ihre subjektive und soziale Einbettung miteinander verbunden (vgl. Illeris, 2014; Tennant, 2012).

31 Taylor (2008, S. 28) unterstreicht gleichsam die Relevanz, die das Einnehmen einer bestimmten Rolle für den Kern individueller Identität haben kann: „For individuals, roles give rise to context-specific opportunities to express, and even develop, personal identity."
} 
vereinigt. Im Sein offeriert sie dem Individuum einen verbindenden und bedeutungsgebenden Kern, der das Wechseln zwischen den verschiedenen Rollen leitet. Identität ist somit kein stabiles, auf Selbstdefinition beruhendes Ganzes, sondern vielmehr eine immerwährende, historisch-kontextuelle Positionierung. Der jeweilige soziale Kontext offeriert hierbei den Impuls, um eine bestimmte Rolle einzunehmen und gemäß der Parameter ihres jeweiligen Bezugssystems zu handeln. So interagiere ich in meiner Rolle als Akademikerin auf andere Art und Weise mit der Umwelt, als ich es in meiner Rolle als Freundin oder Tochter täte. Gleichsam nehme ich je nach Rolle Lernimpulse anders wahr und verarbeite diese in bestimmten Modi. Die Grundannahme, dass Lernen sozial eingebettet erfolgt, kann vor dem Hintergrund des Identitätsdiskurses eine weitere Deutungsebene erhalten.

Der hier verwendete postmoderne Identitätsbegriff, der in seiner Grundannahme die Pluralität von Selbstverständnissen als Teil eines sich immer neu entwerfenden Identitätsbildes veranschlagt, misst dem Prozess der (Selbst)Reflexion große Bedeutung zu und setzt sie in das Zentrum theoretischer Auseinandersetzungen zu dieser Thematik. ${ }^{32} \mathrm{Um}$ in einem Kontext wirkungsmächtig handeln zu können, braucht das Individuum einen kontinuierlichen Abgleich mit (sowie Vergewisserung) der eigenen Identität und der darin zusammenwirkenden Rollen. Es ist dieses fortwährende (Neu)Entdecken individueller Sinnhaftigkeit, in der die Parallele zwischen dem Konzept der Identität und dem des Lernens deutlich wird. Beide sind in ihrem Wirken relational, beide konstituieren und verändern einander fortwährend. Illeris (2014, S. 64) beschreibt diese Verbindung wie folgt: „[] dentity is created, developed and changed through learning - which all takes place in an interplay with the innate dispositions that are integrated in the learning process and thereby can influence the learning as well as the identity".

Jede Erfahrung offeriert Lernimpulse und so Erkenntnisse, die bestätigend oder hinterfragend auf die eigene Identität wirken können. ${ }^{33}$ Gleichsam ist es die Identität, die beeinflusst, welches Lernverhalten in einer Situation gewählt, auf welches Wissenskonstrukt zugegriffen wird und welche Einstellungen das Individuum dabei leiten. Es sind folglich die individuellen Wissenskonstrukte und Überzeugungen eines Individuums in einer bestimmten, situativ eingenommenen Rolle, die beeinflussen, ob und wie intensiv ein Lernimpuls wahrgenommen wird. Lernen und Identität teilen sich denselben Kern; ein Phänomen, das das eine verändert, wirkt auch auf das andere.

Die Aufgabe der Identität ist es nun, als Quelle der Orientierung im Wechsel zwischen den verschiedenen sozialen Rollen eines (lernenden) Subjektes zu dienen. Sie organisiert die Pluralität an Rollen, die ein Subjekt aufgrund seiner sozialisierten Zugehörigkeiten zu einer oder auch mehreren Gruppen innehat. Die Stärke individueller Identität kann sich folglich in ihrem Können offenbaren, eine bestimmte Rolle passend zum jeweiligen Kontext auszuleben, ohne damit in Widerspruch zu ihrem eigenen (Selbst)Konzept zu treten, sondern vielmehr als Ausdruck desselben zu wirken.

32 Vgl. Barnett \& Di Napoli (2008) sowie Eickelpasch \& Rademacher (2004).

${ }_{33}$ Diese Annahme wurde jüngst erneut durch eine qualitative Langzeitstudie im Bereich des Sprachenlernens bestätigt (vgl. Takkac Tulgar, 2019). 
Der Ursprung jeder dieser Rollen, die ein Mensch als die eigenen auslebt, liegt im Prozess der Sozialisation. Wenn ich in dieser Arbeit von Sozialisation spreche, dann beziehe ich mich auf sozial- und kulturwissenschaftliche Diskurse, die damit auf einen Prozess verweisen, im Zuge dessen ein Individuum die Werte, Regeln und Sinnstrukturen einer sozialen Gemeinschaft bzw. eines sozialen Kontextes erfährt, in das eigene Wissenssystem übernimmt und als (zunehmend unhinterfragtes) Leitmotiv der eigenen Handlungen anwendet. ${ }^{34}$ Der Wunsch, innerhalb eines Kontextes wirkungsmächtig handeln zu können, kann als sozialisiertes Mitglied der jeweiligen Gruppe erfüllt werden. Der Zugang zu dieser Wirkungsmacht erschließt sich dem Individuum im Zuge der Sozialisation jedoch nicht durch passives Nachvollziehen, sondern durch aktive Teilhabe, durch die Praxis des sich Einfindens. ${ }^{35}$ Für das System Wissenschaft ist dies, so Rhein (2015), mit dem Modus des Studierens verzahnt. Dieser bedeutet nicht nur, Wissenschaft als abstraktes System von Aussagen zu erlernen, sondern gleichsam, sich Wissenschaft als Praxis des „Erklärens und Verstehens“ (ebd., S. 354) zu erschließen. Das hier implizierte Einfinden in die Parameter wissenschaftlichen Handelns adressiert den Kern akademischer Sozialisation. ${ }^{36}$

Die Hochschulwelt zu betreten und Teil des eigenen Lebens werden zu lassen, ist geprägt von Prozessen akademischer Sozialisation. ${ }^{37}$ Diese sind insbesondere dadurch gekennzeichnet, sich in die Selbstverständlichkeit wissenschaftlichen Denkens und Argumentierens, schriftlich $^{38}$ wie mündlich, einzufinden und das eigene Handeln davon leiten zu lassen. Brockbank \& McGill (1998) unterstreichen, wie weitreichend dieses Einfinden in das soziale System Hochschule für das (lernende und lehrende) Handeln aller Beteiligten ist. Sie schreiben:

„,...] we are deeply influenced by our experience, that learning contexts in higher education (as elsewhere) are themselves social constructs, that knowledge is socially constructed and that when learners enter and experience higher education they enter a system that is not value free and where power is exercised that can influence the process and learning of a student learner. The same applies to academic staff who have the responsibility, in part, to create the condition for learning to take place" (Brockbank \& McGill, 1998, S. 4).

Die Normen und Werte, die (angehende) Wissenschaftler*innen in ihrem Wirken leiten, sind somit ebenso wie die Art der Wissensproduktion begründet im jeweiligen sozialen

\footnotetext{
34 Vgl. Borst (2011, S. 21 ff.). Für bildungs- und erziehungswissenschaftliche Kontexte ist hervorzuheben, dass durch Beachtung der Sozialisation in der Analyse lernrelevanter Faktoren eine Fokusverschiebung stattfindet: Von pädagogischen auf gesellschaftliche Faktoren (Hurrelmann \& Bauer, 2015; Koller, 2017).

35 Basierend auf einem Modell des produktiv-realitätsverarbeitenden Subjekts beschreiben Hurrelmann \& Bauer (2015) hierzu, dass Sozialisation durch einen kontinuierlichen Abgleich innerer und äußerer Realität erfolgt. Das handelnde Subjekt ist hier im Zentrum.

36 Vgl. Huber (1991), der Wissenschaft als sozialen Prozess beschreibt, dessen Habitus man sich im Laufe des Studiums aneignet. Hochschulsozialisation definiert er (ebd., S. 419) als Aushandlungsprozess jener Dispositionen, die eine Person durch ihre Sozialisation in das Hochschulstudium mitbringt.

${ }^{37}$ Für einen Einblick sei auf das hierzu erschienen Themenheft der ZFHE, Vol 10(4), herausgegeben von Jenert et al. (2015) hingewiesen, zur Sozialisation in ein akademisches Fach zudem auf die Studien von Lindblom-Ylänne et al. (2006) und Trigwell et al. (2012).

38 Schmidt (2016) erläutert in ihrer Dissertation beispielsweise, inwiefern die akademische Sozialisation zu unterschiedlichem Sprachgebrauch im akademischen Schreiben führen kann. Dies begründet sie u.a. durch die identitätsbildende Kraft rollenspezifischen Sprachgebrauchs (ebd., S. 22f.).
} 
Kontext. Der Einfluss, den der soziale Kontext ${ }^{39}$ auf das Lernen hat, wird unter Berücksichtigung des Identitätsdiskurses unterstrichen. Wie verhält sich dieses Wissen nun zur Annahme, dass Identitäten nicht fix, sondern vielmehr fluide Konstrukte sind? Taylor (2008, S. 30) mutmaßt, dass diese ,[...] constructions are linked to the need for personal meaning.“ Diese Suche nach eigener Bedeutsamkeit ist hierbei verzahnt mit dem Bedürfnis, eine Situation oder Interaktion zu verstehen. Dieses Ergründen einer Information, das seinen Ursprung in der Begegnung eines Subjektes mit der Umwelt hat, offeriert eine weitere Verbindung des Konzepts der Identität mit dem des Lernens. Beide stehen in Relation zu Prozessen der Begegnung. Bereits in Kapitel 2.2 habe ich umrissen, dass derlei Begegnungsprozesse verschiedener Art sowie Grundlage lernrelevanter Reflexionsimpulse sein können. Ihnen inhärent ist, dass - mit Blick auf die Verschränkung von Identität und Lernen - derlei Begegnungen auch die Wahrnehmung eines Anderen in sich tragen können. Dass eine solche Begegnung mit dem Anderen Einfluss auf das Lernen auszuüben vermag, ergibt sich bei näherer Betrachtung des Identitätskonzeptes und seiner Grundannahme, dass Identität im Entstehen das konstruierte Andere braucht. Ein Anderes, das unter dem Begriff der Alterität und konzeptuell als Schwester der Identität verhandelt wird. Im Folgenden werde ich das Konzept der Alterität näher beleuchten und so die Verschränkung von Lernen, Identität und Alterität unterstreichen.

\subsection{Lernen und Alterität}

Mit Alterität wird jenes abstrakte Phänomen bezeichnet, durch das ein Subjekt ein Außerhalb erfährt und sich in Abgleich dazu verortet. In seiner Entstehung und insbesondere seinen philosophischen Wurzeln ist der Begriff der Alterität geprägt durch Wertungsfreiheit. Weder avisiert er, Fremdheit zu erzeugen, noch zu hierarchisieren. ${ }^{40}$ Vielmehr ist es der Wunsch, das Selbst besser zu verstehen, der sein Wirken motiviert.

In seinem Kern ist das Konzept der Alterität mit dem der Identität verbunden, da es den Mechanismus darstellt, über den ein Subjekt sich - in einer bestimmten sozialen Rolle gegenüber einem Anderen positioniert, das eigene Handeln (neu) verortet und so dem jeweiligen Selbst einen Sinn gibt. Alterität ermöglicht es, dieses Andere zu konstruieren und so den selbstvergewissernden Abgleich initial zu ermöglichen. Alterität ist dadurch Teil der fortwährenden Prozesse individueller Identitätskonstruktion. Die besondere Verbindung von Identität und Alterität wird beispielsweise durch die Arbeiten der französischen Philosophen Derrida und Deleuze ${ }^{41}$ hervorgehoben. Sie argumentieren, dass Identität und Alterität sich

\footnotetext{
39 Berger \& Luckmann weisen in ihrem Grundlagenwerk zum Sozialkonstruktivismus „The social construction of reality“ (1966) bereits auf den Einfluss des sozialen Kontextes auf alle Ebenen des Miteinanders sowie der Wissensproduktion und so auch auf das Lernen hin.

40 Terminologisch werden im philosophischen und kulturwissenschaftlichen Diskurs die Begriffe Alterität, Fremdheit und Alienität hinsichtlich ihres Grades der vom Subjekt wahrgenommenen Verschiedenheit voneinander unterschieden. Während Alterität wie hier beschrieben nicht wertend, sondern im Kern mit der Identität verbunden als Eins verstanden wird, zeichnen sich die beiden anderen Begriffe in ihrer Konnotation durch den Fokus auf Abgrenzung aus (vgl. u.a. Müller-Funk, 2016).

${ }^{41}$ Vgl. Müller-Funk (2016, S. 40; S. 221).
} 
nicht oppositionell gegenüberstehen, sondern sich vielmehr eine gemeinsame Grenze teilen. Diese Grenzverbindung prägt die Prozesse individueller Verortung, in der die Selbstannahme stets auch eine Fremdannahme ist.

Ausgehend von dieser Begriffs-Annäherung ergeben sich im Alteritätsdiskurs folgende Grundannahmen: Wenn Fremd- und Selbstannahme parallel erfolgen, kann das Verstehen des Anderen theoretische Rückschlüsse anbieten, um sich selbst als Person besser zu verstehen. ${ }^{42}$ Mehr noch: Im Prozess des Verstehens, so Bedorf (2011, S. 101), sei die „Andersheit der Anderen“ nicht bloß ,[...] beiläufige Zugabe, sondern der Anstoß, der diesen Prozess erst in Gang setzt und vielfältige Deutungsmöglichkeiten eröffnet.“ In seinem Hauptwerk „Das Sein und das Nichts "433 führt Sartre diesen Gedanken eine Stufe weiter. Er fokussiert darauf, dass, wenn es ein Mensch ist, den ich konstruiert als Anderen wahrnehme, ich im Spiegel dieser Begegnung selbst als Andere*r wahrgenommen werden kann. ${ }^{44}$ Durch den nunmehr bewussten Blick des Anderen auf mich selbst werde ich zum durch die Betrachtung konstruierten Subjekt, das durch ein Anderssein ${ }^{45}$ geprägt ist.

Wodurch eine solche Wahrnehmung des Anderen in Begegnungsprozessen geleitet werden kann, verdeutlicht sich in den Arbeiten Waldenfels'. ${ }^{46}$ Für ihn spiegelt sich die Wirkung, die von der Begegnung mit dem Anderen (bei ihm als das Fremde bezeichnet) ausgeht, im Prozess der Erfahrung eines Menschen wider. Er unterscheidet drei Modi dieser Erfahrung des Anderen: die Wahrnehmung des Andersseins der Anderen, des Andersseins des Systems sowie des Andersseins des Ich.

Durch das Wissen um die Verbindung von Selbst und dem Anderen eröffnet sich eine weitere Perspektive, um die Entstehung von Erfahrungen zu ergründen. Eine Erfahrung, die durch das Erleben von etwas Neuem, Überraschendem oder Unvorhergesehenen in das Bewusstsein einer Person dringt, kann durch die Begegnung mit dem wahrgenommenen Anderen erzeugt werden. Der Impuls, der hierbei generiert wird, um sich in der eigenen Rolle (sowie aufgrund eines neuen Wissens) zu verorten, kann so Folge der Begegnung mit dem Anderen sein. Verdeutlicht wird dies durch Rückbesinnung auf die Relevanz von Reflexionsprozessen, wie sie in Kapitel 2.3 skizziert wurde. Was ist es, das den Impuls zur Reflexion erzeugt? Ein evoziertes Innehalten im Prozess der Interaktion mit der Umwelt. Vor dem Hintergrund eines auf das Lernen bezogenen Alteritätsverständnisses wird dieses Innehalten $^{47}$ - ausgelöst durch Verwunderung, Unsicherheit oder Verwirrung - das ein

${ }^{42}$ Bredella (2010, S. xxiv) sagt: „Verstehen des Anderen hat Rückwirkungen auf das eigene Selbstverständnis. Es ist Teil eines Bildungsprozesses und besteht in der Veränderung des eigenen Vorverständnisses [...].“

${ }^{43}$ Erstmals erschienen 1943 in Paris, hier bearbeitet in der 2009 erschienen Ausgabe des Rowohlt Verlags.

44 Vgl. Sartre (2009, S. 415ff.) sowie Müller-Funk (2016, S. 66ff.).

45 An dieser Stelle sei darauf hingewiesen, dass in meiner Arbeit bewusst vom Anderssein gesprochen wird und nicht von Andersheit. Während Letztere die Konnotation der Starrheit in sich trägt, fokussiert erstere auch sprachlich darauf, ein subjektiv wahrgenommenes Phänomen zu beschreiben.

46 Vgl. hierzu u.a. Waldenfels (2013 [1990]), Müller-Funk (2016) und Koller (2012). Insbesondere Agostini (2016, S. 167) unterstreicht zudem, dass bei Waldenfels die Vorstellung eines responsiven Erfahrungsvollzugs vorherrscht, durch den er erfassen möchte, wodurch Phänomene der Erfahrung entstehen und wie sie strukturiert sind.

${ }^{47}$ Meyer-Drawe (2008, S. 15) charakterisiert ein solches Innehalten als Irritation, die zwar ,zeitraubend“, für das Lernen aber auch bedeutungsvoll sei. 
lernendes Subjekt als Impuls erfährt, in seiner möglichen Verbindung zur Begegnung mit dem Anderen sichtbar.

Es lässt sich festhalten, dass Prozessen der Identitätskonstruktion stets jene der Erschaffung des Anderen inhärent sind, dass letztere als Grundlage der Selbstvergewisserung und/oder Verortung als Subjekt dienen und dass sie gefasst im Konzept der Alterität verhandelt werden.

Alterität kann als Kern dessen verstanden werden, was die Begegnung mit dem Anderen und die darin ermöglichten Reflexionsimpulse in ihrer nicht-intendierten Qualität entstehen lässt: Impulse, die dem Selbst ermöglichen, im Nachvollziehen des Anderen etwas über das eigene Sein zu erkennen.

Übertragen auf das Lernen möchte ich zwei weitere Aspekte hervorheben: Auf Ebene der abstrakten Information ist die Auseinandersetzung mit dem Anderen im Feld der Bildungswissenschaften bereits implizit verhandelte Erkenntnis. Setzt doch der Akt des Verstehens einer Information voraus, dass sie mir zuvor als unbekannt und fremd begegnete. Verstehen und Lernen sind somit in sich mit der Begegnung mit dem Anderen verbunden. ${ }^{48}$ Weiter ist der dialogische Charakter im Prozess des Lernens, der zwischen neuer Information und vorhandenem Wissen wirkt, mit der Interdependenz von Identität und Alterität zu vergleichen. ${ }^{49}$

Die Verbindung des Konzeptes der Alterität mit Prozessen des Lernens ergibt sich bei genauerer Bertachtung beinahe mühelos. Sie kann als konzeptuelle Basis das Reservoir individuellen Wissens nutzen, über das eine Person ihre Identität sowie ihr Handeln begründet. Dies möchte ich kurz verdeutlichen. Das Andere, dem ich begegne, kann durch seine Verwobenheit mit meiner Identität, meinen Wissensstrukturen und Einstellungen nur ich selbst in dieser speziellen Ausprägung erfahren. Es sind meine Sinnkonstrukte und Wahrnehmungen sowie die Systeme, in denen ich mich als sozialisiertes Mitglied bewege, die das Andere für mich in dieser Form entstehen lassen. Im Rahmen meiner Arbeit an Teilstudie B habe ich diesen relevanten Aspekt mithilfe der Darstellung Sara Ahmeds (2000) wie folgt hervorgehoben:

"By experiencing something as different, as unfamiliar and strange, we find ourselves in the process of our own identity construction. As Ahmed (2000: 55) points out: "The strange is produced as a category within knowledge, rather than coming into being in an absence of knowledge." Hence, dealing with the otherness we experience offers us a gateway to encounter modes of our (individual) knowledge construction and invites us to challenge them" (Thielsch, 2019a, S. 11).

${ }^{48}$ Göhlich \& Zirfas (2007, S. 125) beschreiben „die Erfahrung eines Anderen, auf die das Subjekt eine Antwort finden muss“ als Grundsituation des Lernens.

${ }^{49}$ Bredella (2010, S. xx) fasst diesen dialogischen Charakter als intersubjektiven Aushandlungsprozess einer situativ erzeugten Information mit dem Vorverständnis einer Person sowie dem Prozess der Sinnbildung. 
Indem wir dem Anderen begegnen, eröffnet sich uns folglich eine Pforte, um die Beschaffenheit eigener Wissenskonstrukte zu durchdringen und ihren Wert für die eigene Identität und das, was wir wissen und lernen (wollen), zu reflektieren.

\subsection{Das Erkenntnisinteresse}

In ihrem Grundlagenwerk zum Lernen konstatieren Göhlich und Zirfas (2007, S. 180), dass der Gestaltung des Begegnens sowie der Auseinandersetzung mit dem Anderen - sei es ein Inhalt oder eine im Prozess beteiligte Person - „, höchste pädagogische Aufmerksamkeit zu gelten hat." Was sich für die Autoren aufgrund der sozialen Einbettung und des dialogischen Charakters von Lernprozessen ergibt, kann basierend auf den oben erläuterten Zusammenhängen von Reflexion, Identität und Alterität als Teil dieser Prozesse argumentativ bekräftigt werden. Die Begegnung mit dem Anderen ist Lern- und Lehrprozessen inhärent, ihre Relevanz durch das Wissen um die Beschaffenheit des Alteritätskonzeptes um eine weitere Perspektive begründet.

Die in den vorherigen Seiten argumentierte theoretische Fundierung hat verdeutlicht, welchen Mehrwert die Erforschung des Anderen im Lernen für bildungswissenschaftliche Diskurse haben kann. Was bislang impliziter oder expliziter in der Theorie verhandelt wurde, ${ }^{50}$ soll nun empirisch hergeleitet und als wissenschaftliches Instrumentarium eingeführt werden. Hierauf fußt das grundlegende Anliegen meiner Arbeit.

Alterität wird in meiner Forschung in der Verwobenheit mit ihrer Schwester, der Identität, genutzt, um besser zu verstehen, wann und wodurch im Lernen die Begegnung mit dem Anderen entstehen kann und welchen Mehrwert dies für individuelle Lernprozesse offeriert. Anhand der drei in meiner Dissertation durchgeführten empirischen Studien konnten Erkenntnisse über das analytische Potential des Konzeptes der Alterität zusammengetragen werden, um nunmehr jene Reflexionsimpulse zu benennen, die durch die Begegnung mit dem Anderen hervorgerufen werden. Diese Reflexionsimpulse können für das Handeln in akademischen Lehr- und Lernkontexten genutzt werden, um ihr Entstehen und ihr Wirken zu antizipieren und bewusst für die Gestaltung von Lehr- und/oder Lernkontexten heranzuziehen.

Das übergeordnete Ziel und somit Kern des Erkenntnisinteresses meiner Arbeit ist es, ein alteritätsbezogenes Lernverständnis zu begründen, das uns ermöglicht, Lernprozesse sowie ihre soziale Einbettung aus Perspektive der ihnen inhärenten Begegnungen mit dem Anderen nachzuvollziehen und für die Gestaltung und Analyse (akademischer) Bildungskontexte zu nutzen.

${ }^{50}$ Benner (1999) ebenso wie Schäfer (2004) und insbesondere Koller (2012) haben in der jüngeren Vergangenheit Verbindungslinien des philosophischen Diskurses über das Andere bzw. Alterität mit den Traditionen der Erziehungswissenschaft verhandelt und/oder auf das Konzept der Bildung angewandt. 
Mithilfe der von Waldenfels benannten Wahrnehmungsebenen der Begegnung mit dem Anderen - durch das Anderssein des Ich, des Anderen, des Systems - sowie der durch Schloos für das Lernen benannten Dimension des Andersseins des Themas, wird in der Auswertung der drei hier entstandenen Studien eine Systematik der Analyse lerninhärenter Begegnungsprozesse genutzt, um im Sinne des Erkenntnisinteresses meiner Forschung einen Ansatz zur Anwendung des Alteritätskonzeptes auf akademisches Lernen vorzustellen.

Die einzelnen, für diese Forschung durchgeführten Studien, haben sich zum einen am oben genannten Erkenntnisinteresse orientiert. Zum anderen haben sie neben ihren individuellen Forschungsfragen Informationen zu zwei Leitfragen gesammelt.

\section{Leitfrage 1 Wodurch kann die Begegnung mit dem Anderen in einem gegebenen} akademischen Lehr-Lernkontext entstehen?

\section{Leitfrage 2 Welche Erkenntnisse über den jeweiligen Lernprozess können aufgrund} des Wissens um das Konzept der Alterität gewonnen werden?

In Verbindung zur antizipierten Anwendung des Alteritätskonzepts auf das Lernen stellen diese Leitfragen eine Brücke zum übergeordneten Ziel des Dissertationsprojektes dar. Das folgende Kapitel stellt in komprimierter Form die drei für dieses Vorhaben durchgeführten Studien vor und gibt in Kapitel 3.4 schließlich einen Einblick in die gesammelten Informationen hinsichtlich der beiden Leitfragen. So untermauert, erfolgt in Kapitel 4 die Analyse des Mehrwerts, den die Perspektiven des Alteritätskonzepts auf Prozesse des Lernens ermöglicht. Vollzogen wird sie mithilfe des hierfür entwickelten Vier-Ebenen-Modells der Wahrnehmung des Andersseins.

\section{Teile des Ganzen - Was einzeln erforscht wurde}

Jeder der drei Teilstudien ${ }^{51}$ wohnt eine hochschuldidaktische Perspektive sowie eine anwendungsbezogene Zielsetzung inne. Ausgerichtet am Erkenntnisinteresse sollte in erster Linie neues Wissen über alteritätsbezogene Begegnungsprozesse generiert werden. Zudem wurde in der Durchführung jeder Studie darauf geachtet, dieses Wissen nach Möglichkeit direkt anzuwenden und so sein Potential zur Unterstützung hochschulischen Lehrens und Lernens zu erproben bzw. zu unterstreichen. ${ }^{52}$

\footnotetext{
51 Vgl. Kapitel 9 und die hier gegebenen Kurzvorstellungen der Teilstudien.

52 Die Motivation, in der Gestaltung meiner Studien den Anwendungsbezug stets mitzudenken, verdeutlicht sich in Anlehnung an eine Aussage Fleischmanns (2019), die er im Rahmen einer Keynote im Februar 2019 formulierte: Die Erkenntnisse der Lehr-Lernforschung, so Fleischmann, stellen in erster Linie Information zur theoretischen Einschätzung eines lernbezogenen Phänomens dar. Sie heben hervor, was Lernen begünstigt oder behindert und formulieren erste Implikationen für die Praxis. Der Übertrag in einen konkreten Lehr-Lernkontext liegt bei den Lehrenden... und stellt für sie oft eine Hürde dar. Dieser Herausforderung, die ich als Hochschuldidaktikerin selbst
} 
In diesem Kapitel wird jede der drei Teilstudien in Kürze präsentiert. Der Fokus liegt hierbei auf der komprimierten Vorstellung des jeweiligen Forschungsdesigns sowie der ermittelten Ergebnisse. Die oben beleuchteten Konzepte der Sozialisation, Erfahrung, Identität und Alterität stehen in ihrer Verwobenheit - z.T. implizit, z.T. explizit in der jeweiligen Arbeit behandelt - im Zentrum jedes Teilprojekts. Weiter nimmt das Konzept der Reflexion eine Sonderrolle ein, da es in allen Fällen nicht nur methodisches Instrument des Zugangs war, sondern gleichsam als konstituierendes Moment jener Wirkungsmechanismen der Begegnung in die Argumentationen der Kurzvorstellungen eingebunden ist.

Die Teilstudien werden hier mithilfe der ihnen zugrundeliegenden Logik in Relation zum Gesamtprojekt vorgestellt. In ihrer Abfolge und Argumentation bauen sie im Sinne einer kritisch-konstruktiven Didaktik ${ }^{53}$ sowie mit Blick auf die übergeordnete Forschungsfrage aufeinander auf und wandeln ihren Zugang vom induktiven Ermitteln eines Phänomens zum deduktiven Überprüfen desselben. Während in der ersten Studie ein Ausgangspunkt geschaffen und das Offensichtliche beleuchtet wird, fokussiert die zweite Arbeit darauf, das mit Blick auf mein Erkenntnisinteresse nunmehr Antizipierte zu ergründen. Inhalt und Setting dieser zweiten Arbeit motivieren den Zugang zur finalen und dritten Studie, in der das konkret Erwartete schlussendlich überprüft wird. Was und wie genau geforscht wurde, ist Inhalt der nächsten Seiten.

\subsection{Das Offensichtliche beleuchten: Teilstudie A}

Veröffentlicht in der Zeitschrift für Hochschulentwicklung in 2017 hat das erste Teilprojekt sich mit den Herausforderungen befasst, die im Zuge internationaler Hochschulstrukturen von den beteiligten Studierenden und Lehrenden erfahren werden. Das Paper mit dem Titel Approaching the Invisible: Hidden Curriculum and Implicit Expectations in Higher Education fasst die Vorgehensweisen und Erkenntnisse dieser Forschung zusammen. Methodologisch verortet als Arbeit der kritischen Diskursanalyse ${ }^{54}$ ist es ihr Ziel, die soziale Realität eines Diskurses nicht nur zu erfassen, sondern auch auf sie einzuwirken. Ausgehend davon, dass ein Diskurs Ursprung jener sozialen Phänomene ist, die er braucht, um sich selbst zu legitimieren, zielt kritische Diskursanalyse darauf ab, den im Diskurs verhafteten Individuen, die von ihm geleiteten sind und ihn gleichzeitig reproduzieren, Reflexionsimpulse zu offerieren, um die diskursive Wirkungsmacht zu erkennen und zu durchbrechen. Die Normen und Werte einer gleich sozialisierten Gruppe werden durch einen solchen Diskurs geprägt. In ihrer verborgenen und so impliziten Anwendung sind sie es, die auch das Handeln in einem bestimmten akademischen Kontext beeinflussen.

immer wieder wahrnehme, sollte in der hier entstandenen Forschung durch Verzahnung mit der Praxis entgegengewirkt werden.

53 Klafki (2007, S. 98 ff.) erläutert hierzu, dass gemäß einer kritisch-konstruktiven Didaktik hermeneutische, empirische und gesellschaftskritische Forschungszugänge in Kombination zum Einsatz kommen soll(t)en, um den Prozess der Erkenntnisgewinnung zu bereichern.

54 Vgl. u.a. Fairclough (2003) und Keller (2011). 
Die Arbeit in diesem Projekt fußt auf den Erkenntnissen der Internationalisierung der Hochschule im Allgemeinen und denen zur Existenz des verborgenen Lehrplans oder bidden curriculum im Besonderen. Subsumierbar in den Grundannahmen, dass der Begriff Curriculum alles umfasst, das die Lernerfahrung von Studierenden beeinflusst (Carroll, 2015, S. 105) und dass ein Curriculum sich aus verschiedenen, ineinander verwobenen Elementen zusammensetzt (Leask, 2015), wurde in den letzten Jahren der Internationalisierung an Hochschulen Aufmerksamkeit geschenkt. Der Gedanke, dass es an jeder Hochschule ein bidden curriculum gibt, das insbesondere internationale Studierende in ihrem Lernen vor Herausforderungen stellt, wird im Rahmen dieser Bestrebungen verdeutlicht; Wege, um dasselbe zu ergründen, werden jedoch kaum thematisiert. Vielmehr wird der Umgang damit als erneuter Sozialisationsprozess verhandelt, der von internationalen Studierenden durchlaufen werden müsse, um die sozialen Rituale (vgl. Killick, 2015) zu durchdringen, die im jeweiligen akademischen Kontext das Lehren und Lernen rahmen. Doch was, wenn der Aufenthalt zu kurz ist, um einen solch komplexen Prozess wie den der Sozialisation zu beschreiten? Im Fall von Austauschstudierenden, so die hier verwendete Grundannahme, ist dies der Fall. Mit dem Ziel, Bereiche des wahrgenommenen bidden curriculum zu ermitteln und infolgedessen ein Werkzeug zu entwickeln, das Lehrenden hilft, der Verunsicherung des Verborgenen entgegenzuwirken, wurde die Forschung dieses Teilprojektes designed.

Basierend auf dem Wissen um die Prozesselemente des Lernens - abstrakte Information, konkrete Erfahrung und verbindende Reflexion - wurden Aussagen zu Erfahrungen von Austauschstudierenden mit der erlebten Lehr- und Lernpraxis an einer Zieluniversität erhoben. Die Stichprobe setzte sich aus 480 Individuen zusammen, die im Jahr 2016 eine Zeit als Austauschstudierende an der Universität Göttingen verbracht haben. Ein Fragebogen ${ }^{55}$ mit acht Items zu Lernbiographie-relevanten Aspekten und drei offenen Fragen wurde den Individuen der Stichprobe elektronisch zugänglich gemacht. Die offenen Fragen wurden mit Fokus darauf entwickelt, durch drei unterschiedliche Reflexionsimpulse das Erlebte im Auslandssemester in Erinnerung zu rufen: den Vergleich zwischen Erlebtem und Vertrautem, das Beschreiben einer Herausforderung und das Formulieren eines Rates an zukünftige Studierende. Die Daten aus diesem Bereich der Erhebung wurden inhaltsanalytisch ausgewertet und hierzu das Vorgehen der induktiven Kategorienbildung ${ }^{56}$ genutzt. Klassifiziert in die zwei übergeordneten Bereiche des Staying abroad und Studying abroad konnten die Inhalte in den Daten drei Kategorien zugeordnet werden: der Relevanz von Sprachkenntnissen, den individuellen Bedürfnissen jenseits akademischer Kontexte sowie den Spuren individueller akademischer Sozialisation. Die zuletzt genannte Unterkategorie und ihre jeweiligen Codes wurden als Grundlage genutzt, um einen Reflexionsbogen für Lehrende zu entwickeln. Mit der Intention zu überprüfen, ob die hier entstandenen Reflexionsfragen alle zuvor ermittelten Kategorien des Studying abroad avisieren könnten, wurde eine kleine Gruppe Lehrender eingeladen, den Bogen zu testen. Die hierbei gesammelten Daten wurden, in diesem Fall

${ }_{55}$ Der Fragebogen kann bei Interesse per Email bei der Autorin angefragt werden.

56 Vgl. Kuckartz (2016) und Mayring (2014). 
mittels deduktiver Kategorienbildung, bezüglich ihrer intendierten Zielrichtung ausgewertet und konnten die gewünschte Reflexionsbreite bestätigen. Als Instrument zur Reflexion eigener Erwartungen an akademisches Lehren und Lernen kann der Bogen somit helfen, jenen Herausforderungen vorzubeugen, die eine Begegnung mit impliziten Handlungsroutinen und den dahinterliegenden Regeln unterschiedlicher Diskurse akademischer Sozialisation mit sich bringen können.

Ausgehend vom Offensichtlichen, dem in unterschiedlichen Studien angeführten Begegnen eines anderen, verborgenen Lehrplans, hat dieses Teilprojekt näher beleuchtet, aufgrund welcher qualitativen Aspekte die Begegnung mit dem Anderen im Auslandssemester erfahren werden können und so den Prozess der akademischen Sozialisation als begründendes Element unterstrichen. Im nächsten Schritt meiner Forschung sollte daran anschließend das Handeln der Lehrenden näher ergründet werden. Zuvor möchte ich die gewonnenen Erkenntnisse über das Phänomen des bidden curriculum mit einem Zitat aus dem hier entstandenen Paper abschließen, das meines Erachtens gleichermaßen zusammenfassend als auch diese Überleitung gestaltend ist:

"To determine, where a hidden curriculum ends and where a person's individual values and expectations begin, seems to be impossible. Yet, academic teaching staff can lessen the power of such implicit expectations in becoming more transparent in their own teaching. Within this flexible system of a hidden curriculum, they are located at the core of discursive practices, why addressing them and their teaching beliefs constitute a sensible starting point to approach the invisible" (Thielsch, 2017, S. 185).

\subsection{Das Antizipierte ergründen: Teilstudie B}

Im Vergleich zu den beiden anderen Arbeiten, die im Rahmen dieser Dissertation durchgeführt wurden, zeichnet sich diese durch ein besonderes Maß an Interdisziplinarität aus; sowohl auf inhaltlicher als auch auf methodischer Ebene. Bildungswissenschaftlichhochschuldidaktische Perspektiven wurden im geisteswissenschaftlich-musikethnologischen Kontext angewandt, um den sozialwissenschaftlich-politisch begründeten Ansatz des Postkolonialismus im Lehrkontext durch ethnographische Methoden zu beforschen. Vor diesem Hintergrund fiel die Wahl eines passenden Publikationsorgans zunächst schwer. Unter dem Titel Listening Out and Dealing with Otherness. A Postcolonial Approach to Higher Education Teaching sind die Ergebnisse des Projektes im internationalen Sage Journal Arts and Humanities in Higher Education zu Beginn 2019 erschienen. ${ }^{57}$

Die Forschung dieser Teilstudie setzt ausgehend vom Wissen um den Einfluss akademischer Sozialisation auf die Gestaltung hochschulischen Lehrens und Lernens und ihrer diskursiven Praktiken an und ergründet im Sinne der Dekolonisierung der Hochschulen die (Re)Produktion hegemonialer Perspektiven durch Prozesse der Wissensproduktion im Kontext akademischer Lehre. Welche Herausforderungen und Chancen würden sich ergeben,

${ }^{57}$ In diesem Journal wurde 2016 die für mein Paper richtungsweisende Abhandlung Mbembes zur Dekolonisierung der Universitäten veröffentlicht und eine Anbindung meines Papers somit offenbar. 
eine Lehrveranstaltung im Sinne des Postkolonialismus zu designen und so bewusst Situationen zu gestalten, in denen sich Studierende, aber auch Lehrende in kontinuierlicher Auseinandersetzung mit dem Anderen wiederfinden würden? Welchen Mehrwert - abgesehen vom Vorantreiben akademischer Dekolonisierung - würde ein solches Lehrhandeln mit Blick auf das Lernen haben? Zur Beantwortung dieser Forschungsfragen bot sich ein auf Involviertheit basierendes Eintauchen in das Untersuchungsfeld an. Entwickelt und realisiert als Feldforschungsprojekt orientierte sich mein Vorgehen in dieser Teilstudie an der von Culhane (2016) formulierten Grundannahme ethnographischen Handelns: Als Bündel von Erhebungsmethoden ermöglicht Feldforschung nicht durch Beobachtung alleine, sondern insgesamt durch Interaktion mit dem Forschungsfeld, Daten zu sammeln und Wissen zu generieren. Jede ethnographische Wissensproduktion sei ein Aushandlungsprozess zwischen Individuen sowie der vorausgehenden Gestaltung des In-Kontakt-Tretens mit dem Feld. Methodologisch zielt Feldforschung heute darauf ab, den Prozess der Erkenntnisgewinnung kritisch zu beleuchten ${ }^{58}$ und als Forscher*in immer wieder innezuhalten und danach zu fragen, wodurch man selbst weiß, was man zu wissen glaubt. In der hier durchgeführten Studie kamen dies berücksichtigend als Methoden die Teilnehmende Beobachtung, die damit verknüpften, leitfragengestützten Feldnotizen ${ }^{59}$ sowie eine schriftliche Befragung der Beteiligten am Ende des Projektes zum Einsatz. Die triangulierte Auswertung der gesammelten Daten erfolgte in Form der inhaltlich-interpretierenden Analyse.

Gemeinsam mit einer Fachwissenschaftlerin wurde als Teaching Team eine Lehrveranstaltung für den Masterstudiengang Musikwissenschaften entwickelt, die sowohl inhaltlich als auch methodisch eine nicht-normative Annäherung an das musikalische Genre der Hindustani Klassischen Musik ermöglichen sollte. Im Seminar wurde sich dem Genre gemeinsam mit einer kleinen Gruppe Studierender durch Hören und Beschreiben angenähert, die Abhandlungen westlich sozialisierter Forscher*innen sowie Vergleiche mit vertraute(re)n Genres oder sonstiger, in diesem Kontext verwendeter Klassifikationsschemata hingegen wurden ausgespart. Dieser, mit den Studierenden abgestimmte postkoloniale Modus des Lehrens und Lernens, fußte sowohl auf den Arbeiten postkolonialer Theoretiker*innen ${ }^{60}$ als auch auf Werken zur kritischen Reflexion des Bildungsbegriffes ${ }^{61}$, der Individualität des Lernens sowie der akademischen Sozialisation. Handlungsleitend für den Einsatz dieses bislang nicht erforschten Lehransatzes wurde die von Young (2003, S. 7) formulierte

\footnotetext{
${ }^{58}$ Eine kritische Haltung ist allen Schritten im ethnographischen Forschungsprozess innewohnend. Die durch den Sammelband von Clifford \& Marcus (1986) angestoßene Debatte, dass ethnographisches Schreiben Ergebnis verschiedenster Interpretationsprozesse sei, erlangte durch die Arbeiten von Geertz (u.a. 2008) Bekanntheit und prägt seither die kritisch reflexive Auseinandersetzung ethnologischer Forschung.

${ }^{59}$ Die Leitfragen können bei Interesse per Email bei der Autorin angefragt werden.

${ }^{60}$ Insbesondere die Arbeiten Bhabhas „The Location of Culture“ (2012 [1994]), Saids „Orientalism“ (2003 [1978]) und Spivaks „Can the subaltern speak“" (1994) sind hier zu nennen.

${ }_{61}$ Vgl. Klafki (2007) sowie Taylor (2017).
} 
Postkolonialismus-Definition $^{62}$ genutzt und daran anschließend der Kern postkolonialer Pädagogik erörtert. Konkret lautet es im hierzu erschienen Artikel wie folgt:

"Postcolonial pedagogy seeks to intervene with given epistemologies, it seeks to open discourses of knowledge production in the classroom and beyond. It seeks to change the way people think about academia and its supposedly objective nature and thus invites academics and students to change their perceptions and behaviours in teaching and learning contexts. It disturbs the asymmetrical order of the classroom by threatening power relations based on notions of right and wrong and thereby challenges the reproduction of normative thinking. In doing so, it refuses to value one way of knowledge (production) above another" (Thielsch, 2019a, S. 13f.).

Die hier durchgeführte Feldforschung hat neben dieser Definition postkolonialer Lehre mehrere Ergebnisse mit Blick auf den erfolgreichen Einsatz eines solchen Lehransatzes in der Universität hervorgebracht. Durch gezielte Gestaltung der Begegnung des Anderen (Dealing with otherness) und einem Fokus auf die Individualität im Lernen sowie der Wissensproduktion (Listening out) kann den entstehenden Herausforderungen im Lehren begegnet werden. In der planerisch antizipierten Begegnung des Unbekannten wird das akademische Selbst der Lernenden (und Lehrenden) herausgefordert. Die erwarteten Prozesse der Identitäts(Re)Konstruktion konnten als Anlass genommen werden, dem erlebten Anderen durch Thematisierung des Wie im Seminar zu begegnen und so die individuelle Produktion neuen Wissens zu hinterfragen. Das Potential eines postkolonialen Lehransatzes war so im Seminargeschehen unmittelbar, aber auch im Vor- und Nachbereiten der Lehre als Teaching Team erfahrbar. Durch die Auseinandersetzung mit den Wahrnehmungen des anderen Team Teaching-Mitglieds wurden Impulse erzeugt, um sich der eigenen Verwobenheit mit den Normen westlicher Academia kontinuierlich bewusst zu werden, das eigene Lehrverständnis zu reflektieren und anhand der Perspektiven des anderen Team Teaching-Mitglieds die eigene Wahrnehmung des Lehrgeschehens anzureichern. In der gemeinsamen Lehre - so wurde in dieser Studie verdeutlicht - liegt die Chance, dem Anderen zu begegnen und die hier gewonnenen Impulse im Sinne der Lernförderung ${ }^{63} \mathrm{zu}$ nutzen. Eine Annahme, die es im dritten Teilprojekt meiner Forschung zu überprüfen galt.

\subsection{Das Erwartete überprüfen: Teilstudie C}

Geleitet von der Erkenntnis, dass die Begegnung mit dem Anderen in Lehr- und Lernsituationen jene Reflexionsimpulse zu erzeugen vermag, die dabei helfen, eigene Handlungsroutinen und Erwartungen zu ergründen, wurde das letzte Teilprojekt im Rahmen der Dissertation entwickelt. Das hierfür gewählte Forschungsfeld - die als Teaching Team realisierten Lehre - hatte sich hierfür in der Vergangenheit auf zwei Ebenen als sinnvoll

62 " $[\mathrm{P}]$ ostcolonialism seeks to intervene, to force its alternative knowledges into the power structures of the west as well as the non-west. It seeks to change the way people think, the way they behave [...]. It disturbs the order of the world. It threatens privilege and power. It refuses the superiority of western cultures" (Young, 2003, S. 7).

${ }^{63}$ Sowohl hinsichtlich des Lernens der Studierenden als auch bezogen auf den Prozess der eigenen Weiterbildung als Lehrperson. 
angekündigt: zum einen durch die oben skizzierten Erkenntnisse im Einsatz postkolonialer Lehre, zum anderen aufgrund der Daten einer ersten Analyse wahrgenommener Team Teaching-Realitäten im Zeitraum 2011-2014 (Thielsch, 2016). In letzterer wurden Aussagen von Team Teaching-Lehrenden zu Motivation und Gestaltung der gemeinsamen Lehre zusammengetragen sowie inhaltsanalytisch untersucht und ein potentieller Mehrwert für die Weiterentwicklung individueller Lehrkompetenzen ermittelt. Dieser Mehrwert sollte anhand der im Rahmen des Dissertationsprojektes gewonnenen Informationen zum Potential der Begegnung mit dem Anderen sowie der Möglichkeit, dieses unter Verwendung des Alteritätskonzeptes zu explizieren, nunmehr statistisch untersucht werden.

Erschienen in der durch die Vielfalt der hochschuldidaktischen Community Deutschlands geprägten Zeitschrift die hochschullehre stellt der hierzu entstandene Artikel Feedback in Team Teaching-Konstellationen. Eine Studie zur Lebrkompetenzentwicklung durch Begegnung anderer Wissensformen das Vorgehen und die Erkenntnisse dieser Forschung dar. Das hier leitende Erkenntnisinteresse - inwiefern durch die Lehre als Teaching Team Feedbackinformationen ermöglicht werden, die den beteiligten Lehrenden Anlass zur (Weiter-) Entwicklung der eigenen Lehrkompetenzen geben - fußt in seiner Argumentation auf drei theoretischen Grundlagen: Informationen zum Prozess der Lehrkompetenzentwicklung einer Person, der Lehre als Teaching Team in akademischen Kontexten sowie der Relevanz von Feedbackprozessen im individuellen Lernen. ${ }^{64}$ Mithilfe eines neu entwickelten Instruments zur Erfassung des Prozesses der Lehrkompetenzentwicklung in Hochschulen wurde ein Fragebogen konzipiert, der zum einen die Realität der jeweiligen Team Teaching-Erfahrung erfragt und zum anderen das von den Lehrenden hierbei wahrgenommenen Feedback. Die statistische Auswertung der über einen Online-Fragebogen ${ }^{65}$ gewonnenen Daten ehemaliger Team Teaching-Lehrender hat ergeben, dass die Lehre in dieser Konstellation nicht nur die avisierte Lehrkompetenzentwicklung durch die in ihr ermöglichten Feedbackinformationen fördert, sondern dass diese Informationen ihren Wert auch durch die Begegnung mit dem Anderen erhalten. Inhaltlich ausgewertet anhand der drei Team Teaching-inhärenten Feedbackquellen - des Team Teaching-Mitglieds, des Selbst sowie der Reaktion der Studierenden - kann anhand der gewonnenen Daten von 24 erfahrenen Lehrenden und 46 Lehreinsteiger*innen argumentiert werden, wodurch und wie ausgeprägt Lehrende mit unterschiedlicher Lehrerfahrung Feedbackinformationen wahrnehmen. Im zu diesem Projekt entstandenen Artikel (Thielsch, 2019b, S. 181) werden die Erkenntnisse wie folgt zusammengefasst:

„Die Lehre in Team Teaching-Konstellationen ermöglicht Feedbackinformationen durch

1. das Explizieren eigener Sinnlogiken sowie Wahrnehmungen im Teaching Team,

2. das Erfahren unterschiedlicher Lehrstile und -ansätze sowie ihrer Wirkung auf die Studierenden,

${ }^{64}$ Vgl. u.a. Hattie \& Timperley (2007), Lusk et al. (2016) sowie Trautwein \& Merkt (2013).

${ }^{65}$ Der Fragebogen kann bei Interesse per Email bei der Autorin angefragt werden. 
3. das Erkennen anderer Wahrnehmungen und Deutungsmuster sowie den komplementär verlaufenden Abgleich mit eigenen Wissensstrukturen und ihren Hintergründen,

4. das Erkennen eigener Erwartungen und Ziele als Lehrperson in der Wissenschaft.“

Obgleich die Lehrerfahrung die Wahrnehmung der jeweiligen Gruppe zu beeinflussen scheint, zeichnet sich hinsichtlich der Alteritäts-bezogenen Items (auf einer Viererskale von 1 = trifft nicht $q u$ bis $4=$ trifft $q u$ ) bei der gesamten Stichprobe die Tendenz ab, dem Anderen nicht nur begegnet zu sein, sondern daraus eine Rückmeldung für das eigene Lehrhandeln wahrgenommen zu haben. Beispielsweise Impulse, die einen Einfluss auf die Wahrnehmung des eigenen Lehrstils, die Erwartungen an Studierende oder die methodische Gestaltung der eigenen Lehre haben konnten, wie die ausgewählten Daten in Tabelle 1 vermuten lassen.

\begin{tabular}{|l|c|c|c|}
\hline Item & $\begin{array}{c}\text { Einsteiger*in } \\
\mathrm{M}(\mathrm{SD})\end{array}$ & $\begin{array}{c}\text { Erfahrene*r } \\
\mathrm{M}(\mathrm{SD})\end{array}$ & $\begin{array}{c}\text { Gesamt } \\
\mathrm{M}(\mathrm{SD})\end{array}$ \\
\hline $\begin{array}{l}\text { Bei der Vor- und Nachbereitung des gemeinsamen Lehrens wurde mir } \\
\text { deutlich, was mir selbst in der Lehre wichtig ist. }\end{array}$ & $3,72(, 50)$ & $3,08(1,02)$ & $3,5(, 78)$ \\
\hline $\begin{array}{l}\text { Während des Lehrens habe ich erlebt, wie Studierende auf verschiedene } \\
\text { Lehrstile reagieren. }\end{array}$ & $2,87(1,17)$ & $3,04(1,08)$ & $2,93(1,13)$ \\
\hline $\begin{array}{l}\text { Mein* Team Teaching-Partner*in hat mein geplantes Lehrhandeln } \\
\text { kritisch hinterfragt, so dass ich es überdenken konnte. }\end{array}$ & $2,83(1,00)$ & $2,54(1,02)$ & $2,73(1,01)$ \\
\hline $\begin{array}{l}\text { Bei der Vor- und Nachbereitung des gemeinsamen Lehrens haben wir } \\
\text { Situationen reflektiert, in denen das Verhalten der Studierenden uns } \\
\text { verwundert haben. }\end{array}$ & $2,67(1,30)$ & $3,38(, 92)$ & $2,91(1,22)$ \\
\hline
\end{tabular}

Tab. 1: Wahrgenommene Begegnung mit dem Anderen in der Lehre als Teaching Team $(\mathrm{n}=70)$

Im Rahmen dieses Teilprojektes konnte so anhand der gewonnenen Daten die Annahme bestärkt werden, dass in akademischen Lehr- und Lernkontexten die Begegnung mit dem Anderen in verschiedenen Ausprägungen möglich ist und in der Lehre als Teaching Team auch erfahren wurde. Die in diesen Begegnungen erzeugten Reflexionsimpulse adressieren auf die eine oder andere Weise die Identität der Lehrperson. Ihr Wissen um die Gestaltung der Lehre sowie um die dahinterliegenden Werte konnte in Reibung mit und Abgleich zu den Perspektiven des Team Teaching-Mitglieds durch die Wirkungsmacht des Konzeptes der Alterität neu verhandelt und erweitert werden. Die Weiterentwicklung der eigenen Lehrkompetenzen konnte dabei adressiert werden, ein Lernen über das eigene Handeln als Lehrperson war möglich.

\subsection{Zwischenfazit}

Alle drei Studien haben hinsichtlich der in Kapitel 2.6 vorgestellten Leitfragen und trotz ihrer inhaltlichen, methodischen und kontextuellen Unterschiede Erkenntnisse für das Ziel des Gesamtprojektes ergeben. Gebündelt erlauben sie ein erstes Zwischenfazit.

Zunächst wird hier eine Kurzzusammenfassung jener Punkte gegeben, die mit Blick auf die erste Leitfrage als Aspekte und Kontexte ermittelt werden konnten, in denen die 
Begegnung mit dem Anderen im Rahmen der drei Studien - in ihrer oben eingeführten Reihenfolge - hervorgerufen wurde.

\section{Leitfrage 1 Wodurch kann die Begegnung mit dem Anderen in einem gegebenen akademischen Lehr-Lernkontext entstehen?}

Teilstudie A Approaching the Invisible

○ durch nicht explizierte Erwartungen hinsichtlich der Interaktion in Lehrveranstaltungen

○ durch nicht explizierte Erwartungen an studentische Lernhandlungen

- durch unbekannte Lehrformate und ihre Regeln

- durch unerwartete Vorgaben oder Freiräume in der Gestaltung des Lernens

○ durch Missverständnisse mit verbalen und non-verbalen Auslösern

○ durch unbekannte Modalitäten des Prüfungssystems

Teilstudie B Listening Out and Dealing with Otherness

○ durch den Widerspruch geforderter/erwarteter Handlungen im Seminar

○ durch unerwartete Rollenverteilungen \& Hierarchien zwischen Studierenden und Lehrenden

O durch Erleben entgegengesetzter Erwartungen in anderen Seminaren

- durch Kennenlernen der Lernwege Anderer sowie Explizieren der eigenen Lernhandlungen

○ durch Erkennen unterschiedlicher Wahrnehmungen der studentischen Gruppe und ihrer Bedarfe im Teaching Team

- durch unterschiedliche Wahrnehmungen zur Gestaltung des Lehrens und Lernens im Teaching Team

Teilstudie C Feedback in Team Teaching-Konstellationen

○ durch den Austausch über individuelle Erwartungen in der Lehre

○ durch die Reaktion der Studierenden auf versch. Lehrestile \& Methoden

○ durch Einschätzung und Wahrnehmung des Team Teaching-Mitglieds

$\circ$ durch Beobachtung der Lehrhandlungen des Team TeachingMitglieds

- durch den Prozess der Selbstreflexion und der Erkenntnis, auf bestimmte Lehrhandlungen bewusst (weiter) zu verzichten oder sie zukünftig zu integrieren

Während diese Punkte mit Blick auf die Bearbeitung des Gesamtprojektes relevant sind und insbesondere die Nachvollziehbarkeit der weiteren Argumentation unterfüttern, sind es 
die Erkenntnisse und Informationen in Relation zu Leitfrage 2, die ankündigen, was Grundlage des entwickelten Modells zur Anwendung des alteritätsbezogenen Lernverständnisses sein konnte.

\section{Leitfrage 2 Welche Erkenntnisse über den jeweiligen Lernprozess können aufgrund des Wissens um das Konzept der Alterität gewonnen werden?}

Teilstudie A Approaching the invisible

Das Wissen um das Konzept der Alterität ermöglicht hier, auf Basis widersprüchlicher diskursiver Praktiken die eigene Involviertheit in ihrer (Re)Produktion zu erkennen. Durch gezielte Reflexion der Bereiche des von den Studierenden wahrgenommenen bidden curriculum können Wissenschaftler*innen die eigenen Erwartungen im Lehren und Lernen in ihrer Disziplin ergründen und in der Lehre explizieren.

Teilstudie B Listening out and dealing with otherness

Durch Perspektive der Alterität können die antizipierten sowie neu erfahrenen Herausforderungen in der Anwendung eines postkolonialen Lehrzugangs erkannt und abgemildert werden. Insbesondere durch den Austausch mit Anderen über das eigene Lernen (als Studierende*r) und Lehren (im Teaching Team) können die Logik individueller Handlungen sowie die eigene Involviertheit in ein bestimmtes System der Wissenskonstruktion bewusst(er) wahrgenommen werden.

Teilstudie C Feedback in Team Teaching-Konstellationen

Der Fokus auf Alterität hilft dabei, die vielschichtigen Feedbackinformationen, denen Team Teaching-Mitglieder im gemeinsamen Lehren begegnen können, zu erfassen und die durch sie ausgelösten Momente des Innehaltens, Umdenkens oder Versicherns in ihren Ausprägungen zu analysieren. Durch die Begegnung der Lehransätze und Sinnstrukturen des Team Teaching-Mitglieds sowie der Reaktion der Studierenden darauf, können Impulse zur Weiterentwicklung der eigenen Lehrkompetenzen erzeugt werden.

Für sich beinhalten alle drei vorgestellten Studien Hinweise, wodurch die Anwendung des Konzeptes der Alterität neue Einblicke auf akademisches Lehren und Lernen ermöglicht. Um diese Einblicke für eine breitere Verwendung nutzen zu können, werden sie im nächsten Kapitel systematisiert. Das Modell, das hierfür verwendet wird und das in der Bearbeitung dieses Forschungsprojektes entstanden ist, stelle ich im nun folgenden Kapitel und Herzstück meiner Arbeit vor. 


\section{Lernen und das Andere - Was hier entstanden ist}

Wir wissen, dass die Entstehung von Identitäten ein fortlaufender Prozess ist, der durch die Aushandlung und Konsolidierung, Abgrenzung und Selbstvergewisserung gekennzeichnet ist und einem Subjekt erlaubt, sich in einem sozialen Kontext bewusst zu verorten. Wir wissen weiter, dass die Begegnung mit anderen Perspektiven, Handlungslogiken und Werten, die gefasst im Konzept der Alterität - den Auslöser für eine solche Verortung darstellen kann und so die notwendige Reibungsfläche evoziert, um sich der eigenen Positionierung zu vergewissern. Ein Mehrwert, der für die Gestaltung akademischer Lehr- und Lernkontexte genutzt werden kann und als Ausgangspunkt eines alteritätsbezogenen Lernverständnisses zu verstehen ist.

\subsection{Alterität als Instrument für die Bildungswissenschaften}

Um die einzelnen Elemente des hier erarbeiten alteritätsbezogenen Lernverständnisses zu bündeln und ihr analytisches Potential auf konkrete Lehr- und Lernkontexte übertragen zu können, wurde ein Modell entwickelt, das die gewonnenen Erkenntnisse zu explizieren und anzuwenden hilft. Zwei grundlegende Aspekte stehen in diesem Modell im Vordergrund: Zum einen, dass im Kern jeder Begegnung mit dem Anderen die wechselseitige Konstruktion des Andersseins des Ich mit einer weiteren Ebene des Andersseins steht. Zum anderen, dass in formalen Lernkontexten dem Anderssein der Anderen eine besondere Rolle zufällt. Diese Zusammenhänge möchte ich in Relation zum hier entwickelten und in Abbildung 4 grafisch gefassten Modell erläutern.

Grundlegendes Verständnis im Arbeiten mit dem Alteritätskonzept ist es, dass sich im Begegnen des Anderen die eigene Verortung als Subjekt widerspiegelt. Wenn ich etwas als anders erlebe, dann offenbart mir dies einen Anlass, um die eigenen Systeme des Wissens und seine handlungsleitenden Werte zu erkennen und damit (erneut) in einen Prozess der Aushandlung zu gehen. So ist jedem Begegnen des Anderen stets die Begegnung mit dem Anderssein des Ich inhärent. Sie steht im Mittelpunkt dessen, was die Begegnung mit dem Anderen - insbesondere in akademischen Lehr- und Lernkontext - zu bewirken vermag. So ist sie auch Kern des hier entwickelten Modells. In Auseinandersetzung mit ihr können die anderen Ebenen, über die ein Subjekt das Anderssein wahrnimmt, verhandelt werden: das Anderssein der Anderen, das Anderssein des Systems sowie die in Kapitel 2.2 vorgestellte und insbesondere für das Lernen als relevant eingestufte Ebene des Andersseins des Themas. Gemeinsam bilden diese vier Ebenen die Grundlage einer bildungswissenschaftlichen

Anwendung des Alteritätskonzeptes und explizieren, wie ein alteritätsbezogenes Lernverständnis operationalisiert werden kann. 


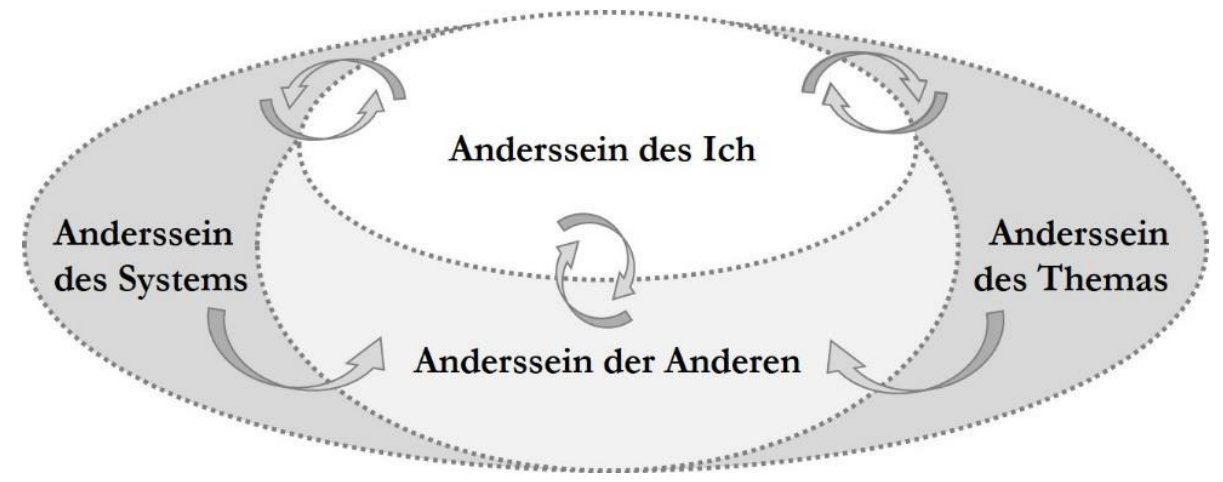

Abb. 4: Vier-Ebenen-Modell der Wahrnehmung und Wirkung im Begegnen des Anderen

Um die Möglichkeiten dieses Modells für den Bildungskontext Hochschule zu verdeutlichen, möchte ich hier die Wechselseitigkeit erläutern, die im Modell zwischen allen Ebenen des Andersseins und stets in Relation zum Anderssein des Ich zu beachten ist. Beginnen möchte ich mit der Verbindung zwischen dem Anderssein des Ich und dem Anderssein des Themas. Sie betrifft maßgeblich die im Lernen relevante Ebene der Kognition und des Erwerbs neuen Wissens. Als lernendes Individuum ist mir dieser Modus der Begegnung vertraut, kenne ich ihn doch im Grunde von allen Lernprozessen. Wahrgenommen wird das Anderssein eines Themas dann, wenn es dem vorhandenen Weltwissens eines Individuums nicht entspricht. Im Prozess des Abgleichs des eigenen Wissens mit dem noch unbekannten Neuen, erfolgt im Lernen schließlich der Schritt, in dem dieses Individuum das bestehende Wissenskonstrukt angleicht, erweitert oder - im Ablehnen der neuen Information - bestätigt. Aus Perspektive der Alterität kommt es im Zuge dieser Aushandlung zu Momenten, in denen das eigene Wissen hinterfragt und so ein Anderssein des Ich erfahren wird. Die Erkenntnisse, die in diesem Prozess erzeugt werden, geben dem lernenden Subjekt schließlich Gelegenheit, sich auf Basis eines bestätigten oder erweiterten Weltwissens gleichsam als Person (er)neu(t) zu verorten. Die Parameter der eigenen Identität und ihrer Rollen erfahren eine Überprüfung und so können auch die eigenen Perspektiven auf das In-der-Welt-Sein hinterfragt werden. Erneut wird deutlich, dass Lernen und Identität ineinander verwoben sind und sich - insbesondere in bildungsrelevanten Situationen - gegenseitig beeinflussen. Diese, in Kapitel 2.1 und 2.4 begründete Perspektive, wird vor dem Hintergrund des lernbezogenen Alteritätsmodells weiter verdeutlicht.

Im Modell findet sich die Wirkungskraft der Sozialisation in der interdependenten Beziehung des Andersseins des Ich mit dem Anderssein des Systems wieder. Da Sozialisation es ist, die den Handlungen einer Person in einem bestimmten Kontext Bedeutung gibt, ist es ihre Abwesenheit, die ein Nicht-Erkennen ebenjener Bedeutung bedingt. Indem ich das Verhalten in einem Kontext und so das dahinterliegende System als unbekannt und anders wahrnehme, wird für mich gleichsam das Anderssein des Ich erfahrbar. Während mir das System, das für seine Mitglieder sinnhaft und sinngebend wirkt, verborgen bleibt und mir somit verwehrt, ebenfalls bedeutungsvoll in ihm zu handeln, bietet dieser Zustand mir jedoch Gelegenheit, im Erfahren der Nicht-Verortung, die Qualitäten der eigenen Sozialisation zu erfassen und ihre Bedeutung 
für mein alltägliches Handeln zu reflektieren. Im Hochschulkontext bietet diese wechselseitige Beziehung zwischen Anderssein des Ich und des Systems insbesondere die Chance, Aspekte der eigenen akademischen Sozialisation zu ergründen.

Dem Anderssein der Anderen wird im hier entwickelten Modell eine Sonderrolle zugesprochen, da es in zweierlei Funktion mit dem Anderssein des Ich in Interaktion treten kann. Für sich allein ermöglicht diese Ebene der Wahrnehmung, ein anderes Subjekt in seinem Anderssein zu erfahren und so beispielsweise durch das Erkennen anderer Lernstrategien oder bevorzugter Lernmodi Rückschlüsse zum eigenen Lernverhalten zu ziehen. In Verbindung zu den Ebenen des Andersseins des Systems und des Themas kann der Begegnung mit dem Anderssein der Anderen zudem eine explizierende Funktion zukommen. Wodurch erkenne ich, dass ich ein System, z. B. die Bräuche in einem Vorlesungssaal in Deutschland, als anders wahrnehme? Dadurch, dass meine Kommiliton*innen klopfen und ich - nicht wissend, dass dies üblich ist - nicht. Wodurch erscheint mir ein Thema in einem Seminar als anders? Indem Andere es als für sie selbstverständliches Wissen diskutieren, wohingegen es mir unbekannt ist. Im Begegnen des Andersseins der Anderen wird somit transportiert und für das akademische Selbst sichtbar, dass man sich einem unvertrauten handlungsstrukturierenden System oder einem unbekannten Lerninhalt und somit einem Anderen gegenüber sieht. Nicht nur das, auch wird durch das Erleben des Andersseins des Systems oder Themas durch eine andere Person die Sinnhaftigkeit dieses Anderen legitimiert. Um erneut das deutschlandspezifische Beispiel des Klopfens in Vorlesungen zu bemühen: Durch das Verhalten der Menge erkenne ich, dass es offenbar eine Norm ist, am Ende der Sitzung in diesem Kontext zu klopfen. Eine Norm, die sich mir als Teil eines unbekannten Systems dadurch offenbart, dass sie von den Anderen selbstverständlich angewendet wird und mich dazu verleitet, in das Klopfen einzustimmen und so mein Verhalten zu ändern. Die soziale Eingebundenheit kann somit im Kontext eines unbekannten Systems dazu dienen, durch die Wirkmächte der Alterität ein relevantes Feedback zum eigenen Verhalten zu bekommen. Mit Blick auf die Gestaltung von Hochschullehre kann dies z. B. heißen, derlei Begegnungen mit dem Anderssein des Systems oder des Themas zu antizipieren, sie didaktisch mit kooperativen Lernsettings zu koppeln und so die von ihnen ausgelöste Unsicherheit bei den Studierenden abzumildern.

In der Begegnung des Andersseins der Anderen und den dort erfahrbaren Möglichkeiten, mit einem unbekannten Thema oder System umzugehen, können einem Individuum auf Ebene des Andersseins des Ich Wege aufgezeigt werden, sich dem Unbekannten zu nähern und zu eigen zu machen. Es ergeben sich beinahe nahtlos erste Hinweise darauf, wie ein solches Modell der Anwendung des Alteritätskonzeptes im akademischen Kontext Handlungsoptionen zu eröffnen vermag. Zunächst bedarf es jedoch, ein weiteres Element des Lernprozesses mit in diese Vorstellung aufzunehmen.

$\mathrm{Zu}$ Beginn dieses Rahmenpapiers habe ich ausgehend vom hier verwendeten Lernverständnis die Bedeutung der Reflexion für Prozesse des menschlichen Lernens skizziert. Sie ist es, überträgt man die Erkenntnisse des soeben eingeführten Modells weiter auf den Prozess des Lernens, die dem Lernen in der Begegnung mit dem Anderen seinen Wert gibt. In ihrer Qualität als nicht-intendierter Lernimpuls wird sie in der Begegnung mit dem 
Anderen erzeugt und ist dabei jenes Element, das im wahrgenommenen Anderssein des Ich zu wirken beginnt. Die Wirkmechanismen des oben vorgestellten Modells, das bewusst die Interaktion des Andersseins des Ich mit den anderen Anderssein-Ebenen ausdrückt, ergeben die hier implizierten und für ein nachhaltiges Lernen relevanten Reflexionsprozesse.

Alterität als Konstrukt, das die Verunsicherung des Selbst als wertvoll für seinen Fortbestand erklärt, erlaubt uns, die Relevanz der Reflexion in Lernprozessen auf einer weiteren subjektbezogenen Ebene zu explizieren und im Individuum selbst zu begründen.

Der Mehrwert dieser Erkenntnis ergibt sich insbesondere dann, wenn das hier zugrundeliegende Lernverständnis als Zugang gewählt wird, um Reflexionsimpulse verschiedener Qualitäten zu adressieren: als nicht-intendierter Impuls ebenso wie als bewusst strategische Handlung. Als analytischer Zugang kann ein alteritätsbezogenes Lernverständnis in Form des vorgestellten Modells zudem genutzt werden, um unerwartete Situationen im Rahmen einer nicht-intendierten Reflexion zu interpretieren. Als methodischer Ansatz fordert es weiterhin auf, die Begegnung mit dem Anderen und die hierbei ermöglichten Reflexionsimpulse zu antizipieren und didaktisch zu gestalten. Die Ebenen der Wahrnehmung des Andersseins sowie seiner Wirkung, wie sie im Modell gefasst werden, können hierfür als Ausgangspunkt dienen. Von ihnen abgeleitet ergibt sich eine Struktur zur Gestaltung alteritätsbezogener Reflexionsfragen, die nicht nur die einzelnen Ebenen des Andersseins zu adressieren vermag, sondern auch ihre jeweilige Wechselwirkung in den Blick nimmt.

Im Sinne eines reflexiven Dreischritts und ausgehend von der (antizipierten) Begegnung mit dem Anderen schlage ich vor, Reflexionsfragen mit drei Zielrichtungen zu gestalten. Zuvor ist jedoch folgendes zu bedenken: Zwar gehe ich davon aus, dass theoretisch alle Ebenen des Andersseins in akademischen Lehr- und Lernprozessen existieren und so reflektiert werden können, jedoch ist es durch ihre jeweilige Wechselwirkung ausreichend, den Zugang über die eine Ebene zu suchen, die zum Initiieren der Reflexion in einem Kontext besonders sinnvoll erscheint. Diese ergibt sich aus der jeweiligen Situation sowie dem Anderssein, das in ihr (erwartbar) hervorsticht. Auf Basis dieser initialen Ebene wird der Dreischritt durchlaufen.

1) Der erste Schritt der Reflexion ergründet den Inhalt des wahrgenommenen Andersseins. Hier kann die jeweilige Frage darauf ausgerichtet sein, was genau wahrgenommen wurde; was es war, dass eine Person hat innehalten lassen, was eine Routine gestört oder Verunsicherung ausgelöst hat.

2) In Schritt zwei richtet sich die Reflexionsfrage danach, den Ursprung der Wahrnehmung zu erkennen. Wodurch bin ich dem Anderssein begegnet? War es eine Person, die mich mit ihrem Verhalten ein Anderssein erkennen ließ oder war es ein anderer Faktor, dem ich in der Interaktion mit meiner Umwelt begegnete? 
3) Schließlich dient die dritte Reflexionsfrage dazu, der lernenden Person die Bedeutung des begegneten Anderen für sich selbst zu erkennen. Was sagt mir die Verwunderung über mein eigenes Handeln? Wessen kann ich mir dadurch bewusst(er) werden? Was möchte ich auf Basis dieses Innehaltens neu bedenken?

Im Durchlaufen des reflexiven Dreischritts kann die individuelle Wissensbasis der handelnden Person erweitert werden. ${ }^{66}$ Dies wiederum kann Auswirkung darauf haben, wie in zukünftigen Situationen dem Anderen begegnet und welches Anderssein dann - auf Basis des Gelernten wahrgenommen wird und werden kann. Der hier entwickelte, reflexive Dreischritt sowie die Parameter, die ihn rahmen, werden in Abbildung 5 veranschaulicht. Sein zyklischer Charakter wird durch die Abbildung hervorgehoben, ebenso die verschiedenen Ebenen des Andersseins, die er in ihrer möglichen Verbindung adressiert.

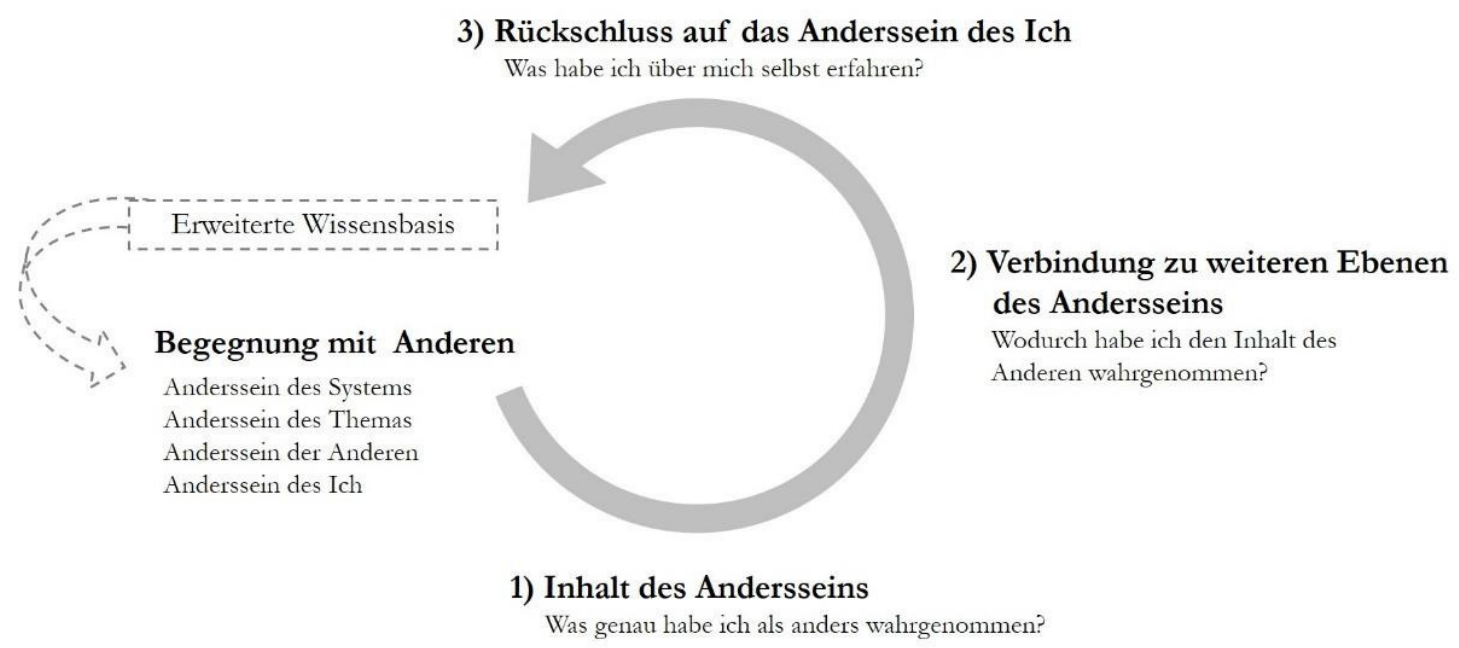

Abb. 5: Reflexiver Dreischritt zur Gestaltung alteritätsbezogener Reflexionsfragen

Für den Einsatz dieses Dreischritts ist aus meiner Sicht zu unterstreichen, dass Lernen in den formalen Settings der Hochschule in der Regel nicht isoliert, sondern in Gruppen stattfindet. So ist die Sonderrolle des Andersseins der Anderen bei der Gestaltung von Reflexionsfragen mitzudenken. Sie kann beispielsweise bereits in der Präsenzlehre sowie allgemein in diskursiven Formaten genutzt werden. Zudem ist aus Perspektive der Lehrperson zu beachten, dass ein Anderssein der Anderen nicht nur innerhalb der Gruppe der Studierenden, sondern auch durch Begegnung der Lehrperson erfolgen kann. Welche Auswirkungen dies aufgrund der asymmetrischen Machtbeziehung im Lehren (in Situationen der Bewertung) auf das damit

${ }^{66}$ In der Entwicklung des Dreischritts gehe ich einher mit Ashwin et al. (2015), die in ihrem Werk verdeutlichen, dass man sich im Lernen und Lehren der eigenen Identität bewusst sein und diese reflektieren sollte. 
verwobene Anderssein des Systems und des Themas haben kann, ist bei der Gestaltung der Reflexionsimpulse zu berücksichtigen. ${ }^{67}$

Gemeinsam ermöglichen das oben eingeführte Alteritätsmodell sowie der soeben vorgestellte reflexive Dreischritt die Anwendung eines alteritätsbezogenen Lernverständnisses auf den Hochschulkontext. Zusammenfassend lassen sich zwei Ebenen benennen, auf denen beide Instrumente genutzt werden können:

- rückblickend-analytisch: Erlebte Situationen in der Hochschullehre können aus einer Alteritätsperspektive beleuchtet und das Wahrgenommene lernbezogen ergründet werden. Während das Alteritätsmodell den Zugang darstellt, um Erlebtes bzw. Berichtetes zu analysieren, bietet der reflexive Dreischritt eine Struktur, um Prozesse der rückblickenden (Selbst)Reflexion anzuregen.

- antizipierend-gestaltend: Bereiche möglicher Begegnungen im Lernen \& Lehren sowie die ihnen inhärenten Ebenen des Andersseins können (in ihrer Verwobenheit) antizipiert und in der Planung beachtet werde. Erneut ermöglicht das Modell den Zugang zum Erkennen möglicher Ebenen des Andersseins, während der reflexive Dreischritt hier als Methode genutzt werden kann, um erwartete Situationen der Begegnung zu gestalten und lernförderlich zu beeinflussen.

Das Potential des alteritätsbezogenen Zugangs für die Bildungswissenschaften soll im folgenden Teil des Rahmenpapiers verdeutlicht werden. Die drei Studien, die im Rahmen dieser Forschung durchgeführt wurden, werden unter Verwendung der hier entstandenen Instrumente und mit Fokus auf beide Anwendungsebenen des alteritätsbezogenen Lernverständnisses kritisch beleuchtet. Welche Erkenntnisse bietet die Anwendung des Modells und die davon angeleiteten Reflexionsimpulse hinsichtlich der Alterität, die in den einzelnen Studien vorhanden war? Welcher lernförderliche Mehrwert wird durch eine solche Analyse sichtbar? Es ist dieser Teil des Rahmenpapiers, in dem das Erkenntnisinteresse meiner Forschung befriedigt wird.

Nach einer knappen (Re)Kontextualisierung der jeweiligen Studie, werden die in ihr zusammengetragenen Informationen mithilfe des Alteritätsmodells beleuchtet. Hierbei werden die drei oben verdeutlichten Ebenen der Anwendung berücksichtigt und sowohl analytisch-rückblickend als auch antizipierend-gestaltend argumentiert. So wird vorgestellt, welches Anderssein sich aufgrund des hier entwickelten Modells explizieren lässt und wie dieses Wissen im jeweiligen Kontext didaktisch genutzt wurde bzw. hätte genutzt werden können.

\footnotetext{
${ }^{67}$ Insbesondere, da Studien den Zusammenhang zwischen der sozialen Eingebundenheit von Lernenden mit der Lehrperson und dem situationalen Lerninteresse Ersterer belegen (vgl. hierzu Willems (2011, S. 298f.) und die Ausführungen in ihrer Studie zu Bedingungen des situationalen Interesses im Mathematikunterricht).
} 


\subsection{Die Anwendung}

\subsubsection{Teilstudie A: Verborgene Erwartungen \& akademische Sorialisation}

Die Sozialisation in einen disziplinären Kontext und die dort gelebten Werte und Normen ist Teil des Studierens. Dieses Einfinden in eine Fachkultur ist jedoch nicht überregional geltend, sondern stets durch lokale und - natürlich - individuelle Faktoren geprägt. Eine Erfahrung, die im Zuge der Internationalisierung der Hochschulen immer mehr Mitglieder einer Universität machen, sei es als Wissenschaftler*innen, die ihre Arbeitsstelle wechseln, oder als Studierende, die eine Zeit im Auslandsstudium absolvieren.

Auf Ebene des analytischen Zugangs, den das Alteritätsmodell anbietet, verdeutlichen die hier erhobenen Erfahrungen ehemalige Austauschstudierender, dass die wahrgenommenen Ausprägungen des bidden curriculum der Zieluniversität zu Begegnungen mit dem Anderen auf allen Ebenen führen konnte. Die Studierenden haben ein Anderssein des Ich, der Anderen und des Systems aufgrund der eigenen akademischen Sozialisation erfahren, die sie nicht immer wirkungsvoll handeln ließ und so im Agieren verunsicherte. Das Anderssein des Themas, das dem Studieren in gewissem Maße inhärent ist, hat für diese Gruppe eine weitere Erfahrung dargestellt. Grundlagen des Faches, die in der Lehre als selbstverständlich verhandelt wurden, unterschieden sich mitunter von denen, die an der Heimatuniversität als solche gelten und nun, im Auslandssemester, als Wissenslücke erfahren wurden. Insbesondere zwei Bereiche der erlebten Unsicherheit konnten im Zuge der Erhebung benannt werden: Erfahrungen mit den Gewohnheiten und Bräuchen in Lehr-Lernsettings und Erfahrungen mit Normen und Werten der Wissensproduktion. In der Interaktion mit Kommiliton*innen und Lehrenden wurde insbesondere das Anderssein der Anderen erlebt, wohingegen das Anderssein des Systems sich in den Aussagen zu strukturellen Aspekten wie der Vergabe von ECTS-Punkten oder der Organisation von Prüfungsleistungen offenbart. Durch Perspektive des Andersseins des Ich zeigt sich vor allem, welche Relevanz die Normen und Werte der Wissensproduktion des jeweiligen Kontextes haben. Dass diese, im Zuge der Sozialisation verinnerlichten Leitlinien akademischen Handelns, als Austauschstudent*in weder erwartet noch zunächst nachvollzogen werden können, wurde in Aussagen zu Art des Lehrens und Lernens deutlich. Sachverhalte und Informationen kritisch zu hinterfragen, Hilfestellungen beim Ergründen eigener Meinungen zu bekommen und den Fokus auf das eigene Verstehen zu legen, wurden als Erfahrungen beschrieben, in denen man sich selbst als anders erlebte und man, obgleich Teil der Gruppe, nicht konform zu agieren wusste.

Im Zuge dieses Forschungsprojektes wurde die antizipierend-gestaltende Ebene, die eine Anwendung des Alteritätsmodells ermöglicht, unmittelbar genutzt. War das übergeordnete Ziel doch nicht ausschließlich, das erlebte Andere aus studentischer Perspektive zu erfassen und zu systematisieren, sondern vielmehr basierend darauf, gezielte Reflexionsimpulse zum Ergründen impliziter Lehr-Erwartungen zu entwickeln und dadurch explizieren zu können, welche Werte das eigene Handeln leiten sowie welche Erwartungen sich daraus ergeben. ${ }^{68}$ So

68 Als Lehrperson die eigene Lehre so zu gestalten, dass sie allen im Raum vorhandenen akademischen Sozialisationen und individuellen Bedarfen gerecht wird, kann weder erreichbar noch Ziel sein. Vielmehr ist sinnvoll 
wurden mithilfe der ermittelten Bereiche der Begegnung mit dem Anderen Reflexionsfragen für Lehrende formuliert, die als Impulse zum Erkennen des Impliziten in der akademischen Lehre, in internationalen Kontexten sowie allgemein, genutzt werden können. Durch das Wissen um die Qualität des Konzepts der Alterität konnten durch Sammeln und Analysieren der Herausforderungen im Lernen jene Reflexionsimpulse ermittelt werden, die bei Studierenden und Lehrenden gleichermaßen ein Lernen über das eigene akademische Handeln und seiner Ursprünge ermöglichen: der eigenen akademischen Identität.

Übertragen auf die Gestaltung konkreter Lehr- und Lernsettings mit international zusammengesetzten Studierenden, bietet sich mit Blick auf das Modell weiter an, mit gezielten Reflexionsfragen in der Präsenz-, aber auch Selbstlernzeit den Fokus auf das Andere zu lenken und so einer möglichen Verunsicherung vorzubeugen. Statt einem Anderssein des Themas für sich alleine zu begegnen, könnte ein neues Thema mit einem Austausch darüber beginnen, welche (kanonisierten) Werke oder Theorien im eigenen Kontext verhandelt werden. Basierend darauf lässt sich nicht nur unterstreichen, dass die hier thematisierten Inhalte eine Auswahl sind, auch wird das Nicht-Gewählte bedacht und so Teil des Diskurses. Auch der Wert, den der Austausch über individuelle Lernwege und Perspektiven auf einen Lerninhalt bereithält, kann in derlei Kontexten gezielt gefördert werden. Hier können im Sinne des oben vorgestellten Dreischritts zur Gestaltung von Reflexionsimpulsen am Anderssein des Themas angeknüpft und bewusst die Verbindung zum Anderssein der Anderen gesucht werden (z. B. durch die Aufforderung, sich mit der Nachbarin bzw. dem Nachbarn darüber auszutauschen, in welchem Teil des gelesenen Textes man die Argumentation am wenigsten nachvollziehbar fand). Das Anderssein des Systems wiederum kann im gleichen Kontext in Verbindung zum wahrgenommenen Anderssein des Ich beachtet werden, indem man als Lehrperson beispielsweise um Einschätzung zu Etwas bittet und dabei gleichzeitig die eigenen Erwartungen kommuniziert (z. B. Die Diskussionen im Seminar sollen Sie darauf vorbereiten, auch in der Hausarbeit kritisch diskutieren zu können. Welche Darstellung in der vorliegenden Studie finden Sie am wenigsten überzengend?).

Aus den hier zusammengetragenen Erkenntnissen ergibt sich meines Erachtens ein weiteres Anwendungsfeld: die Unterstützung von Studierenden im Auslandssemester jenseits einer konkreten Lehrveranstaltung. So könnte für ankommende wie ins Auslandssemester aufbrechende Studierende ein Reflexionsbogen entwickelt und bereitgestellt werden, der ihnen ermöglicht, ausgehend vom wahrgenommenen Anderssein des Systems eigenständig und als lernbegleitende Strategie regelmäßig innezuhalten und so das Lernen im Kontext des Anderen auf Basis des reflexiven Dreischritts bewusst zu suchen, statt ihm nur zu begegnen.

Um diese ersten Gedanken zur Anwendung des alteritätsbezogenen Lernverständnisses in der eigenen Lehre weiter zu konkretisieren, hilft ein Blick in die Erkenntnisse der zweiten durchgeführten Studie dieses Forschungsprojektes, in der die Gestaltung des wahrgenommenen Anderen in der Lehre gleichsam Situation wie auch Forschungsfeld war.

und notwendig, die eigenen Erwartungen zu explizieren und das Einfinden in das lokale akademische System zu unterstützen (vgl. Carroll, 2015). 


\subsubsection{Teilstudie B: Akademisches Selbst \& kollektives Infragestellen}

Akademische Sozialisation ist auch in diesem Fallbeispiel der Ausgangspunkt. Durch sie wird die Identität eines Subjekts geprägt und mitunter um eine neue Ebene des Selbst erweitert. Hier, der des Akademischen. Dieses akademische Selbst leitet das Handeln jener Personen, die Hochschullehre gestalten: die Lehrenden und die Studierenden. Vor dem Hintergrund gesicherten Wissens um ihre jeweiligen Rollen und die damit verbundenen Verhaltensweisen begeben sie sich in Lehr- und Lernsettings und treten in Kontakt mit den dort verhandelten Themen. Hierbei stellt die kritische Auseinandersetzung mit neuen Inhalten, Theorien und Methoden für Studierende ein bekanntes Vorgehen im Lernen dar, das - wenngleich im individuellen Fall stets unterschiedlich herausfordernd - durch die Rahmung des bekannten Handlungsmodus und der Anleitung der Lehrenden bewältigt wird. Was aber, wenn dieser Rahmen entgegen vertrauter Normen und Werte, Handlungsroutinen und Erwartungen gestaltet ist? Wenn die erwartete Orientierung zur Einordnung und Bewertung des neuen Wissens durch die Lehrenden ausbleibt? Ein Umstand, der in der hier durchgeführten Studie über den Einsatz eines postkolonialen Ansatzes in der Lehre eintrat. Dieser Lehransatz zielt wie in Kapitel 3.2 vorgestellt darauf ab, vorhandene Zugänge der Wissenskonstruktion kritisch zu hinterfragen, darin enthaltene, normative Argumentationslinien zu ermitteln sowie einer epistemologischen Vielfalt Zutritt in die Lehrveranstaltung zu gewähren. Ein Vorgehen, das auf mehreren Ebenen die Begegnung mit dem Anderen evoziert und so nicht nur für Studierende, sondern auch für Lehrende eine Herausforderung dargestellt hat.

Die Anwendung des Alteritätsmodells in seiner Funktion als analytischer Zugang hat für diese Studie folgendes ergeben: Dem Anderssein des Ich ist in diesem Kontext, in dem sich alle Beteiligten in einer für sie ungewohnten, mitunter unberechenbaren Situation wiederfinden, zunächst keine besondere Wirkungskraft zugefallen. Diese offenbart sich jedoch in Relation zur Betrachtung des Andersseins der Anderen und insbesondere des Andersseins des Systems. Das gewohnte System des Lehrformats Seminar, die dort gelebten und erwarteten Rollen sowie die Teilhabe am Prozess des Lehrens und Lernens wurden in diesem Setting neu ausgehandelt. Der hierfür notwendige Reflexionsprozess über den Modus akademischen Studierens hat nicht nur den Zugang zu impliziten Ebenen der Interaktion geöffnet, sondern auch erlaubt in Frage zu stellen, was bislang als soziale Norm akzeptiert wurde. Das Wir der Gruppe konnte vor dem Hintergrund dieses anderen Systems neu begründet werden und so die Regeln gestalten, die im gemeinsamen Arbeiten von allen zu beachten waren. Ein Umstand, der die Verbindlichkeit des Miteinanders im gegebenen Beispiel verstärkt hat. Auf Basis der im Zuge des Andersseins des Systems neu ausgehandelten Parameter des sozialen Raumes Seminar wurde dem Anderssein der Anderen fortan als Reflexionsimpuls und Lernanlass begegnet. Unterschiedliche Zugänge zu einem Thema wurden diskutiert, vielfältige Deutungsmuster begründet und zueinander in Relation gesetzt, inhärente normative Gedanken gesucht und das wahrgenommene Anderssein des Themas verhandelt und dekonstruiert. Im bewusst erlebten Anderssein der Anderen sowie des Themas konnte sodann das Anderssein des Ich erfahren werden, ohne zu verunsichern. Vielmehr wurden dadurch Anlässe geschaffen, um sich in Abgrenzung 
zum Lernen der Anderen eigener Wissenskonstrukte und ihre Entstehung bewusst(er) zu werden. Der Zugang, um einen solch herausfordernden Lehransatz didaktisch sinnvoll zu gestalten, hat sich durch das Wissen um die soziale Einbettung des Lernens und der damit verbundenen Relevanz der Identität ergeben. So konnte das Augenmerk darauf gerichtet werden, dem akademischen Selbst der Studierenden eine Erweiterung des bekannten Rollenspektrums anzubieten und Gelegenheiten zu designen, um darin wirkungsvoll handeln zu können.

Gleichsam wie in der zuvor analysierten Teilstudie A, konnte die Anwendung des Modells bereits im Prozess der Durchführung antizipierend-gestaltend und insbesondere auf Ebene der Lehrenden erfolgen. Unter Verwendung des Wissens um die Chancen, die eine Begegnung mit dem Anderen innewohnen, wurde das geplante Lehrhandeln im Team hinterfragt und ebenso die Wissenskonzepte, die ein didaktisches Handeln motivierten. Das andere Team Teaching-Mitglied hat in diesem Kontext auf Ebene des Andersseins der Anderen wirken können, die, begründet im antizipierten Anderssein des Systems, das eigene Handeln als Lehrperson herausforderte und so ein Anderssein des Ich offenbaren konnte. Anlass hierfür waren die Reflexionsfragen, die im Anschluss an eine Sitzung gemeinsam im Team besprochen wurden:

„(1) What is the first thing you remember of today's class? (2) Which reaction by the group/a student surprised you the most? (3) At which time during class did you perceive the group as especially engaged; when as lost? (4) In which situation did you (as teaching team) struggle with the postcolonial approach; and why? (5) What will you keep in mind to support your students learning (needs) in the next session(s)?” (Thielsch, 2019a, S. 15, Fußnote 4).

In Relation zum vorgestellten Dreischritt zur Gestaltung von alteritätsbezogenen Reflexionsfragen ergibt sich der Wert dieser Fragen für die Mitglieder des Teaching Teams aus zwei Perspektiven: Im Austausch mit dem Team Teaching-Mitglied durchläuft eine Lehrperson die einzelnen Schritte initiiert durch das Anderssein der Anderen. Sie erfährt Inhalt dieses wahrgenommenen Andersseins, bringt dies in Verbindung mit Aspekten des Andersseins des Themas oder Systems und begegnet so dem Anderssein des Ich. In der Selbstreflexion alleine können die Leitfragen wiederum dazu dienen, sich selbst aus Perspektive des Andersseins des Ich entlang des Dreischritts zu reflektieren und zunächst Inhalt, dann Verwobenheit sowie Auswirkung des begegneten Andersseins zu ergründen.

Da parallel zur Durchführung dieser Studie die Entwicklung des Dreischritts in dieser Form noch nicht abgeschlossen war, wären die Leitfragen der Feldforschung aus heutiger Sicht zu erweitern. Das in ihnen implizit ausgelöste Erkennen des Andersseins des Ich, somit der dritte Schritt, wäre durch eine gezielte Frage zu integrieren und so in der Reflexion abzusichern.

\subsubsection{Teilstudie C: Gegenseitiges Begründen \& individuelles Erkennen}

Sowohl in der direkten Interaktion in einer Lehrveranstaltung als auch im gemeinsamen Vorund Nachbereiten... die Lehre als Teaching Team ist durchzogen von Impulsen, um die eigene 
Identität als Lehrperson sowie das individuelle Wissen über disziplinäre Praktiken oder Methoden der Lernförderung zu hinterfragen. Was in der zweiten Studie bereits anklingen konnte, wird durch die Analyse der dritten verdeutlicht. Hier haben die Erkenntnisse basierend auf den Daten einer quantitativen Erhebung zur Wahrnehmung Team Teaching-inhärenter Feedbackinformationen hervorgehoben, dass im gemeinsamen Lehren die Begegnung mit dem Anderen ein Bestandteil der Lehrrealität ist.

Wendet man das hier entwickelte Modell zur Analyse auf dieses Beispiel an, so ergibt sich zunächst aus Perspektive des Andersseins des Systems und des Themas, dass es herausfordernd sein kann, die Logiken und Werte im Lehrhandeln einer anderen Person in der eigenen Lehrveranstaltung realisiert zu sehen. Unerwartete Vorgehens- und Sichtweisen, aber auch inhaltliche Verweisstrukturen sowie bislang nicht (bewusst) verfolgte Ziele und wahrgenommene Erwartungen der Team Teaching-Partner*in können sichtbar werden und im Austausch zweierlei bewirken: Der wahrnehmenden Person offerieren sie den Impuls, die das eigene Handeln leitende Systematik zu hinterfragen, zu bestätigen oder anzugleichen. Der in ihrer Handlung wahrgenommenen Person wiederum bietet diese erfahrene Alterität einen Anlass, das Anderssein des Ich über die gespiegelte Fremdwahrnehmung des Team TeachingMitglieds nachzuvollziehen, das Bewusstsein für individuelle Ansätze und Überzeugungen zu schärfen und so in zukünftigem Handeln zu beachten. Ein Faktor, der sich in der Ausprägung dieses Wirkmechanismus im Rahmen der Studie als wertvoll herausgestellt, ist die Reaktion der Studierenden. Sie ist es, die das Anderssein des Ich ebenso wie das Anderssein der Anderen verdeutlicht, indem auf unterschiedliche Lehrstile unterschiedlich reagiert wird, das eine methodische Vorgehen unterstützt, einem anderen jedoch kritisch gegenüber gestanden wird. Während Prozesse, die das Selbst hinterfragen, aus Perspektive des Konzeptpaars der Alterität und Identität zunächst herausfordernd sein können, lässt sich im Kontext dieser Studie eine Chance nachzeichnen. Die Begegnung mit dem Anderen kann hier nicht nur direkt, sondern auch über das Dreiecksverhältnis der beiden Lehrenden in ihrer jeweiligen Lehrhandlung zu den Studierenden erfahren werden. Was dem einen Team Teaching-Mitglied im Vorgehen als Anderssein des Systems gespiegelt wird, erlebt auch das andere Mitglied und kann so durch die Rückmeldung der Studierenden an die andere Lehrperson gleichermaßen eine Feedbackinformation für sich gewinnen. So wirkt das Konzept der Alterität in diesem Beispiel (und wie es die Daten der durchgeführten Studie belegen) dadurch, dass es dreierlei Impulse zur Reflexion ermöglicht: Erstens durch direkte und indirekte Informationen der Team Teaching-Mitglieder untereinander, die aus Perspektive des Andersseins der Anderen Qualität und Ursprung des jeweils Anderen hinterfragen. Zweitens durch die Reaktion der Studierenden, die ein Anderssein des (Lebr)Systems oder des Themas im Handeln des Team Teaching-Mitglieds sichtbar machen. Und drittens durch die internen Feedbackprozesse, die dem Selbst im Erleben aller Perspektiven des Andersseins Anlass geben, sich als Lehrperson in der eigenen Rolle und dem jeweiligen Kontext neu zu verorten.

Auf einer antizipierend-gestaltenden Anwendungsebene bietet das Alteritätsmodell Anlass, sowohl Elemente in der hier verknüpften hochschuldidaktischen Qualifizierung als auch in der Begleitung der Teaching Teams zu ergänzen. So könnte das lernförderliche 
Potential durch die Begegnung mit dem Anderen beispielsweise unterstützt werden, wenn mittels des reflexiven Dreischritts ein Leitfaden gestaltet würde, der den Teams ermöglicht, die Impulse durch ein wahrgenommenes Anderssein im Rahmen ihrer Planungs- und Auswertungsgespräche zu nutzen. Das wahrgenommene Anderssein im Lehren könnte so entweder unmittelbar im Team besprochen oder vorbereitend auf den Austausch zunächst individuell ergründet werden. Gleichsam könnte in der Planung der einzelnen Workshops für die Gruppe der Lehreinsteiger*innen die Perspektive des alteritätsbezogenen Lernverständnisses eingesetzt werden, um bislang nicht genutzte Begegnungsebenen mit dem Anderen didaktisch einzubinden. Insbesondere im Austausch der Teilnehmenden untereinander kann beispielsweise ein wahrgenommenes Anderssein der Anderen dazu führen, das Anderssein verschiedener (Lebr)Systeme zu erkennen und die Sinnhaftigkeit von darin realisierten Lehrhandlungen zu thematisieren. Im Erkennen des Andersseins des Ich kann für die Teilnehmenden eine erweiterte Begründungslinie entstehen, um sich in ihrer Identität als Lehrende zu verorten.

\subsection{Zusammenführung und Diskussion}

Ein alteritätsbezogenes Lernverständnis auf das Handeln in akademischen Lehr- und Lernkontexten zu übertragen, ist durch das hier entwickelte Modell möglich. Die Auswertung der Teilstudien meiner Arbeit hat zudem sein Potential für unterschiedliche Einsatzfelder verdeutlicht. Als analytischer Zugang bietet es Lehrenden und Studierenden, Hochschuldidaktiker*innen und Bildungsforscher*innen Anlass, die in einem Kontext wahrgenommenen Ebenen des Andersseins zu ermitteln und ihren Inhalt sowie ihre Auswirkungen zu ergründen. Unterstützt durch die im hierfür entwickelten Dreischritt explizierte Struktur zur Gestaltung von Reflexionsfragen ist zudem ein Ausgangspunkt gegeben, um sich durch eine alteritätsbezogene Perspektive sowohl dem eigenen Lernen nähern zu können (als Instrument der Selbstreflexion) als auch dem Lernen einer anderen Person (als Instrument des Fragebogen- oder Interviewdesigns). So zeigt sich der Wert des Modells und des davon abgeleiteten Reflexionsdreischritts auf Ebene der Analyse ebenso wie auf Ebene der Gestaltung... und dies im Kontext einer lehrenden wie einer forschenden Tätigkeit.

Das Neue der hier vorgestellten Forschung ergibt sich folglich nicht nur in den Erkenntnissen der Einzelstudien, in denen die Begegnung mit dem Anderen nachgezeichnet und so die argumentative Grundlage zum Begründen eines alteritätsbezogenen Lernverständnisses gelegt werden konnte. Vielmehr verdeutlicht er sich in der Vielschichtigkeit, in der das Alteritätsmodell Anwendung finden kann und dadurch seine praktische Relevanz für unterschiedliche Zielgruppen ankündigt.

Mein Anliegen in dieser Arbeit war es, die Erkenntnisse der hier durchgeführten Forschung möglichst vielfältig mit der Praxis zu verschränken ${ }^{69}$ und ihre Anwendbarkeit und

${ }^{69}$ Die Relevanz, wissenschaftlichen Erkenntnisse der Hochschulforschung und hochschuldidaktischen Forschung zeitnah (und intensiver) für das Handeln als Hochschullehrende und Hochschuldidaktiker*innen zugänglich zu 
Relevanz dadurch hervorzuheben. Ein Ziel, das ich mit diesem Kapitel erreicht zu haben denke.

Kritisch zu bedenken geben möchte ich an dieser Stelle, dass - trotz seiner theoretischen Herleitung und der hier erfolgten Anwendung - der Prozess des Übertrags der Alterität als kulturwissenschaftlich-philosophisches Konzept sicher nicht abgeschlossen ist. Es wird weitere Übersetzungsprozesse brauchen, um das herausgearbeitete alteritätsbezogene Lernverständnis im bildungswissenschaftlichen Diskurs zu verankern. Insbesondere vor dem Hintergrund meiner eigenen initialen akademischen Sozialisation als Ethnologin, auf deren Grundlage die hier vollzogene Anwendung der Konzepte der Identität und Alterität auf den akademischen Lehr- und Lernkontext kein disziplinäres Umdenken, sondern lediglich ein Zusammendenken erforderte. Mögliche Leerstellen in der hier vorgestellten Encodierung des Alteritätskonzeptes in lerntheoretische Argumente sind daher nicht nur möglich, sondern vielmehr wahrscheinlich.

Im Sinne Halls (2006) ${ }^{70}$ bietet meine Arbeit eine Grundlage, um den aus meiner Perspektive vorhandenen Mehrwert der Anwendung des Alteritätskonzepts im Kontext akademischen Lehrens und Lernens erkennen zu können. Nun braucht es weitere Studien, um diesen Mehrwert nachzuzeichnen und Lernforscher*innen einen Anlass zu geben, das Wissen um Alterität auch in ihre Arbeiten zu übersetzen. Erste Hinweise dazu, wie und mit Blick worauf dies erfolgen kann, bietet das nächste Kapitel.

\section{Perspektiven - Was weiter zu bedenken wäre}

Das hier begründete Lernverständnis, das als Erweiterung subjektbezogener Perspektiven auf das Lernen um das Wissen zum Konzept der Alterität ergänzt wurde, offeriert im Bereich akademischer Lehr- und Lernkontexte verschiedene Ansatzpunkte zukünftiger Forschung. Skizzieren möchte ich an dieser Stelle drei von ihnen.

Zum einen kann die hier erarbeitet Grundlage um den Bereich affektiver Elemente im Lernen ergänzt und daran anschließend erneut kritisch reflektiert werden. Welche Emotionen sind es, die Individuen in der Begegnung eines Andersseins wahrnehmen? Inwiefern beeinflussen diese Emotionen, ob ein daraus generierter Impuls lernförderlich oder auch lernhinderlich genutzt wird? Welche affektive Reaktion löst ein wahrgenommenes Anderssein des Systems im Vergleich zum wahrgenommenen Anderssein der Anderen aus? Gründe, um eine solche Erweiterung hinsichtlich der affektiven Dimension vorzunehmen, ergeben sich aus verschiedenen Erkenntnissen der Lehr- und Lernforschung. So hebt beispielsweise Willems (2016, S. 238) in ihren Ausführungen zur Qualität des Lehrens und des professionellen

machen, hat Scholkmann (2017) vor einiger Zeit bereits nachdrücklich argumentiert. Zudem unterstreichen Lindblom-Ylänne \& Breslow (2017), dass empirische Belege ein wertvolles Instrument sind, um instinktives Handeln von Lehrenden zu bestärken (oder zu entkräften).

${ }^{70}$ Hall (2006) skizziert in seinem Beitrag „Encoding/Decoding“, dass man generell und so auch in der Wissenschaft, um kommunizieren zu können zunächst die jeweilige Information in das avisierte System hineincodieren muss. Erst dann könne sie erfolgreich durch Mitglieder dieses Systems wieder entziffert und gelesen, somit decodiert werden. 
Lehrhandelns hervor, dass eine Orientierung an den Lernbedürfnissen der Lernenden einen positiven Effekt auf Motivation und Wirksamkeit in Lernprozessen haben kann. Dass Emotionen das Lernbedürfnis einer Person beeinflussen können sowie gleichermaßen als Ausdruck derselben zu verstehen sind, verdeutlicht weiter, weshalb ein näheres Ergründen des Zusammenspiels der Begegnung mit dem Anderen und den damit zusammenhängenden Emotionen bedeutsam ist. Studien, die sich mit dieser Thematik beschäftigen, könnten daher um die Perspektive eine alteritätsbezogenen Lernverständnisses kritisch überprüft und ggf. ergänzt werden. ${ }^{71}$ Und dies nicht nur hinsichtlich wahrgenommener Gefühle im Lernen, sondern auch im Lehren. ${ }^{72}$

Daran anschließend ergibt sich weiter, das im Lernen handelnde Subjekt in Relation zu seiner Verortung als sozialisiertes Mitglied einer disziplinär und lokal ausgeprägten Wissenschaftsrealität näher zu betrachten. Eine solche Perspektive könnte Erkenntnisse über verschiedene Gruppen hervorbringen, so z. B. über Studierende, Lehrende und Hochschuldidaktiker*innen. Insbesondere der Blick auf die Verortung der Lehrenden und die Systeme, die ihr Handeln beeinflussen, erscheint mir als Fokus weiterer Forschungsprojekte sinnvoll. Sind es doch die Lehrenden, die das entscheidende Element in Veränderungsprozessen des Systems Hochschullehre sind. ${ }^{73}$ Zu verstehen, was sie in ihrem Handeln antreibt, überzeugt oder auch verunsichert, kann so nicht nur für die Entwicklung hochschuldidaktischer Angebote, sondern auch für die Umsetzung struktureller Maßnahmen an einer Institution wie der Hochschule bereichernd sein. Gleichsam könnte das hier entwickelte Modell einen vertieften Blick in bestehende Studien zur Entstehung von Lehridentitäten, individuellen Lehransätzen sowie existierender Modelle zur Lehrkompetenzentwicklung ermöglichen. ${ }^{74}$

Schließlich bietet die Anwendung des analytischen Modells der Alterität in Bildungskontexten einen Anknüpfungspunkt, um individuelle Lernprozesse in Relation subjektiver Vorwissensstrukturen und Wissenskonstruktionen bei der Aufnahme und Verarbeitung von Informationen zu ergründen. Fragen, die sich mir stellen, sind beispielsweise diese: Auf Basis welcher Lernimpulse entscheidet sich, welche Information wie verarbeitet wird, was davon Zutritt in das System eigener Wissenskonstrukte erhält und was aufgrund seines Andersseins als zu weit entfernt eingestuft wird und zugunsten der Parameter individueller Selbstverständnisse im Status des Anderen verharrt? Erste Einblicke, welche Hürden es zu bewältigen gilt, wenn Prozesse akademischen Lehrens und Lernens individuelle Selbstverständnisse derart herausfordern, dass die Konstruktion der eigenen Identität im Kern betroffen zu sein scheint, hat die Studien zum Einsatz eines postkolonialen Ansatzes bereits

\footnotetext{
${ }^{71}$ Vgl. u.a. Pekrun et al. (2011), Schmied \& Hänze (2016), Trigwell (2012) sowie Trigwell et al. (2012).

72 Beispielsweise in Relation zu Postareff \& Lindblom-Ylänne (2011) oder Kordts-Freudinger (2017).

73 Obgleich ihrer Bedeutung für Veränderungsprozesse auf Hochschulebene und insbesondere im Bereich der Curriculumentwicklung bekannt ist (vgl. u.a. Fleischmann et al., 2013), setzen strategische Maßnahmen nicht immer (auch) bei ihnen an. Ein Umstand, den Beelen (2017) hervorhebt, indem er die Lehrenden im Kontext der Prozesse der Internationalisierung der Curricula als „Missing Link“" bezeichnet.

${ }^{74}$ Vgl. u.a. Beijaard et al. (2004), Ho et al. (2001), Johannes \& Seidel (2012), Kember (1997), Prosser \& Trigwell (2006) sowie Trautwein (2013).
} 
zeigen können. Könnte eine derlei herausfordernde Begegnung mit dem Anderen besonderen Wert für das Lernen und Einfinden im Studium haben? Ist es nicht Ziel der Wissenschaft, kritische Auseinandersetzung zu fördern? Insbesondere mit Blick auf die Debatte um die Notwendigkeit (sowie mögliche Schwachstellen) der Kompetenzorientierung im Studium erscheint mir dies hoch relevant. Fordert die Ausrichtung auf Kompetenzen doch im Grunde (wenngleich unausgesprochen), dass wir unsere Studierenden auf Situationen vorbereiten, in denen sie auf bislang nicht erwartbare Weise die Teilbereiche ihrer Wissensstrukturen miteinander kombinieren müssen, um kompetent handeln zu können. ${ }^{75}$ Die Erkenntnisse, die ein alteritätsbezogenes Lernverständnis vor diesem Hintergrund ermöglichen kann, könnten so aufschlussreich für die Gestaltung einzelner Lehrformate, aber auch ganzer Studiengänge sein.

\section{Reflexion - Wo ich dem Anderen begegnet bin}

Es sind zwei Gedanken, die für die Erstellung dieses Kapitels richtungsweisend waren. Der erste ist, dass im Zuge einer intensiven Auseinandersetzung mit einem Thema, wie sie in Dissertationsprojekten angelegt ist, die Begegnung mit dem Anderen unweigerlich erfolgen kann (und auch soll). Der andere ist, dass es vermessen wäre, das eigene Lernverständnis von Erkenntnissen des Sozialkonstruktivismus abzuleiten, die soziale Einbettung des eigenen Forschungsprozesses aber nicht zu reflektieren. Beiden Gedanken möchte ich daher im Folgenden gerecht werden und mein Handeln sowie meine Erfahrungen im Rahmen dieser Arbeit kritisch reflektieren.

Als Wissenschaftlerin, die, basierend auf ihrer akademischen Sozialisation in die Sozialund Geisteswissenschaften und die methodologischen Ansätze der Ethnologie, seit einem Jahrzehnt im Feld der hochschuldidaktischen Bildungswissenschaften verortet ist, hat meine akademische Identität die vorliegende Arbeit in ihrer Entstehung maßgeblich geprägt. Der ethnologische Drang, die Sinnhaftigkeit und Struktur sozialer Systeme zu erfassen, ist hier nur ein Beispiel. In ihm begründet begegne ich der Vielschichtigkeit des Lehrens und Lernens im Hochschulkontext. Diese Vielschichtigkeit drückt sich für mich nicht nur in den Logiken ihrer disziplinären Systeme aus, sondern auch durch die Individuen, die in ihnen handeln. Um zu ergründen, welche Erkenntnisse die Verwendung eines Konzeptes wie der Alterität für das hochschuldidaktische Handeln bereithalten kann, war daher für mich leitend, in meiner Forschung gleichermaßen vielschichtig zu denken. Die unterschiedlichen Designs der hier vorgestellten Studien zeichnen sich durch eine entsprechende Breite aus und dies sowohl hinsichtlich der gewählten Forschungsfelder als auch der methodologischen Zugänge. Leitend für mich hierbei ist und war, die Sinnhaftigkeit verschiedener Forschungstraditionen zu bedenken und anzuerkennen.

\footnotetext{
75 Marylin Strathern (2008, S. 15) formuliert hierzu treffend, dass Hochschullehre letztlich avisiert „,[...] to prepare students for a real world where they have to be uncertain and still act, have doubts and still know what to do, encounter the unforeseen and carry on."
} 
Zwei für meine Arbeit richtungsgebende Überlegungen forschenden Handelns sollen an dieser Stelle expliziert werden: Im Sinne Mieke Bals (2012) skizzierter Forderung einer konzept-basierten Forschungspraxis, die sowohl der Interaktion mit dem jeweiligen Feld Beachtung schenkt als auch dem sich stets im Wandern wandelnden Charakter eines für die Forschung motivierten Konzeptes, ist mein methodisches Handeln fundiert in der analytischen Verwendung ebenjener Konzepte. So gehe ich davon aus, dass ein kritischer Blick auf disziplinär geprägte Konzepte in einem fachfremden Kontext ihre als intersubjektiv angedachten Eigenschaften zwar herausfordert, gleichsam aber bereichert und dadurch im Forschen methodologisch leitend sein kann.

Es ist darüber hinaus die gemahnte Offenheit der von John Laws (2004) formulierten Kritik an sozialwissenschaftlicher Forschung, die mein Tun prägt. Menschliches Handeln, so Law, erzeugt mitunter Phänomene, deren Komplexität nur schwer durch die Anwendung einer einzelnen Methode oder eines methodologischen Ansatzes durchdrungen werden kann. Wenn derlei Phänomene sich durch ihre Unberechenbarkeit und Nicht(ein)ordnung auszeichnen, wieso sollte die Erforschung derselben sich dann durch die Festlegung auf eine Methode selbst beschränken? Seinem herausfordernden Impuls - „Make a mess with methods“ (ebd.) nachzukommen, war und ist zwar nicht mein Anliegen, jedoch finde ich mich in der method(olog)ischen Offenheit, die sich in seinem Werk ausdrückt, als Forscherin wieder.

Ausgehend von beiden Überlegungen ist für mein forschendes Handeln als ethnologischsozialisierte Hochschuldidaktikerin charakteristisch, dass es auf eine Pluralität sozial- und geisteswissenschaftlicher Methoden zurückgreift, die in ihrer jeweiligen, mitunter überlappenden Anwendung in forschungsfeldrelevanten Konzepten begründet sind und theoretisch von ihnen geleitet werden. ${ }^{76}$ Basierend darauf sowie auf den jeweiligen Kontextspezifika der einzelnen Forschungsprojekte haben sich in allen drei Studien Hürden, aber auch Chancen ergeben. Als wohl größte Herausforderung ist hier zu nennen, sich je nach Studiendesign und Veröffentlichungsorgan einer anderen Sinnstruktur wissenschaftlicher Argumentation zu bedienen. An welcher Stelle erfolgt die theoretische Einordnung, wann und in welcher Form die Explikation des Erkenntnisinteresses, wie umfangreich die Darstellung des methodischen Vorgehens, zu welchem Grad die zwischen objektiver Darstellung, intersubjektiver Begründung und subjektiver Einordnung variierende Präsentation der Ergebnisse? Nicht nur, aber insbesondere im Zuge der Verschriftlichung (und dazugehörigen Review-Prozesse) ist so die Begegnung mit dem Anderen ein bemerkenswerter Bestandteil im Entstehen dieser Dissertation gewesen. Ein Bestandteil, der im Anfertigen dieser Arbeit einen eigenen Lernprozess dargestellt hat. Die Erkenntnisse dieses Lernens werde ich im Folgenden - natürlich - anhand des hier entwickelten Modells zur Anwendung des Alteritätskonzeptes auf Lernen im Hochschulkontext verdeutlichen.

76 Verweisen möchte ich an dieser Stelle auf die Beginn 2019 erschiene Zusammenstellung Schmohls (2019) über Kriterien und Bereiche hochschuldidaktischer Forschung und seinen Hinweis, dass diese insbesondere durch ihren Praxisbezug sowie ihre Feldnähe Bedeutung erhalten kann. 
In der Begegnung des Andersseins forschungslogischer Systeme hat mein Handeln als Wissenschaftlerin wertvolle Impulse erhalten, um das eigene Verständnis wissenschaftlichen Handelns zu hinterfragen und zu bereichern. Das zunächst wahrgenommene Anderssein des Ich im Annähern an die Logiken dieser Systeme ist im Prozess der Reflexion in neues Wissen über die epistemologischen Zugänge verschiedener Disziplinen gewichen und hat so meine Lesart unterschiedlicher Zugänge weiter geöffnet. Die Wertschätzung, die ich nun umso bewusster den verschiedenen Forschungslogiken und ihren Voraussetzungen entgegenbringen kann, hat meinen Blick auf die Sinnhaftigkeit dieser Zugänge sowie - und insbesondere - ihre Komplementarität bereichert. Eine Folge dieser Erkenntnis ist die im Prozess gewachsene Abfolge und Argumentationslinie der drei durchgeführten Studien. Nach der Annäherung und Beleuchten des Offensichtlichen, um mein Vorhaben weiter zu fundieren, haben sich Sinn und Wert der zweiten Studie (das nunmehr Antizipierte zu ergründen) fließend ergeben. Im dritten Design schließlich, dem Überprüfen des inzwischen begründbar Erwarteten, konnte sodann der Prozess der Erkenntnisgewinnung komplettiert werden. Ein Vorgehen, das mit einem einzigen methodologischen Ansatz so nicht hätte erfolgen können. Erst die Mischung diskursanalytisch-reflektierenden Handelns mit qualitativ-ethnographischen Methoden und schließlich der Genauigkeit quantitativer Operationalisierung hat die Argumentation dieser Arbeit (für mich) erzeugen können. Etwas, das mir ohne die Auseinandersetzung mit den jeweils anderen Forschungslogiken nicht bewusst und sicher nicht Teil zukünftiger Handlungen geworden wäre.

Das Anderssein des Themas hat für mich insbesondere in der Erstellung der letzten Studie und dem Prozess, die analytischen Möglichkeiten einer Statistiksoftware kennenzulernen, zu Wechselwirkung mit dem empfundenen Anderssein des Ich geführt. Unter uns: In dieser Phase ist der Gedanke, für einen quantitativen Ansatz einfach ,nicht gemacht zu sein“ mehrere Male hervorgerufen worden. Eine Wahrnehmung, die sich gewandelt hat. Das Zögern, dem Messen eines sozialen Phänomens zu vertrauen, ist im Zuge dieser Auseinandersetzung einer Befriedigung gewichen, durch ein entwickeltes Instrument Aspekte eines Phänomens in ihren Ausprägungen für eine Stichprobe in Relation zueinander setzen, gewichten und dies kommunizieren zu können.

Wie zu erwarten ist, konnte ich während der Entstehung dieser Arbeit sowohl das Anderssein des Systems als auch das des Themas über den Modus des Andersseins der Anderen erfahren. So waren insbesondere der Austausch und die Diskussionen im DoktorandinnenKolloquium, aber auch das Rückkoppeln und Teilhaben-Lassen von Kolleg*innen, Freund*innen und Familienmitgliedern an der jeweils erlebten Verwirrung bereichernd für mich. In der Rückschau würde ich sicher so manches Design bisheriger Forschungen auf Basis dieses neuen Wissens und meiner nunmehr (noch) bewussteren Verortung in der Rolle als Wissenschaftlerin anders ausrichten. Vor allem jedoch durch das nunmehr komprimierte Wissen, inwiefern das Konzept der Alterität meinen eigenen Lernprozess begleitet hat und nun durch das entstandene Instrumentarium weiter auf akademische Lehr- und Lernkontexte angewendet werden kann. 


\section{Mic Drop}

Eine Arbeit, die bewusst die soziale Konstruiertheit der Realität zu nutzen sucht, um die in ihr vorhandenen Phänomene des akademischen Lehrens und Lernens (noch) besser greifen zu können, scheint beim Anfertigen der dazugehörigen Ausformulierung nahezu widersprüchlich. Wie würde es möglich sein, im Nutzen der Sprache als Werkzeug der Verständigung ihre inhärenten Deutungsstrukturen zu umgehen? Bereits 1987 hat Bourdieu verdeutlicht (Bourdieu \& Wacquant, 2017, S. 274), dass die Sprache uns als Wissenschaftler*innen vor „dramatische Probleme“ stellt, da sie „,...] ein riesiger Vorrat von naturalisierten, [...] als solchen nicht erkannten Präkonstruktionen [ist], die als unbewusste Konstruktionswerkzeuge fungieren." Die sich mittels solcher Präkonstruktionen als Scheinevidenzen präsentierende Selbstverständlichkeiten bedürfen eines epistemologischen Bruchs (ebd., S. 284f.), um der Starrheit jener Begriffe und Konstrukte zu entkommen, die sonst aufgrund der eigenen akademischen Sozialisation im wissenschaftlichen Handeln nicht hinterfragt, folglich nicht bereichert würden.

Die Selbstverständlichkeit, mit der wir von der eigenen Identität, der Notwendigkeit individuellen Lernens sowie der Bedeutung des sozialen Kontextes im Lernen sprechen, sollte daher in meiner Arbeit aufgebrochen werden, um durch Verwendung des Konzeptes der Alterität einen neuen Pfad zum Ergründen lernrelevanter Aspekte aufzuzeigen.

So herausfordernd die Versprachlichung meiner Arbeit im Prozess des Schreibens war, so bereichernd war der interdisziplinäre und kontinuierlich nach konzeptuellen und methodologischen Brüchen suchende Prozess meines Handelns im Feld des Anderen. Im Rahmen meiner Arbeit hat der Begriff der Alterität Einreise in die akademische Gemeinschaft der Bildungsforschung, insbesondere der Hochschuldidaktik erhalten, und konnte als travelling concept im Sinne Mieke Bals eine konzeptuelle Erweiterung erfahren. Glücklicherweise! Denn Begriffe, so Bal (2006, S. 11), sind keine starren Gebilde. Durch ihren Gebrauch können sie vielmehr zwischen Anwendungsfeldern ebenso wie zwischen Disziplinen wandern. Und im Wandern „,...] müssen ihre Bedeutung, ihre Reichweite und ihr operationaler Wert nach jedem ,Ausflug'von neuem bewertet werden“ (ebd.).

Ich bin gespannt und freue mich darauf, welche weiteren Wanderbewegungen und Neubewertungen meine Arbeit zum Lernen und dem Anderen für das Konzept der Alterität auszulösen vermag. Mehr noch freue ich mich darauf, dem hierbei entstehenden Anderen erneut zu begegnen und davon zu lernen. 


\section{Bibliographie des Rahmenpapiers}

Agostini, E. (2016). Lernen im Spannungsfeld von Finden und Erfinden. Zur schöpferischen Genese von Sinn im Vollzug der Erfahrung. Paderborn, Ferdinand Schöningh.

Ahmed, S. (2000). Strange encounters. Embodied others in post-coloniality. London \& New York, Routledge.

Alheit, P. \& B. Dausien (2009). Bildungsprozesse über die Lebensspanne: Zur Politik und Theorie lebenslangen Lernens. In Handbuch Bildungsforschung, 713-734. Wiesbaden, VS Verlag für Sozialwissenschaften.

Argyris, C. \& Schön, D. (1974). Theory in practice: Increasing professional effectiveness. San Francisco, Jossey-Bass.

Ashwin, P. \& Associates (2015). Reflective teaching in higher education. London, New York, Oxford, New Delhi \& Sydney, Bloomsbury Academic.

Bal, M. (2006). Kulturanalyse. Frankfurt a.M., Suhrkamp Verlag.

Bal, M. (2012). Travelling concepts in the humanities. A rough guide. Toronto, Buffalo \& London, University of Toronto Press.

Barnett, R., \& R. Di Napoli (2008). Introduction. In Dies. (eds.), Changing identities in higher education: Voicing perspectives, pp. 1-8. London \& New York, Routledge.

Bedorf, T. (2011). Andere. Eine Einführung in die Sozialphilosophie. Bielefeld, transkript Verlag.

Benner, D. (1999). "Der Andere" und "Das Andere" als Problem und Aufgabe von Erziehung und Bildung. In Zeitschrift für Pädagogik, 45(3), 315-327.

Beijaard, D., Meijer, P. C., \& Verloop, N. (2004). Reconsidering research on teachers' professional identity. In Teaching and teacher education, 20(2), 107-128.

Beelen, J. (2017). The missing link in internationalisation: Developing the skills of lectures. In Zeitschrift für Hochschulentwicklung 12(4), 133-150.

Berger, P. L. \& T. Luckmann (1966). The social construction of reality. A treatise in the sociology of knowledge. New York, Anchor Books.

Bhabha, H. K. (2012 [1994]). The location of culture. London \& New York, Routledge.

Borst, E. (2011). Theorie der Bildung. Eine Einfübrung. Baltmannsweiler, Schneider Verlag Hohengehren.

Boud, D. \& Walker, D. (1990). Making the most of experience. In Studies in continuing education, 12(2), 61-80.

Boud, D. \& Walker, D. (1998). Promoting reflection in professional courses: The challenge of context. In Studies in higher education, 23(2), 191-206.

Bourdieu, P. \& L.J.D. Wacquant (2017). Reflexive Anthropologie. Frankfurt a.M., Suhrkamp.

Brandt, L., Reiffenrath, T. \& A. Thielsch (2019 i. E.). Begegnungen im Kontext Hochschullehre - Entdecken, reflektieren, nutzen. In Heuchemer, S., Szczyrba, B. \& T. van Treeck (eds.), Hochschuldidaktik und Hochschulentwicklung - Praxisperspektiven zwischen Profilbildung und Wertefragen. Bielefeld, wbv Verlag.

Bredella, L. (2010). Das Verstehen des Anderen. Kulturwissenschaftliche und literaturwissenschaftliche Studien. Tübingen, Gunter Narr Verlag. 
Brockbank, A. \& I. McGill (1998). Facilitating reflective learning in higher education. London, Society for Research into Higher Education.

Carroll, J. (2015). Tools for teaching in an educationally mobile world. London \& New York, Routledge.

Clifford, J., \& G. E. Marcus (eds.) (1986). Writing culture: The poetics and politics of ethnography. Berkley, Los Angeles \& London, University of California Press.

Cranton, P. (1996). Professional Development as Transformative Learning. New Perspectives for Teachers of Adults. San Francisco, Jossey-Bass.

Culhane, D. (2016). Imagining: An Introduction. In Elliott, D. \& D. Culhane (eds.), A different kind of ethnography. Imaginative practices and creative methodologies, S. 1-21. Toronto, University of Toronto Press.

Deimann, M. (2017). Relationierung von Bildung und Technik. Antrittsvorlesung im Rahmen des Habilitationsverfahrens. Hagen, deposit_hagen Publikationsserver der Universitätsbibliothek. Online verfügbar: https://ub-deposit.fernunihagen.de/servlets/MCRFileNodeServlet/mir_derivate_00001174/Deimann_Antrittsvorl esung_2017.pdf

Dewey, J. (1910). How we think. Boston, New York \& Chicago, D.C. Heath \& Co Publishers. Echterhoff, W. (2013). Erfahrung. Erfahrungsbildung. In M. A. Wirtz (Hrsg.), Dorsch. Lexikon der Psychologie (16. Auflage). Bern, Hogrefe Verlag.

Eickelpasch, R. \& C. Rademacher (2004). Identität. Bielefeld, transcript Verlag.

Entwistle, N. (2009). Teaching for Understanding at University. Deep Approaches and Distinct Ways of Thinking. London et al., Palgrave Macmillan.

Fairclough, N. (2003). Analysing Discourse: Textual analysis for social research. London, Routledge.

Faulstich, P. \& R. Bracker (2015). Lernen - Kontext und Biografie. Empirische Zugänge. Bielefeld, transcript Verlag.

Fink, L. D. (2013). Creating significant learning experiences: An integrated approach to designing college courses. San Francisco, Jossey-Bass.

Fleischmann, A. (2019). Grundprinzipien und Erfolgsfaktoren guter Hochschullehre. Keynote im Rahmen des Tages der Lehre der Leibnitz-Universität Hannover, 21.02.2019.

Fleischmann, A., Hein, C. \& A. Thielsch (2013). „Gehet hin und mehret euch!“ - Das Multiplikatorenprogramm der TU München zur Unterstützung engagierter Lehrender in den Fakultäten. In Schröttner, B. \& Ch. Hofer (eds.), Akademische Lehrkompetenzen im Diskurs. Theorie und Praxis - A Discourse on Academic Teaching Competencies, S. 126-140. Münster, Waxmann Verlag.

Futterer, K. (2012). Reflexion im Leistungsnachweis Lehrportfolio: Eine Herausforderung mit Potential. In Szczyrba, B. \& S. Gotzen (eds.), Das Lehrportfolio, S. 167-184. Münster, Lit Verlag.

Geertz, C. (2008). Thick description: Toward an interpretive theory of culture. In Oakes, T. \& P. L. Price (eds.), The Cultural Geography Reader, S. 41-51. London \& New York, Routledge. 
Göhlich, M. \& J. Zirfas (2007). Lernen. Ein pädagogischer Grundbegriff. Stuttgart, Kohlhammer Verlag.

Gruber, H. \& Rehrl, M. (2005). Praktikum statt Theorie? Eine Analyse relevanten Wissens zum Aufbau pädagogischer Handlungskompeten₹. Regensburg, Universität Regensburg.

Hall, S. (2006). Encoding/Decoding. In Durham, M. G. \& D. M. Kellner (eds.), Media and cultural studies. Key works (revised edition), pp. 163-173. Malden, Oxford \& Victoria, Blackwell Publishing.

Hattie, J. \& H. Timperley (2007). The power of feedback. In Review of educational research, 77(1), 81-112.

Hilzensauer, W. (2008). Theoretische Zugänge und Methoden zur Reflexion des Lernens. Ein Diskussionsbeitrag. In bildungsforschung, 5(2), 1-18.

Ho, A., Watkins, D., \& Kelly, M. (2001). The conceptual change approach to improving teaching and learning: An evaluation of a Hong Kong staff development programme. In Higher Education, 42(2), 143-169.

Huber, L. (1991). Sozialisation in der Hochschule. In Hurrelmann, K. \& D. Ulich (eds.), Neues Handbuch der Sozialisationsforschung, S. 417-441. Weinheim, Beltz Verlag.

Hurrelmann, K. \& U. Bauer (2015). Einführung in die Sozialisationstheorie. Das Modell der produktiven Realitätsverarbeitung. Weinheim \& Basel, Beltz Verlag.

Illeris, K. (2007). How we learn: Learning and non-learning in school and beyond. London \& New York, Routledge.

Illeris, K. (2014). Transformative learning and identity. London \& New York, Routledge.

Jarvis, P. (1992). Paradoxes of learning. On becoming an individual in society. San Francisco, JosseyBass.

Jenert, T. (2008). Ganzheitliche Reflexion auf dem Weg zu selbstorganisiertem Lernen. In bildungsforschung, 5(2), 1-18.

Jenert, T., Postareff, L., Brahm, T., \& Lindblom-Ylänne, S. (eds.). (2015). Enculturation and development of beginning students. In Zeitschrift für Hochschulentwicklung (ZFHE), 10(4).

Johannes, C., \& Seidel, T. (2012). Professionalisierung von Hochschullehrenden. In Zeitschrift für Erįiehungswissenschaft, 15(2), 233-251.

Kember, D. (1997). A reconceptualisation of the research into university academics' conceptions of teaching. In Learning and instruction, 7(3), 255-275.

Keller, R. (2011). Diskursforschung. Eine Einfübrung für SozialwissenschaftlerInnen. Wiesbaden, VS Verlag.

Kiehne, B. (2015). Die Biografie lehrt mit: eine qualitative Untersuchung zum Zusammenhang von Lernbiografie und Lebrüberzeugung bei Nachwuchslehrenden. Münster \& New York, Waxmann Verlag.

Killick, D. (2015). Developing the global student. Higher education in an era of globalisation. London \& New York, Routledge.

Klafki, W. (2007). Neue Studien zur Bildungstheorie und Didaktik: Zeitgemäße Allgemeinbildung und kritisch-konstruktive Didaktik. Weinheim, Beltz. 
Klinger, M., Thielsch, A. \& Wiemer, M. (2013). Kurzformate in der Hochschuldidaktik. Ein Ansatz zur Klassifizierung. In B. Berendt, A. Fleischmann, N. Schaper, B. Szczyrba \& J. Wildt (Hrsg.), Nenes Handbuch Hochschullehre (L 1.20). Berlin, DUZ Medienhaus.

Kolb, D.A. (1984). Experiential Learning. Englewood Cliffs, NJ, Prentice Hall.

Koller, H.-C. (2017). Grundbegriffe, Theorien und Methoden der Erriehungswissenschaft. Eine Einfübrung. Stuttgart, Kohlhammer Verlag.

Koller, H.-C. (2012). Bildung anders denken. Einfübrung in die Theorie transformatorischer Bildungsprozesse. Stuttgart, Kohlhammer Verlag.

Kordts-Freudinger, R. (2017). Feel, Think, Teach--Emotional Underpinnings of Approaches to Teaching in Higher Education. In International Journal of Higher Education, 6(1), 217-229.

Kuckartz, U. (2016). Qualitative Inbaltsanalyse. Methoden, Praxis, Computerunterstützung. Weinheim \& Basel, Beltz Verlag.

Künkler, T. (2011). Lernen in Beqiebung: Zum Verbältnis von Subjektivität und Relationalität in Lemprozessen. Bielefeld, transcript Verlag.

Law, J. (2004). After methods. Mess in social science research. London \& New York, Routledge.

Leask, B. (2015). Internationalizing the Curriculum. London \& New York, Routledge.

Light, G., Calkins, S. \& Cox, R. (2009). Learning and teaching in higher education: The reflective professional. Los Angeles et al., Sage.

Lindblom-Ylänne, S., \& Breslow, L. (2017). The importance of evidence-based enhancement of the quality of learning and teaching in research-intensive universities. In Strengthening Teaching and Learning in Research Universities, S. 187-213. Cham, Palgrave Macmillan.

Lindblom-Ylänne, S., Trigwell, K., Nevgi, A. \& Ashwin, P. (2006). How approaches to teaching are affected by discipline and teaching context. In Studies in Higher education, 31(03), 285-298.

Lusk, M. E., Sayman, D., Zolkoski, S., Carrero, K. \& C. L. Chui (2016). Playing well with others: Co-teaching in higher education. In The Journal of the Effective Schools Project, 23, $52-$ 61.

Mayring, P. (2014). Qualitative content analysis: theoretical foundation, basic procedures and software solution. Klagenfurt. Online verfügbar unter http:// nbn-resolving.de/urn:nbn:de:0168ssoar-395173

Mbembe, A. (2016). Decolonizing the university: New directions. In Arts and Humanities in Higher Education, 15(1), 29-45.

Meyer-Drawe, K. (2008). Diskurse des Lernens. München, Wilhelm Fink Verlag.

Meyer-Drawe, K. (2003). Lernen als Erfahrung. Zeitschrift für Eræiehungswissenschaft, 6(4), 505514.

Mezirow, J. (1991). Transformative dimensions of adult learning. San Francisco, Jossey-Bass.

Mezirow, J. (2000). Learning to think like an adult. Core concepts of transformation theory. In Mezirow, J. \& Associates (eds.), Learning as transformation, S. 3-34. San Francisco, Jossey-Bass.

Müller-Funk, W. (2016). Theorien des Fremden. Tübingen, A. Francke Verlag. 
Olsson, T., Mårtensson, K., Roxå, T. \& Ahlberg, A. (2012). Pedagogical competence and teaching skills - a development perspective from Lund University, Sweden. In Szczyrba, B. \& S. Gotzen (eds.), Das Lehrportfolio, S. 2459-259. Münster, Lit Verlag.

Pekrun, R., Goetz, T., Frenzel, A. C., Barchfeld, P., \& Perry, R. P. (2011). Measuring emotions in students' learning and performance: The Achievement Emotions Questionnaire (AEQ). In Contemporary educational psychology, 36(1), 36-48.

Postareff, L., \& Lindblom-Ylänne, S. (2011). Emotions and confidence within teaching in higher education. In Studies in Higher Education, 36(7), 799-813.

Prosser, M., \& Trigwell, K. (2006). Confirmatory factor analysis of the approaches to teaching inventory. In British journal of educational psychology, 76(2), 405-419.

Ramsden, P. (1997). The context of learning in academic departments. The experience of learning, 2, 98-216.

Rhein, R. (2015). Hochschulisches Lernen - eine analytische Perspektive. In Zeitschrift für Weiterbildungsforschung, 38(3), 347-363. DOI 10.1007/s40955-015-0042-9

Rorty (2017 [1987]). Der Spiegel der Natur. Eine Kritike der Philosophie. Suhrkamp.

Rychen, D. S. E. \& Salganik, L. H. E. (2001). Introduction. In Dies. (eds.), Defining and selecting key competencies, pp. 1-15. Seattle, Toronto, Bern \& Göttingen, Hogrefe \& Huber Publishers.

Sartre, J.-P. (2009). Das Sein und das Nichts. Versuch einer phänomenologischen Ontologie [in neuer Übersetzung]. Hamburg, Rowohlt Taschenbuch Verlag.

Said, E. (2003 [1978]). Orientalism. London, Penguin.

Schäfer, A. (2004). Alterität: Überlegungen zu Grenzen des pädagogischen Selbstverständnisses. Zeitschrift für Pädagogik, 50(5), 706-726.

Schloos, U. (2000). Der Kreislauf des Erfolgs: Materialien zum Selbstmanagement für Lernende. Bonn, Deutsches Institut für Erwachsenenbildung. Online verfügbar: https://www.diebonn.de/esprid/dokumente/doc-2000/schloos00_01.pdf

Schmidt, J. (2016). Identitätskonstruktion und Verfasserreferen₹ in deutschen und US-amerikanischen wissenschaftlichen Artikeln. Göttingen, eDiss. http://hdl.handle.net/11858/00-1735-00000028-8813-2

Schmied, V., \& Hänze, M. (2016). Testtheoretische Überprüfung eines Fragebogens zu Kompetenzen der Selbst- und Studienorganisation und lernrelevanten Emotionen bei Studierenden. In die hochschullehre, 2, 1-16.

Schmohl, T. (2019). Hochschuldidaktische Begleitforschung. Perspektiven auf die wissenschaftliche Analyse dynamischer Lehr- und Lernsettings. In Schmohl, T., Schäffer, D., Kieu-Anh, T. \& B. Eller-Studzinsky (eds.), Selbstorganisiertes Lernen an Hochschulen Strategien, Formate und Methoden, S. 179-189. Bielefeld, wbv Verlag. DOI: 10.3278/6004678w

Scholkmann, A. (2017). Bridging the gap: Research as a practice to link higher education research and educational development. In die hochschullehre, 3, 1-15.

Schön, D. A. (1983). The Reflective Practitioner. How professionals think in action. New York, Basic Books. 
Schön, D. A. (1987). Educating the Reflective Practitioner. Toward a New Design for Teaching and Learning. San Francisco, Jossey-Bass.

Spivak, G. C. (1994). Can the subaltern speak? In: Williams P. \& L. Chrisman (eds.), Colonial Discourse and Post-Colonial Theory: A Reader, pp. 66-111. New York, Colombia University Press.

Strathern, M. (2008). Preamble: knowledge identities. In Barnett, R. \& R. Di Napoli (eds.), Changing identities in higher education. Voicing Perspectives, pp. 11-25. New York, Routledge.

Takkaç Tulgar, A. (2019). Exploring the bi-directional effects of language learning experience and learners' identity (re) construction in glocal higher education context. Journal of Multilingual and Multicultural Development, 1-16. DOI: 10.1080/01434632.2019.1611837

Taylor, C. A. (2017). Is a posthumanist Bildung possible? Reclaiming the promise of Bildung for contemporary higher education. In Higher Education, 74 (3), 419-435.

Taylor, P. (2008). Being an academic today. In Barnett, R. \& R. Di Napoli (eds.), Changing identities in higher education. Voicing Perspectives, pp. 27-39. New York, Routledge.

Tennant, M. (2012). The learning self: Understanding the potential for transformation. San Francisco, Jossey-Bass.

Thielsch, A. (2016). Lehrerfahrung teilen, Lehrkompetenzen erweitern - Team Teaching als Angebot zur Lehrkompetenzentwicklung bei Promovierenden und Professor/innen. In Hartz, S. \& S. Marx (eds.), Leitkonzepte der Hochschuldidaktik: Theorie - Empirie - Praxis, S. 15-24. Bielefeld, W. Bertelsmann Verlag.

Thielsch, A. (2017). Approaching the Invisible. Hidden Curriculum and Implicit Expectations in Higher Education. In Zeitschrift für Hochschulentwicklung 12(4), 167-187. DOI:10.3217/zfhe-12-04/11.

Thielsch, A. (2019a). Listening Out and Dealing with Otherness. A Postcolonial Approach to Higher Education Teaching. In Arts and Humanities in Higher Education. February 26, 2019; DOI: $10.1177 / 1474022219832459$.

Thielsch, A. (2019b). Feedback in Team Teaching-Konstellationen. Eine Studie zur Lehrkompetenzentwicklung durch Begegnung anderer Wissensformen. In die hochschullehre, 5, 161-186.

Thielsch, A. (2019c i. E.). Alterität. In Wirtz, M. A. (ed.), Dorsch. Lexikon der Psychologie (19. Auflage). Bern, Hogrefe Verlag.

Trautwein, C. (2013). Struktur und Entwicklung akademischer Lehrkompetenz. In Heiner, M. \& J. Wildt (eds.), Professionalisierung der Lebre. Perspektiven formeller und in-formeller Entwicklung von Lebrkompetenzim Kontext der Hochschulbildung, S. 83-129. Bielefeld, W. Bertelsmann Verlag.

Trautwein, C. \& M. Merkt (2013). Struktur und Entwicklung von Lehrkompetenz im Spannungsfeld von Überzeugungen, Konzepten und Praxis von Lehren und Lernen. In Heiner, M. \& J. Wildt (eds.), Professionalisierung der Lehre. Perspektiven formeller und in-formeller Entwicklung von Lebrkompetenz im Kontext der Hochschulbildung, S. 179-210. Bielefeld, W. Bertelsmann Verlag. 
Tremp, P. (2012). Universitäre Didaktik: Einige Überlegungen zu Lehrkompetenzen an Universitäten. In Egger, R. \& M. Merkt (eds.), Lernwelt Universität (pp. 15-28). Wiesbaden, Verlag für Sozialwissenschaften.

Trigwell, K. (2012). Relations between teachers' emotions in teaching and their approaches to teaching in higher education. Instructional Science, 40(3), 607-621.

Trigwell, K., Ellis, R. A., \& Han, F. (2012). Relations between students' approaches to learning, experienced emotions and outcomes of learning. Studies in Higher Education, $37(7), 811-824$.

Waldenfels, B. (2013 [1990]). Der Stachel des Fremden. Frankfurt a. M., Suhrkamp.

Watzlawick, P., \& Beavin, J. (1967). Some formal aspects of communication. American Behavioral Scientist, 10(8), 4-8.

Weimer, M. (2002). Learner-centered teaching: Five key changes to practice. San Francisco, JosseyBass.

Weinert, F. E. (2001). Concept of competence: A conceptual clarification. In Rychen, D. S. \& L. H. Salganik (eds.), Defining and selecting key competencies, pp. 45-65. Seattle, Toronto, Bern \& Göttingen, Hogrefe \& Huber Publishers.

Wenger, E. (1998). Communities of practice: Learning, meaning, and identity. Cambridge university press.

Wiemer, M. (2012). Reflection anticipating action: Das Lehrportfolio zum Einstieg in die Lehre. In Szczyrba, B. \& S. Gotzen (eds.), Das Lehrportfolio, S. 209-216. Münster, Lit Verlag.

Willems, A. S. (2011). Bedingungen des situationalen Interesses im Mathematikunterricht. Eine mehrebenenanalytische Perspektive. Münster, Waxmann Verlag.

Willems, A. S. (2016). Unterrichtsqualität und professionelles Lehrerhandeln. Prozesse und Wirkungen guten Unterrichts aus dem Blickwinkel der empirischen Schul- und Unterrichtsforschung. In Porsch, R. (eds.), Einführung in die Allgemeine Didaktik: Ein Lehrund Arbeitsbuch für Lebramtsstudierende, S. 237-289. Münster \& New York, Waxmann Verlag.

Van Manen, M. (1991). The Tact of Teaching. The Meaning of Pedagogical Thoughtfulness. New York, Routledge.

Young, R. J. (2003). Postcolonialism: A very short introduction. Oxford, Oxford University Press. 


\section{Kurzvorstellung der Teilstudien}

\section{Teilstudie A: Das Offensichtliche beleuchten}

Approaching the Invisible:

Hidden Curriculum and Implicit Expectations in Higher Education

\begin{tabular}{|c|c|c|}
\hline \multicolumn{2}{|c|}{ Aspekt } & Beschreibung \\
\hline \multirow{5}{*}{ 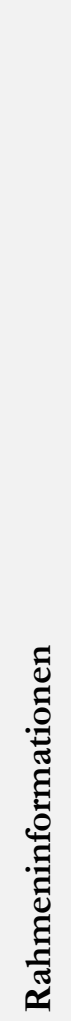 } & $\begin{array}{l}\text { Vollständige } \\
\text { Referenz }\end{array}$ & $\begin{array}{l}\text { Thielsch, A. (2017). Approaching the Invisible: Hidden Curriculum } \\
\text { and Implicit Expectations in Higher Education. In Zeitschrift für } \\
\text { Hochschulentwicklung, 12(4), 167-187. DOI:10.3217/zfhe-12-04/11. }\end{array}$ \\
\hline & Schlagworte & $\begin{array}{l}\text { Hidden curriculum in higher education, individual learning (and) } \\
\text { experience, exchange students, critical discourse analysis }\end{array}$ \\
\hline & $\begin{array}{l}\text { Publikationsorgan } \\
\text { und Auswahl }\end{array}$ & $\begin{array}{l}\text { Die Zeitschrift für Hochschulentwicklung (ZFHE) ist ein international } \\
\text { aufgestelltes Open Access Journal, das regelmäßig und unter } \\
\text { wissenschaftlicher Schirmherrschaft Herausgaben zu aktuellen } \\
\text { Themen im Bereich Hochschule organisiert; die Begutachtung } \\
\text { erfolgt über ein double blind Peer Review. } \\
\text { Die Wahl fiel auf dieses Journal, da es im hochschuldidaktischen } \\
\text { Kontext weit rezipiert wird und Ende } 2016 \text { einen zur Forschung } \\
\text { passenden Call veröffentlichte. }\end{array}$ \\
\hline & Publikationsstatus & Erschienen. \\
\hline & Publikationshistorie & $\begin{array}{l}\text { Forschungsdesign Beginn 2017, Erhebung Frühjahr 2017, } \\
\text { Einreichung Juni 2017, erschienen Dezember } 2017 .\end{array}$ \\
\hline 离 & $\begin{array}{l}\text { Abstract } \\
\text { (wie veröffentlicht) }\end{array}$ & $\begin{array}{l}\text { In situations of colliding discursive practices, their respective } \\
\text { qualities can emerge. Based on this assumption, a research project } \\
\text { has been conducted at the University of Göttingen that aimed at } \\
\text { better understanding the hidden curriculum experienced by former } \\
\text { exchange students. Using critical discourse analysis and an inductive } \\
\text { approach as foundation, statements of this group have been } \\
\text { collected and analysed. Implications regarding the experienced } \\
\text { hidden curriculum were used to develop a reflective guideline for } \\
\text { academic teaching staff. This paper introduces the theoretical and } \\
\text { methodological foundation of my research and offers insight in the }\end{array}$ \\
\hline
\end{tabular}




\begin{tabular}{|c|c|}
\hline & $\begin{array}{l}\text { findings on how to approach challenges caused by the implicit nature } \\
\text { of the hidden curriculum. }\end{array}$ \\
\hline Erkenntnisinteresse & $\begin{array}{l}\text { Wodurch kann das wahrgenommene sowie implizit gelebte bidden } \\
\text { curriculum ermittelt und benannt werden? } \\
\text { Wie können Hochschullehrende dabei unterstützt werden, basierend } \\
\text { auf den Erkenntnissen über dieses bidden curriculum die eigenen } \\
\text { Erwartungen und die ein solches Curriculum (re)produzierenden } \\
\text { Handlungen zu erkennen? }\end{array}$ \\
\hline Forschungsfeld & $\begin{array}{l}\text { Internationalisierung der Hochschullehre, retrospektive Erfahrungen } \\
\text { Austauschstudierender sowie Lehrender der Universität Göttingen }\end{array}$ \\
\hline $\begin{array}{l}\text { Aufbau und } \\
\text { Argumentation }\end{array}$ & $\begin{array}{l}\text { Im einleitenden Teil (Introduction) wird die Arbeit im aktuellen } \\
\text { Forschungsfeld der Internationalisierung der Curricula und ihrem } \\
\text { vielschichtigen Verständnis universitärer Lehrpläne (formale, } \\
\text { informelle, verborgene bzw. hidden Curricula) verortet und die } \\
\text { Herleitung dafür geboten, dass das implizite bidden curriculum in } \\
\text { Situationen widersprüchlicher Erwartungen sichtbar wird. Das } \\
\text { Erkenntnisinteresse und Vorgehen wird hier skizziert, bevor es im } \\
\text { zweiten Teil (On Academic Learning (and) Discourses) des } \\
\text { Papers theoretisch fundiert wird. Ausgehend von der sozialen } \\
\text { Einbettung individuellen Lernens wird argumentiert, inwiefern im } \\
\text { Lernen die Sozialisation in einen Lernkontext relevant ist. Durch sie } \\
\text { wird das wirkungsvolle Handeln innerhalb eines Diskurses } \\
\text { begründet, so die Annahme, und gleichsam die Teilhabe sowie } \\
\text { (Re)Produktion eines lokalen akademischen Lehr-Lerndiskurses und } \\
\text { der darin verborgenen Normen und Werten ermöglicht. Ausgehend } \\
\text { von den Erkenntnissen zum individuellen Lernen wird dargestellt, } \\
\text { dass die hier genutzten Instrumente - die Erhebung bei ehemaligen } \\
\text { Austauschstudierenden ebenso wie die Entwicklung eines } \\
\text { Reflexionsbogens für Lehrende - Bezug auf drei veranschlagte } \\
\text { Elemente bedeutungsvoller Lernprozesse nehmen: dem } \\
\text { Vorhandensein einer abstrakten Information, einer konkreten } \\
\text { Erfahrung und einer beides in Relation setzenden Reflexion. } \\
\text { Schlussendlich erfolgt die wohl wichtigste Einordnung der Arbeit als } \\
\text { Teil kritischer Diskursanalyse. Diese zielt als Teil des größeren } \\
\text { Feldes diskursanalytischer Wissenschaft darauf ab, die dialektische } \\
\text { Verbindung sozialer Praktiken und Diskursen zu ermitteln, möchte } \\
\text { jedoch zudem inhärente (Macht)Strukturen sichtbar machen und so } \\
\text { ein Aufbrechen derselben bewirken. } \\
\text { Im folgenden Teil (Tracking Down the Hidden Curriculum) wird } \\
\text { in drei Schritten die Erhebung und Analyse der Erfahrungen von }\end{array}$ \\
\hline
\end{tabular}




\begin{tabular}{|c|c|}
\hline & $\begin{array}{l}\text { Austauschstudierenden nachgezeichnet und ihre Ergebnisse } \\
\text { vorgestellt. Der zuvor antizipierte Einfluss der akademischen } \\
\text { Sozialisation für die Begegnung eines lokalen bidden curriculum wurde } \\
\text { anhand der Daten spezifischen Bereichen des Hochschulstudiums } \\
\text { zugeordnet und drauf basierend in Teil vier der Arbeit (Making } \\
\text { Expectations Explicit) als Ausgangspunkt für die Entwicklung } \\
\text { eines Reflexionsbogens für Lehrende genutzt. Die antizipierte } \\
\text { Zielrichtung von neun, zu den Ergebnissen der vorherigen } \\
\text { Erhebung komplementären Reflexionsfragen wird vorgestellt und } \\
\text { die Annahme ihres Funktionierens über die Analyse der Antworten } \\
\text { einer Gruppe Lehrender, die den Bogen testeten, begründet. } \\
\text { Im abschließenden Fazit (Conclusion) wird neben einer kurzen } \\
\text { Zusammenfassung der Erkenntnisse unterstrichen, weshalb die } \\
\text { Individualität in Lern- und Lehrbiographien eine Vielzahl von } \\
\text { Ausprägungen verborgener Lehrpläne entstehen bzw. wahrnehmbar } \\
\text { machen kann und dass ein sich Annähern über die gezielte Reflexion } \\
\text { von Erfahrungen der Begegnung anderer Erwartungen in } \\
\text { akademischen Kontexten hilft, sich der eigenen bewusst(er) zu } \\
\text { werden. }\end{array}$ \\
\hline $\begin{array}{l}\text { Methodisches } \\
\text { Vorgehen }\end{array}$ & $\begin{array}{l}\text { Verortet als Forschung im Sinne der kritischen Diskursanalyse; } \\
\text { Datenerhebung und -auswertung der Erfahrungen ehemaliger } \\
\text { Austauschstudierender mithilfe eines qualitativ ausgerichteten } \\
\text { Online-Fragebogens: } \\
\text { - acht personenbezogene Items (insb. zur individuellen } \\
\quad \text { Lernbiographie), } \\
\text { - drei offene Fragen zu Erfahrungen im Auslandssemester } \\
\quad \text { unter Verwendung dreier Reflexionsimpulse [Vergleich, } \\
\quad \text { Herausforderung, Rat]); } \\
\text { Inhaltsanalytische Auswertung der qualitativen Daten mittels } \\
\text { induktiver Kategorienbildung; } \\
\text { Inhaltsanalytische Auswertung der Lehrenden-Angaben im } \\
\text { getesteten Reflexionsbogen mittels deduktiver Kategorienbildung } \\
\text { (basierend auf den Kategorien, die zuvor im Datenmaterial } \\
\text { studentischer Aussagen ermittelt wurden). }\end{array}$ \\
\hline $\begin{array}{l}\text { Zentrale } \\
\text { Erkenntnisse }\end{array}$ & $\begin{array}{l}\text { Anhand der Reflexionsimpulse des Vergleichs, der Herausforderung } \\
\text { und des Rates an eine andere Person konnten Erfahrungen } \\
\text { ehemaliger Austauschstudierender erhoben werden, die } \\
\text { Rückschlüsse auf den qualitativen Gehalt des wahrgenommenen } \\
\text { bidden curriculum an einer Universität ermöglichen. Diese spiegeln den } \\
\text { Einfluss der akademischen Sozialisation auf Erwartungen an Lehr- }\end{array}$ \\
\hline
\end{tabular}




\begin{tabular}{|c|c|c|}
\hline & & $\begin{array}{l}\text { Lernsituationen wider und geben Zeugnis ab, welche } \\
\text { Verunsicherung die Begegnung anderer, widersprüchlicher } \\
\text { Erwartungen auslösen. Die gezielte Reflexion eigener akademischer } \\
\text { Werte und Normen einerseits und erfahrener Begegnungen } \\
\text { kollidierter Erwartungen in internationalen Lehrkontexten } \\
\text { andererseits, kann Lehrenden ermöglichen, den Herausforderungen } \\
\text { durch das gelebte bidden curriculum entgegenzuwirken. }\end{array}$ \\
\hline 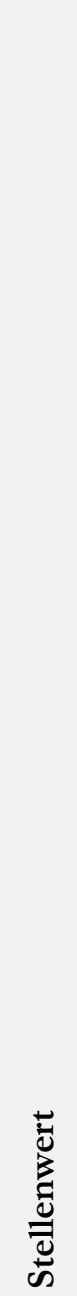 & $\begin{array}{l}\text { Relevanz für das } \\
\text { Dissertationsprojekt }\end{array}$ & $\begin{array}{l}\text { Diese Arbeit nimmt im Rahmen meines Vorhabens die Funktion } \\
\text { eines Grundlagen-skizzierenden Elements ein und stellt in der } \\
\text { dahinterliegenden Logik den ersten Schritt dar: Das } \\
\text { Offensichtliche beleuchten. Nicht nur werden lerntheoretische } \\
\text { Grundannahmen hinterfragt, sondern auch ein Feld skizziert, in dem } \\
\text { die Begegnung mit dem Anderen in der akademischen Lehre bereits } \\
\text { thematisiert wird. } \\
\text { In Kontexten internationaler Hochschullehre begegnen Lehrende } \\
\text { wie Studierende mitunter sich widersprechende Erwartungen an das } \\
\text { Handeln in ihrer Disziplin. Im Zuge meiner Forschung konnte ich } \\
\text { unterstreichen, dass die akademische Sozialisation als Teil } \\
\text { individueller Identitäten nicht nur disziplinär, sondern insbesondere } \\
\text { lokal geprägt ist. Kollidieren in Lehr-Lernsituationen } \\
\text { unterschiedliche diskursive Praktiken, werden diese durch die } \\
\text { Begegnung mit dem Anderen und die Erfahrung einer ins Stocken } \\
\text { geratenen sozialisierten Handlungsroutine sichtbar. Es konnte } \\
\text { zudem skizziert werden, dass durch Reflexionsimpulse, die ihren } \\
\text { Fokus gezielt auf diese Begegnungsprozesse und die mit ihnen } \\
\text { verbundenen Herausforderungen richten, derlei Erfahrungen für das } \\
\text { weitere Lernen genutzt werden können, insbesondere zur } \\
\text { Weiterentwicklung individueller Lehrkompetenzen und dem Ziel } \\
\text { professionell(er)en Handelns in der internationalen Lehre. } \\
\text { Im Querschnitt konnte die Relevanz der Erfahrung im Lernen sowie } \\
\text { des Lernkontextes und seiner sozialen Verortung und in der Folge } \\
\text { der Einfluss individueller Identitäten für das Handeln in } \\
\text { akademischen Lehr-Lernkontexten unterstrichen werden. }\end{array}$ \\
\hline
\end{tabular}




\section{Teilstudie B: Das Antizipierte ergründen}

Listening Out and Dealing with Otherness. A Postcolonial Approach to Higher Education Teaching

\begin{tabular}{|c|c|c|}
\hline \multicolumn{2}{|c|}{ Aspekt } & \multirow{2}{*}{$\begin{array}{l}\text { Beschreibung } \\
\text { Thielsch, A. (2019). Listening Out and Dealing with Otherness. A } \\
\text { Postcolonial Approach to Higher Education Teaching. In Arts and } \\
\text { Humanities in Higher Education. February 26, 2019. DOI: } \\
\text { 10.1177/1474022219832459. }\end{array}$} \\
\hline & $\begin{array}{l}\text { Vollständige } \\
\text { Referenz }\end{array}$ & \\
\hline & Schlagworte & $\begin{array}{l}\text { Postcolonial pedagogy, modes of knowledge production, hidden } \\
\text { curriculum, otherness }\end{array}$ \\
\hline & $\begin{array}{l}\text { Publikationsorgan } \\
\text { und Auswahl }\end{array}$ & $\begin{array}{l}\text { Das Sage Journal Arts and Humanities in Higher Education (AHH) ist } \\
\text { eine internationale Zeitschrift mit Fokus auf } \\
\text { geisteswissenschaftliche Diskurse im Hochschullehrkontext und } \\
\text { eine online first Veröffentlichung ermöglicht; die Begutachtung } \\
\text { erfolgt über ein double blind Peer Review. } \\
\text { Inhaltlich fiel die Wahl auf dieses Journal, da } 2016 \text { hier ein Beitrag } \\
\text { zur Relevanz der Dekolonisierung der Academia erschien, der ein } \\
\text { theoretischer Anker dieses interdisziplinären, nicht eindeutig zu } \\
\text { verortenden Beitrags ist. }\end{array}$ \\
\hline & Publikationsstatus & Erschienen. \\
\hline 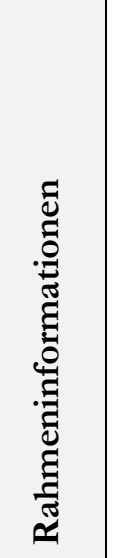 & Publikationshistorie & $\begin{array}{l}\text { Einreichung Juni 2018, Rückmeldung zur Veröffentlichung nach } \\
\text { geringer Überarbeitung Dezember 2018, Erschienen Februar } 2019 \\
\text { als open access Version. Veröffentlichung im Rahmen einer konkreten } \\
\text { Ausgabe im Laufe des Jahres. } \\
\text { Erste Version des Papers: Bewerbung zur Beteiligung mit einem } \\
\text { Kapitel im geplanten Sammelband „Decolonising University } \\
\text { Teaching and Gender“ (Routledge, London/New York), Annahme } \\
\text { Januar 2017; Einreichung des Langbeitrags Juni 2017; Information } \\
\text { zur inhaltlichen Neuausrichtung mit exklusivem Gender-Fokus des } \\
\text { Bandes und Angebot zur Umgestaltung des Beitrags sowie } \\
\text { selbstgewählte Rücknahme des Beitrags August 2017. }\end{array}$ \\
\hline 芳 & $\begin{array}{l}\text { Abstract } \\
\text { (wie veröffentlicht) }\end{array}$ & $\begin{array}{l}\text { Postcolonial pedagogy invites academic teaching staff to create } \\
\text { situations, in which hegemonic modes of knowledge production can } \\
\text { be critically reflected and one's own entanglement as disciplinary }\end{array}$ \\
\hline
\end{tabular}




\begin{tabular}{|c|c|}
\hline & $\begin{array}{l}\text { socialised member of (western) academia experienced. Such a } \\
\text { postcolonial approach has been applied to a seminar in the context } \\
\text { of cultural musicology and its impact on teaching and learning } \\
\text { analysed. } \\
\text { In this paper the findings of the accompanying research are } \\
\text { presented and discussed in relation to the concept of Bildung, } \\
\text { theories on individual learning (in higher education), and current } \\
\text { processes to internationalise the curricula. Throughout the } \\
\text { argumentation I will demonstrate how postcolonial pedagogy may } \\
\text { cause the construction of otherness and why this simultaneously } \\
\text { constitutes the biggest challenge as well as the profoundest reward } \\
\text { when applying such an approach to university teaching. In addition } \\
\text { to that, this paper introduces a definition of postcolonial pedagogy } \\
\text { and offers recommendations to foster its implementation in higher } \\
\text { education contexts. }\end{array}$ \\
\hline Erkenntnisinteresse & $\begin{array}{l}\text { Welche Herausforderungen und Chancen ergeben sich durch den } \\
\text { Einsatz eines didaktischen Ansatzes, der basierend auf den } \\
\text { Parametern postkolonialen Denkens entwickelt und erprobt wurde? } \\
\text { Was ist bei der Anwendung eines solchen Ansatzes zu beachten, um } \\
\text { sowohl die ermittelten Hürden als auch Potentiale im Sinne des } \\
\text { Bildungsgedankens nutzen zu können? }\end{array}$ \\
\hline Forschungsfeld & $\begin{array}{l}\text { Geisteswissenschaftliche Hochschullehre an einer deutschen } \\
\text { Universität, Master-Studiengang, Seminar, Lehren im Teaching } \\
\text { Team }\end{array}$ \\
\hline $\begin{array}{l}\text { Aufbau und } \\
\text { Argumentation }\end{array}$ & $\begin{array}{l}\text { Nach einer Einordnung in den größeren Kontext der Forschung } \\
\text { sowie des methodischen Vorgehens (Introducing the } \\
\text { (methodological) setting) wird im zweiten Teil das theoretische } \\
\text { Grundgerüst des papers vorgestellt (Thinking about academia, } \\
\text { the concept of Bildung, and individual learning). Hier wird } \\
\text { argumentiert, inwiefern das Ziel der Bildung im westlichen Diskurs } \\
\text { durch koloniales Gedankengut beeinflusst ist und so das Was und } \\
\text { Wie des Lernens an Hochschulen prägt. Erste Gedanken zur } \\
\text { Bedeutung individuellen Lernens, seiner sozialen Eingebundenheit } \\
\text { und der damit verknüpften Sozialisation werden skizziert, bevor im } \\
\text { nächsten Abschnitt (The case study and its context) das } \\
\text { untersuchte Fallbeispiel näher betrachtet wird. Für diese Vorstellung } \\
\text { wird der modulare Rahmen und die zu antizipierenden Einflüsse } \\
\text { verschiedener Ebenen des Curriculums analysiert und so die } \\
\text { Grundlage zur Gestaltung der Lehre vorgestellt. }\end{array}$ \\
\hline
\end{tabular}




\begin{tabular}{|c|c|}
\hline & $\begin{array}{l}\text { Im folgenden Kapitel vier (Critical reflection on a postcolonial } \\
\text { approach to teaching) wird sodann in drei Bereichen dargelegt, } \\
\text { welche - erwarteten und unerwarteten - Herausforderungen ein } \\
\text { postkolonialer Ansatz in der Lehre mit sich bringen kann und } \\
\text { wodurch ihr Entstehen jeweils theoretisch zu erklären ist. In diesem } \\
\text { Herzstück des Papers wird zum einen der Einfluss der } \\
\text { akademischen Sozialisation im Studium und zum anderen die } \\
\text { Begegnung mit dem (konstruierten) Anderen als zentrale } \\
\text { Erkenntnisse argumentiert und belegt, bevor im nächsten Bereich } \\
\text { eine Zusammenfassung der gesammelten Erfahrungen als } \\
\text { Empfehlungen für die Anwendung dieses Lehransatzes gegeben } \\
\text { wird (Recommendations for higher education teaching). } \\
\text { Da es sich hier um ein pädagogisches Vorgehen handelt, das bislang } \\
\text { keine umfassende Herleitung oder Definition erfahren hat, schließt } \\
\text { der Artikel mit dem Angebot einer solchen (Finally... a } \\
\text { definition). Begründet in einer Definition des Ursprungskonzepts } \\
\text { Postkolonialismus werden die Ziele und Grundelemente eines } \\
\text { postkolonialen Ansatzes für die Hochschullehre expliziert. }\end{array}$ \\
\hline $\begin{array}{l}\text { Methodisches } \\
\text { Vorgehen }\end{array}$ & $\begin{array}{l}\text { Qualitative Datenerhebung im Modus einer ethnographischen } \\
\text { Feldforschung; } \\
\text { Triangulation und interpretativ-inhaltsanalytisch Auswertung dreier } \\
\text { Datensätze: } \\
\text { - Leitfragengestützte Beobachtungsnotizen aus dem Feld der } \\
\text { „Seminarsitzung“, } \\
\text { - Reflexionsprotokoll-Einträge der Team Teaching-Arbeit, } \\
\text { - Angaben der Beteiligten im Rahmen eines nachträglichen } \\
\text { Reflexions-Fragebogens. }\end{array}$ \\
\hline $\begin{array}{l}\text { Zentrale } \\
\text { Erkenntnisse }\end{array}$ & $\begin{array}{l}\text { Die akademische Sozialisation der Studierenden (und Lehrenden) } \\
\text { wird in der Begegnung mit einem unbekannten Lehransatz wie dem } \\
\text { der postkolonialen Pädagogik herausgefordert und äußert sich auf } \\
\text { Ebene der Interaktion als Auswirkung widersprüchlicher } \\
\text { Erwartungen im bidden curriculum. } \\
\text { Das übergeordnete Ziel postkolonialer Pädagogik - } \\
\text { epistemologische Vielfalt zu ermöglichen und die Konstruktion } \\
\text { individuellen Wissens kritisch zu reflektieren - wird jedoch erst } \\
\text { durch eine bewusste Gestaltung dieser Begegnung mit dem Anderen } \\
\text { lernförderlich, da diese explizit auf vorhandenen } \\
\text { Wissenskonstrukten aufbaut und so den Zugang ermöglicht, um } \\
\text { bestehendes Wissen zu hinterfragen. }\end{array}$ \\
\hline
\end{tabular}




\begin{tabular}{|c|c|c|}
\hline & & $\begin{array}{l}\text { Die Lehre als Teaching Team hat sich in diesem Zusammenhang als } \\
\text { sinnvolles Instrument herausgestellt, um die eigene Involviertheit als } \\
\text { Lehrperson in eine akademische Tradition (durch Reflexion des } \\
\text { Lehrhandelns) zu erkennen. }\end{array}$ \\
\hline 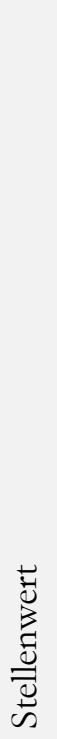 & $\begin{array}{l}\text { Relevanz für das } \\
\text { Dissertationsprojekt }\end{array}$ & $\begin{array}{l}\text { Die hier durchgeführte Lehrforschung stellt im Rahmen der } \\
\text { Dissertation den zweiten logischen Schritt der } \\
\text { Erkenntnisgewinnung dar: Das Antizipierte ergründen. Die } \\
\text { Realisierung eines Lehransatzes zu erforschen, der für Lehrende } \\
\text { und Lernende gleichermaßen herausfordernd ist, und dies aus der } \\
\text { Perspektive einer Involvierten im Feld zu tun, hat neue } \\
\text { Erkenntnisse über das lernförderliche Potential der Begegnung mit } \\
\text { dem Anderen ermöglicht. Auf Basis dieses induktiven Vorgehens } \\
\text { konnte nicht nur die Relevanz der akademischen Sozialisation } \\
\text { bekräftigt werden, sondern auch der Einfluss jener Mechanismen, } \\
\text { die im Prozess individueller Identitätskonstruktion zutage treten. } \\
\text { Erstmals für das Dissertationsprojekt findet das Konzept der } \\
\text { Alterität implizit Anwendung und hilft zu erklären, wodurch die } \\
\text { aufgetretenen Herausforderungen begründet sind und welches } \\
\text { Potential sie beinhalten. }\end{array}$ \\
\hline
\end{tabular}




\section{Teilstudie C: Das Erwartete überprüfen}

Feedback in Team Teaching-Konstellationen. Eine Studie zur Lehrkompetenzentwicklung durch Begegnung anderer Wissensformen

\begin{tabular}{|c|c|c|}
\hline \multicolumn{2}{|c|}{ Aspekt } & Beschreibung \\
\hline \multirow{5}{*}{ 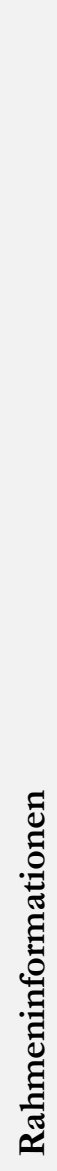 } & $\begin{array}{l}\text { Vollständige } \\
\text { Referenz }\end{array}$ & $\begin{array}{l}\text { Thielsch, A. (2019). Feedback in Team Teaching-Konstellationen. } \\
\text { Eine Studie zur Lehrkompetenzentwicklung durch Begegnung } \\
\text { anderer Wissensformen. In die hochschullehre, 5, 161-186. }\end{array}$ \\
\hline & Schlagworte & $\begin{array}{l}\text { Team Teaching, akademische Lehrkompetenzentwicklung, } \\
\text { Feedback, Begegnungen }\end{array}$ \\
\hline & $\begin{array}{l}\text { Publikationsorgan } \\
\text { und Auswahl }\end{array}$ & $\begin{array}{l}\text { die hochschullehre ist eine interdisziplinäre Open Access Zeitschrift für } \\
\text { Studium und Lehre, die in der hochschuldidaktischen Community } \\
\text { aufgrund ihres vielschichten Fokus' auf Forschungs-, Praxis- sowie } \\
\text { Praxisforschungsbeiträgen rezipiert und zur Veröffentlichung } \\
\text { herangezogen wird. Die Begutachtung ist als double blind Peer } \\
\text { Review organisiert. } \\
\text { Die Entscheidung, in dieser Zeitschrift zu veröffentlichen, erfolgte } \\
\text { sowohl aufgrund ihrer expliziten Qualitätskriterien als auch wegen } \\
\text { ihres Stellenwerts in der Community der Deutschen Gesellschaft für } \\
\text { Hochschuldidaktik (dghd). }\end{array}$ \\
\hline & Publikationsstatus & Erschienen. \\
\hline & Publikationshistorie & $\begin{array}{l}\text { Einreichung im August 2018, Rückmeldung zur Annahme mit } \\
\text { geringer Überarbeitung im Januar 2019, Veröffentlichung im } \\
\text { Februar } 2019 .\end{array}$ \\
\hline 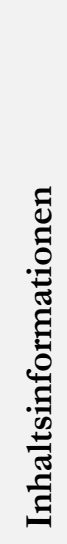 & $\begin{array}{l}\text { Abstract } \\
\text { (wie veröffentlicht) }\end{array}$ & $\begin{array}{l}\text { Das Lehren in Team Teaching-Konstellationen kann durch die darin } \\
\text { generierten Feedbackinformationen zur akademischen } \\
\text { Lehrkompetenzentwicklung beitragen. Um dies zu untersuchen, } \\
\text { wurde eine Studie zum wahrgenommenen Feedback in der als } \\
\text { Teaching Team realisierten Lehre durchgeführt und die erhobenen } \\
\text { Daten hinsichtlich verschiedener Feedbackquellen ausgewertet. Vor } \\
\text { dem Hintergrund theoretischer Er-kenntnisse zur } \\
\text { Lehrkompetenzentwicklung, der Lehre als Teaching Team sowie der } \\
\text { Ziele und Wirkungsweisen von Feedbackprozessen, werden die } \\
\text { Ergebnisse der Studie in der vorliegenden Arbeit zusammengefasst } \\
\text { und diskutiert. Die Begegnung anderer Vorstellungen über das }\end{array}$ \\
\hline
\end{tabular}




\begin{tabular}{|c|c|}
\hline & $\begin{array}{l}\text { Lehren und Lernen, so wird argumentiert, ist hierbei von } \\
\text { Bedeutung. }\end{array}$ \\
\hline Erkenntnisinteresse & $\begin{array}{l}\text { Inwiefern bieten die in Team Teaching-Konstellationen erzeugten } \\
\text { Feedback-informationen Lehrenden unterschiedlicher } \\
\text { Lehrerfahrungen (grundsätzlich) Anlass zur akademischen } \\
\text { Lehrkompetenzentwicklung? }\end{array}$ \\
\hline Forschungsfeld & $\begin{array}{l}\text { Interdisziplinäre Lehrende, die in einem universitären Kontext und } \\
\text { in Verbindung zu einem hochschuldidaktischen Angebot eine } \\
\text { Lehrveranstaltung als Teaching Team geplant und durchgeführt } \\
\text { haben und in sich in der Team-Zusammensetzung durch } \\
\text { unterschiedliche Grade der Lehrerfahrung auszeichneten. }\end{array}$ \\
\hline $\begin{array}{l}\text { Aufbau und } \\
\text { Argumentation }\end{array}$ & $\begin{array}{l}\text { Zu Beginn des Papers werden Verortung und Aufbau der Arbeit } \\
\text { skizziert und so der Rahmen dieser quantitativen Studie gelegt. } \\
\text { Ausgehend von einem relationalen, subjektbezogenen } \\
\text { Lernverständnis wird die Motivation der präsentierten Studie } \\
\text { eingeleitet und das Ziel vorgestellt: Die Lehre als Teaching Team } \\
\text { soll dahingehend untersucht werden, welchen Wert die dabei } \\
\text { entstehenden Feedbackinformationen für die } \\
\text { Lehrkompetenzentwicklung der beteiligten Lehrenden haben kann. } \\
\text { Ein Wert, so die Annahme, der sich in der die Begegnung anderer } \\
\text { Perspektiven und Wahrnehmungen in Lehrsituationen } \\
\text { wiederspiegelt. Die theoretische Grundannahmen werden sodann } \\
\text { für die hier behandelten Felder - akademische } \\
\text { Lehrkompetenzentwicklung, Lehre als Teaching Team und sowie } \\
\text { Relevanz von Feedback - umrissen, bevor im nächsten Teil } \\
\text { (Erkenntnisinteresse und Forschungsdesign) der methodische } \\
\text { und soziale Kontext der hier vorgestellten Studie expliziert wird. } \\
\text { Neben Informationen zur Entwicklung des Instruments, der } \\
\text { Durchführung der Erhebung sowie ihrer Rahmenbedingungen } \\
\text { (Stichprobe, Rücklauf etc.), werden die zu prüfenden Hypothesen } \\
\text { dargelegt und das übergeordnete Erkenntnisinteresse begründet. } \\
\text { Den Kern der Arbeit stellt das vierte Kapitel dar, in dem schließlich } \\
\text { Ergebnisse der Erhebung präsentiert werden. Eingangs wird } \\
\text { erläutert, weshalb die aufgestellten Hypothesen (dass die } \\
\text { Teilnehmenden die eigene Lehrkompetenzentwicklung als Ziel } \\
\text { hatten, dieses Ziel durch die Lehre als Teaching Team theoretisch } \\
\text { erreichen konnten, dass das wahrgenommene Feedback durch dieses } \\
\text { Lehren sowie durch die Lehrerfahrung der Teilnehmenden } \\
\text { beeinflusst wurde) anhand der gewonnenen Daten gestützt werden } \\
\text { können. Anschließend erfolgt eine vertiefte Darstellung der }\end{array}$ \\
\hline
\end{tabular}




\begin{tabular}{|c|c|}
\hline & $\begin{array}{l}\text { Ergebnisse in Relation zu den Team Teaching-inhärenten } \\
\text { Feedbackquellen: den Team Teaching-Mitgliedern, den Studierenden } \\
\text { sowie den initiierten Selbstreflexionsprozessen. Das gemeinsame } \\
\text { Lehren und die hier ermöglichte Begegnung anderer Wissensformen } \\
\text { und Handlungslogiken, so kann nunmehr statistisch belegt } \\
\text { argumentiert werden, stellen Feedbackinformationen bereit, die die } \\
\text { Lehrkompetenzentwicklung einer Person zu adressieren vermögen. } \\
\text { Im nächsten Teil der Arbeit, der Diskussion und } \\
\text { Zusammenführung, werden die vielschichtigen Ergebnisse } \\
\text { komprimiert und davon Implikationen für die Hochschullehre } \\
\text { allgemein und die Lehre als Teaching Team insbesondere abgleitet. } \\
\text { Zum Ende des Artikels lädt der Ausblick dazu ein, an diese Studie } \\
\text { anschließende Forschungsfragen zu bedenken, bevor die } \\
\text { Abschließende Bemerkungen hervorheben, dass die Erforschung } \\
\text { von Feedbackprozessen ein Instrument sein kann, um } \\
\text { subjektbezogene Lernzugänge und die Bedeutung des sozialen } \\
\text { Kontextes im Lernen eingehender zu beleuchten. }\end{array}$ \\
\hline $\begin{array}{l}\text { Methodisches } \\
\text { Vorgehen }\end{array}$ & $\begin{array}{l}\text { Quantitative Fragebogenstudie bei Teilnehmenden eines } \\
\text { hochschuldidaktischen Team Teaching-Programms zwischen SoSe } \\
2010 \text { und WiSe 2017/2018; } \\
\text { Fragebogenteile: } \\
\text { - Angaben zu Person und Teilnahmekontext, } \\
\text { - Angaben zu Zielen und Motivation der Teilnahme am Team } \\
\text { Teaching-Programm, } \\
\text { - Angaben zur Realisierung des Team Teaching, } \\
\text { - Wahrgenommenes Feedback im Lehren als Teaching Team; } \\
\text { Statistische Auswertung der Daten mittels des offenen, SPSS- } \\
\text { kompatiblen Programms PSPP. }\end{array}$ \\
\hline $\begin{array}{l}\text { Zentrale } \\
\text { Erkenntnisse }\end{array}$ & $\begin{array}{l}\text { Inhaltlich zeichnen sich zwei übergeordnete Erkenntnisse ab: } \\
\text { - Die in der Lehre als Teaching Team entstehenden } \\
\text { Feedbackinformationen können die } \\
\text { Lehrkompetenzentwicklung der beteiligten Lehrenden } \\
\text { fördern. } \\
\text { - Die Begegnung anderer Perspektiven und Wissenslogiken in } \\
\text { Team Teaching-Konstellationen zeichnen sich als Impuls } \\
\text { für diesen lehrkompetenzförderlichen Aspekt ab. } \\
\text { Zudem konnte auf methodischer Ebene das neu entstandene } \\
\text { Instrument zur Erfassung der Lehrkompetenzentwicklung } \\
\text { erfolgreich pilotiert werden. }\end{array}$ \\
\hline
\end{tabular}




\begin{tabular}{|c|c|c|}
\hline 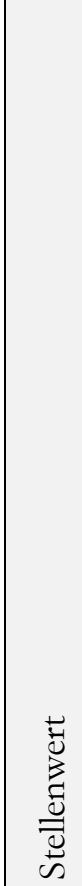 & $\begin{array}{l}\text { Relevanz für das } \\
\text { Dissertationsprojekt }\end{array}$ & $\begin{array}{l}\text { Nachdem in den ersten beiden Arbeiten des Dissertationsprojektes } \\
\text { das Potential des Alterität-Konzeptes in akademischen Lehr- } \\
\text { Lernkontexten begründet und seine Qualität im Verstehen } \\
\text { lernförderlicher Aspekte in Begegnungsprozessen erfasst werden } \\
\text { konnte, wurde diese dritte Studie explizit dafür designed, den } \\
\text { Mehrwert einer solchen Begegnung mit dem Anderen auch } \\
\text { statistisch zu begründen. Mit dem übergeordneten Ziel, das } \\
\text { Erwartete zu überprüfen, wurde das Erhebungsinstrument } \\
\text { gestaltet und Items entwickelt, die die Wahrnehmung von } \\
\text { Feedbackinformationen durch das erlebte Andere erfassen sollten. } \\
\text { Mit Blick auf das Dissertationsprojekt ist durch diese Arbeit der } \\
\text { Prozess wissenschaftlicher Erkenntnisgewinnung nun komplettiert: } \\
\text { Die Vermutung, dass im Hochschulkontext dem Anderen begegnet } \\
\text { wird und diese Begegnung Einfluss auf das Lernen und Lehren hat, } \\
\text { konnte in Studie A näher beleuchtet werden, bevor in Studie B } \\
\text { deren Hintergründe und Qualitäten erfasst wurden, um hier - in } \\
\text { Studie C - auf dieser Vorarbeit begründete Hypothesen zum } \\
\text { Potential von Begegnungsprozesse mit dem Anderen zu überprüfen. }\end{array}$ \\
\hline
\end{tabular}




\section{Anhang: Veröffentlichte Artikel}

Thielsch, A. (2017). Approaching the Invisible: Hidden Curriculum and Implicit

Expectations in Higher Education. In Zeitschrift für Hochschulentwicklung, 12(4), 167-187. DOI:10.3217/zfhe-12-04/11.

Thielsch, A. (2019). Listening Out and Dealing with Otherness. A Postcolonial Approach to Higher Education Teaching. In Arts and Humanities in Higher Education. February 26, 2019; DOI: 10.1177/1474022219832459.

Thielsch, A. (2019). Feedback in Team Teaching-Konstellationen. Eine Studie zur Lehrkompetenzentwicklung durch Begegnung anderer Wissensformen. In die bochschullehre, 5, 161-186. 


\title{
Angelika THIELSCH${ }^{1}$ (Göttingen)
}

\section{Approaching the Invisible: Hidden Curriculum and Implicit Expectations in Higher Education}

\begin{abstract}
In situations of colliding discursive practices, their respective qualities can emerge. Based on this assumption, a research project has been conducted at the University of Göttingen that aimed at better understanding the hidden curriculum experienced by former exchange students. Using critical discourse analysis and an inductive approach as foundation, statements of this group have been collected and analysed. Implications regarding the experienced hidden curriculum were used to develop a reflective guideline for academic teaching staff. This paper introduces the theoretical and methodological foundation of my research and offers insight in the findings on how to approach challenges caused by the implicit nature of the hidden curriculum.
\end{abstract}

\section{Keywords}

Hidden curriculum in higher education, individual learning (and) experience, exchange students, critical discourse analysis

1 email: angelika.thielsch@zvw.uni-goettingen.de

\section{(cc) BY-NC-ND}

Scientific Contribution 


\section{Introduction}

In higher education, students and teachers alike sometimes struggle with the implicit expectations of each other. These expectations - whether how to interact in a lecture or how to prepare for an exam - may cause confusion and eventually obstacles for both. During the last decades, the idea of hidden aspects in university teaching is being discussed from different perspectives (COTTON, WINTER \& BAILEY, 2013; SAMBELL \& McDOWELL, 1998). After having realised that in each university curriculum one can find implicit levels which exist alongside the explicit ones, the focus has shifted to a more global perspective. Today, our understanding of diverse layer of the curriculum is being used to decolonise academia and hegemonic ideas of knowledge production (LE GRANGE, 2016) as well as to find reasonable ways to internationalise universities (LEASK, 2015; KILLICK, 2015). It is in this context of the internationalisation of curricula that my research is located.

LEASK $(2015$, p. 8) defines the hidden curriculum as "[...] the various unintended, implicit and hidden messages sent to students [...]" by numerous aspects in teaching and learning. Each curriculum consists of three interactive elements - the formal, the informal, and the hidden curriculum. ${ }^{2}$ Students experience these different elements " $[\ldots]$ as a dynamic interplay of teaching and learning processes, content, and activities in and out of the classroom" (LEASK, 2015, p. 9). Within the mixture of the three, the hidden curriculum is the least visible, yet it is supposed to be lived and (re)produced within the formal as well as the informal curriculum. For example, "[...] the social rituals which govern where to sit within a classroom space, how to enact the student-master relationship, or where cooperation ends and

${ }^{2}$ LEASK $(2015$, p. 8) defines the formal curriculum as "[...] the syllabus as well as the orderly, planned schedule of experiences and activities that students must undertake as part of their degree program" and the informal curriculum as "[...] the various support services and additional activities and options organised by the university that are not assessed [...]." 
collusion, actually, begins" (KILLICK, 2015, p. 84), are labelled as part of the hidden curriculum. These social rituals need to be experienced and understood from within, as socialised members of one specific higher education setting. The various elements of a hidden curriculum and its core is closely linked to the local context in which it is lived by academic and non-academic staff as well as by those students (already) familiar to it. In consequence, it most likely shows itself in situations of contradictory expectations regarding social behaviour and approaches in teaching and learning.

The motivation for my research derived from these fundamental thoughts. I wondered how the hidden curriculum of one local academic context might be exposed. Even though I did not assume that it could be clearly defined, I expected to detect broader areas in which it tends to emerge. Therefore, I chose to collect experiences of a group of students that most likely could yield insights: Exchange students. They might, due to their prior academic socialisation and their limited stay at the University of Göttingen, still be aware of contradictory expectations in terms of teaching and learning practices. In this paper, I present the theoretical and methodological fundament of my research conducted at the Georg-August-University of Göttingen in spring 2017. I offer insight into the data collected and demonstrate how - based on my findings - I developed a reflective guideline for academic teaching staff. A guideline, which aims at supporting teachers to become aware of their own involvement in (re)producing elements of the hidden curriculum in one local academic context. Educational staff development is said to be a key factor to successfully internationalise curricula in general and especially when it comes to applying the concept of Internationalisation at Home ${ }^{3}$ (BEELEN \& JONES, 2015, p. 70). The outcome of this project fully aligns with this notion.

${ }^{3}$ BEELEN \& JONES (2015, p. 69) define Internationalisation at Home as follows: "Internationalization at Home is the purposeful integration of international and intercultural dimensions into the formal and informal curriculum for all students within domestic learning environments." 
In my research, I do not seek to further depict aspects challenging for international students nor strengthen any discussion on which cultural background may or may not lead to specific expectations in international higher education contexts. Instead, I aim at finding a way to support faculty to better understand their own expectations, its individual origins and relevance, detecting implicit aspects in their teaching and thereby enabling them to henceforth make them explicit ${ }^{4}$. Especially for international students such transparency is crucial, as they are studying in an academic system which is unfamiliar to them (CARROLL, 2015, p. 21). Offering them support and guidance to better understand teaching and learning settings unfamiliar to them and their learning in a new context (compared to their home universities), can be seen as one responsibility of academic staff (ibid.). On a broader level I argue that being explicit in one's teaching is relevant in all such situations; when dealing with international students as much as when facilitating interdisciplinary courses or when teaching not-yet-academically-socialised first year students. Hence, the research presented in this paper aims at providing a tool for academic staff that helps them to better understand their own expectations in teaching, to make these explicit, and by doing so supports them in shouldering the responsibility mentioned above.

\section{On Academic Learning (and) Discourses}

Individual experiences constitute the core of the findings presented in this paper: Experiences made by exchange students during their stay at the University of Göttingen, collected by an online survey, as well as experiences reflected by teachers retrospectively, using the developed guideline to detect aspects of the hidden curriculum in their own teaching. In this section I will explore why focussing on indi-

\footnotetext{
${ }^{4}$ My approach has been influenced by the work of Jude CARROLL (2015) and the emphasis she puts on being explicit when teaching in international higher education settings.
} 
vidual experiences ${ }^{5}$ offers a suitable starting point to uncover something that is supposedly hidden, and briefly reflect my approach from the point of view of critical discourse analysis. To do so, I will start by stressing the relevance of experiences for processes of learning and competence development in general.

Learning, especially adult learning, can be defined as the process of building new knowledge based on and linked to existing knowledge (c.f. BLUMBERG, 2009). Each learning process depends on multiple components that can be narrowed down to three aspects: A person needs some kind of abstract information, some practical experience in relation to it, and some reflective awareness connecting both (FINK, 2013). In order to create new meaning, a person connects new ideas to knowledge s/he already has. Knowledge on the content-level, but also knowledge on the level of knowledge production. That means that within a person's prior knowledge one can find traces of learning biographies and a person's (learning) socialisation. This learning socialisation is - like all processes of socialisation - multi-layered and continually developing. A persons' learning experiences are shaped and influenced by formal learning as well as informal learning settings, all of which are located in specific contexts and filled with meaning by the people belonging to it. Assuming that "[p]eople do not make meaning just as individuals" (GEE \& HANDFORD, 2014 , p. 5), but as members of social groups, this means for academic contexts that each new learning experience increases a student's knowledge on teaching and learning in higher education as well as his or her socialisation as a member of one specific academic discourse.

A hidden curriculum is part of such a locally embedded academic discourse. People who are socialised within this particular context share the same ideas on how teaching and learning in higher education work. Whereas these ideas don't need to be named and made explicit to members of the same discourse, anyone unfamiliar

${ }^{5}$ KOLB (1984, p. 38) emphasised the importance of experience early by stating that "Learning is the process whereby knowledge is created through the transformation of experience." 
with its discursive practice is likely to be confused. This confusion is the basis I used in my research. Since it is a human need to make meaning (FINK, 2013, p. 117) a person will most likely seek to better understand why something did not work as expected, and in consequence remember situations in which s/he experienced (challenging) differences. Here, I used this assumption in combination with the above illustrated understanding of learning processes to collect and analyse social practices ${ }^{6}$ in academic teaching. I developed a survey for exchange students, in which I asked them to describe situations, practices, and ways of communication expected (and repeatedly experienced before), but not made during their stay as exchange students. Thereby I aspired to address two layers of social discursive practices: The ones expected as well as the ones experienced. Based on the findings of this survey, I designed a reflective guideline for academic teaching staff, in which they were asked to reflect on those aspects that seemed to be most challenging for the exchange students who were interviewed.

Both instruments that evolved during my research can be grounded in the abovementioned aspects of individual learning: abstract information, practical experience and reflective awareness. As illustrated in figure 1, the survey invited exchange students to reflect on experienced challenges in order to name (and eventually better understand) the abstract ideas underneath. Based on the same principle of individual learning the reflective guideline is designed. Here, academic teaching staff is invited to reflect on their ideas and understandings of teaching and learning in higher education, to remember experienced contradictory expectations and thus become (more) aware of them in future teaching contexts.

By adding the element of reflection, both instruments actively foster the connection between practical experience and abstract information, thereby initiate a better understanding of the various expectations inside a classroom and eventually allow people to observe and detect traces of the hidden curriculum more easily.

${ }^{6}$ FAIRCLOUGH (2003, p. 205) defines social practice as "[...] relatively stabilized form of social activity." 
Online Survey [Exchange Students' perspective]

Reflective Guideline

[Teachers' perspective]
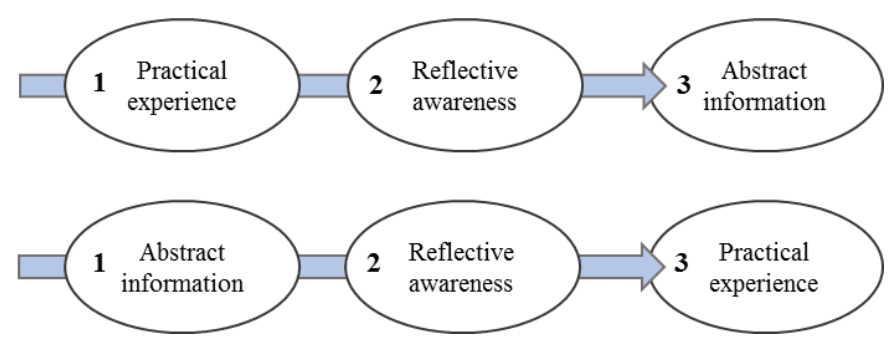

Figure 1: Developed Instruments and Their Value for Individual Learning Processes

Let me emphasise that in this project I did not purely aim at collecting data that could yield insights into discursive practices, but sought to enhance critical understanding and discourse awareness with the people involved: the participating exchange students and - first and foremost - the academic teaching staff working with the reflective guideline in the future. An endeavour that originates in the socalled critical discourse analysis (henceforth CDA).

CDA belongs to the wider field of discourse analysis and can be classified as critical social research. It focusses on the dialectical relationship between discourse on the one hand and its elements of social practice on the other (FAIRCLOUGH, 2003 , p. 202ff.). Such social practices are realised by linguistic expressions, which - by using them - reproduce the discourse they originate from. Critical discourse analysis focusses at this connection. It examines how social practice is being verbalised, how individuals describe their experiences, and how this influences the respective discourse. Based on the notion that discourses simultaneously shape the world and are shaped by it, CDA consciously locates itself within a discourse. It tries to affect it by making inherent (power) structures visible and in consequence easier to question. The intention to intervene and to have an impact on social practices of one discursive reality is inherent to CDA and needs to be made explicit (KELLER 2011, p. 29f.). 


\section{Tracking Down the Hidden Curriculum}

\subsection{The Survey}

The online survey used in this project was send to 480 individuals, all of which started their time as exchange students at the University of Göttingen in 2016. By selecting a fixed period of time, I ensured that experiences made were caused by a similar 'version' of a hidden curriculum, and therefore collected synchronic discourse information. Via email ${ }^{7}$ the closed target group was invited to share memorable experiences with teaching and learning settings as well as information on their individual background to support my research. By offering as much transparency as possible I intended to motivate sufficient people to participate, knowing that my approach and the questions in the survey itself could be challenging. Furthermore, and respecting the CDA approach, I explained my intentions (to detect traces of a hidden curriculum at the University of Göttingen and to support faculty to become aware of them).

The survey consists of two parts. Items in the first part asked the participating individuals to share information regarding their social and academic background ${ }^{8}$; items in the second - the main part - regarding their experiences in one specific discursive area: the teaching and learning settings they encountered during their stay. In an introductory text I asked the students to remember ways to communicate and interact with teachers and peers in lectures and seminars, as well as regulations

${ }^{7}$ Acknowledgment: I would like to express my gratitude to the colleagues of Göttingen International who provided the list of exchange students and send the survey information to them, thereby ensuring privacy of the target group.

${ }^{8}$ I gathered information on the duration of the stay at the University of Göttingen, a person's prior academic socialisation context, which degree s/he aspired, information regarding a person's non-academic learning biography, age, and gender. 
and scientific values they encountered. Thereafter, the following three open questions invited them to share their experiences:

- What was similar and what was different compared to the way you are (or were) used to studying at your home university?

- What was challenging for you (and/or your fellow exchange students) and why?

- If you were asked to give future exchange students any advice regarding the teaching and learning at the University of Göttingen: Which advice would you give... and why?

Each question used a different trigger to support reflective processes. Comparing the situation in Göttingen to so far known modes of teaching and learning in Higher education offered an impulse to describe individual experiences without labelling one as being more adequate than the other. Addressing specific challenges allowed to include divers perspective while recognised different needs. Asking for advice for future students prompted a person's insight knowledge of studying in Göttingen and thereby valued individual experience. Combined, all three were designed to foster meaningful responses.

Though reluctantly at first, nearly $10 \%$ of the 480 exchange students participated within the two weeks the survey was open. Experiences of 42 individuals $^{9}$ with 27 national academic socialisation backgrounds and at least ${ }^{10}$ ten different disciplinary affiliations constitute the data set my findings derived of.

${ }^{9}$ Statistics: female: 28 , male: 14 , no one refused to be labelled a fixed gender; aspiring a Bachelor's degree: 25, a Master's degree: 10, a PhD: 5, two people didn't offer any specification; 20 people started their learning biography in Europe, 13 in Asia, five on the American continents (north \& south), two in Africa, two didn't specify; 44 individuals responded to the survey, two of which I excluded because of incomplete data sets.

${ }^{10}$ Seven people did not specify their disciplinary affiliation. 


\subsection{Analysing the Data}

Using content analysis according to MAYRING (2014, p. 79-81) and his proposed steps to inductively ${ }^{11}$ develop analytic categories, I approached the data. Based on the above given understanding of learning and teaching I structured the content of each statement and extracted codes out of the material. During this process of analysing and structuring the material, I formatively as well as summatively checked the identified categories to secure their reliability. ${ }^{12}$ By doing so, I developed a set of categories that covers all statements and can be grouped in three broader areas with differing significance for this research project:

- Statements focusing on the importance of language skills

- Statements focusing on individual needs beyond academic contexts

- Statements expressing a person's academic socialisation

Whereas the first two category areas address side effects of staying abroad in general and therefore might be meaningful for everybody, the latter explicitly contains statements regarding experiences of studying abroad and thus most likely address elements of a perceived hidden curriculum as well. Within this category, I found two sub-categories and various codes shaping them, either focusing on perceived habits and customs in academia or the norms and values, which create concepts of knowledge production underlying the former. Table 1 offers an overview of all categories and codes used to analyse the data, respective anchor examples, and the number of their appearance within the data.

11 To work inductively here means that the content of the data has been used as starting point to develop analytic categories. Hence, the categories derive in close connection to the material itself and are not fixed from the beginning (as is the case in deductive content analysis).

${ }^{12}$ MAYRING (ibid.) proposes to include the first (formative) reliability check after structuring about $10-50 \%$ of the data and to use a final (summative) reliability check after coding everything. 


\section{Table 1: Analytic Categories, Anchor Examples, and Appearances}

\begin{tabular}{|c|c|c|}
\hline Classification & \multicolumn{2}{|c|}{ Experiences of Staying Abroad } \\
\hline \multirow[t]{3}{*}{ Category } & \multicolumn{2}{|c|}{ Language Skills (overall appearance $=46$ ) } \\
\hline & Focus & Anchor example \\
\hline & $\begin{array}{l}\text { Importance of language } \\
\text { skills }\end{array}$ & $\begin{array}{l}\text { Train your foreign language skills as much as possible before your } \\
\text { exchange study to avoid wasting time for adaptation (data set } 5 \text {, } \\
\text { reflective trigger advice) }\end{array}$ \\
\hline \multirow[t]{3}{*}{ Category } & \multicolumn{2}{|c|}{ Needs beyond Academic Contexts (overall appearance $=29$ ) } \\
\hline & Focus & Anchor example \\
\hline & $\begin{array}{l}\text { Individual needs beyond } \\
\text { academic contexts }\end{array}$ & $\begin{array}{l}\text { It is hard to manage time well. Because it is lonely for exchange } \\
\text { student to study in Germany. When I feel lonely, I cannot focus on } \\
\text { my study and I may feel a little bit sad (data set } 30 \text {, reflective trigger } \\
\text { challenge) }\end{array}$ \\
\hline
\end{tabular}




\begin{tabular}{|c|c|c|}
\hline Classification & \multicolumn{2}{|c|}{ Experiences of Studying Abroad } \\
\hline Category & \multicolumn{2}{|c|}{ Individual Academic Socialisation (overall appearance = 197) } \\
\hline Subcategory & \multicolumn{2}{|c|}{ Habits \& Customs in Teaching and Learning (appearance = 123) } \\
\hline \multirow[t]{4}{*}{ Codes } & Focus & Anchor example \\
\hline & $\begin{array}{l}\text { Study program organisa- } \\
\text { tion and infrastructure } \\
\text { (appearance }=44 \text { ) }\end{array}$ & $\begin{array}{l}\text { the academic structu[r]e is different from the way it is built at my } \\
\text { university with this system of credit points, I can say it is a way } \\
\text { mor[e] flexible here !! (data set } 2 \text {, reflective trigger comparison) }\end{array}$ \\
\hline & $\begin{array}{l}\text { Modes and regulations of } \\
\text { assessments (appearance } \\
=25 \text { ) }\end{array}$ & $\begin{array}{l}\text { We have min 2-3 exams per semester per subject and here only } 1 \\
\text { big exam at the end (data set } 21 \text {, reflective trigger comparison) }\end{array}$ \\
\hline & $\begin{array}{l}\text { Teacher-student- } \\
\text { interaction (appearance }= \\
\text { 54) }\end{array}$ & $\begin{array}{l}\text { The professors ask a lot of questions in class which make you to } \\
\text { prepare ahead (data set 11, reflective trigger comparison) }\end{array}$ \\
\hline Subcategory & \multicolumn{2}{|c|}{ Norms, Values and Concepts of Knowledge Production $($ appearance $=74)$} \\
\hline \multirow[t]{3}{*}{ Codes } & Focus & Anchor example \\
\hline & $\begin{array}{l}\text { Lived epistemologies } \\
\text { (appearance }=21)\end{array}$ & $\begin{array}{l}\text { difference: at the University of Goettingen we have more discussion } \\
\text { in classes, teachers make more effort [t]o guide the students to get } \\
\text { the answers themselves, not just tell them the answers directly and } \\
\text { more alternatives instead of one single 'correct answer' are accepted } \\
\text { (data set } 9 \text {, reflective trigger comparison) }\end{array}$ \\
\hline & $\begin{array}{l}\text { Importance of individual } \\
\text { learning (appearance }= \\
\text { 53) }\end{array}$ & $\begin{array}{l}\text { Teachers have more homeworks [sic] for us (data set 20, reflective } \\
\text { trigger comparison) }\end{array}$ \\
\hline
\end{tabular}

Furthermore, all types of reflective triggers (comparison, challenge, advice) proved to be successful. Even though the item that invited comparisons between expected and experienced social discursive practices offered most specific information. 


\subsection{Glimpsing at the Findings}

In this section, I will offer a comprehensive summary of my findings. Therefore, single statements ${ }^{13}$ will be used to illustrate the essence of each (sub)category. The statements will be referred to by naming the context of the person's prior academic socialisation (pas), the number of the corresponding data set (ds) as well as the reflective trigger $(\mathrm{rt})$. Since statements regarding the challenges of staying abroad in general as well as on the importance of language skills to adapt well in unfamiliar learning settings offer no new insights on how a hidden curriculum evolves, further detail on these categories will be left out here. Rather, I focus on giving a summary of the statements that have been analysed as expressing a person's academic socialisation.

A person's local academic socialisation has a traceable influence on her or his expectations regarding teaching and learning. I will elucidate this by offering some examples. First regarding the experienced concepts of teaching and learning at the University of Göttingen (its organisation and forms of assessment as well as the lived interaction in a course), second illustrating perceived concepts of knowledge production (the underlying epistemologies as well as the importance of individual learning).

Since assessment is a core element in educational systems, differences regarding its structure are crucial for students. Hence, any unexpected experiences with their examination were noteworthy for the exchange students. Whether focusing on the complexity of an exam, its format or timing, various differences between assessments at the University of Göttingen and the respective home universities can be found in the data:

13 Here, I chose not to indicate each mistake in the statements, because they demonstrate that for some exchange students, answering the questions in this survey in English was challenging as well. I merely added obviously missing letters to improve the reading experience and offered translations of German expressions. 
"To have 5 courses at the same time and all tests at the same week" (ds 22, pas Sweden, rt challenge).

“There is no 'Hausarbeit' [term paper] in France, but more 'Klausur' [written exam], all in the same time. Exams seems to be better spread in time in Göttingen" (ds 33, pas France, rt comparison).

However challenging, the explicit regulation of assessments make them comprehensible (although not easier) for exchange students. They are not essentially classified as hidden curriculum, but usually being labelled as part of the formal curriculum (CARROLL, 2015; LEASK, 2015). Still, the number of statements referring to challenging experiences with assessments indicate that they offer a sensible gateway to discuss underlying aspects of higher education teaching in one specific context. Especially, since the modes and designs of assessments implicitly originate from those concepts of knowledge production, which are discourse-specific and thus tend to differ, as we will see below.

Further traces of learning behaviour expected but hidden can be found in statements regarding the various course settings, hence organisational aspects of the formal curriculum. Statements especially mention challenges, which derived because of unfamiliar teaching formats or unexpected ways of interacting in a course.

"[...] in Germany there is a difference between 'Seminar' [seminar | discursive teaching format] and 'Vorlesung' [lecture]; I only had Vorlesungen in $\mathrm{m}[\mathrm{y}]$ life and that was quite strange at the beginning, because in 'Seminar' students are supposed to participate and express their opinions and I was not used to that kind of approach" (ds 31, pas Italy, rt comparison).

"[...] the seminars are different. The communication with teachers in Los Andes is closer that the one with the teachers in Gö. Lectures are kind of the same" (ds 37, pas Colombia, rt comparison).

Even if the teaching formats and the ways of interacting with teachers and peers are familiar, smaller customs may differ, as the following quote shows: 
"The form of lectures and seminars was similar to my home university. There were some small differences. We don't knock on the table after the lecture :)" (ds 6, pas Estonia, rt comparison).

All collected statements on how teaching and learning is organised, structured and conducted, offer valuable information on which aspects academic teaching staff might make more explicit. However, those statements which seek to elaborate on these experiences offer deeper insights; especially regarding values and norms perceived by discourse outsiders.

As mentioned in table 1, during the analysis various statements that either focus on individual learning or on the perceived epistemologies in general could be detected. Regarding the latter, I found statements that put an emphasis on the value of critical thinking and multiple perspectives, as the following examples show:

" [...] teachers make more effort [t]o guide the students to get the answers themselves, not just tell them the answers directly and more alternatives instead of one single 'correct answer' are accepted"

(ds 9, pas China, rt comparison).

In France we write much more than in Germany. Here we speak a lot all together and debate" (ds 38, pas France, rt comparison).

How different such an understanding might be compared to a person's former academic socialisation, illustrates the next statement:

"Prepare to [...] more independence and less co-dependency when it comes to learning and a wonderful opportunity to speak your mind without any inhibitions. It's going to be a marvellous experience :)"

(ds 12, pas India, rt advice).

Intertwined with the concept of knowledge production experienced at the University of Göttingen is the importance of individual learning. Sometimes this was underlined by the opportunity to choose modules independently, sometimes by stressing the amount and quality of self-study: 
"The main difference between university of Goettingen and my home university is opportunity to choose courses on your own. In my university we have fixed study schedule and strict control over the attendance" (ds 5, pas Russia, rt comparison).

"I found the self-guided nature of courses challenging, but a good skill to learn" (ds 26, pas Canada, rt challenge).

Based on these revealing, at times contradictory statements and the categories deriving from such data, significant aspects shaping the hidden curriculum become visible. Even though each single information shared by the participating students is valuable and will be analysed further, let me again emphasise the main purpose of this project: to develop an instrument to help academic teaching staff to uncover traces of this experienced hidden curriculum in their teaching. To do so, the (sub)categories illustrated above regarding a person's academic socialisation constitute a valid starting point.

\section{$4 \quad$ Making Expectations Explicit}

The reflective guideline ${ }^{14}$ designed within this project contains nine leading questions. Starting with two questions addressing a person's (disciplinary and locally influenced) understanding of knowledge production, it then offers four questions regarding its relevance in teaching and learning settings. The subsequent two questions address experienced situations, in which one's expectations collided with those of one's students. Finally, with the last question, the person is asked to choose selected aspects of her or his reflection to be applied to future teaching.

As depicted above, this guideline aims at connecting practical experience and abstract information on the process level, whereas on the content level it seeks to

14 The reflective guideline can be downloaded as Open Educational Resource via http://teachingcolours.com/download.html. 
address aspects experienced as challenging and/or implicit to exchange students. Besides referring to the same discursive practice - teaching and learning settings in higher education - the items of the guideline are complementary to those in the online survey for exchange students, while addressing the various (sub)categories regarding a person's academic socialisation.

The guideline has been tested by academic teaching staff of the University of Göttingen $^{15}$ to evaluate the comprehensibility and anticipated direction of each question. Part of this quality assessment was to analyse the reflective statements deductively, trying to detect whether the anticipated (sub)categories had been successfully addressed. This evaluation clarified that two questions needed minor adjustment to improve their comprehensibility (e.g. clarifying that by assessment I mean different forms of examination instead ways to evaluate one's teaching). The guideline now can be used to initiate reflective processes on the values and expectation influencing a person's teaching and to become more aware of one's own involvement in (re)producing elements of an experienced hidden curriculum. All the same, it may foster understanding of the obstacles caused by such a hidden curriculum and enable faculty to address these more easily if not prevent them at all.

To better understand the character of the reflective guideline and how it may initiate reflective processes of academic teaching staff, the following table illustrates responses used as anchor examples for three of the nine guiding questions; data I collected during the quality assessment.

${ }^{15}$ The guideline has been tested anonymously by nine people (five female, four male) from different disciplines, all actively teaching in 2016/2017 at the University of Göttingen. Amongst the group I invited two people without experiences with international classrooms to evaluate whether the reflective guideline works as well in other settings (e.g. awareness regarding interdisciplinary teaching). 
Table 2: Extract of the Reflective Guideline and How it Works

\begin{tabular}{|c|c|c|}
\hline Reflective question & Anchor example (data set number) & Detected subcategories \\
\hline $\begin{array}{l}\text { (3) What kind of learning } \\
\text { activities do you expect } \\
\text { from your students in the } \\
\text { various teaching formats } \\
\text { (e.g. lecture, seminar, lab } \\
\text { course, field trips)? }\end{array}$ & $\begin{array}{l}\text { [A]ctive participation in discussion. Will- } \\
\text { ingness to listen beyond what their ears are } \\
\text { used to. To do research for themselves } \\
\text { based on the questions we develop in class. } \\
\text { Present that research in a presentation. } \\
\text { Represent the results in a paper. (teacher } \\
\text { data set 8) }\end{array}$ & $\begin{array}{l}\text { - Lived epistemologies } \\
\text { - Importance of individual } \\
\text { learning, } \\
\text { - Modes and regulations of } \\
\text { assessments } \\
\text { - Teacher-student- } \\
\text { interaction }\end{array}$ \\
\hline $\begin{array}{l}\text { (5) Which communica- } \\
\text { tive principles constitute } \\
\text { - in your opinion - reason- } \\
\text { able and constructive } \\
\text { interactions in higher } \\
\text { education teaching con- } \\
\text { texts? }\end{array}$ & $\begin{array}{l}\text { Reflecting and thinking about a problem } \\
\text { with students, i.e. temporarily putting one- } \\
\text { self also into the role of someone that does } \\
\text { not yet understand a topic. This helps to } \\
\text { take away a certain amount of fear and } \\
\text { improves communication with students (for } \\
\text { example during classical lectures) (teacher } \\
\text { data set 1) }\end{array}$ & $\begin{array}{l}\text { - Lived epistemologies } \\
\text { - Importance of individual } \\
\text { learning, } \\
\text { - Study program organisa- } \\
\text { tion and infrastructure } \\
\text { - Teacher-student- } \\
\text { interaction }\end{array}$ \\
\hline $\begin{array}{l}\text { (9) Critically rethink your } \\
\text { reflection process: Which } \\
\text { aspects would you like to } \\
\text { keep in mind in your } \\
\text { future teaching activi- } \\
\text { ties... and why these in } \\
\text { particular? }\end{array}$ & $\begin{array}{l}\text { In future I will try to communicate proce- } \\
\text { dures in scientific knowledge production in } \\
\text { our field. It is something, you expect stu- } \\
\text { dents to know, but in fact such things are } \\
\text { hardly impleme[n]ted in the (rather practi- } \\
\text { cal) curriculum. For scientific works it is } \\
\text { essential to step back a little and understand } \\
\text { things on meta level. (teacher data set } 3 \text { ) }\end{array}$ & $\begin{array}{l}\text { - Lived epistemologies } \\
\text { - Importance of individual } \\
\text { learning } \\
\text { - Study program organisa- } \\
\text { tion and infrastructure }\end{array}$ \\
\hline
\end{tabular}

\section{Conclusion}

The first step in approaching the invisible in this project was to examine the importance of reflective processes and individual experiences when seeking to better understand elements of a hidden curriculum in general. Based on this, a survey to collect experiences by former exchange students of the University of Göttingen has 
been designed, the collected data analysed, and information on colliding expectations in higher education teaching and learning settings gathered. This information provided meaningful insights on how to sketch a reflective guideline to uncover values and implicit expectations shaping a person's teaching practice.

Since the notion of a hidden curriculum constitutes the centre of this paper, let me end with some remarks on that. I assume that there is never one hidden curriculum at one University, which could be uncovered, but always various versions of intertwined, locally and individually lived realities of what is expected. Therefore, the hidden curriculum is a highly flexible system, influenced by institutional and individual aspects alike. To determine, where a hidden curriculum ends and where a person's individual values and expectations begin, seems to be impossible. Yet, academic teaching staff can lessen the power of such implicit expectations in becoming more transparent in their own teaching. Within this flexible system of a hidden curriculum, they are located at the core of discursive practices, why addressing them and their teaching beliefs constitute a sensible starting point to approach the invisible

Moreover, such a reflective process might foster awareness for those elements of a study program's curriculum that need revision with regard to the process of internationalising the curricula. Because, after all, including a global perspective in higher education teaching and learning implies that both students and academic teaching staff are capable to understand and value the 'otherness' in a classroom. And more often than not this means to deal with contradictory expectations.

\section{References}

Beelen, J., \& Jones, E. (2015). Redefining internationalization at home. In The European Higher Education Area, pp. 59-72. Springer International Publishing.

Blumberg, P. (2009). Developing learner-centered teaching: A practical guide for faculty. San Francisco: Jossey-Bass. 
Carroll, J. (2015). Tools for Teaching in an Educationally Mobile World. London, New York: Routledge.

Cotton, D., Winter, J., \& Bailey, I. (2013). Researching the hidden curriculum: intentional and unintended messages. Journal of Geography in Higher Education, 37(2), 192-203. http://dx.doi.org/10.1080/03098265.2012.733684

Fairclough, N. (2003). Analysing Discourse: Textual analysis for social research. London: Routledge.

Fink, L. D. (2013). Creating significant learning experiences: An integrated approach to designing college courses. San Francisco: Jossey-Bass.

Gee, J. P., \& Handford, M. (2014). The Routledge Handbook of Discourse Analysis. London: Routledge.

Keller, R. (2011). Diskursforschung. Eine Einführung für SozialwissenschaftlerInnen. Wiesbaden: VS Verlag.

Kolb, D. A. (1984). Experiential Learning. Englewood Cliffs, NJ: Prentice Hall.

Killick, D. (2015). Developing the Global Student. Higher education in an era of globalisation. London \& New York: Routledge.

Leask, B. (2015). Internationalizing the Curriculum. London \& New York: Routledge.

Le Grange, L. (2016). Decolonising the university curriculum. South African Journal of Higher Education, 30(2), 1-12. http://dx.doi.org/10.20853/30-2-709

Mayring, P. (2014). Qualitative content analysis: theoretical foundation, basic procedures and software solution. Klagenfurt, 2014. http://nbnresolving.de/urn:nbn:de:0168-ssoar-395173

Sambell, K., \& McDowell, L. (1998; published online 2006). The construction of the hidden curriculum: messages and meanings in the assessment of student learning. Assessment \& Evaluation in Higher Education, 23(4), 391-402. http://dx.doi.org/10.1080/0260293980230406 


\section{Author}

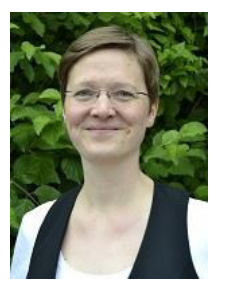

Angelika THIELSCH || Georg-August-Universität Göttingen, Section for Teaching and Learning in Higher Education \| Waldweg 26, D-37073 Göttingen

angelika.thielsch@zvw.uni-goettingen.de 
Listening out and dealing with otherness.

A postcolonial approach to higher education teaching
Arts and Humanities in Higher Education 0 (0) $1-17$

(C) The Author(s) 2019

(c) (1) (3)

Article reuse guidelines: sagepub.com/journals-permissions DOI: I0.1 I77/I4740222/9832459 journals.sagepub.com/home/ahh

@SAGE

\title{
Angelika Thielsch
}

Teaching and Learning in Higher Education, University of Göttingen, Germany

\begin{abstract}
Postcolonial pedagogy invites academic teaching staff to create situations, in which hegemonic modes of knowledge production can be critically reflected and one's own entanglement as disciplinary socialised member of (western) academia experienced. Such a postcolonial approach has been applied to a seminar in the context of cultural musicology and its impact on teaching and learning analysed. In this paper, the findings of the accompanying research are presented and discussed in relation to the concept of Bildung, theories on individual learning (in higher education) and current processes to internationalise the curricula. Throughout the argumentation, I will demonstrate how postcolonial pedagogy may cause the construction of otherness and why this simultaneously constitutes the biggest challenge as well as the profoundest reward when applying such an approach to university teaching. In addition to that, this paper introduces a definition of postcolonial pedagogy and offers recommendations to foster its implementation in higher education contexts.
\end{abstract}

\section{Keywords}

Postcolonial pedagogy, modes of knowledge production, hidden curriculum, otherness

\section{Corresponding author:}

Angelika Thielsch, Teaching and Learning in Higher Education, University of Göttingen, Waldweg 26, 37073 Göttingen, Germany.

Email: angelika.thielsch@zvw.uni-goettingen.de 


\section{Introducing the (methodological) setting}

'We need to be open-minded.'

A postcolonial approach to teaching has been chosen when facilitating the seminar 'Postcolonial Musicology: Hindustani Classical Music' at the University of Göttingen (Germany). In the context of this master's seminar, postcolonial theory was not merely part of the seminar content. The mode of teaching and learning was highly influenced by postcolonial ideas as well. By 'listening with postcolonial ears' ${ }^{2}$ (Solomon, 2012) a small group of students was introduced to a musical genre new to them, without learning concepts, terminology or norms used by (western) academia in advance. The seminar itself was conducted by an interdisciplinary teaching team; both trained in postcolonial theories, one - in addition to that - researching the broader seminar topic. ${ }^{3}$ It is this case example, the experiences with a postcolonial teaching approach, and the students' reaction that will be introduced and reflected in this paper. My aim is to critically discuss the benefits and challenges that derive from using such an approach in higher education and why and how this can promote the decolonisation of university teaching and learning.

The findings of this article are based on various data I collected throughout and in the aftermath of this seminar on postcolonial musicology. Being an educational developer, academically socialised in cultural anthropology and the disciplinary non-expert of the teaching team, I chose to include a scholarly perspective in our teaching and applied the roles of a participant observer and field researcher in addition to the role of an academic teacher. In this field research, I triangulated and interpreted three kinds of material: First, structured fieldnotes ${ }^{4}$ taken after each of the 14 teaching sessions. Second, fieldnotes of each planning meeting in which my colleague and I critically evaluated the challenges of postcolonial pedagogy, the reaction and needs of our students, and the steps necessary to (still) reach the intended learning outcomes together with the group. Third, reflective statements of the group of students as well as the leading teacher, which I collected via an (anonymous) online survey long after the official closing of the course, after all assignments had been performed, all grades given and received and new academic teaching and learning experiences made. I chose to offer both - students and teaching team partner - some time to process their experiences with the postcolonial approach of the seminar. Analytically combined, all three kinds of data offered the insights presented in this paper.

The motivation for choosing a postcolonial teaching approach in the first place as well as including a scholarly perspective therein was twofold: On the one hand, my team teaching partner and I sought a way to connect our postcolonial focus in research with our learner-centred teaching modes. On the other, we wanted to gain more knowledge on how to practically facilitate the decolonisation of the university, which is, especially since the start of the project on internationalisation of the curricula in 2015 (Casper-Hehne and Reiffenrath, 2017), one strategical focus at 
the University of Göttingen. While the concept of internationalisation of the curriculum lays the ground for and therefore aims at decolonising university teaching, a postcolonial approach to teaching is one way to realise this inside and beyond higher education classrooms.

Let me shortly introduce what I mean when I say postcolonial approach in relation to the seminar of this case study. As a teaching team, we decided to use a working definition of postcolonial higher education teaching, which characterised it as an approach to new information by...

- seeking to discover meanings and patterns from within the learning material,

- avoiding comparisons between the newly detected and the already known,

- using descriptions and names offered by context insiders instead of western scholars,

- critically reflecting this information in contrast to one's prior knowledge and regarding hitherto known concepts or theories in order to enhance a critically oriented process to make meaning.

Based on these guiding characteristics, the seminar on 'Postcolonial Musicology: Hindustani Classical Music' was designed.

Before presenting the seminars' realisation in detail, I will critically discuss the relevance of postcolonial approaches in academia and elaborate on its connection to the concept of Bildung as well as to basic findings on individual learning. After this, the context of the case study will be outlined against the background of the different dimension of curriculum in higher education in general and the concept of internationalisation of curricula in specific. These theoretical aspects offer the framework for this paper, whereas the two subsequent parts constitute its centrepiece. Here, I will present the results of my analysis and summarise the challenges and didactic coping strategies, while critically reflecting the experiences with this postcolonial approach to teaching. Part of this is a section in which I discuss how the experienced challenges mainly derived because of the otherness of the teaching and learning situation. Facing this otherness in higher education, is, as I will argue, the core challenge that needs to be addressed when applying a postcolonial approach to university teaching. At the end of this paper the reader will find recommendations on what to keep in mind when seeking to transfer such an approach to one's own teaching contexts. Finally, I will offer a definition of postcolonial teaching in higher education as pedagogical approach and sum up by arguing why such an approach is indeed valuable to (further) decolonise universities.

\section{Thinking about academia, the concept of Bildung and individual learning}

Academia seeks to increase the knowledge of the world by scientific research and to disseminate it via teaching that is devoted to the concept of Bildung. Referred to 
as one of the most complex and contested concepts of the German (pedagogical) tradition (Faulstich, 2002: 16), the ideas behind Bildung can be linked to the era of Enlightenment. While Enlightenment aimed at educating all mankind, at enabling them to question tradition and to (re)position themselves as subjects, Bildung can be understood as the pedagogical instrument to achieve this. Nowadays, Bildung and its feature to circle around discourses of the self $f^{5}$ is often defined as the ongoing process of dealing with the experiences individuals gather throughout their life; a process which makes a person change perceptions and understandings of others, of the world, and of her- or himself within it (Koller, 2012: 9).

Influenced by colonialism and its effect to systematically producing otherness (Taylor, 2016), the concept of Bildung has become a tool in western education to define which knowledge was worth distributing. Just like the concept of Bildung, academia itself is not unbiased, but lived, created and reproduced by people, who have been socialised within disciplinary modes of knowledge production, instilled with norms and values, which are being passed on through academic action. These modes of knowledge production are based on epistemologies that seek to confirm western hegemonic structures and aim at positioning former colonial societies at the centre of the world, hierarchically above others (see Said, 2003 [1978]; Spivak, 1994). Regarding this, Mbembe (2016: 33) points out that ' $\mathrm{t}$ ] his hegemonic notion of knowledge production has generated discursive scientific practices and has set up interpretative frames that make it difficult to think outside these frames.' Thus, concerning academia, postcolonial pedagogy can focus on revealing the power relations that regulate discourses in which academic teaching and learning (and research) take place. It can help to uncover what lies underneath and thereby enable the people involved to comprehend their role in (re)producing or changing these discourses. Academia needs to become aware of its colonial heritage in order to (re)position itself beyond and to fulfil its purpose as institutions of Bildung aware of former and historically created blind spots. This might reinforce and enhance the core notion of Bildung as concept: the holistic development of an individual, learning open-mindedly to locate her- or himself as a member of the world, while being able to uncover, understand and critically deal with the power relations existing in it. ${ }^{6}$

Whereas Bildung addresses a person's development at a more holistic level, theories of learning focus on individual processes of gathering information and making meaning. Learning theories and, especially, constructivism emphasise that each new information needs to be filled with meaning individually (Blumberg, 2009). During these mean-making processes a person critically evaluates his or her prior knowledge, decides whether the new information can be added to existing structures or whether (parts of) information already known need to be transformed (see Ambrose et al., 2010). In other words, during the process of learning a person interacts with new information and thus experiences it. In the context of postcolonial pedagogy, the importance of experience for such processes becomes even more significant. A postcolonial teaching approach focusses on individual perceptions, invites contradictory ways of understanding and seeks to avoid situations in 
which a teacher acts from an expert point of view. It therefore meets the demand to foster active learning, which is crucial for adult learning in general and higher education learning in particular (Fink, 2013).

Often academic teaching staff intuitively focusses on aspects that facilitate learning: We ask students what they already know about a topic, invite them to question and critically reflect new lines of argumentation, and offer feedback to help them carry on, all of which engages students and thus triggers active learning. Postcolonial pedagogy includes actions like these, adding a broader perspective. It does not only invite students to critically reflect content but to also question the way content emerged, is being talked about and dealt with. And this is crucial. Because when we learn, we do not only learn on the content-level, but simultaneously about the ways in which learning takes place. We are becoming used to learning contexts, strategies and methods and apply them without (always) questioning them. Why? Because it is easier not to question the how in order to focus more clearly on the what in learning processes. Learning needs to be understood and analysed with respect to its social context. As Berger and Luckmann argued decades ago in 1966, learning, like all realities, is socially constructed. And as such, learning contexts influence the way individuals construct their knowledge. Keeping this in mind, postcolonial pedagogy differs from other teaching approaches. Such a postcolonial approach asks teachers to first understand the socially constructed nature of each classroom and their own knowledge systems before including various epistemologies in their teaching. It asks them to critically reflect how these epistemologies relate to each other and how they as teachers might support students to position themselves within this plurality. It asks teachers to invite and enable students to apply an inductive perspective when being confronted with new subjects, instead of developing the need to first classify them. Such thinking is inherent to the mode of teaching applied in the case study presented here.

\section{The case study and its context}

To introduce the context of the case study, the dimensions of higher education curriculum offer meaningful insight. Referring to Carroll (2015: 104), I understand curriculum as '[...] everything that shapes the student's learning experience,' not just the mere syllabus. It is according to this basic definition that the different dimensions of curriculum in academia should be looked at. Higher education curriculum can be divided into two broader areas: The explicit and the implicit. Part of the explicit are the formal curriculum (e.g. the predefined content and forms of assessment) and the informal curriculum (e.g. activities offered by university institutions to support a students' learning) (Carroll, 2015; Leask, 2015). The implicit areas of the curriculum can be divided into the hidden curriculum (the values and norms based on which members socialised in one respective learning context shape their expectations regarding suitable teaching and learning behaviour) (Leask, 2015; Thielsch, 2017) and the null curriculum (those aspects 
regarding content as well as values and norms excluded, or in other words "[...] what is not taught and learned in a university" (Le Grange, 2016: 7)).

Current approaches to the internationalisation ${ }^{7}$ of the curriculum and especially internationalisation at home ${ }^{8}$ utilise this understanding to "[...] critique and destabilise the dominant paradigms that support the status quo" (Leask, 2015: 13) of western knowledge production. Being instruments to open campuses to the world - regarding content, global perspectives and various epistemologies - the aims of both are closely linked to and overlapping with those of decolonising university. They seek to uncover hegemonic discourses and show how these are being (re)produced by higher education institutions and the people acting within them (whether knowingly or not).

Based on this, let's take a closer look at the case study: The seminar on 'Postcolonial Musicology: Hindustani Classical Music' conducted at the University of Göttingen. Classified as module M.Mus.32 'Musical Flows, Genres, and Areas' of the humanities master's degree 'Cultural Musicology' it calls for the following intended learning outcomes: Students...

- broaden their musical repertoire,

- acquire knowledge on the cultural contexts of the genre in question,

- develop the ability to critically analyse the genre from a musical and cultural perspective,

- classify the respective phenomenon regarding its historical and regional contexts.

Within these parameters, the module offers academic staff to include musical genres from all over the world as part of the formal curriculum. It thereby fulfils one major demand of current approaches to internationalisation of the curriculum, namely to broaden the syllabus by integrating global perspectives.

Despite its openness in terms of content, the module specifications regarding the form of assessment yield more restrictive interpretations. They evoke expectations of students and teachers based on previous assessments. These expectations, influenced by the hidden curriculum of this master's degree, include that writing a term paper involves demonstrating one's ability to apply scientific theories, methodologies and lines of argumentation in a way that - so far - has been rewarded with high grades. In consequence, it might be challenging for students to transfer new information regarding (genre-specific) ways of knowledge production in their writing, if these do not correspond with the epistemologies they are used to applying. For our seminar this entailed that the openness which module M.Mus.32 offers might to some extent contradict the assessment-related expectations that are lived and (re)produced by the hidden curriculum of the study programme and the people acting within.

Based on this awareness we designed the seminar 'Postcolonial Musicology: Hindustani Classical Music' along the parameters of postcolonial teaching and labelled it as such. From the announcement to the actual teaching the seminar has been introduced as using an inductive and postcolonial approach, in which students learn about a genre new to them by listening to the music first, instead of listening to or reading an experts' introduction. Students were asked to develop their 
own paths in understanding the music, its elements and structures, without being offered genre-related terminology nor comparison to or classification in any set of musicology theory. Instead, audio and video recordings were used to become familiar with the music as well as with the various contexts of its production, representation, and performance. Within the seminar, we aimed at fostering a growing complexity in our students' understanding of Hindustani classical music as genre, while continually reflecting their perceptions, their per-auditives, ${ }^{9}$ and the processes of knowledge production inherent to them from a postcolonial point of view.

We worked within a triangle of contradictory focusses: We wanted our students to create their own meaning and negotiate it constructively within the group. At the same time, we wanted them to realise, how often and how easily they apply western norms and concepts without questioning whether they fit in that particular context or what kind of colonial thinking would be (re)produced by using them. We aimed at supporting processes of individual meaning-making, while constantly allowing insecurity by not accepting the use of hegemonic argumentation. This balancing between security and insecurity turns out to be the most difficult task when designing postcolonial teaching.

\section{Critical refection on a postcolonial approach to teaching}

At the beginning of this paper, I stated that one motivation to apply a postcolonial approach to teaching was to find out how claims of postcolonial theories might be realised and lived in teaching. While both of us in the teaching team had experiences in facilitating seminars on postcolonial theories and supervising student assessments on this topic, not one of us had thus far dared to apply the underlying notion of postcolonial studies - to question processes of knowledge production - in our teaching. How would our students - used to approximating music led by categories to listen out for - react, when being asked to do so without such guiding categories? How would this influence the atmosphere in the classroom? How would we as teachers cope with the anticipated insecurity of our students... and of ourselves in this process? Obviously, we expected to face some challenges. In this section, I first summarise what we anticipated and thus included in our didactical design. Later on, I will focus on the challenges we did not expect and how we reacted to them.

\section{Dealing with the obvious: Anticipated challenges and fruitful precautions}

Since the seminar was conducted as part of a master's programme that belongs to the broad area of cultural studies, we expected our students to be used to engaging with and discussing cultural theories in general. Even though we transparently emphasised that this seminar would be using a postcolonial approach to teaching, we dedicated two sessions to the anticipated need to become familiar with a postcolonial approach to music. Fortunately, Solomon's work (2012) on the postcolonial in ethnomusicology and the concise introduction to postcolonialism by 
Young (2003) offered valuable starting points for our discussion and comprehensible arguments for using such an approach in musicology. In addition to making the mode of teaching and learning in the seminar subject of discussion, we explicitly communicated that an approach like this would be challenging for us as teachers as well. Together with the group we collected and discussed ways to regulate our work in the seminar and jointly decided on a code of conduct. This code of conduct was visualised, documented and regularly presented in the seminar as reminder. Moreover, we used a symbolical object (in this case colourful glasses with 'PoCo' printed on the lenses) to indicate if the code of conduct had been breached. During the first sessions, one of the team teaching members was responsible to use these so-called PoCo-glasses, later on students assumed this responsibility as well. Crucial here was that every action against the code of conduct (e.g. not to use normative concepts, not to apply western terminology to describe the musical aspects experienced), whether by students of teachers, would be pointed out. That we as teachers as well could and should be subject to criticism was underlined by our positioning in the room. We decided not to sit in front, but among the group, visually reminding us and them that our primary role in the seminar was not one of non-negotiable expertise but of guidance.

Decolonising our ears was the essential aim while encountering and learning about the genre of Hindustani classical music. We expected two challenges to be caused by this: Providing enough time to listen as well as to practice how to describe what one has heard. Therefore, we included weekly listening and writing assignments as preparatory task for the respectively next session. Each week, students listened to a piece of music at home, always focussing on different aspects and formulating their experiences in writing. Each week they would receive feedback on their assignments before the beginning of the next session and were thereby equipped with assured knowledge as what to share in class and how to do so. Complementary to this regular individual feedback, the group of students was emphatically invited to give feedback regarding their learning experiences in class. We valued this feedback by opening the process of teaching for discussion, adapting the pace of the seminar as well as the complexity of the weekly assignments, and openly reflecting on individual challenges deriving from this postcolonial approach to teaching. Facilitated by the latter, we gained valuable information on the insecurity that this epistemologically challenging approach to teaching involves. Even though we expected our students as well as ourselves to experience insecurity regarding the ways to express opinions and to describe musical experiences while avoiding normative knowledge, we did not expect that the study programme's hidden curriculum would have such an impact.

\section{Dealing with the hidden curriculum: Insecurity by students and academic teaching staff}

This section offers a closer look at how exactly the hidden curriculum, '[...] the various unintended, implicit and hidden messages sent to the students [...]' 
(Leask, 2015: 8) influenced the seminar by bringing students and teachers alike in situations in which fundamental aspects of their prior experiences on how to behave and what to expect in a seminar were contradicted or challenged. In case of the students this was mainly caused by the grading system within the study programme. Moreover, the expectations on how teaching and learning in this context should work influenced our perception as teaching team as well. During our weekly meeting, when preparing and analysing the class session, two observations tended to reoccur: On the one hand, there was wonder, even frustration regarding the pace of the seminar; on the other, there was a need to offer the group of students more security by accentuating their learning successes ${ }^{10}$ more clearly. Both might have worked to undermine our teaching approach. Yet, since we critically reflected their quality and origins as teaching team, we generated a way to cope with our individual affective reactions and designed strategies to support our students while pursuing the postcolonial approach. I will present these strategies and the challenges they addressed in the following.

The insecurity of the group of students was caused by their habitual need to give 'correct' answers and to participate by offering 'the right' contributions. Although having agreed on the importance of multiple perspectives and explicitly questioning modes of knowledge production, their being used to collect correct information during a semester influenced their actions in this seminar. Herein lies the most powerful aspect of the hidden curriculum we experienced as teaching team: No matter how transparent, no matter how reassuring, it is the process of assessment that effects the learning strategies of students more than the approach to teaching. Not being able to change the parameters of assessment at that time (especially the obligatory grading and the asymmetrical power relation it causes between teaching staff and students), we instead focussed on integrating ways to provide more security for the students, while reflecting this from a postcolonial perspective. Let me give some examples: Sooner than planned we introduced genre specific vocabulary and invited the group to name perceived musical aspects (e.g. rhythmic patterns, tonal structures). Additionally, we included a critical discussion on the discursive power of language, the (colonial) strategy to name something in order to exert power over it (Ashcroft, 2003: 7-8), and reflected this with respect to the students' learning experiences in our seminar. From then on, we repeatedly discussed the concepts we collected at the beginning of the semester and considered meaningful for working in a postcolonial mode, such as alterity, third space and discourse. Henceforth, we used these concepts as abstract, yet safe levels of reflection to meet and discuss the experiences in class. This way of re-grouping on theoretical ground helped our students (as much as us) to proceed on the content level. Furthermore, because describing one's listening experiences (even while knowing some vocabulary) was challenging, we offered ways of visualisation to express the perceived without using words. Different forms of visual representations in Hindustani classical music were compared, their advantages and disadvantages discussed, and thereby we underlined that deciding to choose one form of representation 
always means to knowingly exclude some musical aspects and to attribute some with more importance than others.

Whereas these strategies proved to be helpful within individual sessions, the overall anxiety to fail the examination or receive bad grades reappeared various times. One reason for this became obvious during a discussion with the group, in which one student revealed the cause of her recent insecurity: She had found herself arguing with another lecturer regarding the way to question modes of knowledge production which she experienced in our class. ${ }^{11}$ Thus, caused by the discursive power of the hidden curriculum and the expectations deriving from it, the challenges of postcolonial teaching came upon the classroom from the inside as well as from the outside. Our students found themselves confronted with a kind of higher education teaching and learning other than expected. To better understand the impact of this otherness, the next part of this chapter offers some critical remarks.

\section{Detecting otherness. The primary challenge}

In the course of one's learning biography, various aspects influence our understanding of how the process of knowledge production should work. Entering university, this understanding reaches a new level, the educational focus shifts from a content-centred one to one that equally deals with its emergence. Learning how scholarly knowledge production is organised in general and in one discipline in particular, often constitutes a threshold for our students (see Land et al., 2003). Yet, by the means of university teaching, academic systems seek to address these thresholds and offer ways to bridge them. But when teaching is characterised as element to foster processes of academic socialisation, what does this imply for studying? Rhein (2010: 41) suggests the following: To him, the act of studying implies grasping the meaning behind scientific action and acquiring the ability to act meaningfully within and according to the same meaning system. ${ }^{12}$ Students therefore are the agents of their own socialisation and identity development. They learn how to be a student, to gather scientific information, survey new insights, and critically reflect their doing. They learn to find their way among disciplinary relevant theories and approaches and even start identifying with some of them. One might say that a new layer of their identity evolves: A scholarly self.

This newly developed scholarly self is being constructed based on its affiliation to one disciplinary community. If a person finds her- or himself in (academic) situations that challenge the values of this community or its epistemological convictions, the construction of otherness may be provoked to (re)assure the accuracy of one's own perspective. Both, identity and its other, are interdependent (Rapport and Overing, 2000: 12). Instead of being opposites to each other, they actually share the same border (Müller-Funk, 2016: 42) and in fact, any '[...] "construction" of the subject itself can be seen to be inseparable from the construction of its others' (Ashcroft et al., 2000: 11). What does this imply for postcolonial approaches in higher education teaching? Students, whilst in the middle of 
developing their scholarly self, are being asked to question the essence of their disciplines' knowledge production and to acknowledge that other ways can be equally meaningful. This value-oriented notion of otherness, which emerges because of postcolonial teaching, challenges part of a students' identity. Furthermore, it challenges them on a habitual level. During one's studies, a person gets used to learning in distinct ways. Being socialised as student, ${ }^{13}$ a person knows how to behave and what to expect in class. This includes context knowledge on assessment and grading as well, which are being challenged because of an epistemologically divers, nonnormative postcolonial approach to teaching.

Even if the constructed otherness regarding one's academic values is overcome (in class), the expectations a person has as member of a specific academic discourse still may cause contradictory expectations. Perhaps, applying a postcolonial approach might be easier with first-semester students, whose socialisation in one academic field, its norms, values and expected behaviour is just beginning. Yet, whether new or experienced, the effect of becoming aware of this impulse to construct otherness offers significant learning opportunities for students and even though it is challenging it thus constitutes an important benefit of postcolonial pedagogy. It invites students and teachers alike to examine their own knowledge concepts as well as their respective origins. By experiencing something as different, as unfamiliar and strange, we find ourselves in the process of our own identity construction. As Ahmed (2000: 55) points out: 'The strange is produced as a category within knowledge, rather than coming into being in an absence of knowledge.' Hence, dealing with the otherness we experience offers us a gateway to encounter modes of our (individual) knowledge construction and invites us to challenge them. What better way to start learning. . or teaching?

\section{Recommendations for higher education teaching}

Based on these experiences, some recommendations will be introduced, focussing on what to keep in mind when implementing a postcolonial approach to teaching. Condensed, these recommendations can be labelled as listening out and dealing with otherness. I shall start with the latter.

\section{Dealing with otherness}

As discussed above, postcolonial pedagogy may trigger resistance by students (and at times by teachers as well), when it comes to questioning or even contradicting their prior understandings on knowledge production within academic settings. When encountering this constructed otherness in a seminar, three aspects turned out to be relevant.

First, and long before the seminar starts, teachers should find another academic familiar with postcolonial thinking as ally. The main purpose of this is to provide theoretical and conceptual support during the semester. After all, one's own involvement in the hidden curriculum of a discipline as well as its hegemonic 
ways of knowledge production is complex. Thus, chances are that one might fall back into former teaching habits and/or perceptions. Being able to discuss the challenges faced regularly during the seminar and to reflect on the students' expectations helps to become aware of one's expectations regarding how academic teaching and learning should work. Working as teaching team offers the most accurate support, ${ }^{14}$ since both experience the entire teaching process and every situation in class. Still, even having a colleague as ongoing critical friend throughout the semester is invaluable.

Second, evaluate possibilities to prevent grading anxiety by the students. Check whether non-graded assessment is possible and, if this is not the case, try to divide the assessment in a graded and a non-graded part (e.g. a portfolio). Illustrate in class why assessment criteria might differ from the criteria students are familiar with (maybe encountering them just now in other seminars), thereby connecting the aims of postcolonial teaching in the intended learning outcomes and thus in the grading.

Third, offer as much transparency as possible regarding the fact that the seminar is being conducted as postcolonial seminar, including a short introduction as to what is meant by it. Once the semester starts and the group meets for the first time, reason your intentions for using a postcolonial approach, help students to understand the main goals of postcolonial theories and how this will influence the way of working during the seminar. Discuss openly how this may challenge everyone, students and teacher(s) alike, and decide together on a code of conduct for realising that kind of postcolonial perspective within the seminar. Ensure the relevance of the code by making it part of every session, e.g. by appointing one student as guard of it. If working as teaching team, this role can be taken by one teacher during the first weeks, in order to establish it as vivid part of the seminar.

Together, these three aspects constitute a basis for the second part of what can be recommended in accordance to the finding: To listen out for individual learning processes.

\section{Listening out}

Whereas dealing with the otherness of postcolonial pedagogy can (to a certain extent) be designed beforehand, this approach to teaching involves dealing with sudden individual insecurities as well. This is a challenge that might be anticipated yet needs to be explored continually. Listening out to the students' learning processes, their needs as individual learners as well as group is crucial and should therefore be included as a core aspect in one's teaching in class and beyond. After all, each individual involved in such a teaching and learning context faces her and his own challenges by encountering individually constructed versions of otherness. And as teachers we should be aware of that. Therefore, asking for and valuing student feedback on their learning experience here is as important as offering examples and content-related feedback to them. A postcolonial approach to teaching asks for explicit communication and multifaceted critical reflection. Both of 
which need time, which is why applying a flexible course design with sufficient buffer time that allows you to react to the needs of your students is necessary. Whether the insecurity of a student is caused by grading anxiety or by the concern to repeat normative thinking, as academic teacher you cannot react adequately without understanding the core of insecure behaviour. So, in a way listening out also means to try and decode the hidden curriculum that affects the various expectations inside the classroom, ${ }^{15}$ lived and (re)produced by students and teachers alike. These expectations will have an impact on how easily (or not) students and teachers adapt to a postcolonial approach to teaching.

\section{Finally. . .a definition}

Postcolonial higher education pedagogy can be defined as an inductive approach to university teaching that aims at better understanding the power relations inherent in different modes of academic knowledge production; it alters the student-teacher relationship by valuing subjectivity, openly discussing contradictory perceptions (and per-auditives), and critically reflecting presumptive expert knowledge.

If the value of postcolonial theory and criticism, as Walder (2007: 195) states, 'lies in its attention to forms of cultural representation [...],' then postcolonial pedagogy most likely receives value by its attention to the various forms of knowledge production and their intertwined connotations... which is exactly how it differs from other pedagogical approaches. It demands teaching and learning contexts to be (re)designed not only on the level of argumentation or interaction, but on the level of knowledge production itself. It is not about applying a mode of teaching in a given epistemological setting but about using one's own conviction as an academic as starting point to be challenged. Hence, a postcolonial approach to teaching does not start by planning and designing one's courses but by critically reflecting one's own scholarly self.

To sum up, I would like to apply these thoughts to the characteristics of postcolonialism, which Young (2007) compiled in his definition of the term and which we used in the seminar at the beginning of our journey:

'[P]ostcolonialism seeks to intervene, to force its alternative knowledges into the power structures of the west as well as the non-west. It seeks to change the way people think, the way they behave [...]. It disturbs the order of the world. It threatens privilege and power. It refuses the superiority of western cultures.' (Young, 2007: 7)

Applied and translated to the findings of this research, Young's concise definition helps to outline the aims of a postcolonial teaching approach as follows: Postcolonial pedagogy seeks to intervene with given epistemologies, it seeks to open discourses of knowledge production in the classroom and beyond. It seeks to change the way people think about academia and its supposedly objective nature and thus invites academics and students to change their perceptions and behaviours in teaching and learning contexts. It disturbs the asymmetrical order of 
the classroom by threatening power relations based on notions of right and wrong and thereby challenges the reproduction of normative thinking. In doing so, it refuses to value one way of knowledge (production) above another.

In this paper, I have illustrated that such an approach challenges teachers and students on various levels, since it asks them to question and (re)evaluate cornerstones of their previous academic socialisation. It dares identities to reflect the scholarly parameters based on which layers of their selves have been constructed. Summing up, I wish to emphasise that such an approach - even though challenging - is rewarding on an individual level as well as in relation to the aims of internationalising the curricula and decolonising university teaching. Postcolonial pedagogy helps our students to better understand why they learn and act in distinct ways, as individuals and as students; it helps them to better understand which kinds of power relations influence teaching and learning at Universities, how they are being (re)produced and how they can be disrupted; it irritates and challenges identities by pushing them out of their comfort zones and confronting them with their socially and academically constructed other.

If our students learn how to deal with this otherness because of a teaching approach such as described above, they learn how to listen out for and value thoughts articulated outside the frames of hegemonic knowledge and may become epistemically open-minded academics.

\section{Acknowledgements}

My profound gratitude goes to Eva-Maria van Straaten, friend, team teaching partner and amazing academic, whose creativity and joy in teaching made this seminar a rewarding experience and self-reflective adventure.

\section{Declaration of conflicting interests}

The author declared no potential conflicts of interest with respect to the research, authorship, and/or publication of this article.

\section{Funding}

The author received no financial support for the research, authorship, and/or publication of this article.

\section{Notes}

1. Structured fieldnote, $2^{\text {nd }}$ teaching session, statement by a student when asked how to implement the values and claims of postcolonialism in a higher education classroom ['Wir müssen offen sein!,' my own translation].

2. Solomon (2012: 235) defines such an approach as follows: 'By "listening with postcolonial ears" I mean moving beyond the discourse analysis of talk and writing about music to listen for how power relations are sonically constructed [...]' [emphasis in the original]. 
3. Her dissertation titled 'Listening Out for Sangìt Encounters: Dynamics of Knowledge and Power in Hindustani Classical Instrumental Music' has been published in the meantime (Van Straaten, 2018).

4. Those structured fieldnotes were based on five guiding questions I used as reflective impulses after each session. The guiding questions were the following: (1) What is the first thing you remember of today's class? (2) Which reaction by the group/a student surprised you the most? (3) At which time during class did you perceive the group as especially engaged; when as lost? (4) In which situation did you (as teaching team) struggle with the postcolonial approach; and why? (5) What will you keep in mind to support your students learning (needs) in the next session(s)?

5. In her dissertation, Heslop (2001) elaborates on the link between Bildung and the self and critically discusses its relevance for National pedagogy.

6. Thinking further, such an approach to Bildung relates closely to Klafki's understanding of critical pedagogy and its implications for Bildung as being based on selfdetermination, co-determination and solidarity (Beck et al., 2015; Klafki, 2007 [1985]).

7. There are various examples of how politics and higher education institutions foster such a broader and value-oriented understanding of internationalisation, e.g. in the European context published in 2018 as manual of the HRK (the HE rectors conference; Hochschulrektorenkonferenz) or as blog posting of the Internationalisation at home expert community members Jones and Reiffenrath (2018) of the EAIE (European Association for International Education).

8. Beelen (2011) elaborates on how both concepts, Internationalisation of the Curriculum (IoC) and Internationalisation at Home (IaH) overlap. While the latter makes explicit that acquiring international and intercultural competences should be possible at one's home campus, the former can be seen as structural approach to achieve this.

9. The term 'per-auditives' indicates that one's approximation to music itself is shaped by the person's involvement in existing discourses and the power relations inherent to them. In relation to cultural musicology Van Straaten (2016: 45) describes the term as '[...] a particular mode of listening out for certain musical parameters and elements during and conventionalized as music analysis, of the carefully listening, in the process maybe even constructed as knowledgeable and hence authoritative, cultural musicologist.'

10. Making explicit that there were learning successes on two levels: content-wise regarding the musical genre, procedure-wise regarding their acting in an unfamiliar mode.

11. Structured fieldnote, $11^{\text {th }}$ teaching session.

12. Studieren bedeutet, den Sinn wissenschaftlichen Tuns zu begreifen und die Befähigung zu erwerben, selbst gemäß dieser Sinnstrukturen tätig werden zu können“ (Rhein, 2010: 41 [emphasis in the original]).

13. See Alder (2018: 187-188) on the various factors influencing the socialisation of students.

14. Academic teaching staff describe working as a teaching team as rewarding - yet challenging - since it brings them in situation to encounter other teaching concepts and thus creates impulses to reflect on their own (see Lusk et al., 2016; Thielsch, 2016).

15. A reflective guideline to do so has been developed in the course of a research project on the experienced hidden curriculum in higher education (Thielsch, 2017).

\section{References}

Ahmed S (2013) Strange Encounters: Embodied Others in Post-coloniality. Abingdon \& New York: Routledge. 
Alder E (2018) Becoming a student of English: Students' experiences of transition into the first year. Arts \& Humanities in Higher Education 17(2): 185-203.

Ambrose SA, Bridges MW, DiPietro M, et al. (2010) How Learning Works: Seven Researchbased Principles for Smart Teaching. San Francisco, CA: Jossey-Bass.

Ashcroft B, Griffiths G and Tiffin H (2000) Post-Colonial Studies: The Key Concepts. London: Routledge.

Ashcroft B, Griffiths G and Tiffin H (2003) The Empire Writes Back: Theory and Practice in Post-Colonial Literatures. London: Routledge.

Beck EE, Solbrekke TD, Sutphen M, et al. (2015) When mere knowledge is not enough: The potential of bildung as self-determination, co-determination and solidarity. Higher Education Research \& Development 34(3): 445-457.

Beelen J (2011) Internationalisation at home in a global perspective: A critical survey of the 3 rd Global Survey Report of IAU. International Journal of Educational Technology in Higher Education 8(2): 249-264.

Berger PL and Luckmann T (1966) The Social Construction of Reality: A Treatise in the Sociology of Knowledge. New York: Anchor Books.

Blumberg P (2009) Developing Learner-Centered Teaching: A Practical Guide for Faculty. San Francisco, CA: Jossey-Bass.

Carroll J (2015) Tools for Teaching in an Educationally Mobile World. London \& New York: Routledge.

Casper-Hehne H and Reiffenrath T (2017) Hochschulbildung in globalen und lokalen Kontexten: Das Göttinger Modell einer Internationalisierung der Curricula. Interculture Journal: Online-Zeitschrift Für Interkulturelle Studien 17(27/28): 97-116.

Faulstich P (2002) Verteidigung von "Bildung" gegen die Gebildeten unter ihren Verächtern. Literatur-und Forschungsreport Weiterbildung 49(2): 15-25.

Fink LD (2013) Creating Significant Learning Experiences: An Integrated Approach to Designing College Courses. San Francisco, CA: Jossey-Bass.

Heslop J (2001) Subjectivity, Bildung, Pedagogy: 'Coming of Age' in Modernity. PhD Thesis, University of Victoria, USA.

Hochschulrektorenkonferenz (2018) HRK-EXPERTISE-Manual: Internationalisierung der Curricula. Prozessbeschreibungen aus deutschen Hochschulen. Bonn. Available at: https:// www.hrk.de/fileadmin/redaktion/hrk-expertise/3_Manuals/HRK-Expertise-Manual_ Curricula.pdf (accessed 22 December 2018).

Klafki W (2007 [1985]) Neue Studien zur Bildungstheorie und Didaktik: Zeitgemäße Allgemeinbildung und Kritisch-konstruktive Didaktik. Weinheim: Beltz.

Koller HC (2012) Bildung Anders Denken: Einführung in Die Theorie Transformatorischer Bildungsprozesse. Stuttgart: Kohlhammer.

Land R, Meyer JHF and Smith J (eds) (2003) Threshold Concepts within the Disciplines. Rotterdam \& Taipei: Sense Publishers.

Leask B (2015) Internationalizing the Curriculum. London \& New York: Routledge.

Le Grange L (2016) Decolonising the university curriculum. South African Journal of Higher Education 30(2): 1-12.

Lusk ME, Sayman D, Zolkoski S, et al. (2016) Playing well with others: Co-teaching in higher education. The Journal of the Effective Schools Project 23: 52-61.

Mbembe AJ (2016) Decolonizing the university: New directions. Arts \& Humanities in Higher Education 15(1): 29-45.

Müller-Funk W (2016) Theorien des Fremden. Tübingen: A. Francke Verlag. 
Rapport N and Overing J (2000) Social and Cultural Anthropology: The Key Concepts. London \& New York: Routledge.

Jones E and Reiffenrath T (2018) Internationalisation at home in practice. EAIE blog posting, 2018. Available at: https://www.eaie.org/blog/internationalisation-at-home-prac tice.html (accessed 22 December 2018).

Rhein R (2010) Lehrkompetenz und wissenschaftsbezogene Reflexion. Zeitschrift Für Hochschulentwicklung (5/3): 29-56.

Said E (2003 [1978]) Orientalism. London: Penguin.

Solomon T (2012) Where is the postcolonial in ethnomusicology? In: Solomon T and Nannyonga-Tamusuza S (eds) Ethnomusicology in East Africa; Perspectives from Uganda and Beyond. Kampala: Fountain Publishers, pp. 216-251.

Spivak GC (1994) Can the subaltern speak? In: Williams P and Chrisman L (eds) Colonial Discourse and Post-Colonial Theory: A Reader. New York: Colombia University Press, pp. 66-111.

Taylor CA (2016) Is a posthumanist Bildung possible? Reclaiming the promise of Bildung for contemporary higher education. Higher Education 1-17.

Thielsch A (2016) Lehrerfahrung teilen, Lehrkompetenzen erweitern - Team Teaching als Angebot zur Lehrkompetenzentwicklung bei Promovierenden und Professor/innen. In: Hartz S and Marx S (eds) Leitkonzepte der Hochschuldidaktik: Theorie - Empirie Praxis. Bielefeld: Bertelsmann, pp. 15-24.

Thielsch A (2017) Approaching the invisible. Hidden curriculum and implicit expectations in higher education. Zeitschrift für Hochschulentwicklung 12(4): 167-187.

Van Straaten EMA (2016) It slaps and it embraces! On psytrance, immersion, and potential facets of a transductive cultural musicology. In: Abels B (ed.) Embracing Restlessness: Cultural Musicology. Hildesheim, Zürich \& New York: Georg Olms Verlag, pp. 23-51.

Van Straaten EMA (2018) Listening Out for Sangīt Encounters: Dynamics of Knowledge and Power in Hindustani Classical Instrumental Music. PhD Thesis, University of Göttingen, Germany.

Walder D (2007) Decolonizing the (distance) curriculum. Arts \& Humanities in Higher Education 6(2): 187-196.

Young RJC (2003) Postcolonialism: A Very Short Introduction. Oxford: Oxford University Press.

\section{Author biography}

Angelika Thielsch holds a Magister Artium in Social and Cultural Anthropology, Gender Studies and Roman Philology. As educational developer, she focuses on individual learning and the various influences shaping a persons' (academic) learning biography. At the Georg-August-University of Göttingen, she develops offers for teaching newcomers and team teaching settings and supports academic teaching staff regarding the internationalisation of higher education. In her research, she focuses on decolonising university teaching \& learning as well as the construction of academic identities. Regarding the latter, she currently explores the connection between learning processes and the concepts of identity and alterity. 


\title{
Feedback in Team Teaching-Konstellationen. Eine Studie zur Lehrkompetenzentwicklung durch Begegnung anderer Wissensformen
}

\begin{abstract}
Zusammenfassung
Das Lehren in Team Teaching-Konstellationen kann durch die darin generierten Feedbackinformationen zur akademischen Lehrkompetenzentwicklung beitragen. Um dies zu untersuchen, wurde eine Studie zum wahrgenommenen Feedback in der als Teaching Team realisierten Lehre durchgeführt und die erhobenen Daten hinsichtlich verschiedener Feedbackquellen ausgewertet. Vor dem Hintergrund theoretischer Erkenntnisse zur Lehrkompetenzentwicklung, der Lehre als Teaching Team sowie der Ziele und Wirkungsweisen von Feedbackprozessen werden die Ergebnisse dieser Studie in der vorliegenden Arbeit zusammengefasst und diskutiert. Die Begegnung anderer Vorstellungen über das Lehren und Lernen, so wird argumentiert, ist hierbei von Bedeutung.
\end{abstract}

\section{Schlüsselwörter}

Team Teaching, akademische Lehrkompetenzentwicklung, Feedback, Begegnungen 


\section{Verortung und Aufbau der Arbeit}

Lernen erfolgt eingebettet in soziale Prozesse. So auch jene Bereiche der individuellen (Weiter-) Bildung, die das Ziel kompetenteren Wirkens in sich tragen. Spätestens subjektund kontextbezogene Lernverständnisse haben dies und die Bedeutung des Handelns in Beziehungsgeflechten hervorgehoben und so die Relevanz dessen unterstrichen, was Lernprozesse auszulösen vermag: die Begegnung mit anderen Wissensformen und damit einhergehenden Sinnstrukturen (vgl. Faulstich, 2013; Künkler, 2011; Meyer-Drawe, 2008; Stenger, 2017). Es ist im Rahmen dieser relationalen Perspektive auf menschliches Lernen, innerhalb derer sich die hier vorgestellte Forschung verortet.

Ausgehend von der Verwobenheit individuellen Lernens in sozialen Kontexten wird die Lehre in Team Teaching-Konstellationen betrachtet, um die Bedeutung der darin ermöglichten Feedbackprozesse besser zu begreifen. Feedback, so mein Ausgangpunkt, kann als Form ebenjener Informationen verstanden werden, die ein Subjekt in Begegnungen erhält. Das konkrete Lernfeld, auf das sich Feedback im Kontext dieser Arbeit bezieht, ist die (Weiter-) Entwicklung individueller Lehrkompetenzen.

Im vorliegenden Artikel skizziere ich, inwiefern die als Teaching Team realisierte Lehrpraxis einen besonderen Wert für Hochschullehrende haben kann, da sie die Anzahl der möglichen Anlässe zur Lehrkompetenzentwicklung, insbesondere die Vielfalt möglicher Erfahrungen und damit verbundener Lernprozesse, erweitert. Diese Erweiterung, so mein Argument, entsteht durch Feedbackinformationen, die in Team Teaching-Konstellationen erzeugt werden und in der Begegnung anderer Lehrverständnisse und Perspektiven ihre Qualität erfahren. Um diese Annahmen zu überprüfen, wurden Wissenschaftler*innen der Georg-August-Universität Göttingen eingeladen, ihre Erfahrungen in der Lehre als Teaching Team zu teilen. Der hierfür entwickelte Fragebogen zur Erhebung wahrgenommenen Feedbacks als Team Teaching-Mitglied beruht auf Erkenntnissen dreier Forschungsbereiche: Den Arbeiten zu lernförderlichem Feedback, zu Gestaltung und Mehrwert von Team Teaching-Konstellationen (in akademischen Kontexten) sowie zur Entwicklung individueller Lehrkompetenzen.

Zu Beginn dieses Artikels wird dieser theoretische Rahmen nachgezeichnet, bevor im nächsten Abschnitt ein Einblick in das Forschungsdesign sowie die Erhebung geboten wird. Hieran anschließend, im vierten Bereich, werde ich mittels der gewonnenen Daten darlegen, welche Rückmeldungen im gemeinsamen Lehren erzeugt und anhand welcher Feedbackquellen wahrgenommen wurden. Mit Verweis auf die Daten expliziere ich im nächsten Schritt, welche Feedbackprozesse sich aufgrund der Erkenntnisse der Studie als Team Teaching-inhärent zusammenfassen lassen und diskutiere, inwiefern diese in der Tat als Anlass zur Lehrkompetenzentwicklung genutzt werden können. Den Abschluss dieses Artikels bildet eine Anknüpfung zur eingangs offenbarten Überzeugung: dass es die Begegnung mit anderen Wissensformen ist, die dem hier untersuchten Feedback ein Mehr an Wirkungsmacht ermöglicht. 


\section{Theoretische Grundannahmen}

Die hier vorgestellte Forschung bewegt sich in einem Feld, in dem die Entwicklung akademischer Lehrkompetenzen das übergeordnete Ziel ist, das Lehren in einem Teaching Team der Kontext und die daraus generierten Feedbackinformationen ein Anlass, dieses Ziel zu erreichen. Im Folgenden werde ich einen kurzen Einblick in den Forschungsstand jeder dieser drei Bereiche gewähren, fokussiert auf die darin vorhandenen, für diese Arbeit relevanten Erkenntnisse.

\subsection{Im Dreiklang zur Lehrkompetenzentwicklung}

Akademische Lehrkompetenzen sind facettenreich. Um sie in ihrer Komplexität beschreiben zu können, wurden in der Vergangenheit verschiedene Anforderungsprofile Hochschullehrender entwickelt und Modelle generiert, um sie abzubilden (u.a. Brendel et al., 2006; Paetz et al., 2011; Reinmann, 2011). Trautwein und Merkt (2013) zeigen beispielsweise, wie die Lehrkompetenzen einer Person über ein Drei-Ebenen-Modell dargestellt werden können. Die Autorinnen haben darin neben individuellen Überzeugungen und dem Wissen über das Lehren und Lernen auch die Meta-Kognition sowie die Besonderheiten eines fachdisziplinären Kontextes einbezogen und unterstreichen, dass in der Kombination die einzelnen Komponenten von Lehrkompetenz im Handeln wirksam werden. In Modellen wie diesem finden sich die Dimensionen, die jedem Prozess der Kompetenzentwicklung inhärent sind, bereits implizit wieder. Denn obgleich sich Kompetenz erst in der Performanz erkennen lässt, braucht ihre Entstehung sowohl auf Theorie begründetes, abstraktes Wissen zur (zielgerichteten) Einschätzung und Bewältigung einer Situation als auch den Impuls, um das im Handeln Erlebte durch Reflexion mit ebenjenem (Vor-) Wissen zu verbinden und ihm so Bedeutung zu geben (vgl. Heiner, 2012; Klinger et al., 2013; Rhein, 2010; Roxå et al., 2008; Fink, 2013). ,Kompetenzerwerb bedeutet damit nicht mehr nur bloßes Hinzufügen von Wissen oder bloße Praxis, sondern ein ständiges situationsabhängiges Reorganisieren und Integrieren von ,erfahrenen“ Wissensstrukturen" (Gruber \& Rehrl, 2005, S. 11). Auf die Relevanz der Erfahrung im Prozess der Kompetenzentwicklung möchte ich an dieser Stelle näher eingehen.

Erfahrung kann verstanden werden als eine auf Handlung basierende Information, die durch subjektive Wahrnehmung erzeugt wird und als episodische Kenntnis über die Qualität und Verortung bestehenden Wissens innerhalb eines spezifischen Kontextes Auskunft gibt. Erfahrungen existieren nicht unabhängig vorhandener Wissensbereiche einer Person, wirken als solche jedoch erst dann förderlich für die eigene Kompetenzentwicklung, wenn sie durch reflexive Praxis in Relation des jeweiligen Vorwissens ergründet werden (vgl. Echterhoff, 2013; Gruber \& Rehrl, 2005; Meyer-Drawe, 2003). In dieser Grundannahme lässt sich die Verbindung zum Prozess des Lernens allgemein ziehen, der - mitunter als Erfahrung an sich gefasst - dann als durchlaufen verstanden wird, wenn ein Individuum eine konkrete Erfahrung zu einer Information durch Reflexion mit einem abstrakt-theoretischen Wissen darüber verknüpft. Der Ausgangspunkt kann hierbei gleichermaßen in der abstrakten Kenntnis wie auch im bewussten Erfahren liegen (u.a. Faulstich \& Bracker, 2015; Kolb, 1984; Meyer-Drawe, 2008). In seiner Arbeit zur Bedeutung erfahrungsbezogener Lernprozesse hebt Fink (2013) hervor, dass eine solche Erfahrung 
zweierlei Qualitäten haben kann: Die des Selber-Tuns und die des Beobachtens (ebd., S. 116f.). Beide können in der (unerwarteten) Begegnung anderer Wissensstrukturen und -formen in Prozesse des Lernens münden.

Akademische Lehrkompetenzentwicklung kann im Anschluss an diese Überlegungen operationalisiert werden als prozesshaftes Zusammenspiel der Aneignung abstrakten Wissens über das Lehren und Lernen an Hochschulen sowie der bewussten Erfahrungen in und mit Lehrsituationen, die durch Reflexion zueinander in Beziehung gesetzt werden.

Ein solcher Prozess benötigt Anlässe, damit er auf der einen oder anderen Ebene vorhandener Wissensstrukturen wirken kann. Dass derlei Anlässe vielfältig sind, das hat die Studie von Trautwein und Merkt (2013) verdeutlicht. Obgleich die eigene Lehrpraxis damit verwoben die Rückmeldungen der Studierenden - hier am häufigsten genannt wurde, wird dem Austausch mit Kolleg*innen sowie anderweitigen Informationsquellen großer Wert beigemessen (ebd., S. 192ff.). Ist es doch im Austausch miteinander, dass wir anderen Verständnissen begegnen und mitunter von ihnen irritiert werden. Dass dies förderlich im Sinne der Lehrkompetenzentwicklung sein kann, deckt sich mit einer von Rhein (2010) formulierten Annahme. Er stellt fest, dass Lehrkompetenz mutmaßlich dann gefördert wird, ,,wenn nicht nur die Logik von (auf Wissenschaft bezogenen) Lehr-LernProzessen expliziert, sondern auch auf die Wissenschaftslogik reflektiert wird und die Knotenpunkte dieser beiden Logiken ausbuchstabiert werden“ (ebd., S. 31). Eine Möglichkeit, die in Team Teaching-Konstellationen gegeben ist.

\subsection{Team Teaching-Konstellationen in der Hochschule}

Im akademischen Kontext kann das gemeinsame Lehren auf verschiedenen Ebenen einen Mehrwert bieten, sowohl für die beteiligten Lehrenden als auch für ihre Studierenden?. Und obgleich empirische Forschungen hierzu - insbesondere im deutschsprachigen Raum - bislang überschaubar sind, so gibt es doch Einigkeit hinsichtlich folgender Aspekte, die ich im Weiteren kurz erläutern werde:

- Team Teaching kann in verschiedenen Varianten oder Modellen realisiert werden.

- Team Teaching eignet sich für den Einsatz in akademischen Kontexten.

- Team Teaching bietet den Beteiligten Anlass zur Lehrreflexion.

Unter dem Begriff Team Teaching kann weit gefasst eine Lehrsituation verstanden werden, in der zwei oder mehr Expert*innen gemeinsam substantielle Bereiche eines Themas im Rahmen einer zusammen durchgeführten Lehrveranstaltung für eine Gruppe von Studierenden vollständig realisieren - von der Planung des Syllabus und einzelner Sitzungen sowie der Prüfungsleistungen - und sich dabei in einem physischen Raum befinden (vgl. Bacharan et al., 2008, Friend et al., 2010; Lusk et al., 2016). In ihren Ausprä-

${ }^{1}$ Insbesondere im Bereich der Lehramtsausbildung wird auf Team Teaching an Hochschulen gesetzt, um die zukünftigen Lehrenden früh im Umgang mit diversen Studierendengruppen zu üben (u.a. Bacharan et al., 2008) oder ihre Kooperationsfähigkeiten für den späteren Schulbetrieb zu fördern (u.a. Drossel \& Willems, 2014; Lusk et al., 2016). 
gungen wird basierend auf dieser allgemeinen Definition zwischen verschiedenen Modellen $^{2}$ differenziert, die gemeinsam oftmals als Co-Teaching gefasst werden und von denen lediglich eines im engeren Sinn als Team Teaching klassifiziert wird. Ein solches Team Teaching zeichnet sich dadurch aus, dass beide Lehrenden gleichermaßen und in der Regel gleichgewichtet Anteil an der Gestaltung und Durchführung einer Lehrveranstaltung haben und - so sollte m.E. ergänzt werden - von den Studierenden als gleichberechtigte Lehrende wahrgenommen werden. Es ist diese Variante, die im vorliegenden Artikel überwiegend betrachtet wird.

Allen Varianten gemein ist, dass ein Teaching Team den Studierenden eine größere Perspektivenvielfalt auf ein Thema gewährt kann. Verschiedene Autor*innen bezeichnen dies als besonderen Mehrwert für den akademischen Lehrkontext (Friend et al., 2010; Harris \& Harvey, 2000; Money \& Coughlan, 20163). Hervorgehoben werden kann hier zum einen, dass Team Teaching-Konstellationen interdisziplinäre Zugänge über Personen (und nicht rein theoretisch) in die Lehre zu integrieren vermögen und zum anderen, dass die Diskussion unterschiedlicher Standpunkte innerhalb eines Teaching Teams den Studierenden einen Einblick in wissenschaftliche Argumentation geben kann, da sie die Aushandlung verschiedener Zugänge erlebbar macht.

Gleichsam heben Lehrende selbst die Perspektivenvielfalt in der Arbeit als Teaching Team als einen Mehrwert hervor. Die beteiligten Wissenschaftler*innen erleben andere Lehrstile und wie diese auf Studierende wirken und erhalten Anlass, das eigene Lehrhandeln im Fach zu begründen. Die hierfür benötigte Bereitschaft, andere Vorgehensweisen als die gewohnten im eigenen - hier geteilten - Lehrkontext nachzuvollziehen und wertzuschätzen, wird mitunter als herausfordernd, gleichermaßen als bereichernd dargestellt (vgl. Harris \& Harvey, 2000; Letterman \& Dugan, 2004; Lusk et al., 2016; Thielsch, 2016). Insofern brauchen erfolgreich realisierte Team Teaching-Konstellationen den intensiven Austausch und offenen Dialog zwischen den Teammitgliedern. Je nach Zusammensetzung des Teaching Teams - und insbesondere bei vorhandener (statusbedingter) Hierarchie zwischen den Mitgliedern - kann dieser Austausch als Bewertung der eigenen Lehrperformanz wahrgenommen werden (vgl. Bell, 2001; Harris \& Harvey, 2000). Da der Fokus im Team Teaching jedoch nicht das gegenseitige Beobachten ist, sondern das gemeinsame Lehren, erfolgt das mögliche Feedback in Relation zum wahrgenommenen Verhalten der Studierenden und kann so die Sorge, als (Lehr)Person bewertet zu werden, abmildern.

Die Herausforderungen und Chancen von Team Teaching-Realitäten ähneln so in Teilen denen der kollegialen Hospitation bzw. peer observation. Auch hier profitieren die

${ }^{2}$ Gängige Varianten sind das Lehren an verschiedenen Stationen, das parallele Lehren in einer aufgeteilten Gruppe sowie das (insbesondere Schulkontext genutzte) bedarfsorientierte Lehren, in dem ein Teil der Lernenden eine weiterführende, der andere Teil eine wiederholende Bearbeitung eines Themas erfährt (vgl. Friend et al., 2010; Lusk et al., 2016).

${ }^{3}$ Money und Coughlan (2016) heben in ihrer Studie hervor, dass die Vorteile des alternierenden CoTeaching durch mangelnde Abstimmung der Lehrenden untereinander verringert werden. 
beteiligten Lehrenden von der Möglichkeit, kontext-spezifisches Feedback zu erhalten, aber auch geben zu können. Bei näherer Betrachtung wird zudem deutlich, dass sich der Mehrwert dieses Feedbacks über die drei Komponenten im Prozess der Lehrkompetenzentwicklung - Wissen, Erfahrung, Reflexion - begründen lässt: Durch den Fokus auf eine spezifische Lehrsituation kann Feedback zu einer konkreten Praxiserfahrung erfolgen, der Austausch darüber erzeugt einen Einblick in die dahinterliegenden, abstrakten Wissensbereiche des Lehrens, und beides initiiert im Austausch der Beteiligten Reflexionsprozesse.

Das Besondere des Lehrens in Team Teaching-Konstellationen - mit Blick auf die Lehrkompetenzentwicklung einer Person - ist nach Harris und Harvey (2000, S. 32) in den darin ermöglichten, „einzigartigen“ Feedback-Prozessen begründet. Dem zustimmend werde ich im nächsten Abschnitt die Vielschichtigkeit dieser Feedbackprozesse umreißen und ihre Bedeutung zuvor herleiten.

\subsection{Feedback und die Relevanz seines Kontextes}

Feedback wird verstanden als Information, die durch eine übermittelnde Instanz zur Verfügung gestellt wird, die einer Person etwas über die eigene Performanz oder das eigene Wissen mitteilt und die darauf abzielt, die Diskrepanz zwischen der aktuellen und der avisierten Qualität ebenjener Performanz bzw. Wissensbasis zu verringern. Diese Information hat die Qualität, vorhandene Gedächtnisstrukturen zu bestätigen oder zu ergänzen, zu restrukturieren oder zu widerlegen (Alexander et al., 1991; Butler \& Winne, 1995; Hattie \& Timperley, 2007).

Hervorheben möchte ich an dieser Stelle die Vielfalt, über die eine Feedbackinformation bereitgestellt werden kann: Der Austausch mit einer anderen Person ist nur eine Möglichkeit. Auch die unbelebte Umwelt (z. B. Medien wie Bücher oder Filme) oder vorherige Erfahrungen in einem spezifischen Kontext sowie die Reflexion darüber können derlei Informationen bereithalten. Voraussetzung ist jeweils der Handlungsbezug. Er gibt einer Information ihren relationalen Wert. So unterstreichen Hattie und Timperley (2007, S. 81): ,Feedback [... ] is a ,consequence' of performance.“ Sich dies zu vergegenwärtigen ist insbesondere mit Blick darauf relevant, dass die Feedbackquelle internal oder external sein kann. Butler und Winne (1995) argumentieren, dass externales Feedback ergänzend zum durch das Individuum selbst ermöglichten internalen Feedback wirkt. Die Lernenden „filter information provided by external feedback through knowledge and beliefs, applying conditional knowledge to identify cues" (ebd., S. 264). Das individuelle Vorwissen einer Person, ihre Einstellungen und Ziele sind hierbei die Basis, auf der angebotenes externes Feedback verarbeitet wird. Somit ist Feedback nicht nur stets eingebettet in einen spezifischen Lernkontext, auch obliegt es der Feedback-empfangenden Person, dieses basierend auf den eigenen Wissensstrukturen, Überzeugungen und Zielen - anzunehmen, abzuwandeln oder auch gänzlich abzulehnen (Hattie \& Timperley, 2007, S. 82).

Studien zur Realisierung von Feedback in Lernkontexten zeigen, dass sowohl Feedbackquelle und -inhalt als auch der Modus des Übermittels sowie der Feedback-Anlass einen Einfluss darauf haben, wie eine Information aufgenommen wird. Weiter wird angenommen, dass die Qualität einer Feedbackinformation als höher eingeschätzt wird, je elaborierter sie ist (vgl. für einen Überblick Brinko, 1993; Hattie \& Timperley, 2007; Hattie 
\& Yates, 2014). Neuere Untersuchungen beziehen zudem neben aufgaben-, prozess- und selbst-(regulations)bezogenen Ebenen von Feedback auch die des peer Austauschs untereinander - folglich diskursive Komponenten - mit ein (vgl. Dreiling et al., 2018; Lotz, 2016).

Unabhängig von der jeweiligen Ebene wird eine Feedbackinformation dann als wirkungsvoll beschrieben, wenn sie Fragen zum eigenen Lernfortschritt in Relation eines konkreten Zielkontextes zu beantworten vermag: Was genau ist mein Ziel (feed up), wie komme ich soweit voran (feed back) und welche Schritte sollte ich als nächstes gehen (feed forward4; Hattie \& Timperley, 2007, S. 86f.). Auch hier wird die Kontextrelevanz für die Wahrnehmung hilfreichen Feedbacks deutlich.

Übertragen auf den Kontext einer als Teaching Team realisierten Lehrveranstaltung liegt die Vermutung nahe, dass durch das gemeinsame Lehren eine Vielzahl von Feedbackinformationen ermöglicht wird. Zielperspektive eines solchen Feedbacks wäre die wahrgenommene Qualität einer Lehrhandlung, die jeweilige Information darüber ein möglicher Anlass, die eigenen Lehrkompetenzen weiterzuentwickeln. Derlei Informationen können in Team Teaching-Kontexten mindestens über die drei folgenden Feedbackquellen bereitgestellt werden:

- Feedback durch die Team Teaching-Mitglieder in der Praxis des gemeinsamen Lehrens und den damit einhergehenden Austausch.

- Feedback durch das Selbst anhand reflexiver Erkenntnisse im Zuge des Bergründens und Explizierens eigener Lehrhandlungen sowie der Wahrnehmung anderer.

- Feedback durch die Studierenden und ihre Reaktion auf das Lehrhandeln des Teaching Teams.

Jede dieser drei Quellen kann sowohl in der Planung und Besprechung von Lehreinheiten als auch unmittelbar in der Durchführung Feedbackinformationen erzeugen. In ihrer Gesamtheit sind alle drei durch den direkten Praxisbezug eng miteinander verbunden und gehen fließend ineinander über. Dass ebensolche Erfahrungen in und mit der Lehrpraxis maßgeblich für die Weiterentwicklung der eigenen Lehrkompetenzen sind, wurde oben bereits dargelegt. Inwiefern diese mit jenen Feedbackinformationen zusammenhängen können, die in Team Teaching-Konstellation entstehen, das ist Interesse der vorliegenden Arbeit.

\section{Erkenntnisinteresse und Forschungsdesign}

In diesem Bereich wird ein Einblick in das Fundament der hier präsentierten Forschung gegeben. Neben Informationen zum Kontext und dem Erkenntnisinteresse der Erhebung

\footnotetext{
${ }^{4}$ Price und Kolleg*innen (2010, S. 279) heben hervor, dass insbesondere in akademischen Kontexten der Fokus ein feed forward avisieren sollte, weniger ein feed back. Ein Gedanke, an den die Studie von Wimshurst und Manning (2013) anknüpft.
} 
soll vor allem das Design des Fragebogens vorgestellt werden, mit dem die hier besprochenen Daten generiert wurden.

\subsection{Der Kontext und die Forschungsfrage}

Das Design der im Sommer 2018 durchgeführten Forschung ist eingebettet in den Kontext eines hochschuldidaktischen Team Teaching-Programms. Knapp 200 Personen haben zwischen Sommersemester 2010 und Wintersemester 2017/2018 an einem solchen Programm der Hochschuldidaktik der Universität Göttingen ${ }^{5}$ teilgenommen, in dem das gemeinsame Lehren komplementär zu und in Verschränkung mit einer hochschuldidaktischen Qualifizierung für den wissenschaftlichen Nachwuchs erfolgt. Die Teams setzen sich in der Regel ${ }^{6}$ durch eine lehrerfahrene und eine in der Lehre unerfahrene Person zusammen. Obgleich die Realitäten der Teaching Teams - wie nicht anders zu erwarten von Vielfalt geprägt sind, so ist durch die Einbettung in das hochschuldidaktische Programm der Rahmen für eine Vergleichbarkeit gegeben. Über ein Semester haben die Teaching Teams eine Lehrveranstaltung gemeinsam geplant und durchgeführt und waren dabei überwiegend beide während der Lehrveranstaltung im Raum. Die gängigen Varianten des Team Teaching sind neben einer abwechselnd stattfindenden Lehre (mit Wechsel innerhalb der Sitzung oder dem Wechsel mit wöchentlicher Aufteilung) auch die alleinige Lehre des lehrunerfahrenen Team Teaching-Mitglieds. Während Lehreinsteiger*innen aufgrund der Verschränkung mit einer hochschuldidaktischen Qualifizierung lediglich ein Mal in dieser Rolle an dem Programm teilnehmen können, steht die Teilnahme für Lehrerfahrene wiederholt offen.

Bezugnehmend auf diesen Kontext sowie die oben vorgestellten theoretischen Grundlagen wurde der hier verwendete Fragebogen mit Blick auf das wahrgenommene Feedback in der Lehre als Teaching Team entwickelt. Feedback, so wurde oben dargestellt, erlangt seine Bedeutung durch den jeweiligen Kontext und das damit verbundene Ziel, die eigene Handlungsfähigkeit zu verbessern, in diesem Fall die Qualität der eigenen Lehrhandlungen. Hieran anschließend gehe ich von folgenden Hypothesen aus:

Hypothese 1: Die Weiterentwicklung der eigenen Lehrkompetenzen war Anliegen der Teilnehmenden des Team Teaching-Programms.

Hypothese 2: Das hierbei wahrgenommene Feedback adressiert jene Bereiche, die zur individuellen Lehrkompetenzentwicklung relevant sind.

Hypothese 3: Das wahrgenommene Feedback wird durch das gemeinsame Lehren beeinflusst.

${ }^{5}$ Die Team Teaching-Programme werden in Kooperation bzw. enger Abstimmung mit den Graduiertenschulen der Universität angeboten.

${ }^{6}$ Mitunter werden Teaching Teams aus zwei lehrunerfahrenen und einer lehrerfahrenen Person gebildet. In dieser Konstellation lehren entweder alle drei gemeinsam oder es wird gemeinsam geplant und ausgewertet, wohingegen nur die Lehreinsteiger*innen im Raum die Lehre gestalten. 
Hypothese 4: Die möglichen Feedbackinformationen werden je nach Lehrerfahrung unterschiedlich stark wahrgenommen.

Neben diesen zu überprüfenden Hypothesen besteht mein Erkenntnisinteresse darin, zu ergründen, ob die gewonnen Daten die Interpretation zulassen, dass die wahrgenommenen Feedbackinformationen ihren Wert durch die Begegnung anderer Perspektiven und Wissenslogiken erfahren. Die übergeordnete Forschungsfrage des Vorhabens lautet, die vier Hypothesen sowie das weitere Erkenntnisinteresse vereinend, wie folgt: Inwiefern bieten die in Team Teaching-Konstellationen erzeugten Feedbackinformationen Lehrenden unterschiedlicher Lehrerfahrungen (grundsätzlich) Anlass zur akademischen Lehrkompetenzentwicklung?

Bevor die Entwicklung des hier verwendeten Instruments erläutert wird, sollen zunächst die Rahmenbedingungen der Studie vorgestellt werden.

\subsection{Die Erhebung}

Im Rahmen der Erhebung wurden 184 Personen erfolgreich? per Email eingeladen, einen Online-Fragebogen auszufüllen, darunter 68 Lehrerfahrene und 116 Lehreinsteiger*innen. Die Rücklaufquote zur Teilnahme nach vier Wochen (bei einmaliger Erinnerung) lag bei $38 \%\left(n=70^{8}\right.$, davon 24 Lehrerfahrene, $35 \%$, und 46 Lehrunerfahrene, $\left.40 \%\right)$. Die Erhebung bestand neben einer einleitenden Information sowie einem abschließenden Dank aus vier Abschnitten zweier Inhaltsbereiche. Zunächst wurde anhand weniger Items Angaben zur Person erfragt, um die später ermittelten Einschätzungen kontextualisieren zu können. Darüber hinaus wurde erhoben, mit welcher Erwartung hinsichtlich der Lehrkompetenzentwicklung man am Programm teilnahm (oder erneut teilnimmt). Anschließend wurde die Einschätzung zu Aussagen folgender Bereiche erbeten: Gestaltung des Team Teaching allgemein, Erfahrungen während der Seminardurchführung, Erfahrungen während der Planung, Wahrnehmungen der Team Teaching-Mitglieder sowie Einschätzung zur Grundlage der eigenen Lehrkompetenzen. Die eigene Einschätzung erfolgte auf einer Vierer-Skala mit Möglichkeit, sich zu enthalten.

Für beide Gruppen wurde mit dem gleichen Instrument gearbeitet. Dennoch wurden zwei Fragebögen erstellt, um die Besonderheit der Rollen innerhalb der hier untersuchten Team Teaching-Zusammensetzung erfassen zu können. Der Fragebogen an die lehrerfahrenen Personen wies vier zusätzliche Items auf, die alle im ersten inhaltlichen Bereich angesiedelt waren: eines zur Anzahl der Teilnahmen und drei im Bereich der eigenen Ziele. Letztere Items wurden eingefügt, um die gruppenspezifische Motivation der Erfahrenen zur Teilnahme am Programm zu erfassen. In Ergänzung zur intendierten Kompetenzentwicklung kann für diese Gruppe davon ausgegangen werden, dass die Unterstützung des wissenschaftlichen Nachwuchses für sie ein zentrales Motiv war.

\footnotetext{
7 Erfolgreich bedeutet, dass die Email ohne Fehlermeldung versendet werden konnte.

${ }^{8} 50 \%$ davon gaben an, weiblich zu sein, 43 \% männlich, die Übrigen machten keine Angabe. 
Tabelle 1 bietet eine erste Orientierung zur inhaltlichen Ausgestaltung sowie der jeweiligen Anzahl der verwendeten Items. Die Items, die für die nachstehende Analyse genutzt wurden, stammen größtenteils aus Inhaltsbereich B, in dem Aussagen zum wahrgenommenen Feedback individuell bewertet werden sollten. Jedoch wird anhand einzelner Items aus den anderen Bereichen herausgearbeitet, inwiefern die Angaben sich unterscheiden, wenn sie von einer lehrerfahrenen oder einer lehrunerfahrenen Person stammen oder wenn eine bestimmte Ausprägung der Lehre als Teaching Team gewählt wurde.

\begin{tabular}{|l|l|l|l|}
\hline \multicolumn{2}{|l|}{ Inhaltlicher Bereich } & Item-Beschreibung & $\begin{array}{l}\text { Anzahl } \\
\text { Einsteiger*in | Erfahrene* }\end{array}$ \\
\hline A1) & $\begin{array}{l}\text { Einordnung des Kontextes und der } \\
\text { Distanz zur Wahrnehmung }\end{array}$ & $\begin{array}{l}\text { Angaben zur Person, zum disziplinären und institutio- } \\
\text { nellen Kontext sowie der Teilnahme an einem Team } \\
\text { Teaching-Programm }\end{array}$ & $4 \mid 5$ \\
\hline A2) & $\begin{array}{l}\text { Feedback-Ziel Lehrkompetenzent- } \\
\text { wicklung }\end{array}$ & $\begin{array}{l}\text { Bewertung von Aussagen mit dem Impuls: } \\
\text { Ich habe am Team Teaching-Programm teilgenommen... }\end{array}$ & $\begin{array}{l}4 \mid 7 \\
\text { Sonstiges }\end{array}$ \\
\hline A3) & $\begin{array}{l}\text { Realisierte Varianten und inhären- } \\
\text { ten Erfolgsfaktoren von Team } \\
\text { Teaching-Konstellationen }\end{array}$ & $\begin{array}{l}\text { Bewertung von Aussagen mit dem Impuls: } \\
\text { Das Team Teaching-Seminar... }\end{array}$ & $8 \mid 9$ \\
\hline B) & $\begin{array}{l}\text { Im Team Teaching wahrgenom- } \\
\text { menes Feedback in Relation zu } \\
\text { Bereichen der Lehrkompetenz- } \\
\text { entwicklung }\end{array}$ & $\begin{array}{l}\text { Bewertung von Aussagen mit den Impulsen: } \\
\text { Während des gemeinsamen Lehrens... | Bei der Vor- und } \\
\text { Nachbereitung des gemeinsamen Lehrens... | Mein*e } \\
\text { Team Teaching-Partner*in... | Meine Lehrkompetenz... }\end{array}$ & $\begin{array}{l}39 \\
\text { Sonstiges }\end{array}$ \\
\hline
\end{tabular}

Tab. 1: Inhaltliche Bereiche der Erhebung und Beschreibung der Items

\subsection{Der Fragebogen: Entstehung und kritische Reflexion}

Eine Herausforderung im Design dieser Studie war es, dass für die Erhebung der avisierten Lehrkompetenzentwicklung nicht auf Items eines bestehenden Instruments zurückgegriffen werden konnte, sondern es selbst eines zu entwickeln galt. Dazu wurde das Konstrukt der Lehrkompetenzentwicklung basierend auf den oben dargelegten theoretischen Annahmen expliziert, die in 2.1 skizzierte Operationalisierung erweitert und Aussagen zur Erfassung des Konstrukts formuliert. Folgende Konkretisierung ist hierbei entstanden:

- Wissen über akademisches Lehren und Lernen

Aussagen über die Bearbeitung theoretisch-abstrakten Wissens über das Lehren und Lernen an Hochschulen in Form eines Abgleichs, einer Bestätigung oder Ergänzung. Dieses Wissen umfasst relevante Aspekte des Lehrens und Lernens in Hochschulen, vom Übergeordneten (Ziele der Hochschullehre) zum Grundsätzlichen (Kenntnisse von Theorien und/oder Wegen zur Lernförderung) bis hin zum Fachspezifischen. Der Fokus liegt darauf, bewusst auf (neues) Wissen zugreifen zu können. Beispiel: „Während des gemeinsamen Lehrens ist für mich deutlich geworden, worin für Studierende in meinem Fach Lernhürden liegen können. “

- Erfahrungen im Lehrkontext

Erfahrungsbezogene, mitunter situativ-begründete Aussagen über das eigene Lehren. Diese Aussagen können erlebte Herausforderungen und (Lern)Erfolge betreffen, wahrgenommene Reaktionen der Studierenden auf verschiedene LehrLernhandlungen sowie fachspezifisches Erfahrungswissen. Der Fokus liegt auf der 
Beschreibung erlebter Lehrhandlungen, die für die Person von Bedeutung sind oder waren. Beispiel: „,Bei der Vor- und Nachbereitung des gemeinsamen Lehrens hat die Rückmeldung der Studierenden uns in der Wahl unserer Methoden beeinflusst."

- (Selbst)Reflexion als Lehrperson

Aussagen über wahrgenommene Impulse zur Reflexion. Diese Aussagen können das kritische Hinterfragen getätigter oder zukünftiger Handlungen sowie eigener Erwartungen betreffen oder die Begegnung anderer Wahrnehmungen beschreiben. Im Fokus stehen hier wahrgenommene Impulse, die zum Innehalten und überdenken einer Lehrhandlung geführt haben. Beispiel: „Mein*e Team TeachingPartner*in hat mein geplantes Lehrhandeln kritisch hinterfragt, so dass ich es überdenken konnte.“

Bei der Entwicklung der konkreten Fragebogen-Items wurde anschließend darauf geachtet, die oben genannten Team Teaching-inhärenten Feedbackquellen zu adressieren. So entstanden Items, die auf die drei Komponenten des Konstrukts Lehrkompetenzentwicklung rekurrierten und dabei als Feedbackquellen den Austausch im Teaching Team, die Reaktion der Studierenden sowie reflexive Erkenntnisse des Selbst mit einbezogen. Diese ergänzend wurden zusätzlich sechs Items gebildet, die die Besonderheit der Lehre als Teaching Team überprüfen sollten. ${ }^{9} \mathrm{Im}$ Vorfeld der Erhebung wurde der Fragebogen mit allen Items von drei Lehrenden mit kontextuell und disziplinär unterschiedlichen Team Teaching-Erfahrungen auf Verständlichkeit hin überprüft und uneindeutige Formulierungen optimiert.

Das im Zuge der hier durchgeführten Erhebung entstandene Set an Items, um den Prozess der Lehrkompetenzentwicklung zu erfassen, wurde als neues Instrument erstmals ${ }^{10}$ getestet und basierend der erhobenen Daten hinsichtlich seiner Validität und Zuverlässigkeit überprüft. Hierbei konnte die oben vorgestellte Operationalisierung mittels Faktorenanalyse als ein 3-Faktoren-Modell bestätigt werden. Zwischen acht und 10 Items laden auf jeden der drei Faktoren (mit Werten zwischen .44 und .79) und es wurden insgesamt acht Variablen aufgrund zu geringer oder uneindeutiger Ladungen aus der Reihe Konstrukt-relevanter Elemente sowie für die zukünftige Verwendung des Instruments ausgeschlossen. Schließlich wurden die Reliabilitäten der drei ermittelten Skalen (Wissen, Erfahrung, Reflexion) mithilfe Cronbachs Alpha berechnet. Aufgrund ihrer Werte zwischen .78 und .89 sind sie als akzeptabel bis gut einzuschätzen. Tabelle 2 fasst dies zusammen und gibt zudem Einblick in die Mittelwerte und jeweiligen Standartabweichungen der drei Konstrukt-bezogenen Skalen.

9 Jeweils zwei Items sind entstanden, um wahrgenommenes Feedback in der gemeinsamen, Durchführung und Nachbereitung zu erfassen.

${ }^{10}$ Eine umfassende Vorstellung des Instruments und seiner Weiterführung sind nach Einsatz bei einer größeren Stichprobe geplant. Anhand der vorliegenden Studie mit Fokus auf Feedback in Team Teaching-Konstellationen wurde das Instrument zunächst pilotiert. 


\begin{tabular}{|l|c|c|c|c|}
\hline Skala & Anzahl Items & Cronbachs $\boldsymbol{\alpha}$ & Mittelwert & SD \\
\hline Wissen über akademisches Lehren und Lernen & 8 & .80 & 3,08 & 0,89 \\
\hline Erfahrungen im Lehrkontext & 10 & .78 & 3,18 & 0,91 \\
\hline (Selbst)Reflexion als Lehrperson & 8 & .89 & 2,68 & 1,12 \\
\hline
\end{tabular}

Tab. 2: Skalen des Konstrukts Lehrkompetenzentwicklung

Hinsichtlich des hier genutzten Erhebungsdesigns sind mehrere Aspekte kritisch zu reflektieren: Dass zur Durchführung der Studie ein Instrument zur Erfassung der Lehrkompetenzentwicklung erarbeitet werden musste, war gleichsam eine Hürde als auch eine Chance. Wäre die im Rahmen der Erhebung erfolgte erste Überprüfung des Konstrukts nicht positiv verlaufen, hätte keine Aussage darüber getroffen werden können, inwiefern das hier erhobene, wahrgenommene Feedback Bedeutung für die (Weiter-) Entwicklung der avisierten Lehrkompetenzen der Stichprobe haben kann. Glücklicherweise musste darauf nicht verzichtet werden. Dennoch ist notwendig, das neue Instrument zu verfeinern, auch jenseits der Lehre in Teaching Teams einsetzbar zu machen und so insgesamt weiter zu validieren. Darüber hinaus ist zur Stichprobe kritisch zu bedenken, dass es sich um Lehrende handelt, die als Teaching Team eine hochschuldidaktische Begleitung erfahren haben (bzw. im Fall der Erfahrenen, haben können) und sich regelmäßig auch außerhalb des Teaching Teams im Austausch über das gemeinsame Lehren befanden. So ist möglich, dass die Angaben zum Grad des wahrgenommenen Feedbacks davon beeinflusst wurden und daher die Interpretation der hier gewonnenen Daten stets unter Beachtung des speziellen Kontexts der Stichprobe zu verstehen ist. Schlussendlich ist anzuführen, dass die hier präsentierte Studie auf eine erste Erhebung in diesem speziellen Kontext aufbaut. Vorbereitend zur Durchführung wurden bei derselben Zielgruppe Angaben zur Wahrnehmung der eigenen Lehre als Teaching Team sowie die erlebte Bedeutung für die eigene Lehrkompetenzentwicklung qualitativ erfasst und inhaltsanalytisch ausgewertet (vgl. Thielsch, 2016). Auf Basis dieser Vorarbeit wurden die hier verwendeten Items gestaltet und das Erkenntnisinteresse begründet, den von der Zielgruppe benannten Mehrwert der Lehre als Teaching Team auch statistisch zu überprüfen. Ein Einsatz des so entstandenen Fragebogens in einem anderen Kontext würde daher eine erneute Überprüfung der Items bedürfen.

\section{Die Ergebnisse}

Die Auswertung der verfügbaren 70 Fälle erfolgte mithilfe des offenen, SPSS-kompatiblen Programms PSPP. Von den vorhandenen Daten musste kein Fall ausgeschlossen werden, die Auswertung der Fälle beider Gruppen erfolgte zusammen. Aufgrund der Rücklaufquote von knapp $40 \%$ konnte mit mehr Daten gearbeitet werden, als subjektiv aufgrund der zeitlichen Distanz zwischen Befragung und Team Teaching-Erfahrung eines Teils der Stichprobe vermutet wurde. Dass die hier verwendeten Daten zu 19 \% von Personen stammen, die heute kein Mitglied der Universität mehr sind, sowie $37 \%$ von Lehrenden, die zwischen 2010 und 2013 am Programm teilgenommen haben, ist dementsprechend erfreulich. Beides legt den Schluss nahe, dass die Erfahrung des gemeinsamen Lehrens erinnernswert war. Nachstehende Tabelle 3 fasst zusammen, mit welchem zeitlichen Ab- 
stand auf die eigene Team Teaching-Erfahrung (in diesem Kontext) zurückgegriffen wurde.

\begin{tabular}{|l|c|c|c|c|}
\cline { 2 - 5 } \multicolumn{1}{c|}{} & \multicolumn{4}{c|}{ Beginn der (letzten) Teilnahme an einem Team Teaching-Programm } \\
\hline Gruppe & $\mathbf{2 0 1 0 - 2 0 1 1}$ & $\mathbf{2 0 1 2 - 2 0 1 3}$ & $\mathbf{2 0 1 4 - 2 0 1 5}$ & $\mathbf{2 0 1 6 - 2 0 1 7}$ \\
\hline Einsteiger*innen $(n=46)$ & $17 \%$ & $20 \%$ & $28 \%$ & $35 \%$ \\
\hline Erfahrene $(n=24)$ & $8 \%$ & $30 \%$ & $13 \%$ & $50 \%{ }^{11}$ \\
\hline Gesamt $(n=70)$ & $14 \%$ & $22 \%$ & $23 \%$ & $40 \%$ \\
\hline
\end{tabular}

Tab. 3: Rücklauf nach Gruppe und Beginn der (letzten) Teilnahme

Ein Teaching Team zeichnet sich wie in 2.2 dargestellt dadurch aus, dass die Lehre gemeinsam geplant und durchgeführt wird, wobei Intensität und Konstellationen wechseln können. Die vorliegenden Daten bestätigen dies. So bewerten knapp 83 \% $(n=69)$ der Teilnehmenden die Aussage als (eher) zutreffend (16\% bzw. $67 \%$ ), dass in der Lehrveranstaltung das gesamte Teaching Team überwiegend anwesend war und folglich im Austausch auf ein geteiltes Kontextwissen zurückgegriffen werden konnte. Die Lehrgestaltung an sich erfolgte in manchen Fällen in wöchentlich wechselnder Zuständigkeit, in anderen durch die Zuständigkeit einzelner Teile einer Sitzung ${ }^{12}$. Die Daten der Erhebung zeigen hier eine breite Streuung und belegen so die Vielfalt, in der Team TeachingKonstellationen realisiert wurden. Hervorzuheben ist, dass lediglich $13 \%$ es als zutreffend, weitere $4 \%$ als eher zutreffend bewerten, dass die gemeinsame Lehre ohne vorherige Abstimmung erfolgte $(n=69)$.

Ausgehend von diesen Informationen zur Stichprobe wird im nächsten Abschnitt argumentiert, inwiefern die in $\mathbf{3 . 1}$ formulierten Hypothesen sich anhand der gewonnenen Daten bestätigen lassen.

\subsection{Ein Blick auf die Hypothesen}

Die Teilnehmenden der hier untersuchten Team Teaching-Programme - Lehreinsteiger*innen ebenso wie Lehrerfahrene - haben den Wunsch, die eigenen Lehrkompetenzen auszubauen. Diese in Hypothese 1 formulierte Grundannahme lässt sich anhand der vorliegenden Daten bestätigen, die zeigen, dass von der gesamten Stichprobe sowohl der Zuwachs des eigenen Wissens über Lehren und Lernen erwünscht war $(M=3,61)$ als auch das Interesse, neue Erfahrungen im gemeinsamen Lehren zu machen $(M=3,27)$ sowie

${ }^{11}$ Die prozentuale Verlagerung ergibt sich u.a. daraus, dass für diese Gruppe eine wiederholte Teilnahme möglich ist. So gaben 46 \% der Erfahrenen an, dass sie mit ihrer Teilnahme in 2016-2017 ein Mal in einem Team Teaching-Programm waren, während 38 \% zwei bis drei Mal und 17 \% bereits vier und mehr Mal teilgenommen hatten.

${ }^{12} 20 \%$ aller Teilnehmenden gaben als zutreffend, $13 \%$ als eher zutreffend an, das Team Teaching durch die Verteilung einzelner Teile einer Sitzung organisiert zu haben, $23 \%$ und $17 \%$ mit wöchentlich wechselnder Zuständigkeit und 16 \% bzw. 17 \% geben an, die Verteilung jede Woche neu organisiert zu haben. 
daraus Impulse zur Reflexion des eigenen Handelns zu erhalten $(M=3,48)$. Tabelle 4 fasst die Angaben beider Gruppen zu den drei Zielebenen zusammen und weist gruppenspezifische Besonderheiten aus.

\begin{tabular}{|l|l|c|c|c|}
\cline { 3 - 4 } \multicolumn{2}{c|}{} & \multicolumn{3}{c|}{ Mittelwert (SD) je Gruppe } \\
\hline Komponente & Item & Einsteiger*in & Erfahrene*r & Gesamt \\
\hline Wissen & $\begin{array}{l}\text { Ich habe am Team Teaching-Programm teilgenommen, um neues } \\
\text { Wissen über das Lehren und Lernen an Hochschulen zu erhalten. }\end{array}$ & $3,85(, 51)$ & $3,17(, 92)$ & $3,61(, 75)$ \\
\hline Erfahrung & $\begin{array}{l}\text { Ich habe am Team Teaching-Programm teilgenommen, um durch } \\
\text { das gemeinsame Lehren eine andere Perspektive auf die Lehrin- } \\
\text { halte zu erhalten. }\end{array}$ & $3,15(, 97)$ & $3,50(, 72)$ & $3,27(, 90)$ \\
\hline Reflexion & $\begin{array}{l}\text { Ich habe am Team Teaching-Programm teilgenommen, um über } \\
\text { mein Handeln als Lehrende*r zu reflektieren ( } n=69)\end{array}$ & $3,53(, 73)$ & $3,38(, 65)$ & $3,48(, 70)$ \\
\hline
\end{tabular}

Tab. 4: Gründe zur Teilnahme in Relation zum Ziel Lehrkompetenzentwicklung (i.d.R. $n=70$ )

Es lässt sich festhalten, dass - obgleich die unterschiedlichen Lehrerfahrungen auch unterschiedliche Beweggründe hervorbringen - die Zielperspektiven der Teilnehmenden vergleichbar sind und allen Bereichen der Kompetenzentwicklung zugeordnet werden können: dem Wunsch nach neuem theoretisch-abstraktem Wissen, nach konkreten Erfahrungen aus der gemeinsamen Lehre sowie nach Impulsen zur Reflexion. Zusammengenommen bieten diese Angaben die erwartete Zielperspektive für die Wahrnehmung der hier betrachteten Feedbackinformationen ab.

Ein genauerer Blick in die Daten offenbart zudem, dass die (Weiter-) Entwicklung der individuellen Lehrkompetenzen durch die Lehre als Teaching Team theoretisch ermöglicht wurde. Folglich kann auch Hypothese 2 bestätigt werden. Die in Tabelle 2 angeführten Mittelwerte der einzelnen Komponenten des Konstrukts der Lehrkompetenzentwicklung (Wissen: $M=3,08$; Erfahrung: $M=3,18$; Reflexion: $M=2,68$ ) deuten für die Stichprobe bereits an, dass die verschiedenen Dimensionen zur Kompetenzentwicklung adressiert wurden. Dies möchte ich hier verdeutlichen und zwei Zusammenhänge anbringen, die mittels Pearson-Korrelation zwischen Items des Konstrukts und den dazugehörigen unabhängigen Variablen ermittelt wurden. Das Item „Während des gemeinsamen Lehrens hat sich mein Wissen darüber vergrößert, wie ich studentisches Lernen in einem Seminar fördern kann", das der Konstrukt-Komponente Wissen zugeordnet ist, weist in den Daten mit $p<$ .031 eine signifikante Korrelation mit der dazugehörigen unabhängigen Variablen auf, in der die Teilnahme-Motiv angegeben wurde „um neues Wissen über das Lehren und Lernen an Hochschulen zu erhalten". Ähnliches ist für Items festzustellen, die der Komponente der Reflexion zuzuordnen sind. So kann beispielsweise eine Korrelation von $p<.025 \mathrm{zwi}$ schen dem Item „Mein*e Team Teaching-Partner*in hat mein geplantes Lehrhandeln kritisch hinterfragt, so dass ich es überdenken konnte“ und dem Teilnahmeziel, „um über mein Handeln als Lehrende*r zu reflektieren“, verzeichnet werden.

Nach diesem ersten Einblick in die Ergebnisse möchte ich nun vorstellen, welche Informationen die Daten hinsichtlich Hypothese 3 und so zur Annahme, dass das wahrgenommene Feedback durch das gemeinsame Lehren beeinflusst wird, bereitstellen. Hierfür wurde ebenfalls mithilfe des Pearson Korrelationskoeffizienten überprüft, wie ausgeprägt die Zusammenhänge zwischen Angaben zu Team Teaching-spezifischem Verhalten (gemeinsame Planung, überwiegende Anwesenheit im Seminar sowie Abstimmung der Inhalte) mit den Daten zum wahrgenommenen Feedback ist. Erneut soll ein exemplarischer Einblick die gefundenen Zusammenhänge skizzieren. Insbesondere die gemeinsame 
Vor- und Nachbereitung scheint die Wahrnehmung des Feedbacks zu beeinflussen, das in Team Teaching-Konstellationen möglich ist. So ist z. B. mit $p<.019$ eine positive Korrelation zwischen dem Item zur gemeinsam die Lehre vor- und nachbereitet und der Einschätzung, „die Reaktionen der Gruppe aus vorherigen Sitzungen bedacht [zu haben], um unser Lehren weiter zu gestalten" zu erkennen. Ebenso - und noch eindeutiger - ergibt sich ein Zusammenhang zwischen Angaben zur überwiegenden Anwesenheit aller Team TeachingMitglieder mit den wahrgenommenen Feedbackinformationen. Dies zeigt sich beispielsweise in der mit $p<.001$ signifikanten Korrelationen zum Item „Mein*e Team TeachingPartner* in hat im Seminar Lehrmethoden genutzt, für die ich mich bislang nicht entschieden habe." Es wird ein statistischer Zusammenhang zwischen der als Teaching Team realisierten Lehre und der Wahrnehmung lehrbezogenen Feedbacks deutlich. Der auf einen gemeinsamen Lehrkontext verweisende Austausch im Teaching Team scheint zu beeinflussen, welche Feedbackinformationen im Lehren wie stark wahrgenommen werden und so die Lehrkompetenzentwicklung einer Person zu fördern vermögen.

Schließlich wurde die letzte der oben getätigten Annahmen überprüft. Bei näherer Betrachtung der Daten zeigt sich, dass auch Hypothese 4 nicht widerlegt werden muss: Das hier erfasste, wahrgenommene Feedback findet sich je nach Gruppe - der Lehrerfahrenen sowie der Lehreinsteiger*innen - in den Daten unterschiedlich stark ausgeprägt wieder. Insgesamt ergeben sich markante Unterschiede, die bei der Gruppe der Erfahrenen als Zeichen der Souveränität im Lehren und bei der Gruppe der Lehreinsteiger*innen als Entdecken lehrrelevanter Aspekte verstanden werden können. So verweisen die Daten beispielsweise auf einen Zusammenhang zwischen der Lehrerfahrung und der Wahrnehmung, „im Seminar Vorgehensweisen als Routinen etabliert zu haben“. Mit einem Mittelwert $M=1,92(S D=1,14)$ ist diese Aussage für die Erfahrenen weniger zutreffend als für die Lehreinsteiger*innen, deren Angaben zu diesem Item einen deutlich höheren Mittelwert $M=3,09(S D=0,91)$ aufweisen. Gleichsam eindeutig erscheint der Zusammenhang zwischen der Lehrerfahrung und der Einschätzung zur Aussage, dass einem ,im Lehren deutlich wurde, welches studentisches Lernverhalten man erwartet". In diesem Fall liegt der Mittelwert der Angaben der Lehreinsteiger*innen bei $M=3,33(S D=0,67)$ und der der Erfahrenen deutlich niedriger bei $M=2,42(S D=1,02)$.

Anschließend an diese erste Überprüfung der Hypothesen wird im Folgenden ein vertiefter Einblick in die deskriptive Analyse des wahrgenommenen Feedbacks gegeben. Um zu hinterfragen, inwiefern dieses Feedback seinen Wert durch die Begegnung anderer Perspektiven und Wissenslogiken erfährt, ist die Ergebnisdarstellung hier anhand der in 2.3 benannten, Team Teaching-inhärenten Feedbackquellen strukturiert: die Teaching Team-Mitglieder aufgrund der Situation des gemeinsamen Lehrens, die dadurch ausgelösten Selbstreflexionsimpulse sowie die erlebten Reaktionen der Studierenden auf das Lehrhandeln. Zu jeder der drei Feedbackquellen wird mittels fünf exemplarischer Items dargestellt, welche Feedbackinformationen wahrgenommen wurden. Ziel der Zusammenstellung ist es, die Perspektivenvielfalt zu verdeutlichen, über die die Teaching Teams eine Rückmeldung erfahren haben. 


\subsection{Feedbackinformationen durch die Teaching Team-Mitglieder}

Die Lehrpraxis, folglich die Planung und Durchführung einer Lehrveranstaltung, ermöglicht Lehrenden eines Teaching Teams, das eigene Handeln im Prozess begründen und die dahinterliegenden Ziele explizieren zu können. Und dies nicht nur in der Auswertung einer Situation, sondern bereits in der Darlegung antizipierter Handlungen. Auf dieser Ebene kann bereits eine erste Feedbackinformation wahrgenommen werden: Die mögliche Vergewisserung, wenn das geplante Vorgehen gegenüber einem Teaching Team-Mitglied begründet und so verständlich gemacht werden konnte. Eine Erfahrung, die $43 \%$ der Teilnehmenden für sich als zutreffend erachteten und weitere $38 \%$ als eher zutreffend. Das Wissen, das für ein solches Explizieren herangezogen wurde, hat in diesem Fall durch externales Feedback eine Bestätigung erfahren können, die in der Lehre außerhalb eines Teaching Teams nicht notwendigerweise vorhanden wäre.

Nicht nur im Austausch antizipierten Lehrhandelns, auch in der Diskussion gemeinsam erlebter Lehrsituationen können wertvolle Informationen für kompetentes Lehren enthalten sein. So können Situationen aus verschiedenen Perspektiven betrachtet werden und die Wahrnehmung eines Teaching Team-Mitglieds kann die eigene Deutung erweitern. Bestätigt findet sich diese Annahme beispielsweise darin, dass über $80 \%$ der Teilnehmenden als (eher) zutreffend angeben, im Team unterschiedliche Bedarfe bei ihren Studierenden wahrgenommen zu haben. Mit 54 \% voller Zustimmung war diese Erfahrung für die Gruppe der Lehrerfahrenen hierbei deutlicher wahrnehmbar als für die Lehreinsteiger*innen. Darüber hinaus kann im Team der erkennbare Lernfortschritt thematisiert und als Orientierung für die weitere Lehre genutzt werden. Dass dies erfolgt ist, beurteilen $80 \%$ als (eher) zutreffend, und verdeutlichen somit ein Bewusstsein darüber, den Lernfortschritt der Studierenden als Rückmeldung für die Lehrgestaltung zu nutzen.

Komplementär zu Informationen dieser Art, die über den Austausch während der Vorund Nachbereitung generiert werden, kann der Lehrstil des Team Teaching-Mitglieds durch die Erfahrung des Beobachtens eine Feedbackquelle sein. Zu erleben, wie eine andere Person Lehre gestaltet und wie sie dabei methodisch vorgeht, kann als wertvolle Information im Sinne eines feed forward aufgefasst werden. Anhand zweier Beispiele möchte ich dies durch die vorhandenen Daten belegen: Zum einen geben 58 \% der erfahrenen Lehrenden an, dass die Aussage, ihr*e Team Teaching-Partner*in habe Methoden genutzt, für die sie sich bislang nicht entschieden haben, zutrifft. Zum anderen markieren $83 \%$ der Einsteiger*innen, dass die Erfahrung des gemeinsamen Lehrens für sie verdeutlicht hat, worauf sie in Zukunft bei der Lehrgestaltung achten wollen. Beide Aussagen implizieren, dass bislang nicht bekannte oder als sinnvoll bedachte Handlungsweisen nunmehr wahrgenommen wurden.

Es zeichnet sich bereits entlang der wenigen, hier skizzierten Daten ab, inwiefern das Lehren im Team Anlass bieten kann, die erlebte Lehrpraxis zu reflektieren und sich eigener Erwartungen bewusster zu werden. Welche Feedbackinformationen dadurch für Lehrende entstehen können, ist Fokus des nächsten Abschnitts. 


\subsection{Feedbackinformationen durch generierte (Selbst)Reflexionsimpulse}

Das Zusammenspiel externalen und internalen Feedbacks kennzeichnet das Lehren als Teaching Team. Feedbackinformationen können einerseits durch Reflexionsimpulse entstehen, die im Austausch mit dem Teaching Team generiert werden, andererseits aber auch durch solche, die eine Person selbst im Verarbeiten geteilter Lehrerfahrungen entwickelt. So heben beispielsweise die Lehreinsteiger*innen in ihren Angaben hervor, dass sie sich durch die Vor- und Nachbereitung des gemeinsamen Lehrens der eigenen Erwartungen an Studierende und ihr Lernverhalten bewusster geworden sind. Während sich dies in den Daten der erfahrenen Lehrenden - erwartbar eben aufgrund ihrer Erfahrung - nicht im gleichen Maße niederschlägt, zeichnet sich jedoch die Bedeutung ab, die dem Austausch über erlebte Reaktionen der Studierenden beigemessen wurde. Hier kennzeichnen 58 \% der Erfahrenen die Aussage als für sie zutreffend, weitere $29 \%$ als eher zutreffend, dass im Teaching Team Situationen reflektiert wurden, in denen das Verhalten der Studierenden verwundert hat. Diese Einschätzung liegt über den Angaben der Einsteiger*innen, die diese Aussage mit $30 \%$ als zutreffend und $37 \%$ als eher zutreffend bewerten. Auch dies lässt sich mit dem Erfahrungsunterschied erklären. Ist doch der Start in die Lehre geprägt von Verwunderung und unerwartetes Verhalten der Studierenden eher die Regel, wohingegen der Anlass, ein solches wahrzunehmen und diskutieren zu können, im Lehralltag Erfahrener möglicherweise nicht immer Raum bekommen kann und daher vermutlich stärker im Gedächtnis bleibt.

Ob implizit, im gemeinsamen Lehren entstehend, oder durch direktes, elaboriertes Feedback, Verhaltensweisen und Einschätzungen der Team Teaching-Mitglieder können Impulse dafür generieren, das Handeln als Lehrperson, die eigene Rolle und damit einhergehende Werte zu reflektieren. Über 50 \% der Fälle finden die Aussage (eher) zutreffend, dass ihr*e Team Teaching-Partner*in sie dazu veranlasst hat, sich der Erwartungen an sich als Lehrperson klarer zu werden. Und $63 \%$ finden es gänzlich zutreffend, dass ihnen durch das gemeinsame Lehren verdeutlicht wurde, was für sie selbst in der Lehre wichtig ist. $24 \%$ geben an, dass es zutrifft, weitere $39 \%$, dass es für sie eher zutrifft, dass ihre Team Teaching-Mitglieder auch ein antizipiertes Lehrhandeln kritisch hinterfragt hätten, sodass man es bereits vor seiner Realisierung überdenken konnte. Das gemeinsame Lehren und der damit einhergehende Austausch kann den Teaching Teams offenbar Feedbackinformationen auf der Prozess-, ebenso auf der Aufgabenebene bereitzustellen.

Wie in 2.2 angeführt, ist der Austausch über gemeinsam wahrgenommene Verhaltensweisen der Studierenden sowie dessen Interpretation ein Aspekt, wieso die in Team Teaching-Situationen angebotenen Feedbackinformationen leicht(er) angenommen werden können. Auch neben den darin erzeugten Reflexionsimpulsen zeichnet die Studierendenreaktion sich in den Daten als eine Quelle wertvoller Feedbackinformationen ab, wie im Folgenden beschrieben wird.

\subsection{Feedbackinformationen durch die Reaktion der Studierenden}

Sich der Reaktion der Studierenden in Lehrsituationen bewusst zu sein und sie im weiteren Lehrgeschehen zu berücksichtigen, stellt sich in den Daten als zentraler Punkt der Vor- und Nachbereitung dar. So markierten $80 \%$ der Teilnehmenden als (eher) zutreffend, 
dass dies für sie der Fall ist. Mit $67 \%$ voller Zustimmung entscheiden die erfahrenen Lehrenden sich hier eindeutiger zu dieser Aussage als die Einsteiger*innen mit $41 \%$. Diese wiederum geben überwiegend an, dass im gemeinsamen Lehren für sie die fachspezifischen Lernhürden der Studierenden deutlich geworden sind. Eine Meinung, die die Erfahrenen zu $80 \%$ als eher nicht zutreffend beurteilen, mutmaßlich deshalb, da sie die selben Hürden bereits in vorherigen Lehrsituationen entdeckten. Größere Einigkeit spiegeln die Angaben dazu wider, dass im Team Teaching erlebt wurde, wie Studierende auf die unterschiedlichen Lehrstile reagieren. $37 \%$ Aller stimmen dem zu, weitere $36 \%$ finden dies eher zutreffend. Die Annahme, dass diese Einschätzung damit zusammenhängt, dass man zunächst sich selbst der unterschiedlichen Lehrstile bewusst geworden sein ist, unterstreicht die Bedeutung dieser Daten.

Neben der Wahrnehmung, wie eine studentische Gruppe auf das Lehren eines Teaching Team-Mitglieds reagiert, wird auch die Lehrgestaltung als Ganzes in Relation zur Rückmeldung der Studierenden bedacht. Mehrheitlich geben Einsteiger*innen und Lehrerfahrene an, dass bei der Wahl einer Methode für die nächste Sitzung das vorherige Verhalten der Studierenden berücksichtigt wurde. Unterstrichen wird dies in den Angaben eines weiteren Items: Hier sind $48 \%$ der Einsteiger*innen sich einig, dass unerwartete Reaktionen der Studierenden in der Lehre sie daran erinnert haben, in der eigenen Planung flexibel zu sein. Weitere $24 \%$ beurteilen diese Aussage als eher zutreffend. Dass die erfahrenen Lehrenden mit $13 \%$ bzw. 29 \% dies für sich nicht in gleichem Umfang als relevant einschätzen, knapp die Hälfte sogar als eher nicht zutreffend, lässt vermuten, dass die schrittweise Planung der Lehre mit zunehmender Lehrerfahrung abnimmt, die Flexibilität im Lehren steigt.

Es zeigt sich, dass über die Wahrnehmung der Studierenden zum einen Anlässe generiert werden, das eigene Wissen über die Lehre (im Fach) zu bestätigen oder anzupassen und zum anderen Situationen entstehen, in denen sich verschiedene Deutungsebenen begegnen und sich in ihrer Aushandlung um die jeweils andere Perspektive erweitern können.

\section{Diskussion und Zusammenführung}

Anhand der oben vorgestellten Ergebnisse wird deutlich, dass die Feedbackquellen, die in Relation zur Lehre als Teaching Team existieren, in besonderer Weise miteinander verwoben sind und Feedbackinformationen unterschiedlicher Qualitäten ermöglichen. Im Folgenden möchte ich die hier präsentierten Ergebnisse zum wahrgenommenen Feedback hinsichtlich ihrer Bedeutung für das Lehren im Teaching Team einerseits und die (Weiter-) Entwicklung individueller Lehrkompetenzen andererseits zusammenfassen und mit Blick auf den Wert der ihnen zugrundeliegenden Begegnungsprozesse diskutieren.

\subsection{Team Teaching ermöglicht relevantes Feedback zur Lehrkompetenzentwicklung}

Die Mitglieder des Teaching Teams ermöglichen sich direktes und indirektes Feedback, das sowohl diskursiv als auch über eine beobachtbare Handlung bereitgestellt werden 
kann und Informationen auf verschiedenen Ebenen anbietet. In Relation zu wahrgenommenen oder erwarteten Reaktionen der Studierenden können erfahrene oder antizipierte Lehrsituationen verhandelt und eigene Wissens- und Interpretationsgrundlagen hinterfragt werden. Der Austausch über die geteilte Lehrrealität kann hierbei Impulsgeber für die Reflexion individueller Lehransätze sein, die je nach Erfahrung der Lehrenden (tiefer) durchdrungen, mitunter in unbewusstes Handeln übergeleitet und durch die Begegnung mit sich neu entwickelnden Lehrroutinen (wieder) bewusster wahrgenommen werden kann. Durch den Kontext des gemeinsamen Lehrens und die Fokussierung auf eine konkrete Lehrveranstaltung können Reflexionsprozesse ausgelöst werden, die sowohl im Sinne eines feed up (hinsichtlich der eigenen Ziele und Erwartungen als Lehrperson) Informationen bereitstellen können als auch im Sinne eines feed back (mit Blick auf wahrgenommenes Verhalten und getätigte Handlungen) sowie eines feed forward (die nächsten Schritte als Lehrperson fördernd). Wenn man gemäß Hattie und Timperley (2007) davon ausgeht, dass effektives Feedback Fragen zu ebendiesen drei Zielrichtungen beantworten können muss, unterstreicht dies, inwiefern das Feedback, das im Rahmen von Team Teaching-Konstellationen ermöglicht wird, die Lehrkompetenzentwicklung einer Person zu unterstützen vermag. Ein Blick darauf, welche Bereiche der Lehrkompetenzentwicklung das hier wahrgenommene Feedback adressieren kann, verdeutlicht dies weiter. Der Austausch über Lehre erfolgt eingebettet in einen konkreten Lehrkontext, in dem die Reaktionen der Studierenden ebenso wie das Lehrhandeln der Team Teaching-Mitglieder untereinander erfahren werden kann. Ein bewusstes Erleben der Lehrpraxis ist auf mehreren Ebenen gegeben, der erste relevante Bereich somit abgedeckt. Darüber hinaus kann sowohl das gemeinsame Lehren als auch die Vor- und Nachbereitung im Team das abstrakte Wissen der Teilnehmenden bestärken, hinterfragen oder erweitern und somit den zweiten Bereich im Prozess der Kompetenzentwicklung adressieren. Dies zeigt sich u.a. in der Einschätzung der Teilnehmenden, neue Wege der Lehrkonzeption sowie neues Wissen über die Lernhürden des Faches kennengelernt zu haben. Auch die Einschätzung, geplantes Lehrhandeln im Team begründen zu können (und dies ggf. auch getan zu haben), lässt den Schluss zu, dass dazu auf - mitunter ad hoc abzuleitendes - abstraktes Wissen zurückgegriffen werden muss. Die Gelegenheit, eigene Erwartungen und Überzeugungen ebenso wie Interpretationen einer Situation zu explizieren, kann daher das Wissen aus der gemeinsamen Lehrpraxis um die Ebene des Abstrakten bereichern. Schlussendlich, und als dritte Komponente im Prozess der Kompetenzentwicklung, ist das Lehren als Teaching Team wie bereits umfassend dargelegt Anlass vielfältiger Reflexionsprozesse. Nicht nur die Erfahrungen des gemeinsamen Lehrens, auch - und insbesondere - die dahinterliegenden Konzepte und Sinnstrukturen des lehrenden Individuums können hierbei adressiert werden. Es ist die mögliche Verkopplung beider Erfahrungs-Qualitäten, der des Selber-Tuns mit der des Beobachtens, die im Austausch über ein gemeinsam Erlebtes zum Tragen kommen kann. Sie vermag, die Reflexion unterschiedlichster Aspekte des Lehrens zu fördern: Erwartungen an sich und die Studierenden, welche Vorgehensund Sichtweisen man überdenken wird, was einem wichtig ist oder man im Handeln in Zukunft beachten mag. Informationen, die den Zielrichtungen eines feed up, feed back und feed forward zugeordnet werden können und so eine hohe Wirksamkeit vermuten lassen. 


\subsection{Team Teaching ermöglicht durch Begegnung anderer Perspektiven einen Mehrwert}

Aus der Lehre generierte Anlässe zur Weiterentwicklung der eigenen Lehrkompetenzen können in vielerlei Hinsicht auch im Alleine-Lehren entstehen. Allerdings stellt die hier fehlende zusätzliche Perspektive mutmaßlich einen Qualitätsunterschied dar, der sich insbesondere im Bereich der Reflexion verdeutlicht. Die alleinige Selbstreflexion erfolgt in direkter Aushandlung der eigenen Wahrnehmungen mit vorhandenen Wissensstrukturen und somit innerhalb des Filters internaler Feedbackprozesse. Die Konfrontation mit einer anderen Perspektive, anderen Einschätzungen und Werten fehlt und so die hierdurch ermöglichten, weiteren Erkenntnisse. Besonders deutlich wird dies aufgrund der in 4.2 vorgestellten Daten zur Aussage, dass in der Vor- und Nachbereitung der Lehre unterschiedliche Bedarfe der Studierenden im Team wahrgenommen wurden. Diese Unterschiede zu sehen, im Teaching Team zu diskutieren und für die weitere Lehrplanung zu nutzen, kann einen erweiterten Blick auf die wahrgenommene Lehrrealität bereitstellen. Welchen Einfluss hat es auf die eigene Wahrnehmung, wenn andere Deutungswege verhandelt werden? Erweitert sich das Spektrum möglicher Wahrnehmungen und Interpretationen in zukünftigen Lehrsituationen? Ein Indikator dafür findet sich meiner Meinung nach in den Angaben der Lehrerfahrenen zu ebenjenem Item wahrgenommener Bedarfe. Mit $54 \%$ voller Zustimmung erscheint diese Aussage für sie bedeutsamer als für Lehreinsteiger*innen (37\%). Möglicherweise, weil diese Gruppe aufgrund ihrer Erfahrung auf Lehrroutinen zurückgreift, die seit Jahren gewachsen sind, deren Wissensstrukturen auf mehreren Ebenen ergänzt, hinterfragt oder bestätigt werden können und eine Veränderung so bewusster erfahrbar ist. Dass derlei Feedbackinformationen im Austausch mit Anderen ein breiteres Vorwissen adressiert und hinterfragt, liegt nahe. Und ebendieses Hinterfragen wäre ohne die zusätzliche Perspektive möglicherweise nicht erfolgt. Das Verhalten der Studierenden kann für derlei Prozesse der Auslöser sein. Dass sich dies im Spiegel der Wahrnehmung der Teaching Team-Mitglieder zeigt, verdeutlicht die bei den Lehrerfahrenen eindeutiger verzeichnete Verwunderung über das studentische Verhalten, wie in 4.3 skizziert. Obgleich die Gruppe der Lehreinsteiger*innen dies überwiegend als relevant erachtet, geben beinahe $90 \%$ der Erfahrenen an, im Team verwunderliches Verhalten reflektiert zu haben. Ob dies daran liegt, dass derlei Austausch aufgrund seiner Häufigkeit stärker in Erinnerung geblieben ist oder ob seiner Qualität, ist hier nicht rekonstruierbar. Dass er aber in Erinnerung geblieben ist, verdeutlicht den Mehrwert, sich über einen gemeinsam erfahrenen Lehrkontext auszutauschen und so die eigene Wahrnehmung einer Lehrsituation durch eine weitere externe Feedbackinformation zu bereichern: der Sicht des Team Teaching-Mitglieds. Die Relevanz diskursiven Feedbacks wird - insbesondere im Bereich der Lehrkompetenzentwicklung einer Person - deutlich. Hier können im Austausch die Grenzen des eigenen Wissens bedacht und gleichsam neu entdeckt werden. Während der Austausch für neue Lehrende auch neues Wissen bereithalten kann, offeriert er in diesem Kontext für Lehrerfahrene die Möglichkeit des Erinnerns verinnerlichter, heute mitunter nicht mehr bewusster Sinnstrukturen. Der Austausch über die Lehre gibt Raum für Verwunderung und so für das Lernen als Lehrperson. Denn Lernen, so unterstreicht Meyer-Drawe (2008, S. 193), beginnt mit einem Aufmerken. 


\subsection{Der Versuch einer Zusammenfassung}

Was bedeuten diese Erkenntnisse mit Blick auf die Forschungsfrage? Inwiefern bieten die in Team Teaching-Konstellationen erzeugten Feedbackinformationen Lehrenden unterschiedlicher Lehrerfahrungen (grundsätzlich) Anlass zur akademischen Lehrkompetenzentwicklung? Zunächst möchte ich hierfür die vier grundlegenden Ausprägungen benennen, die sich für Team Teaching-inhärente Feedbackinformationen aufgrund der hier vorgestellten Forschung erkennen lassen. Die Lehre in Team Teaching-Konstellationen ermöglicht Feedbackinformationen durch

1. das Explizieren eigener Sinnlogiken sowie Wahrnehmungen im Teaching Team,

2. das Erfahren unterschiedlicher Lehrstile und -ansätze sowie ihrer Wirkung auf die Studierenden,

3. das Erkennen anderer Wahrnehmungen und Deutungsmuster sowie den komplementär verlaufenden Abgleich mit eigenen Wissensstrukturen und ihren Hintergründen,

4. das Erkennen eigener Erwartungen und Ziele als Lehrperson in der Wissenschaft.

In Verbindung mit den offenkundigen Aspekten des gemeinsamen Lehrens - zum einen der Lehrpraxis an sich und den damit verfügbaren Rückmeldungen zum Lehren in einem Kontext, zum anderen dem direkt übermittelten Feedback des Team TeachingMitglieds im Sinne einer handlungsbezogenen Rückfrage oder Bewertung - sind es diese Elemente, die in der Lehre als Teaching Team einen besonderen Wert für die Lehrkompetenzentwicklung einer Person haben können. Und es ist die Begegnung mit dem Anderen, die hier den Mehrwert erzeugen kann.

Die Implikationen, die sich heraus ergeben, tragen einen klaren Appell: Ob als Lehreinsteiger*in oder erfahrene Lehrperson: Sich mit anderen Perspektiven zu reiben und die Verwunderung über unerwartete Handlungslogiken zu suchen kann dabei helfen, im Lehren (noch) kompetenter zu werden. Ein erster Schritt hierfür ist, im Austausch unter Lehrenden nicht nur auf das Was und das Wie, sondern vor allem auf das Warum zu fokussieren. Ein weiterer kann darin liegen, mögliche Alltagsverständnisse über Feedback in der Lehre aufzubrechen und die darin tradierte, eindimensionale Konnotation des BewertetWerdens zu überwinden. Ein größeres Verständnis davon, woraus Feedbackinformationen entstehen können und dass ihre Bedeutung durch den Abgleich eigener Handlungslogiken und Wissensstrukturen erfolgt, kann hierfür sinnvoll sein. Hervorheben möchte ich, dass die hier vorgestellte Studie unterstreicht, dass es für Angebote zur Lehrkompetenzentwicklung - insbesondere für Lehreinsteiger*innen - bedeutsam ist, die Verbindung zur Lehrpraxis zu erschaffen. Das Anknüpfen an Handlungsroutinen stellt zudem besonders für erfahrene Lehrende eine Möglichkeit dar, um implizites Wissen erneut erfahrbar und so für künftiges Handeln bewusst zu machen. Die Lehre als Teaching Team vermag beides zu ermöglichen, erscheint bei genauerer Betrachtung für die Zielgruppe der Erfahrenen jedoch beinahe als Instrument zur Lehrkompetenzentwicklung noch wertvoller als für die Gruppe der Lehreinsteiger*innen. 


\section{Ausblick}

Im Anschluss an die Erkenntnisse dieser Arbeit ergeben sich weitere Forschungsfragen zu allen der der eingangs behandelten Felder: Die Entwicklung akademischer Lehrkompetenzen, die Bedeutung von Feedbackprozessen sowie die Möglichkeiten der Lehre als Teaching Team.

Das neu entstandene Instrument zur Erfassung der Lehrkompetenzentwicklung einer Person gilt es auszubauen und bei größerer Stichprobe sowie in anderen Kontexten, hochschulübergreifend oder jenseits der Verzahnung mit hochschuldidaktischen Elementen einzusetzen. Welche Relevanz haben die einzelnen Komponenten des Konstrukts der Lehrkompetenzentwicklung für verschiedene Zielgruppen? Welche Erkenntnisse hinsichtlich der Gestaltung hochschuldidaktischer Maßnahmen ergeben sich daraus?

Gleichsam relevant kann eine genauere Überprüfung der Feedbackinformationen in Prozessen akademischer Kompetenzentwicklung sein. Zeichnen sich Unterschiede hinsichtlich der Wirkung eines feed forward gegenüber eines feed up oder feed back ab? Welche Implikationen hätte eine solche Erkenntnis beispielsweise für die Gestaltung von Feedbackprozessen als Instrument zur Förderung der akademischen Sozialisation?

Schließlich verleiten die hier erzeugten Daten dazu, erlebte Team TeachingErfahrungen qualitativ weiter zu beforschen. Welche Prozesselemente können ermittelt werden, die unterschiedlichen Teams den Anlass gaben, im gemeinsamen Lehren regelmäßig innezuhalten und (fachspezifisch) individuelle Handlungslogiken zu begründen? Welche Reflexionsimpulse haben die Team Teaching-Mitglieder erfahren, die das gemeinsame Lehren überdauern und auch mit zeitlichem Abstand noch wirken? Derlei Informationen über die Qualität des lehrpraxis- und situationsbezogenen Austauschs in Teaching Teams könnten genutzt werden, um einen tieferen Einblick in fachspezifische Herausforderungen akademischer Lehre zu erhalten und bestehende hochschuldidaktische Maßnahmen mit diesem Wissen zu bereichern.

\section{Abschließende Bemerkungen}

Bereits im Jahr 2009 haben Roxå und Mårtensson dazu aufgerufen, die sozialen Aspekte universitärer Lehre näher zu beleuchten. Ihre Grundannahme, dass akademische Lehre auf Basis individueller Überzeugungen konstruiert und in ihrer Umsetzung durch soziale Elemente beeinflusst wird (Roxå \& Mårtensson, 2009, S. 548), lässt sich mit den hier vorgestellten Ergebnissen unterstreichen. In meiner Arbeit habe ich den Blick auf die Chancen der Lehre als Teaching Team gerichtet und verdeutlicht, dass in der gemeinsamen Lehre sowie den hiermit verbundenen Begegnungsprozessen ein besonderer Wert liegen kann. Dieser Wert ergibt sich für das Lehrhandeln an sich, aber auch für die sich hier ergebenden Anlässe zur Lehrkompetenzentwicklung der jeweiligen Wissenschaftlerin bzw. des jeweiligen Wissenschaftlers. So wurde argumentiert, inwiefern die in Team TeachingKonstellationen generierten Feedbackinformationen im Aufeinandertreffen mit anderen Perspektiven und Logiken wahrgenommen werden und sie in ihrer Vielfalt die einzelnen Bereiche im Prozess der (Weiter-) Entwicklung individueller Lehrkompetenzen zu adressieren vermögen. 
Feedback kann aus dieser Betrachtung heraus als Instrument verstanden werden, um den Kern subjektbezogener Lernzugänge in konkreten Situationen zu ermitteln und so die Bedeutung des Miteinanders, des in Beziehung-Tretens sowie der Verwobenheit individueller Lernprozesse mit sozialen Aspekten eines Kontextes (be-) greifbar zu machen.

\section{Literatur}

Alexander, P. A., Schallert, D. L. \& Hare, V. C. (1991). Coming to terms: How researchers in learning and literacy talk about knowledge. Review of educational research, 61(3), 315343.

Bacharach, N., Heck, T. W. \& Dahlberg, K. (2008). Co-teaching in higher education. Journal of College Teaching \& Learning, 5(3), 9-16.

Bell, M. (2001). Supported reflective practice: A programme of peer observation and feedback for academic teaching development. International Journal for Academic Development, 6(1), 29-39.

Brendel, S., Eggensperger, P. \& Glathe, A. (2006). Das Kompetenzprofil von HochschullehrerInnen: Eine Analyse des Bedarfs aus Sicht von Lehrenden und Veranstaltenden. Zeitschrift für Hochschulentwicklung (ZFHE), 1(2), 55-84.

Brinko, K. T. (1993). The practice of giving feedback to improve teaching: What is effective? The Journal of Higher Education, 64(5), 574-593.

Butler, D. L. \& Winne, P. H. (1995). Feedback and self-regulated learning: A theoretical synthesis. Review of educational research, 65(3), 245-281.

Dreiling, K. \& Flierl, R. \& Willems, A. S. (2018). FeeDo - Feedbackprozesse im Deutschunterricht der Oberstufe: Erste Befunde einer Pilotierungsstudie zur standardisierten Erfassung wahrgenommenen Feedbacks. In A. Krüger, F. Radisch, A. S. Willems, T. Häcker \& M. Walm (Hrsg.), Empirische Bildungsforschung im Kontext von Schule und Lehrer*innenbildung (S. 194-205). Bad Heilbrunn: Klinkhardt.

Drossel, K. \& Willems, A. S. (2014). Zum Zusammenhang von Formen der Lehrerkooperation, des Schulleitungshandelns und des Kooperationsklimas an Ganztagsgymnasien. In K. Drossel, R. Strietholt \& W. Bos (Hrsg.), Empirische Bildungsforschung und evidenzbasierte Reformen im Bildungswesen (S. 129-154). Münster: Waxmann.

Echterhoff, W. (2013). Erfahrung. Erfahrungsbildung. In M. A. Wirtz (Hrsg.), Dorsch. Lexikon der Psychologie (16. Auflage). Bern: Hogrefe Verlag.

Faulstich, P. (2013). Menschliches Lernen. Eine kritisch-pragmatistische Lerntheorie. Bielefeld: transcript Verlag.

Faulstich, P. \& Bracker, R. (2015). Lernen - Kontext und Biografie. Empirische Zugänge. Bielefeld: transcript Verlag.

Fink, L. D. (2013). Creating significant learning experiences: An integrated approach to designing college courses. San Francisco: Jossey Bass.

Friend, M., Cook, L., Hurley-Chamberlain, D. \& Shamberger, C. (2010). Co-teaching: An illustration of the complexity of collaboration in special education. Journal of educational and psychological consultation, 20(1), 9-27.

Gruber, H. \& Rehrl, M. (2005). Praktikum statt Theorie? Eine Analyse relevanten Wissens zum Aufbau pädagogischer Handlungskompetenz. Regensburg: Universität Regensburg. 
Harris, C. \& Harvey, A. N. (2000). Team teaching in adult higher education classrooms: Toward collaborative knowledge construction. New directions for adult and continuing education, Issue $87,25-32$.

Hattie, J. \& Timperley, H. (2007). The power of feedback. Review of educational research, $77(1), 81-112$.

Hattie, J. \& Yates, G. (2014). Visible learning and the science of how we learn. London \& New York: Routledge.

Heiner, M. (2012). Referenzpunkte für die Modellierung der Kompetenzentwicklung in der Lehre - Impulse für die hochschuldidaktische Weiterbildung. In R. Egger \& M. Merkt (Hrsg.), Lernwelt Universität. Entwicklung von Lehrkompetenz in der Hochschullehre (S. 167-192). Wiesbaden: VS Verlag für Sozialwissenschaften

Klinger, M., Thielsch, A. \& Wiemer, M. (2013). Kurzformate in der Hochschuldidaktik. Ein Ansatz zur Klassifizierung. In B. Berendt, A. Fleischmann, N. Schaper, B. Szczyrba \& J. Wildt (Hrsg.), Neues Handbuch Hochschullehre (L 1.20). Berlin: DUZ Medienhaus.

Kolb, D. (1984). Experiential education: Experience as the source of learning and development. Englewood Cliffs: Prentice-Hall.

Künkler, T. (2011). Lernen in Beziehung: Zum Verhältnis von Subjektivität und Relationalität in Lernprozessen. Bielefeld: transcript Verlag.

Letterman, M. R. \& Dugan, K. B. (2004). Team teaching a cross-disciplinary honors course: Preparation and development. College teaching, 52(2), 76-79.

Lotz, M. (2016). Kognitive Aktivierung im Leseunterricht der Grundschule: Eine Videostudie zur Gestaltung und Qualität von Leseübungen im ersten Schuljahr. Wiesbaden: Springer Verlag.

Lusk, M. E., Sayman, D., Zolkoski, S., Carrero, K. \& Chui, C. L. (2016). Playing well with others: Co-teaching in higher education. The Journal of the Effective Schools Project, 23, 52-61.

Meyer-Drawe, K. (2003). Lernen als Erfahrung. Zeitschrift für Erziehungswissenschaft, 6(4), 505-514.

Meyer-Drawe, K. (2008). Diskurse des Lernens. München: Wilhelm Fink Verlag.

Money, A. \& Coughlan, J. (2016). Team-taught versus individually taught undergraduate education: a qualitative study of student experiences and preferences. Higher Education, 72(6), 797-811.

Paetz, N. V., Ceylan, F., Fiehn, J., Schworm, S. \& Harteis, C. (2011). Kompetenz in der Hochschuldidaktik. Ergebnisse einer Delphi-Studie über die Zukunft der Hochschullehre. Wiesbaden: Springer Verlag.

Price, M., Handley, K., Millar, J. \& O'Donovan, B. (2010). Feedback: All that effort, but what is the effect? Assessment \& Evaluation in Higher Education, 35(3), 277-289.

Reinmann, G. (2011). Förderung von Lehrkompetenz in der wissenschaftlichen Weiterbildung: Ausgangslage, Anforderungen und erste Ideen. In M. Weil et al. (Hrsg.), Aktionsfelder der Hochschuldidaktik. Von der Weiterbildung zum Diskurs (S. 129-150). Münster: Waxmann.

Rhein, R. (2010). Lehrkompetenz und wissenschaftsbezogene Reflexion. Zeitschrift für Hochschulentwicklung, (5)3, 29-67. 
Roxå, T. \& Mårtensson, K. (2009). Significant conversations and significant networksexploring the backstage of the teaching arena. Studies in Higher Education, 34(5), 547559.

Roxå, T., Olsson, T. \& Mårtensson, K. (2008). Appropriate use of theory in the Scholarship of Teaching and Learning as a strategy for institutional development. Arts and Humanities in Higher Education, 7(3), 276-294.

Schaper, N., Reis, O., Wildt, J., Horvath, E. \& Bender, E. (2012). Fachgutachten zur Kompetenzorientierung in Studium und Lehre. Bonn: HRK projekt nexus.

Stenger, U. (2017). Phänomen Sozialität-Singularität-Identität. In M. Brinkmann, M. F. Buch \& S. S. Rödel (Hrsg.), Pädagogik-Phänomenologie (S. 79-96). Wiesbaden: Springer VS.

Thielsch, A. (2016). Lehrerfahrung teilen, Lehrkompetenzen erweitern - Team Teaching als Angebot zur Lehrkompetenzentwicklung bei Promovierenden und Professor/innen. In S. Hartz \& S. Marx (Hrsg.), Leitkonzepte der Hochschuldidaktik: Theorie Empirie - Praxis (S. 15-24). Bielefeld: W. Bertelsmann Verlag.

Trautwein, C. \& Merkt, M. (2013). Struktur und Entwicklung von Lehrkompetenz im Spannungsfeld von Überzeugungen, Konzepten und Praxis von Lehren und Lernen. In M. Heiner \& J. Wildt (Hrsg.), Professionalisierung der Lehre. Perspektiven formeller und informeller Entwicklung von Lehrkompetenz im Kontext der Hochschulbildung (S. 179210). Bielefeld: W. Bertelsmann Verlag.

Wimshurst, K. \& Manning, M. (2013). Feed-forward assessment, exemplars and peer marking: evidence of efficacy. Assessment \& Evaluation in Higher Education, 38(4), 451465. 


\section{Dank}

Die Gedanken und Erkenntnisse dieser Arbeit wären nicht möglich gewesen ohne die engagierten Lehrenden der Universität Göttingen. Nicht nur haben sie durch ihre Teilnahme an der Erhebung die grundlegenden Daten dieser Forschung beigesteuert. Auch und in erster Linie - ist es die Begegnung mit ihren Perspektiven und Sinnstrukturen, die für meine Arbeit im Rahmen der Team Teaching-Programme und für mich als Hochschuldidaktikerin allgemein eine ungemeine Bereicherung darstellen. Dafür ein herzlicher Dank!

\section{Autorin}

Angelika Thielsch, Georg-August-Universität Göttingen, Hochschuldidaktik, Göttingen, Deutschland; angelika.thielsch@zvw.uni-goettingen.de 



\title{
Curriculum Vitae
}

Angelika Thielsch

\begin{abstract}
Akademische Abschlüsse
Dr. disc. pol.

Georg-August-Universität Göttingen, Sozialwissenschaftliche Fakultät, Institut für Erziehungswissenschaft, Bildungswissenschaften, 2019

Magister Artium

Georg-August-Universität Göttingen, Sozialwissenschaftliche Fakultät, Ethnologie (Hauptfach), Geschlechterforschung, Romanische Philologie, 2006
\end{abstract}

\section{Berufliche Laufbahn}

Seit 2011 Hochschuldidaktikerin, Georg-August-Universität Göttingen

2010-2011 Hochschuldidaktikerin, TU München

2008-2010 Hochschuldidaktikerin, Universität Kassel

2007-2008 Dez. Gleichstellungsbeauftragte, Georg-August-Universität Göttingen

\section{Portfolio akademischer Tätigkeiten}

\section{Veröffentlichungen vor der Dissertation (Auswahl)}

Thielsch, A. (2016). Lehrerfahrung teilen, Lehrkompetenzen erweitern - Team Teaching als Angebot zur Lehrkompetenzentwicklung bei Promovierenden und Professor/innen. In Hartz, S. \& S. Marx (eds.), Leitkonzepte der Hochschuldidaktik: Theorie - Empirie - Praxis, S. 15-24. Bielefeld, W. Bertelsmann Verlag.

Thielsch, A. \& Wiemer, M. (2016). Wie kommen Studierende zur Wissenschaft? In Heiner, M., Baumert, B., Dany, S., Haertel, T, Quellmelz, M. \& Terkowsky, C. (eds.), Was ist gute Lebre? Perspektiven der Hochschuldidaktik, S. 275-283. Bielefeld, W. Bertelsmann Verlag.

Thielsch A., van Treeck, T. \& Vohle, F. (2014). Video-Feedback für Promovierende - Erfahrungen eines Qualifizierungskonzepts mit dem Video als Lernraum. In Rummler, K. (ed.), Lernräume gestalten Bildungskontexte vielfältig denken. Münster, Waxmann.

Thielsch, A. (2011): Learning and Self-Direction. Ways to foster Self-Directed Learning in Higher Education. In Schröttner, B. \& Hofer, Ch. (eds.), Looking at Learning - Blicke auf das Lernen: Higher Education, Language, Place Hochschule, Sprache, Ort. Graz, Leykam.

Thielsch, A. (2009): Changing masculinities in globalising South Africa. Sindiwe Magona's novel mother to mother and its value for intercultural education. In Schröttner, B. \& Ch. Hofer (Hg.), Education - Identity - Globalization/ Bildung - Identität - Globalisierung. Graz, Leykam. 


\section{Vorträge \& Tagungsbeiträge (Auswahl)}

Team Teaching in der Wissenschaft - Theorie, Praxis, Reflexion. Vortrag im Rabmen von High Noon Didaktik zu Mittag des Zentrums für Lebrkompetenz an der Karl-Franzens-Universität Graz, 25. Oktober 2017.

Linking strategy and training: A multi-faceted approach to foster internationalisation in higher education.

Vortrag mit T. Reiffenrath im Rahmen der Konferenz Professional development of lecturers: The missing link in internationalisation, The Hague, 20. September 2017.

Emotionen und Diversität als Herausforderungen im Lehren und Lernen. Diskurswerkstatt mit Hobberg, $V$. \& S. Kesseler im Rabmen der 46. Jabrestagung der dghd an der TH Köln, 7.-10. März 2017.

Lehre bewegend gestalten: Perspektive der Hochschuldidaktik. Vortrag im Rabmen der adb-Fachtagung Bewegungsförderung von Studierenden in der Lebenswelt Hocbscbule, Hannover, 9. Dezerember 2016.

Kurzformate in der Hochschuldidaktik - Aktuelle Beispiele und ein Ansatz zur Klassifizierung. Diskurswerkstatt mit Klinger, M. \& M. Wiemer im Rabmen der DOSS - Dortmund Spring School of Staff Development, TU Dortmund, 7.-9. März 2012.

Creating Teaching Portfolios through Collaboration. Beitrag für die 36. Internationale Konferenz IUT Improving University Teaching zum Thema "The Collaborative Classroom", Bielefeld, 20.07.2011.

Zwischen Wissenschaft und Service - Identitätsfindung im Dritten Raum. Workshop im Rahmen der 40. Jabrestagung der dghd und des 1. Internationalen Symposiums ProLehre, München, 3.03.2011.

Promoting (inter)cultural awareness by the use of African literature in European higher education. Beitrag für die 10. Internationale Konferenz der Association for Language Awareness "Awareness Matters. Language, Culture, Literacy", Kassel, 27.07.2010.

\section{Akademische Lehre an der Georg-August-Universität Göttingen (Auswahl)}

SoSe 2018: Feldforschung (M.Mus.33; Musikwissenschaftliches Seminar; begleitende Lehrende; hauptverantwortlich Dr. Eva-Maria van Straaten)

WiSe 2016/2017: Rassismus im Alltag. Von den Bedeutungen zur Theorie (SQ.Sowi.13; Sozialwissenschaftliche Fakultät; begleitende Lehrende; hauptverantwortlich PhD. Sascha Kesseler)

SoSe 2009: Kulturwissenschaftliche Literaturwissenschaft (Hauptseminar der Allgemeinen \& vergleichenden Literatur- und Kulturwissenschaft; Lehrauftrag)

SoSe 2008/2009: Postcolonial Women's Writing: Literatur Ozeanien (Hauptseminar der Allgemeinen und vergleichenden Literatur- \& Kulturwissenschaft; Lehrauftrag) 


\section{Erklärung}

Gemäß $\int 12$ der Prüfungsordnung für den Promotionsstudiengang Sozialwissenschaften an der Sozialwissenschaftlichen Fakultät der Georg-August-Universität Göttingen aus dem Jahr 2013 möchte ich folgendes versichern:

1. Die Gelegenheit zum vorliegenden Promotionsvorhaben ist mir nicht kommerziell vermittelt worden. Insbesondere habe ich keine Organisation eingeschaltet, die gegen Entgelt Betreuerinnen und Betreuer für die Anfertigung von Dissertationen sucht oder die mir obliegenden Pflichten hinsichtlich der Prüfungsleistungen für mich ganz oder teilweise erledigt.

2. Ich versichere, dass ich die eingereichte Dissertation mit dem Titel: Lernen und das Andere. Hochschuldidaktische Erkenntnisse zur Anwendung des Konzeptes der Alterität auf Lehr-und Lernprozesse im akademischen Kontext selbstständig und ohne unerlaubte Hilfsmittel verfasst habe; fremde Hilfe habe ich dazu weder unentgeltlich noch entgeltlich entgegengenommen und werde dies auch zukünftig so halten. Anderer als der von mir angegebenen Hilfsmittel und Schriften habe ich mich nicht bedient. Alle wörtlich oder sinngemäß den Schriften anderer Autoren entnommenen Stellen habe ich kenntlich gemacht.

3. Die eingereichte Dissertation habe ich nicht bereits in einem anderen Prüfungsverfahren vorgelegt.

4. Des Weiteren ist mir bekannt, dass Unwahrhaftigkeiten hinsichtlich der vorstehenden Erklärung die Zulassung zur Promotion ausschließen bzw. später zum Verfahrensabbruch oder zur Rücknahme des erlangten Titels berechtigen.

Eingereicht zur Begutachtung am 29.03.2019

Veröffentlicht im Juni 2019

Göttingen, den 24.06.2019

Angelika Thielsch 